University of Louisville

ThinkIR: The University of Louisville's Institutional Repository

Electronic Theses and Dissertations

8-2017

\title{
Fabrication and characterization of a mcro/nanofluidic platform for electroporation.
}

Hanwen Yuan

University of Louisville

Follow this and additional works at: https://ir.library.louisville.edu/etd

Part of the Nanoscience and Nanotechnology Commons

\section{Recommended Citation}

Yuan, Hanwen, "Fabrication and characterization of a mcro/nanofluidic platform for electroporation." (2017). Electronic Theses and Dissertations. Paper 2757.

https://doi.org/10.18297/etd/2757

This Doctoral Dissertation is brought to you for free and open access by ThinkIR: The University of Louisville's Institutional Repository. It has been accepted for inclusion in Electronic Theses and Dissertations by an authorized administrator of ThinkIR: The University of Louisville's Institutional Repository. This title appears here courtesy of the author, who has retained all other copyrights. For more information, please contact thinkir@louisville.edu. 
FABRICATION AND CHARACTERIZATION OF A MICRO/NANOFLUIDIC PLATFORM FOR ELECTROPORATION

\author{
by \\ Hanwen Yuan \\ M.S., South China University of Technology, 2009 \\ China
}

\begin{abstract}
A Dissertation
Submitted to the Faculty of the

J. B. Speed School of Engineering of the University of Louisville

in Partial Fulfillment of the Requirements

for the Degree of

Doctor of Philosophy

in Mechanical Engineering

Department of Mechanical Engineering

University of Louisville

Louisville, Kentucky
\end{abstract}

August 2017 



\section{FABRICATION AND CHARACTERIZATION OF A MICRO/NANOFLUIDIC} PLATFORM FOR ELECTROPORATION

$$
\text { by }
$$

Hanwen Yuan

A Dissertation Approved on

April 25, 2017

by the following Dissertation Committee:

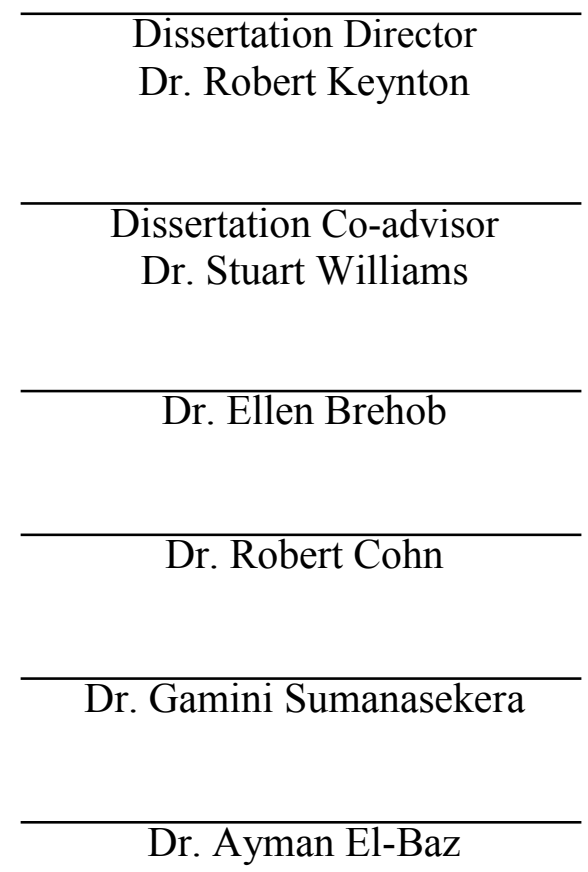




\section{DEDICATION}

This dissertation is dedicated to my parents,

my wife, my son and to all of my family and friends. 


\section{ACKNOWLEDGEMENTS}

I would like to express my deepest gratitude to my advisor, Dr. Robert Keynton for taking me under his wing and for his continuous support, inspiration, and guidance throughout my research career at the University of Louisville. I am deeply grateful for the enthusiasm towards micro/nanofabrication research that Dr. Keynton installed into me and for his encouragement and wisdom in directing that energy towards pushing back the scientific envelope. Next, I would like to thank my co-advisor, Dr. Williams for training me in cell trapping experiments, and for many hours of discussion on all aspects of this project. I would like to acknowledge Dr. Patricia Soucy for patiently educating a mechanical engineering student on the intricacies of cell culture and cell viability assay and for broadening my horizons to the biology field. And I would like to thank Dr. Ayman El-Baz for educating me processing experimental data in a high efficient way. I also want to thank my other three committee members Dr. Robert Cohn, Dr. Gamini Sumanesekera, and Dr. Ellen Brehob for their unconditional assistance and discussion involving scientific, professional, and sometimes personal challenges.

I offer my thanks to other faculty and staff members in the Mechanical Department, the Electrical and Computer Engineering Department and the Bioengineering Department for their support. Specifically, I would like to thanks Drs. Scott Camrbon, Mark Crain and Thomas Roussel for their help on instrumentation and scientific discussion and constructive suggestion and Mr. Douglas Jackson for his help in instrument 
troubleshooting. I am also grateful for the support of Dr. Martin O'Toole, Mrs. Betty Nunn, and Miss. Larissa Pack.

I also want to thank a number of my fellow research scientists and graduate students who were of great support as friends and colleagues throughout my graduate studies: Peng Xu, Li Zhu, Xiaoli Wei, Xinghua Sun, Kurtis James, Ben King, Dakota Waldecker, Monica Moreno Ruano.

All my family members have been very supportive in every aspect of my life. I need to thank my parents Heyang Yuan and Yuxiang Wu for their love and the great education they gave to me. And without their help to take care of my baby, this dissertation would not be possible. I want to thank my sister Liuqun Yuan and my brother Hanwu Yuan for their support. I want to thank my wife Xue Shi for her love and support and thank my son Oliver Yuan bring me all the joy and happiness. 


\title{
ABSTRACT \\ FABRICATION AND CHARACTERIZATION OF A MICRO/NANOFLUIDIC PLATFORM FOR ELECTROPORATION
}

\author{
Hanwen Yuan
}

April 25, 2017

For traditional electroporation devices, there are a number of problems associated with these devices such as insufficient understanding of its theoretical mechanism, low cell viability, inadequate electroporation efficiency, excess voltage applied to generate required electric field due to the large size of these devices and sample contamination. Although newly developed microfluidic electroporation devices have solved most of the above existing problems in traditional bulk electroporation devices, they appear to lack the ability to control the precise dose of biomolecules or genes transfecting into cells and, from a manufacturing perspective, the fabrication methods do not enable repeatable production of such devices on the large scale. Here, we introduce a new, repeatable method for fabricating 3-D Micro/Nanofluidic electroporation platforms and characterize these platforms to demonstrate their ability to electroporate live cells.

Some of the new methods developed in this work include a direct-write fiber technique via three-axis robotic dispensing system, dry film resist photolithography, filmto-film bonding and replica molding to create the desired electroporation platform. A robotic dispensing system was utilized to control the fiber diameter, which was determined 
by the: 1) prescribed dispense time; 2) pressure of the dispensing system valve; 3) rate at which the stage traversed; 4) diameter of the dispensing tip; 5) polymer solution viscosity and surface tension; and, 6) programmed drawing length. Thin dry film photoresist was utilized to replace liquid photoresist in order to achieve high-quality film-to-film bonding after drawing nanofibers onto one substrate containing the thin-film structure. Polydimethylsiloxane (PDMS) was employed as the bulk material to fabricate the target micro/nano electroporation substrate using replica molding and micro/nanofibers etching.

Characterization of the direct-write fiber technique via robotic dispensing system to acquire suspended and complex fibers of the required dimension repeatedly under prescribed conditions were completed. Combining this fiber direct-write method and traditional clean room techniques, a total of 18 micro- to nano-scale electroporation devices (6 for each group of $1 \mu \mathrm{m}, 500 \mathrm{~nm}$, and $300 \mathrm{~nm}$ diameter) were successfully developed and mass produced in two weeks with relatively high repeatability (within $20 \%$ of the design). Finally, metrology and characterization studies were performed on the electroporation platforms to validate the micro/nanochannel's existence and its connectivity to two microchambers. Furthermore, biomolecules and other fluorescent particles were successfully transported through the micro/nanochannel and transferred (via electroporation) into the cells. Preliminary results of electroporation experiment performed on this micro/nanoelectroporation platform illustrated that the duration of the entire electroporation process was significantly shorter than times reported previously by other investigator's nanoelectroporation platforms. 


\section{TABLE OF CONTENTS}

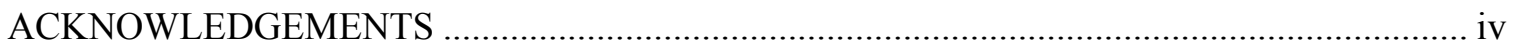

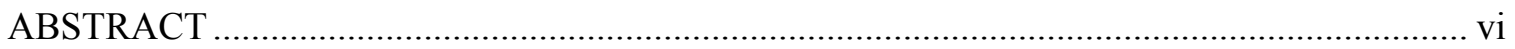

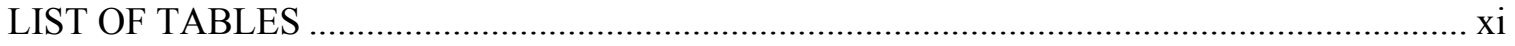

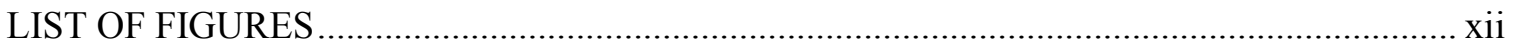

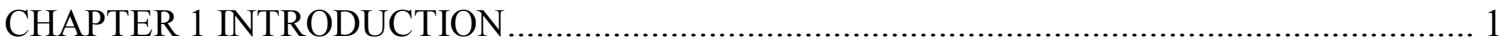

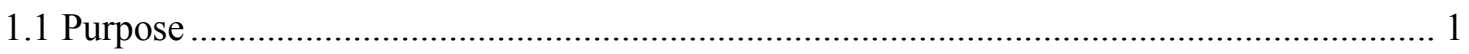

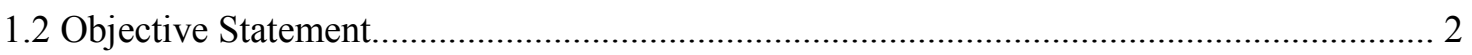

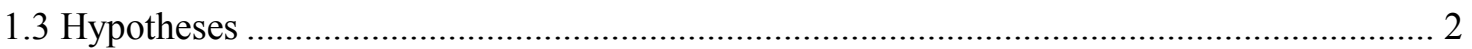

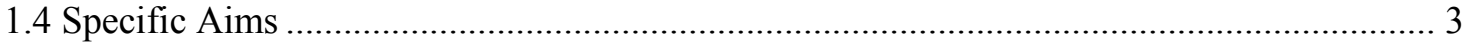

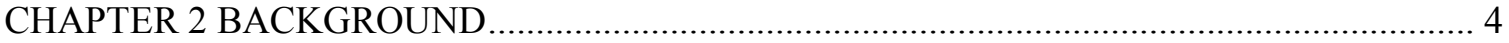

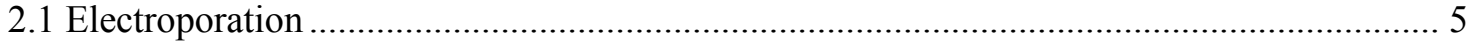

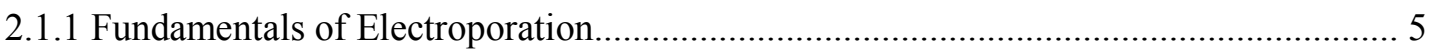

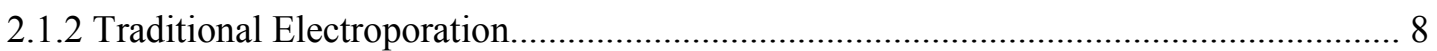

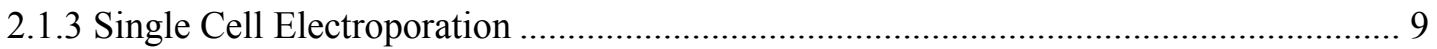

2.2 Overview of Existing Electroporation Platforms …........................................................ 10

2.2.1 Application in Bulk or Micro/Nano Pipette Platforms ............................................... 10

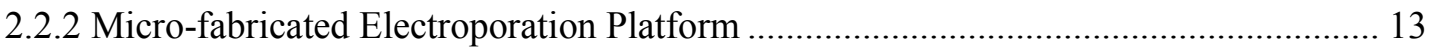

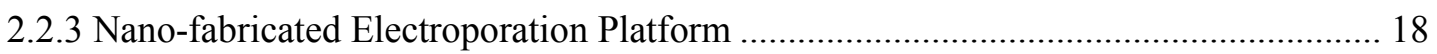

2.2.4 Biomolecules Transport Mechanism in Micro/Nano-fabricated Devices ..................... 21

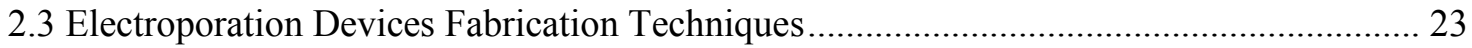

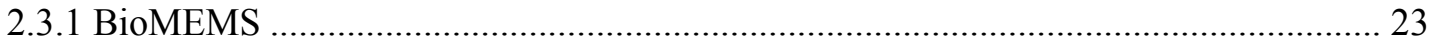

2.3.2 Micro/Nanofabricated Electroporation Platform Fabrication....................................... 24

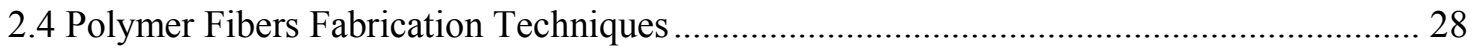

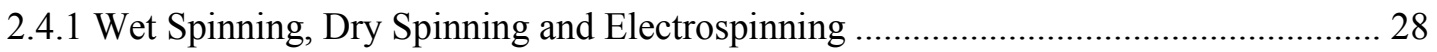

2.4.2 AFM Tip and Hand-brush Based Fiber Fabrication Methods ....................................... 30

2.4.3 Principle of Polymer Solution Capillary Thinning and Breakup.................................. 34

2.4.4 Dimensionless Parameters of Polymer Solution Filament Formation.......................... 36

2.4.5 Direct Write Fibers via Micromilling Machine …........................................................ 39

2.4.6 Direct Write Fibers via Robotic Dispensing System ................................................ 41

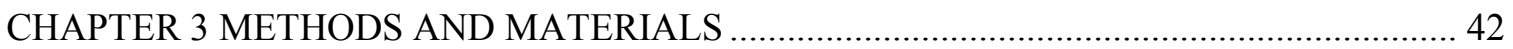

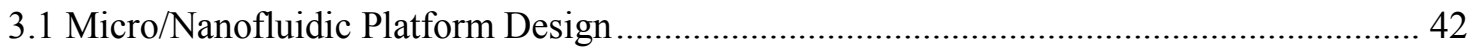




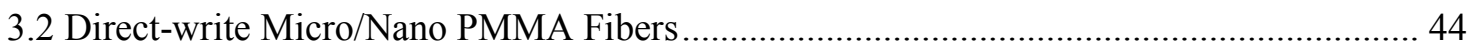

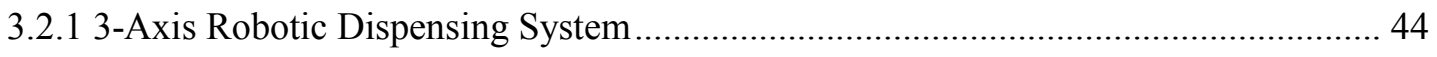

3.2.2 Overview of the 3-axis Robotic Dispensing System Direct-write Process ................. 47

3.2.3 Direct-write Procedure for Complex Suspended Fiber Structures ............................... 50

3.2.4 Characterization of Direct-write Process..................................................................... 53

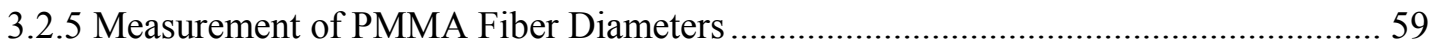

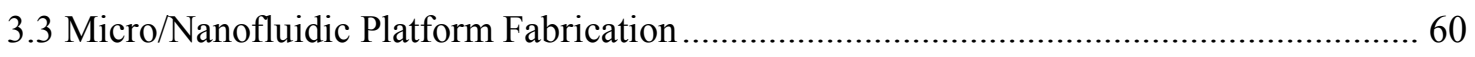

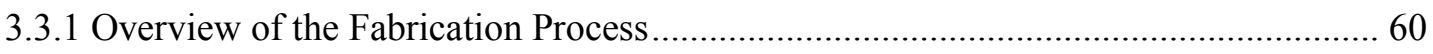

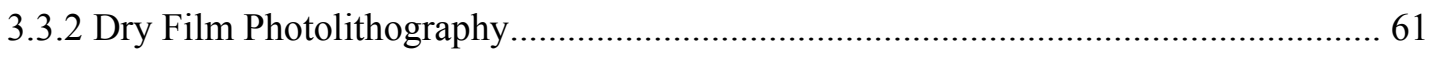

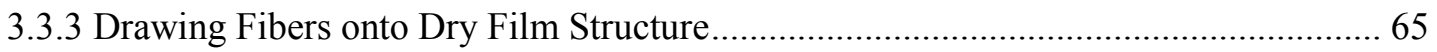

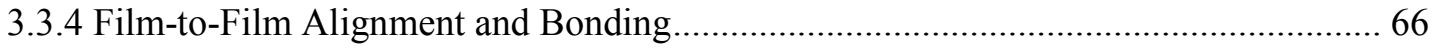

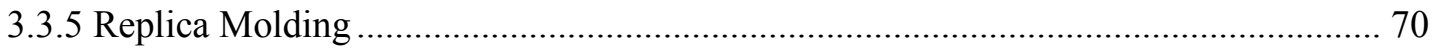

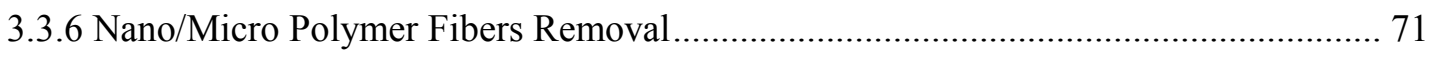

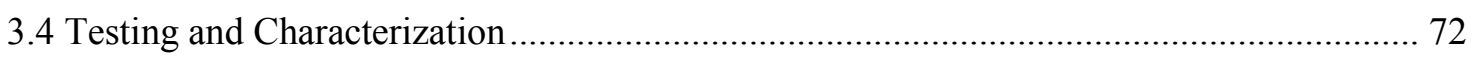

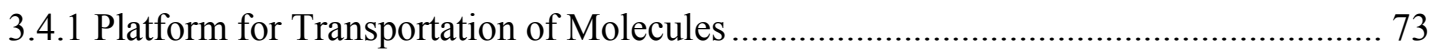

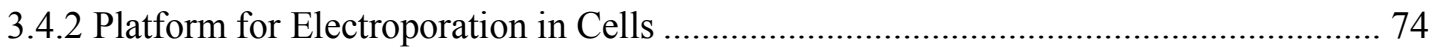

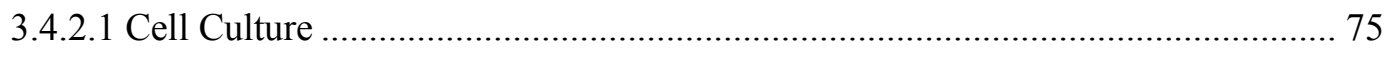

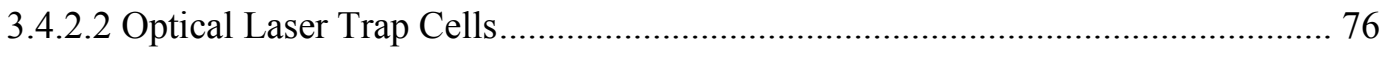

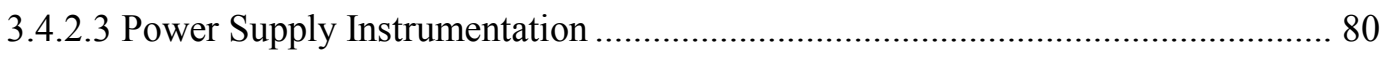

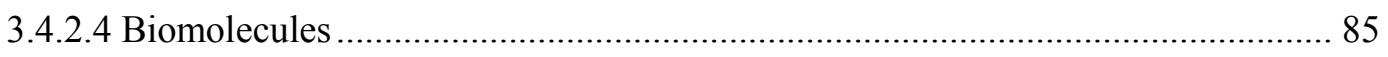

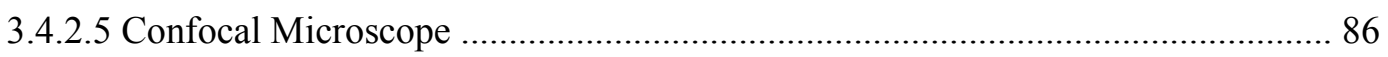

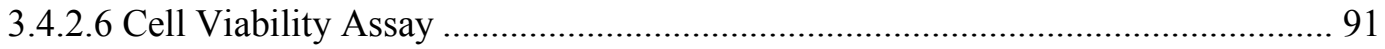

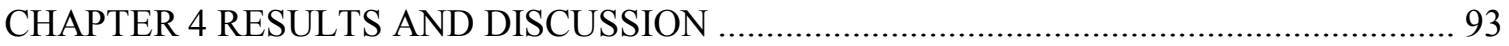

4.1 Implementation of a 3-axis Robotic System to Direct-write 3-D Micron/Sub-micron Fiber

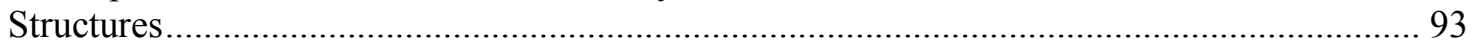

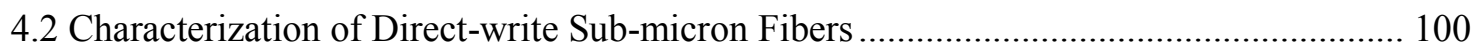

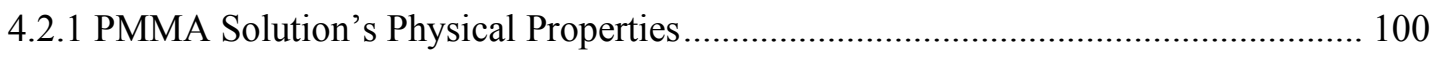

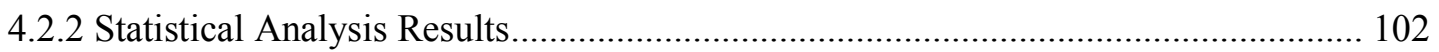

4.2.3 Empirical Prediction Equation Generation ........................................................... 111

4.2.4 Minimum Diameter of PMMA Fibers.................................................................... 111

4.2.5 Validation of Empirical Equation........................................................................ 113

4.3 Fabrication of 3-D Micro/Nano Fluidic Electroporation Devices ................................... 116

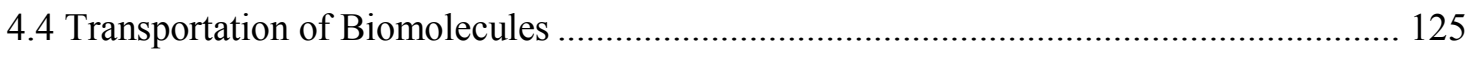

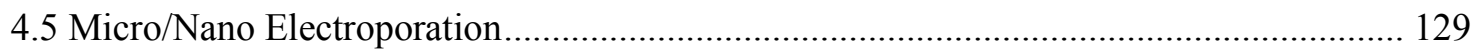

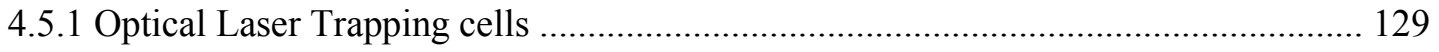

4.5.2 Micro/Nano Electroporation Preliminary Results ..................................................... 131 


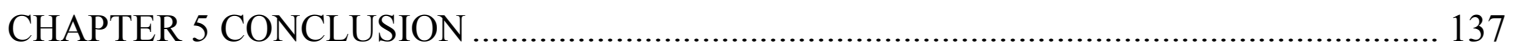

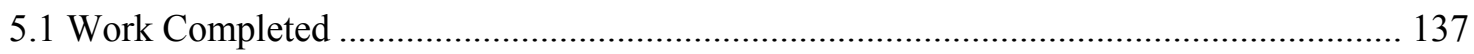

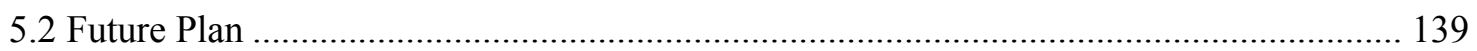

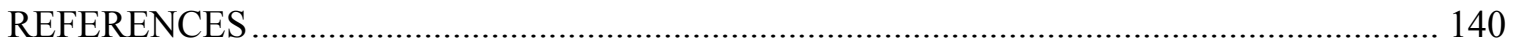

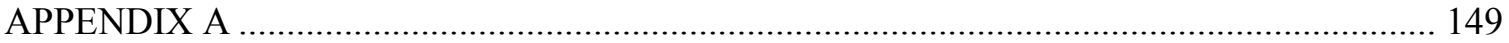

APPENDIX B

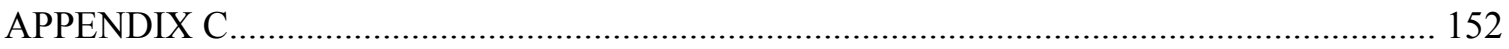

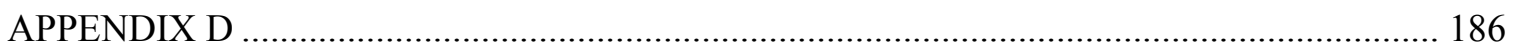

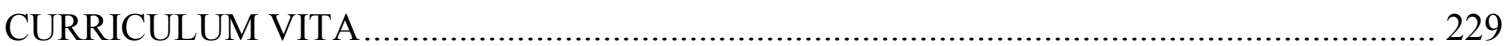




\section{LIST OF TABLES}

Table 3. 1 Direct-write process operating parameters used in the characterization ...................... 54

Table 3. 2 Critical direct-write process parameters identified in the characterization .................. 55

Table 3. 3 Optimized parameters for the DFRs photolithography processes on ......................... 63

Table 3. 4 Optimized parameters for the DFRs photolithography processes ............................... 64

Table 3. 5 Electrical connections for the printed board and DAQ channels .............................. 82

Table 4. 1 Physical properties of PMMA solutions at different............................... 101

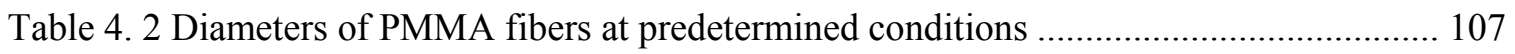

Table 4. 3 Optimized diameters of suspended PMMA fibers onto hollow Substrate ................. 108

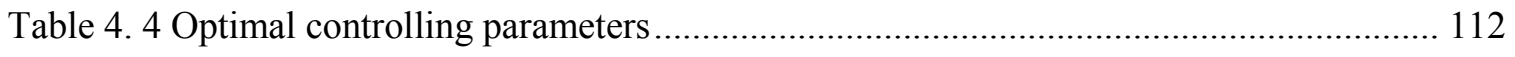

Table 4. 5 Performance data of fibers at optimal condition with 15 psi pressure ....................... 113

Table 4. 6 Performance data of fibers at optimum condition at 3 psi pressure .......................... 115

Table 4. 7 Comparison between the design and actual dimensions achieved f......................... 118

Table 4. 8 Comparison of fiber diameters to actual channel diameters for .............................. 118

Table 4. 9 Direct-Write Process Optimized Parameters for Three Different ............................. 119

Table 4. 10 Current flow and energy passivation across cell membrane for different............... 136 


\section{LIST OF FIGURES}

Figure 2. 1 Scheme of induction of transmembrane potential ................................................ 6

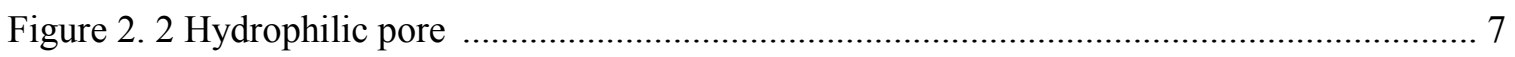

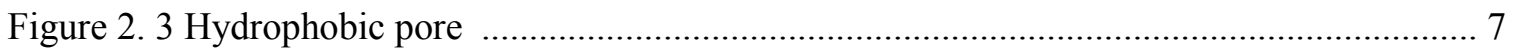

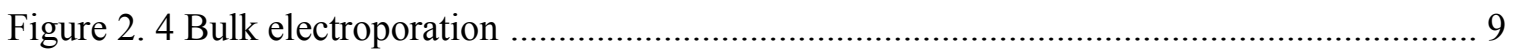

Figure 2. 5 Schematic eesign of bulk electroporation platforms ............................................ 11

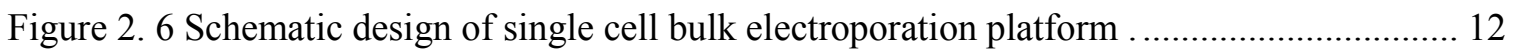

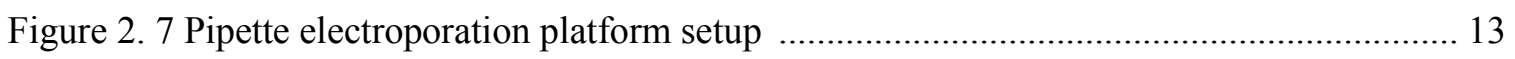

Figure 2. 8 Schematic design of different microfluidic electroporation platforms …................. 15

Figure 2. 9 Images and schematics of an array of narrow lateral channels ............................... 16

Figure 2. 10 Schematic diagram of the membrane sandwich electroporation technique .............. 17

Figure 2. 11 Schematic diagram of localized SCEP platform ................................................ 18

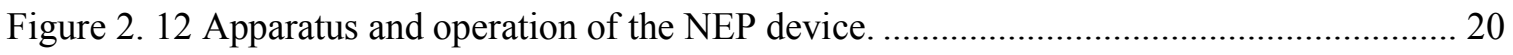

Figure 2. 13 Theoretical analysis of nanofluidic electroporation system .................................. 20

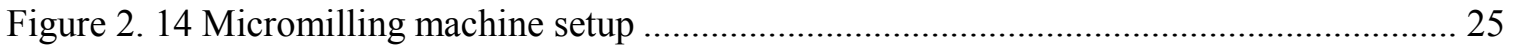

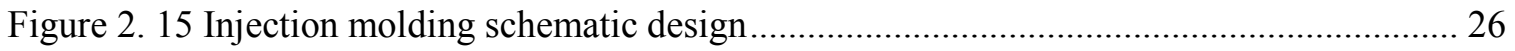

Figure 2. 16 Schematic representation of microfluidic channel fabrication............................... 27

Figure 2. 17 Schematic of a typical electrospinning apparatus .............................................. 29

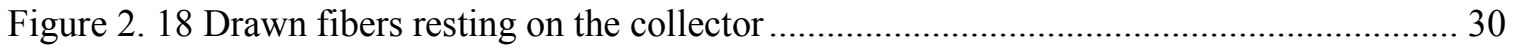

Figure 2. 19 AFM tip drawing suspended fiber structures ..................................................... 31

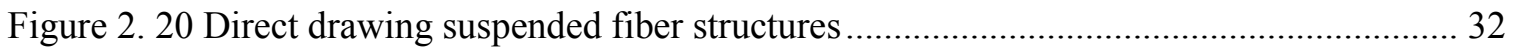

Figure 2. 21 SEM image of suspended and oriented fiber structures ........................................ 33

Figure 2. 22 Fibers drawn between a sharp tip Array and a layers of polymer........................... 33 
Figure 2. 23 Schematic illustrating the direct-write drawing of fibers ...................................... 40

Figure 2. 24 Micro-Milling Machine and its constituent parts ................................................. 40

Figure 3. 1 Schematic diagram of the design of the Micro/Nanofluidic ........................43

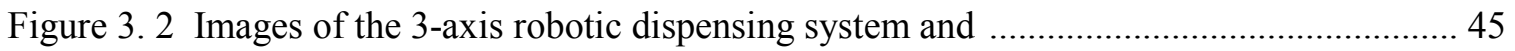

Figure 3. 3 Image of the display panel in the JR-C Points software ......................................... 46

Figure 3. 4 Image of the display panel in the JR-C Points software for defining ....................... 46

Figure 3. 5 Control panel display on the 3-axis robotic system with the dispense time............... 46

Figure 3. 6 Image of a typical syringe needle tip (25 Gauge needle shown here)...................... 47

Figure 3. 7 Schematic of the direct-write process via the Robotic Dispensing System.............. 49

Figure 3.8 a.) Example of the design for the "Quad" web structure created in............................ 51

Figure 3.9 a) Example of the design for the "Symmetric" web structure created ....................... 51

Figure 3. 10 a) Example of the design of the "Dual Chevron" web structure created .................. 52

Figure 3. 11 Illustration of how the actual coordinates were determined for .............................. 52

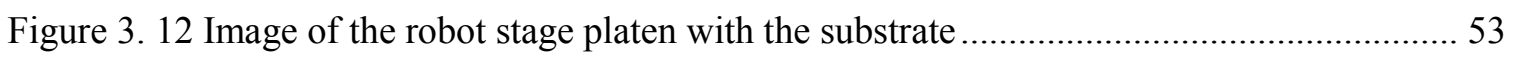

Figure 3. 13 Schematic of the process flow for fabricating the electroporation devices............... 61

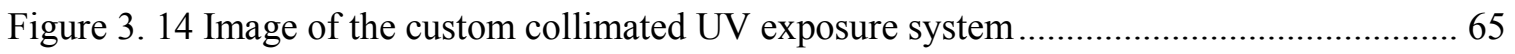

Figure 3. 15 Image of the custom-made film-to-film alignment and bonding system ................. 68

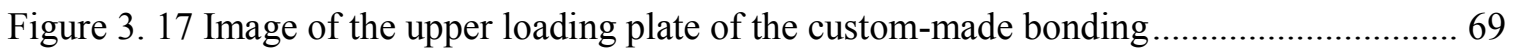

Figure 3. 18 Images of the substrate mold covered with PDMS (right) in the ............................. 71

Figure 3. 19 PDMS Substrate in an Ultrasonic Acetone Bath..................................................... 72

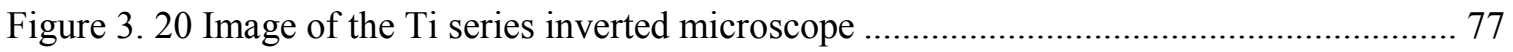

Figure 3. 21 Image of the Nikon $60 \times$ objective lens used in the laser trapping experiments........ 78

Figure 3. 22 Image of the laser diode and temperature controllers for the laser trapping system. 78

Figure 3. 23 Circuit diagram of the custom-made power supply ........................................... 81

Figure 3. 24 Circuit board layout of the custom-made power supply ........................................ 82 
Figure 3. 25 Full schematic of the USB-6009[131]

Figure 3. 26 Block diagram of the monitoring the real-time pulse algorithm 85

Figure 3. 27 Block diagram of the moving average filter algorithm.... 85

Figure 3. 28 Image of the confocal microscope and scan head (Nikon Eclipse Ti)..... 86

Figure 3.29 Image of the LED light source for the confocal microscope (Nikon Eclipse Ti) ..... 87

Figure 3. 30 Image of the display panel in the confocal microscope software (Ti Pad) .............. 88

Figure 3. 31 Image of the display panel for the confocal microscope software (LUTs) ............... 89

Figure 3. 32 Image of the display panel for the confocal microscope software ........................... 90

Figure 4. 1 3D suspended structures fabricated from a 24\% PMMA polymer solution............... 94

Figure 4. 2 3D suspended structures fabricated from a 24\% PMMA polymer solution: a........... 95

Figure 4. 3 3D suspended structures fabricated from a 24\% PMMA polymer solution: a.) ......... 95

Figure 4. 4 SEM images of suspended, branched fiber structures.............................................. 96

Figure 4. 5 SEM image illustrating the sequential point-to-point order of fabrication of............. 97

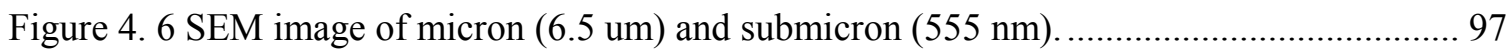

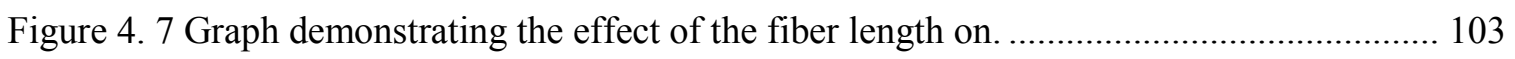

Figure 4. 8 Graph demonstrating the effect of fiber length on drawn fiber............................... 103

Figure 4. 9 Graph demonstrating the effect of fiber length on drawn f................................... 104

Figure 4. 10 Graph demonstrating the effect of fiber length on drawn fiber diameter ............... 104

Figure 4. 11 An optical image of PMMA fibers drawn on glass substrate with ........................ 106

Figure 4. 12 PMMA fibers drawn on the hollow substrate with different lengths ..................... 107

Figure 4. 13 Optical (upper left) and SEM (the three insets) images of PMMA........................ 108

Figure 4. 14 SEM images of PMMA fibers with specific diameter at predetermined ................ 110

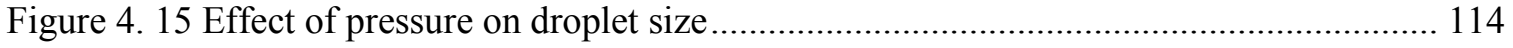

Figure 4. 16 Number of prediction errors less than $15 \%$ at different pressure .......................... 116

Figure 4. 17 SEM image of the DFRs mold on a glass substrate with ...................................... 120 
Figure 4. 18 SEM image of the dry film resist mold on a glass substrate with a microscale ...... 121

Figure 4. 19 SEM image of the dry film resist mold on a glass substrate with ......................... 122

Figure 4. 20 SEM image of the dry film resist mold on a glass substrate ................................ 123

Figure 4. 21 SEM image of two layer dry film resist mold on a glass ..................................... 123

Figure 4. 22 a) Optical image of the micron channel $(\sim 973 \mathrm{~nm})$ embedded within t................. 124

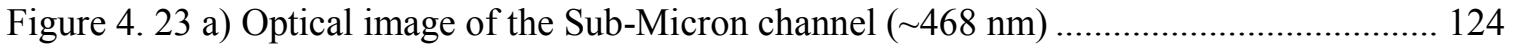

Figure 4. 24 a) Optical image of the Nanochannel ( $292 \mathrm{~nm})$ embedded................................. 125

Figure 4. 25 Confocal microscope image of PI dye transporting through embedded ................. 126

Figure 4. 26 Confocal microscope image of PI dye transporting through embedded. ................ 127

Figure 4. 27 Confocal microscope image of PI dye transporting through................................. 127

Figure 4. 28 Confocal microscope snapshot image of PI dye travelling through....................... 128

Figure 4. 29 Optical image of single cell trapped (wavelength is $980 \mathrm{~nm}$ ) ….......................... 130

Figure 4. 30 Optical image of four cells laser trapped (wavelength is $980 \mathrm{~nm}$ ) ........................ 130

Figure 4. 31 Optical images of single cell trapping process (wavelength is $980 \mathrm{~nm}$ )............... 131

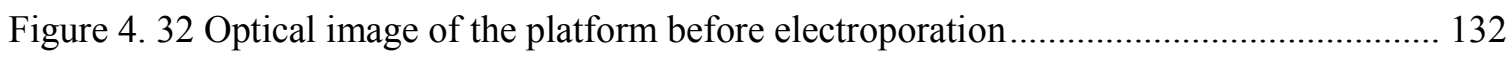

Figure 4. 33 Optical image of the platform (dimension $476 \mathrm{~nm}$ ) After Electroporation.............. 132

Figure 4. 34 Sequential images of single cell electroporation process for a $476 \mathrm{~nm}$ channel ..... 133

Figure 4. 35 Optical image of the platform (dimension 1.41) before and after electroporation.. 133 


\section{CHAPTER 1 INTRODUCTION}

\subsection{Purpose}

Electroporation is a molecular biology technique that encompasses applying an electrical field to cells to create a pore on the cell membrane in order to transfect biomolecules, drugs, DNA, and genes into the cells. Cell electroporation has attracted many researchers' attention due to its broad application such as cell analysis and transfection or pasteurization. Specifically, these applications can be defined as two main groups. The first group mainly investigates how to release intracellular nucleic acids, proteins, and other metabolites in order to study the effects on the cell. The second group is trying to deliver exogenous reagents including drugs, genes, and biomolecules into cells for therapeutic applications. In order to fulfill the promising application of cell electroporation, numerous researchers have developed different types of electroporation platforms for their specific purposes. Cell electroporation requires a significant electric field gradient, which "stresses" the cell. When applied over the entire cell or a large area of the cell, the electric field will generate a plethora of pores in the cell membrane; thereby, allowing a mass uptake of not only the targeted molecules, but non-desired compounds, chemicals and other biomolecules as well. Thus, it is desired to minimize not only the number of pores opened on the membrane, but the pore size as well. Most electroporation devices are limited due to low cell viability rate, low transfection efficiency, higher sample contamination, and larger Joule heating effect [1]. Although recently developed microfluidic electroporation platforms overcome most of the existing problems, they lack 
the ability to precisely control the dose of biomolecules transfecting into cells, or enable repeatable production of such devices on the large scale. Based on the current issues in this field, our main task is to design a 3-D micro/nanofluidic electroporation platform and develop a process to mass fabricate these devices on a large scale. These platforms will be utilized in nanoscale cellular applications to precisely control the dose of biomolecules delivered to the cells.

\subsection{Objective Statement}

The objective of this study is to design and develop a micro/nano scale fluidic device that will ultimately be applied to create a nanopore in a cell's membrane in order to transfer biomolecules into cells. Creation of this micro/nanoscale fluidic device requires the use and characterization of a robotic dispensing system to produce a specific range of fiber diameters and dispense them onto precise locations on the platform and to mass produce different fiber dimensions to create micro/sub-micro/nano-electroporation devices for cellular electroporation.

\subsection{Hypotheses}

The hypotheses of this work are:

1. A newly developed direct-write process using a commercially-available 3-D axis robotic system improves process efficiency (fast with high yield), structure complexity (suspended fibers with high spatial control) and fiber dimension (precise control of fiber dimensions) compared to the previously developed method using an ultra-high precision micro-milling machine. 
2. The direct-write process will repeatedly draw PMMA fibers within $20 \%$ of the design fiber diameter $(\sim 300 \mathrm{~nm}, \sim 500 \mathrm{~nm}, \sim 1000 \mathrm{~nm})$.

3. Electroporation platforms will be repeatedly manufactured with micro-/sub-micro/nano-channel diameters and micro-chamber gap distances and depth within 20\% of the design values.

4. At least $80 \%$ of the cells will be viable after electroporation and the electroporation process will be achieved in a much shorter time frame than previously reported results within micro-/sub-micro-/nano-electroporation devices.

\subsection{Specific Aims}

Specific Aim 1: Characterize the direct-write capabilities of the 3-D axis robotic system and compare its performance to previously published results from an ultra-high-precision micro-milling machine.

Specific Aim 2: Characterize the robotic dispensing system to prioritize the key parameters of the direct write process responsible for fiber formation via statistically adjusting the experimental parameters and mathematically model the direct-write process to identify the corresponding controlling parameters. Correlate the key parameters of the direct-write process to the physical properties for controlling fiber diameter including fiber length, feed rates, needle tip size, polymer solutions weight concentration, and valve dispensing time. Specific Aim 3: Design and develop electroporation platforms using traditional microfabrication processes in conjunction with the 3-D axis robotic system.

Specific Aim 4: Transfer PI dye into HL60 cells located in the micro-/sub-micro-/nanoelectroporation devices under a high electric field. 


\section{CHAPTER 2 BACKGROUND}

Cells are the basic structural, functional, and biological building blocks of all known living things. Their main function is to take in nutrients and convert them into energy to support daily activities. The cell is wrapped by a cell plasma membrane, which is composed of a double layer of lipids in order to separate and protect the cell from the surrounding environment. Inside this plasma membrane, there are a lot of different types of proteins acting as channels or pumps to create a pathway to transport simple molecules, while for other types of biomolecules, this plasma membrane acts as an impenetrable barrier. Cell transport can be divided into two categories: 1) passive transport; and, 2) active transport. Passive transport requires no energy from the cell to move chemicals and other molecules across the cell membrane such as the diffusion of oxygen, carbon dioxide, and osmosis water, diffusion of water across a semi-permeable cell membrane is called osmosis. Meanwhile, active transport needs energy to transport large molecules and, such as sodium, potassium, glucose and amino acids. While these transport mechanisms are effective in moving ions and molecules naturally across cell membranes, it is difficult to take advantage of these natural processes to controllably transfect desired molecules across the cell membrane. Thus, investigators discovered a means by which to apply an electric field across a cell membrane to create pores in the cell membrane [2]. This chapter will explore the field of electroporation, define the use and application of this technique and describe current methods for fabricating electroporation platforms. 


\subsection{Electroporation}

Electroporation is a molecular biology technique that encompasses applying an electrical field to cells to create a pore on the cell membrane in order to transfect biomolecules, drugs, DNA, and genes into and out of the cells. Cell membrane electroporation technique has been developed for the purpose of being a valuable tool for cell analysis, transfection, and pasteurization. Applying high electric field strengths by placing electrodes in the vicinity of cells can create pores in cell membranes. Electroporation is a dynamic process since it depends on both the magnitude and duration of the applied electrical field at each point on the cell membrane. It is generally accepted that a transmembrane voltage threshold ranges from $0.2 \mathrm{~V}$ to $1 \mathrm{~V}$, where a transmembrane potential is defined as the electric potential difference across the two sides of the cell membrane. If the membrane potential is located in this threshold range, a reversible (temporary) pore will be created. Meanwhile, strengthening the electric pulse increases the size and number of the created pores [3]. But, if the membrane voltage is greater than $1 \mathrm{~V}$, electroporation at these voltages adversely affect cell viability by creating a permanent (irreversible) pore.

\subsubsection{Fundamentals of Electroporation}

Theoretical study of electroporation can be categorized into three groups [3]. The first group focuses on different parameters such as pulse strength and duration, ionic concentration, and field strength impact cell permeabilization [4-6]. The second group pays attention to optimizing and controlling the electric pulse and duration during cell 
electroporation $[7,8]$. The third group mainly studies the transfection of biomolecules into cells during electroporation [9-11].

The cell membrane plays a significant role in explaining the relationship between the external electric field and the transmembrane potential (TMP, Figure 2. 1). A simple equation was given by [12] as:

$$
\Delta \Phi=1.5 r E \cos \theta
$$

where $\Delta \Phi$ is the TMP, $r$ is the cell radius, $E$ is the external electric field, $\theta$ is the angle between the site on the cell membrane and the direction of $E$, where $\Delta \Phi$ is measured and the direction of $E$. Electroporation is achieved when $\Delta \Phi$ imposed on the resting transmembrane is greater than threshold potential.

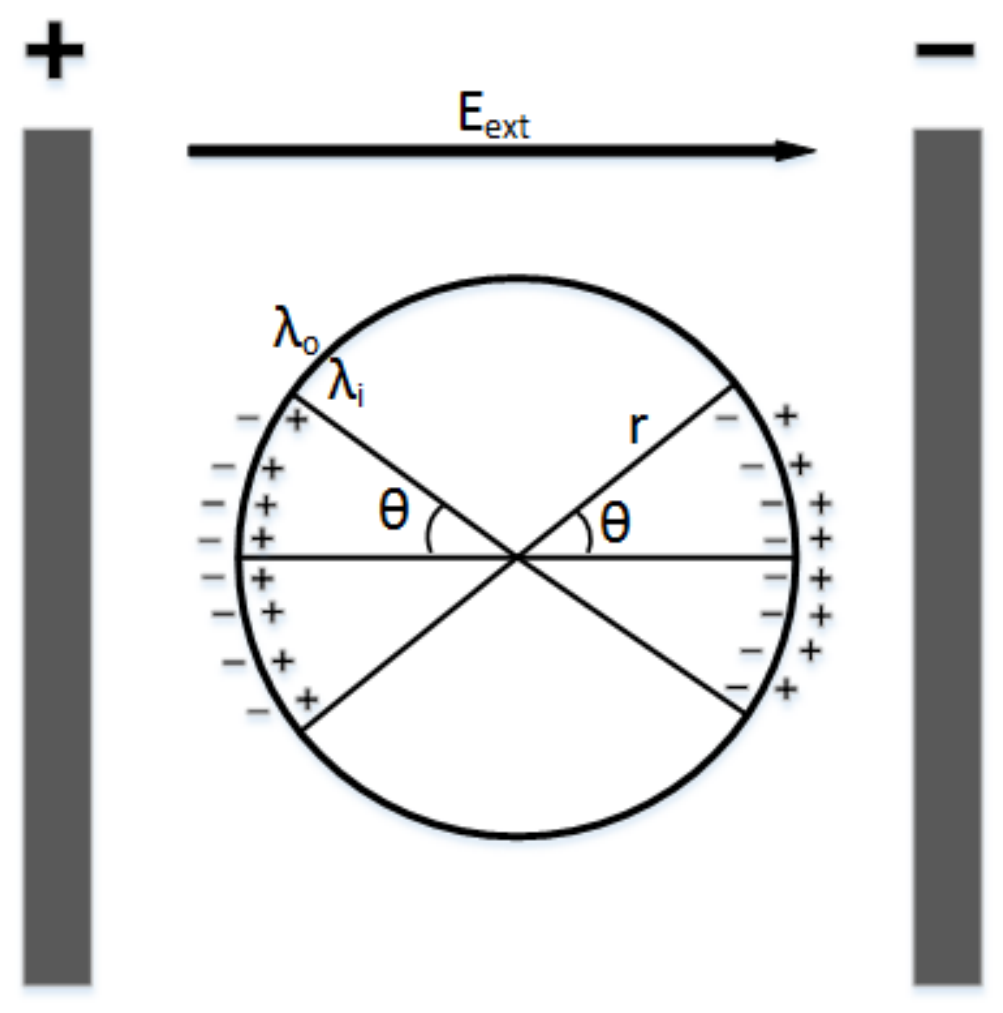

Figure 2. 1 Scheme of induction of transmembrane potential [13] 
A rearrangement of the molecular structure of the cell membrane takes place when the applied TMP arrives at the range of $0.2 \mathrm{~V}$ to $1 \mathrm{~V}$, and the rearrangement will lead to pore formation on the cell membrane. The created pores can be categorized into two different types: hydrophilic pores and hydrophobic pores. Hydrophilic pores have their wall lined with the water-attracting heads of lipid molecules (Figure 2. 2), while hydrophobic pores are gaps in the lipid bilayer of the membrane that are formed by thermal fluctuation (Figure 2. 3) [3]. The dynamic process of pore formation for a cell membrane can be depicted as three steps: pore formation, pore expansion, and pore resealing. A pore is created gradually. A pore will expand if an external constant electric field is applied on the cells. Once the external electric field closes, the pores start to reseal and ultimately disappear completely [13]. However, if the TMP far exceeds threshold, the cell membrane will breakdown since the cell membrane structure will be disturbed significantly, which results in a significant increase in the electrical conductivity and pore formation in the cell membrane permeability to biomolecules, ions or even macromolecules [3].

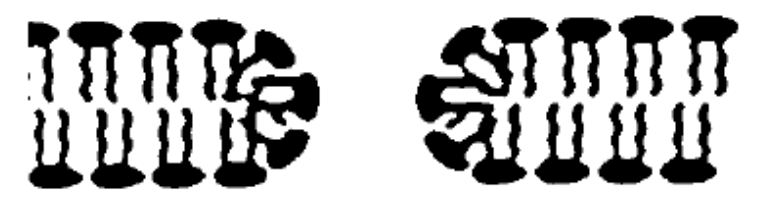

Figure 2. 2 Hydrophilic pore [14]

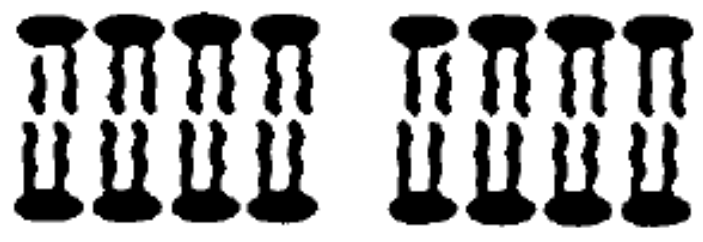

Figure 2. 3 Hydrophobic pore [14] 


\subsubsection{Traditional Electroporation}

Electroporation has been widely applied in the delivery of drugs, DNA, and genes into cells and tissues. In the 1960s and 1970s, numerical and experimental studies were performed on bilayer membranes by Neumann et al. [2, 15], which lead to the first electrophoresis device, using a short-circuiting approach, for transferring genes into murine cells in the 1980s After that, researchers developed more advanced electroporation platforms to improve cell transfection efficiency and lower cell mortality rate. However, the cell transfection efficiency and cell viability rate are still insufficient to meet the increasing demand for developing new drugs, curing diseases, and re-growing organs using the electroporation technique.

with a constant distance on the two opposing sides of a large number of cells. In order to effectively electroporate a large number of cells, a short and high-voltage pulse was required to induce a sufficient transmembrane potential (TMP) to create pores in the cell membranes to pass molecules in the surrounding medium [16]. The proper pulse strength range for reversible electroporation of common mammalian cells varied between $50 \mathrm{~V} / \mathrm{cm}$ to $1000 \mathrm{~V} / \mathrm{cm}$, which depended on the medium, electroporation system, electrical properties, and sizes of the cells [17]. On the one hand, an increase in pulse magnitude and duration time was found to be beneficial for uptake of a large quantity of biomolecules. On the other hand, it resulted in low cell viability caused by Joule heating and irreversible cell membrane recovery after pore creation [18-20]. Furthermore, multiple pores were created on the cell membrane due to the applied electric field (Figure 2. 4), which probably lead to the loss of some significant plasmids in the cells when external biomolecules were transferred into the cells through these pores. As a result, the increase in the number of 
pores led to undesired stress on the cells due to the mass influx of additional external biomolecules, which lower the cell viability as well as transfection efficiency.
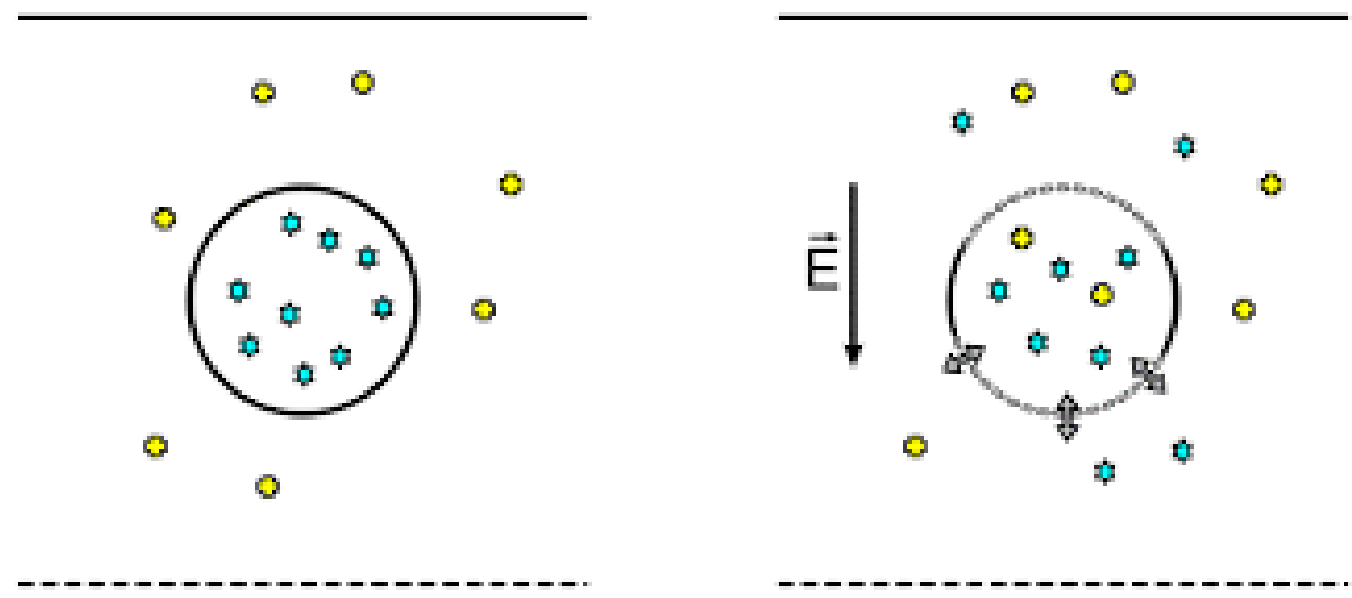

Figure 2. 4 Bulk electroporation [21]

\subsubsection{Single Cell Electroporation}

Compared to bulk electroporation systems, a single cell electroporation system has the advantage of acquiring sufficiently high TMP generated by low applied electric fields, manipulating cells easily, and monitoring the transfection process in real-time. Single cell electroporation has demonstrated promise as a technique for further study of cellular biology and disease processes at the cellular level. However, most past and current research has been based on a cell's size, shape, and orientation, which also affect its electroporation and transfection [22]. The importance of determining the real relationship between the intrinsic and extrinsic properties of cells and electroporation has been recognized, but not fully realized. Most single cell electroporation platforms separated the cell and the biomolecules to be transferred into the cells into two chambers, which greatly reduced the cell's stress by generating fewer pores in the cell membrane. In addition, reducing the single cell's stress improves cell transfection efficiency and cell viability rate significantly 
due to lowering the applied electrical field, shortening the pulse applied on the cell in single cell electroporation platforms.

\subsection{Overview of Existing Electroporation Platforms}

In the beginning, square function pulse generators had been applied to construct electroporation systems in order to modify the amplitude and pulse length independently [23]. In the early stages, artificial planar bilayer membranes had been used to experimentally study the breakdown of bilayer membranes and biomolecules uptake in cells [14, 24-31]. Subsequently, bulk electroporation systems were developed, but these systems yielded low cell viability and transfection efficiency due to slow membrane recovery, excessive swelling, or Joule heating because of the application of high pulse amplitudes and long duration times [17, 32-34]. Later, more advanced systems, including electrolyte-filled capillaries, micropipettes, microelectrodes surrounding cells, and micro/nanofabricated devices had been utilized in cell electroporation [22]. In particular, Lundqvist [35] designed one of the first single-cell electroporation macro-scale devices to study single cell electroporation by using two carbon fiber microelectrodes. Using two carbon fiber electrodes and demonstrated that a single cell can be electroporated with only 1 volt-millisecond square wave pulses by shortening the distance between electrodes with a tiny 2-5 microns and cell surface. In the following, some different types of electroporation devices will be introduced in detail.

\subsubsection{Application in Bulk or Micro/Nano Pipette Platforms}

In bulk electroporation platforms, a large amount of cells is placed between two large parallel electrodes to receive homogenous electric field (Figure 2. 5) [16]. Figure 2- 
5 a) shows the electrode distance varying from top to bottom in the cuvette, which leads to different electric fields applying onto the cells at different locations, while in Figure 2-5 b), cells in the cuvette obtain the same level of electric field strength due to the same electrode distance shown in the right side.

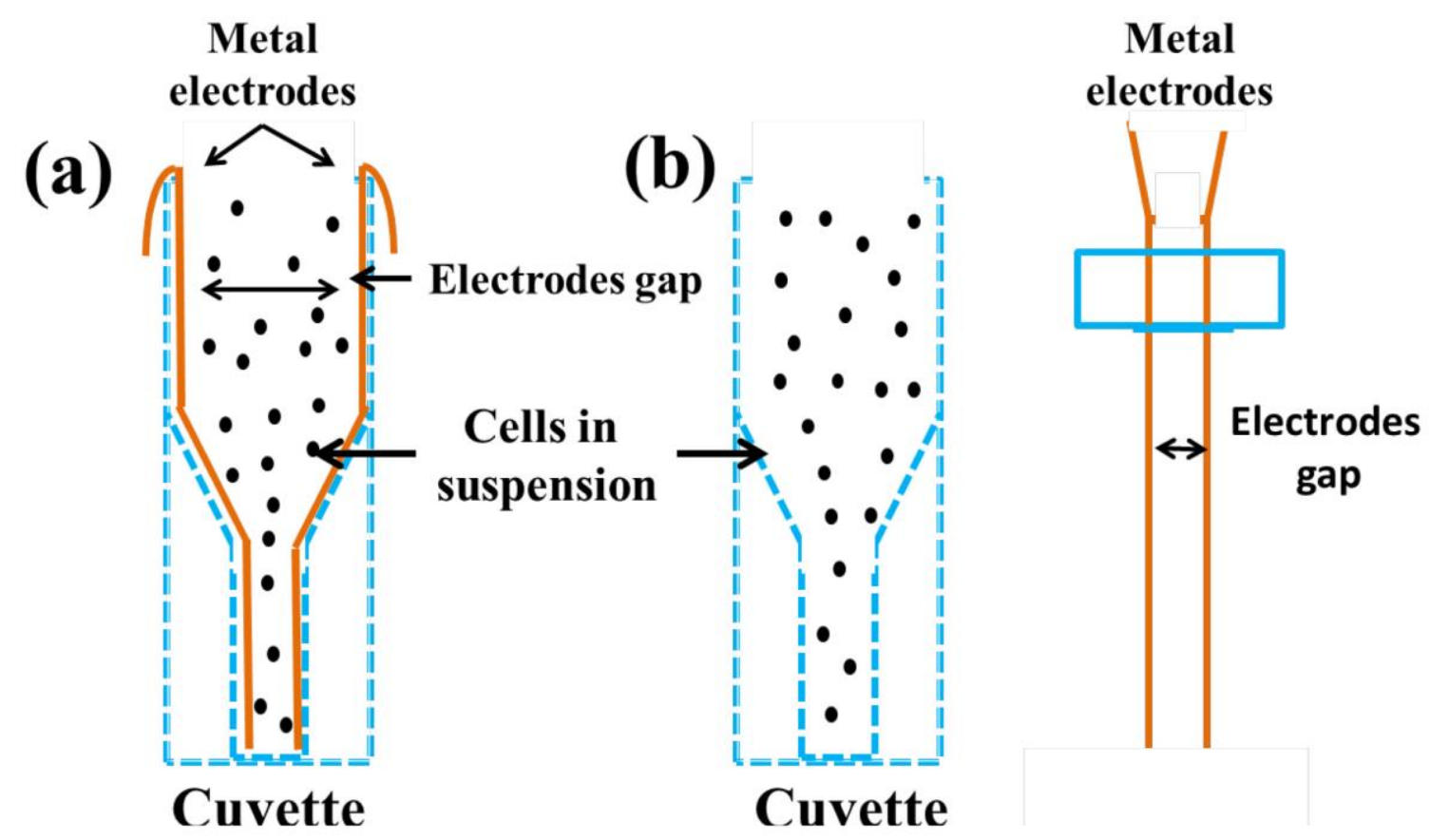

Figure 2. 5 Schematic eesign of bulk electroporation platforms [16].

In order to compare bulk electroporation results with micro- or nanofabricated platform advantages, a single cell bulk electroporation platform was fabricated using a sheet of poly (methyl methacrylate) (PMMA) (Figure 2. 6) [36]. This platform consists of a sheet of PMMA machined by a micromilling machine, an adhesive backed copper film soldered to electrical leads connecting to the power supply, and a glass cover slip sealed to the PMMA. 


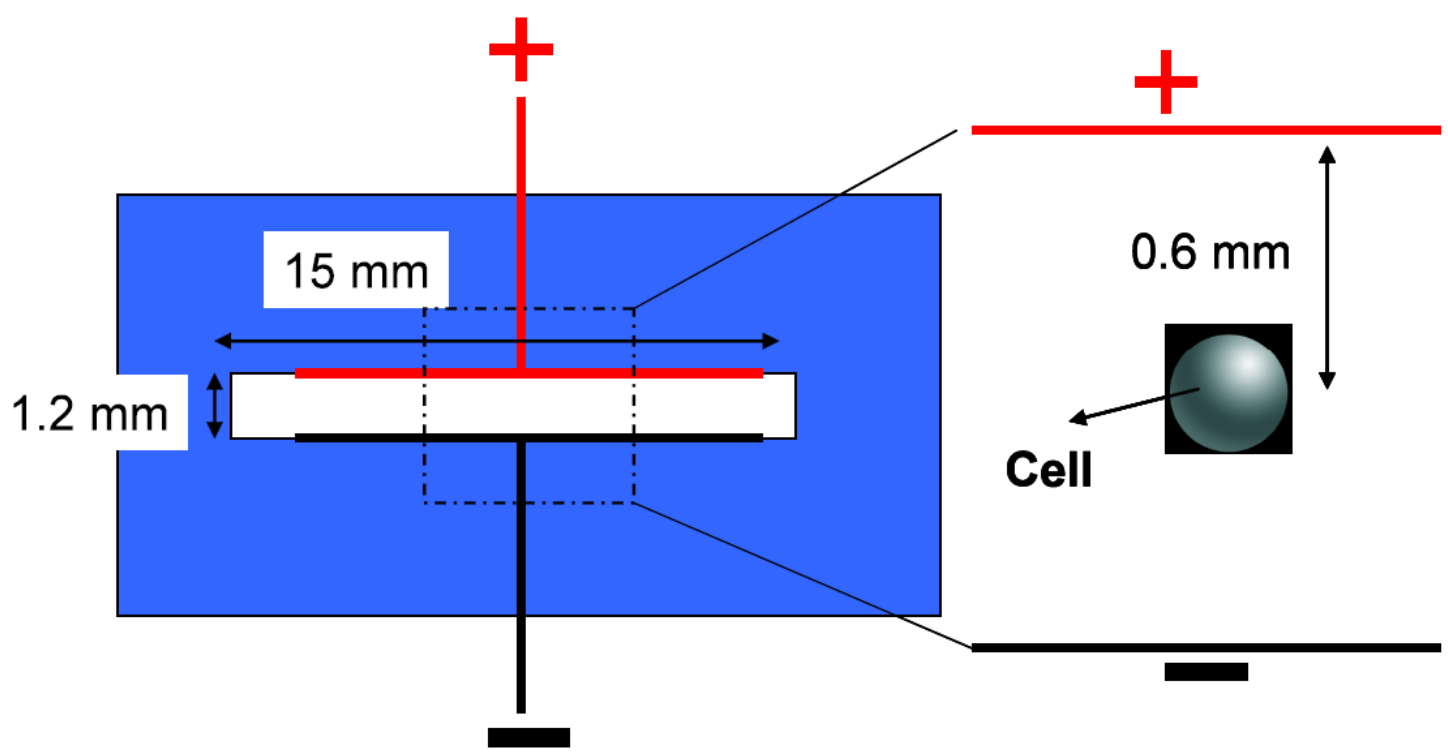

Figure 2. 6 Schematic design of single cell bulk electroporation platform [36].

As we discussed before, to overcome existing issues caused by bulk electroporation platforms, researchers had been developing single cell electroporation (SCEP) platforms including micropipette electroporation devices to apply much smaller electric fields onto a single cell. In these micropipette electroporation platforms, the electric field was applied with a micropipette filled with solute, which was made of filament fused glass coated with a layer of electrode (Figure 2. 7) [37]. The micropipette tip diameter approached the size of a single cell $(\sim 0.5-2 \mu \mathrm{m})$ as close as possible to acquire an adequate transmembrane potential using a low electric field to create a pore on rupture the cell membrane. In this setup, transfection efficiency of $50 \sim 80 \%$ was achieved and demonstrated by real-time visualization using two-photon microscopy during the electroporation process. 


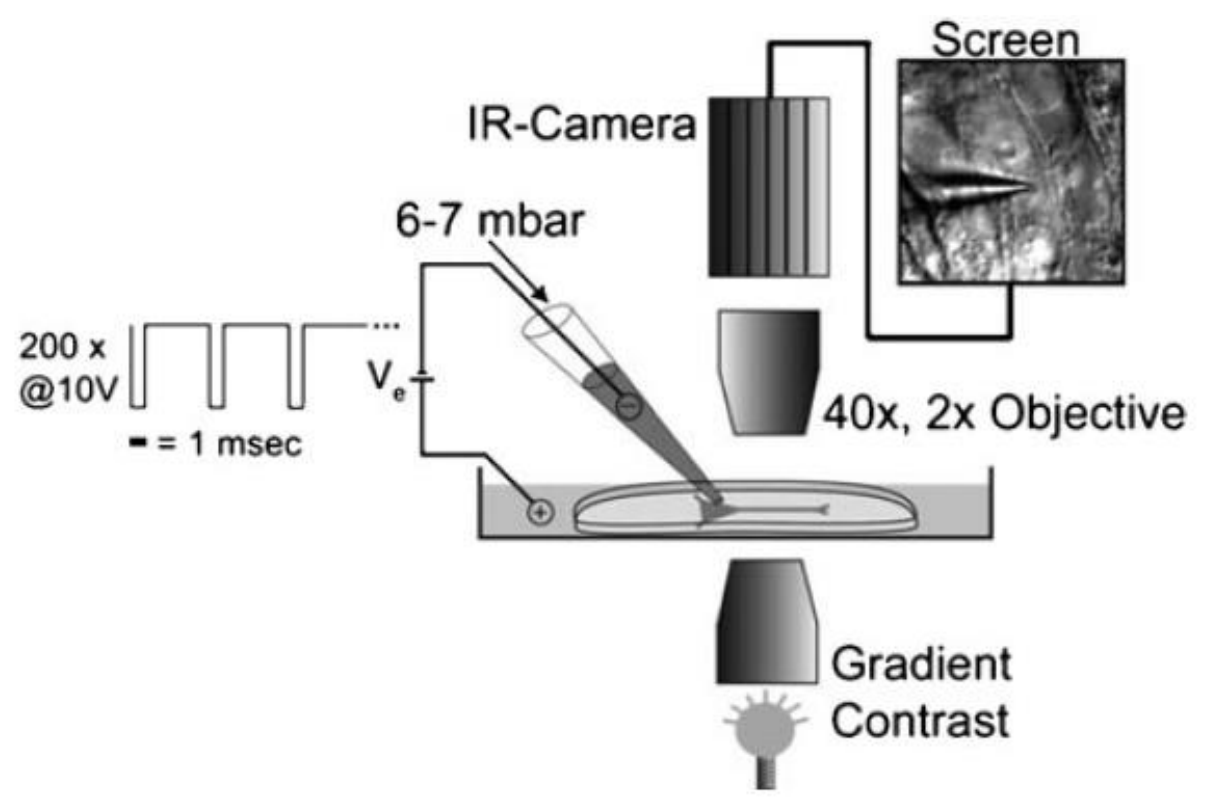

Figure 2. 7 Pipette electroporation platform setup [37]

\subsubsection{Micro-fabricated Electroporation Platform}

For bulk electroporation devices, some drawbacks, such as low cell viability rate, high stress on the cell, low transfection efficiency, and high sample contamination, have always hindered them to further development or practical application in the medical field. For micro/nano-pipette electroporation platforms, even a $50 \sim 80 \%$ transfection efficiency is a big progress compared to bulk electroporation devices. They still cannot meet the gradually growing and demanding requirement (such as high transfection efficiency, high cell viability rate, low contamination, precise dose of transferring biomolecules into cells) in electroporation application including medicine development, disease curing, and organ regrowth. In the following, several representative micro-fabricated electroporation platforms will be briefly introduced with a schematic design of their main function. In these devices, a single cell is isolated from other huge amounts of cells to obtain an inhomogeneous electric field, which was applied on a specific area of the single cell. 
Figure 2. $8 \boldsymbol{a}$ is an electroporation device made of PMMA including a channel 200 $\mu m$ high and $5 \mathrm{~mm}$ wide. This channel was coated with gold on the top and bottom. Due to the short distance between two electrodes, a relatively small voltage was applied for electroporation. A multiple patch clamp array platform was developed by Khine [38] (Figure 2. 8b), in which the constriction was utilized to block the cell moving. By applying an under pressure, the cell was trapped at the entrance of constriction due to smaller width than the diameter of the cells. In this example, less than $1 V$ was applied to create enough electroporation threshold potential. Figure $\mathbf{2 . 8 c}$ is an electroporation analysis device with a micro-hole in the silicon nitride designed and fabricated by Huang and Rubinsky [39]. The cell was captured in the micro-hole, located in the center of the middle insulator plate (silicon nitride) by pressure difference between the upper and lower fluid chamber. Two electrodes were placed $100 \mu \mathrm{m}$ apart from the middle plate for applying a small electrical field pulse to puncture a nanopore on the cell membrane. They hypothesized that a microsized pore would reduce stress on the cell and improve cell viability [23], while another group designed a different functional device for a single cell electroporation in Figure 2. $\mathbf{8 d}$. This device consists of a glass wafer coated with an electrode on its surface. It then was sealed with PDMS in order to form a cavity for loading cells. Other than the cathode electrode on the glass wafer, an extra anode electrode was added above the glass wafer to induce DNA moving towards cells once opening a pore on the cell membrane. 

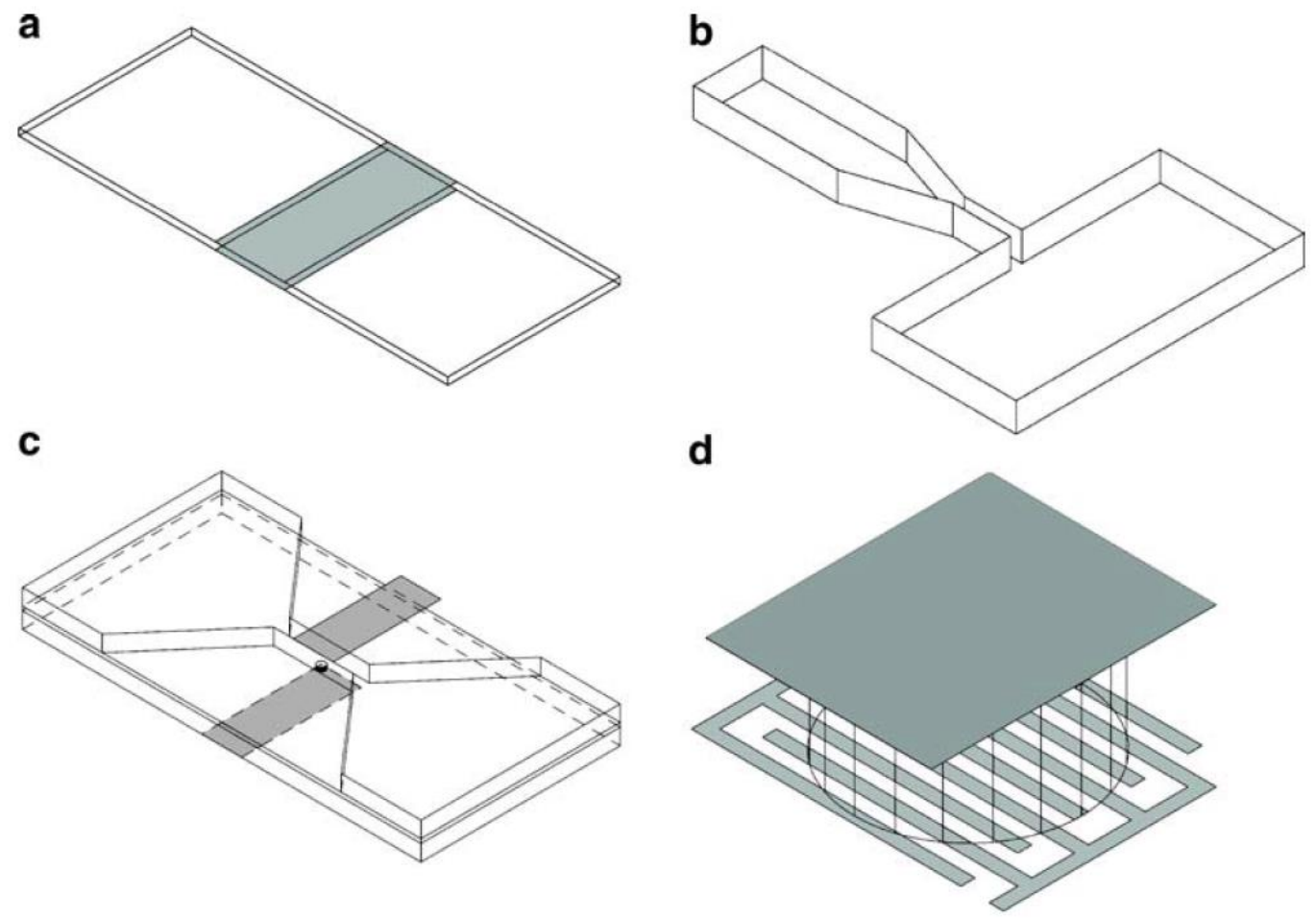

Figure 2. 8 Schematic design of different microfluidic electroporation platforms [38-43]

One representative example of cell trapping-based electroporation platforms was suggested by Khine [44]. This simple PDMS microchip used multiple narrow lateral channels to trap the cell between the $\mathrm{Ag} / \mathrm{AgCl}$ electrodes. From Figure 2. 9, the cell was trapped by negative pressure applied by an external syringe. The center circular structure was radially connected to outer surrounding micro-channels utilized as trapping sections. The width of these trapping channels is much smaller than the diameter of the cells in order to trap them. Due to this dimension limitation, the cells deform, which directly affected the efficiency of electroporation. 


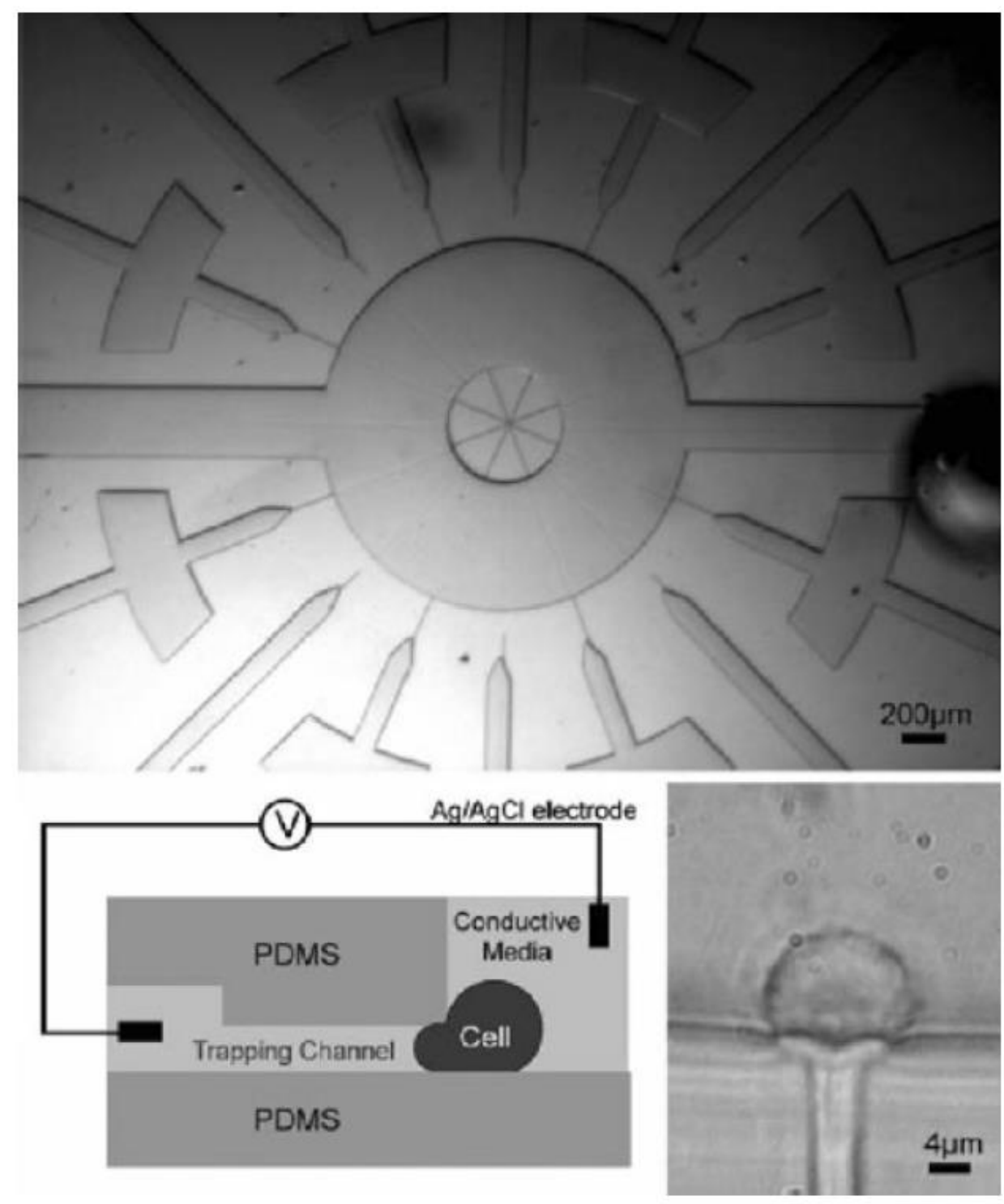

Figure 2. 9 Images and schematics of an array of narrow lateral channels for cell trapping and localized single-cell electroporation [44]

An effective design of membrane sandwich-based electroporation platform was introduced by Fei [45]. In this platform, cells were trapped on a poly (ethylene terephthalate) (PTE) membrane. The advantage of this membrane is that it has a porous surface, leading to immobilize an individual cell and apply low electrical field pulse for electroporation. 
From Figure 2. 10, we can see that it consists of two membranes: one is on the top, the other one is on the bottom for supporting an individual cell. The distance between two membranes is $10 \mu \mathrm{m}$. First, a low pulse was applied to transfer DNA moving towards the center of the system. Then, a high pulse was applied to generate enough electrical field for final electroporation.
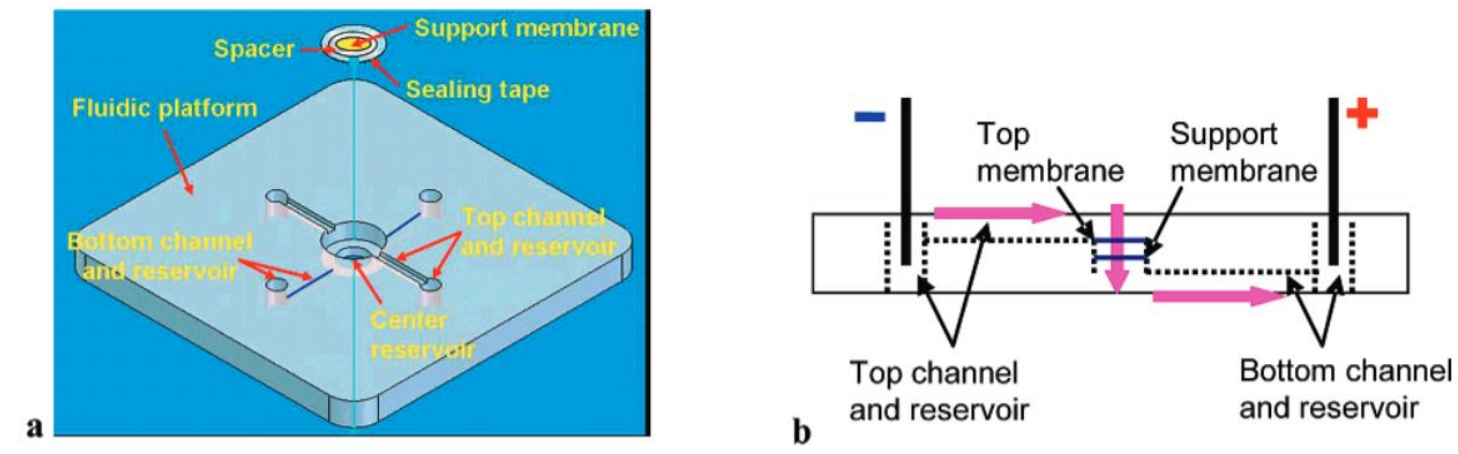

Figure 2. 10 Schematic diagram of the membrane sandwich electroporation technique (a) platform (b) schematic of DNA migration path [45]

To better understand single cell electroporation analysis, researchers had designed and developed localized single cell membrane electroporation platforms (Figure 2. 11) [16]. In this localized SCEP platform, single cells were placed on the top of two close electrodes with few micrometers distance, which can intensify the electric filed applied on the cells. During electroporation, pores were created between two electrodes to transfer biomolecules from the lower chamber into cells. Afterwards, those pores on the cell membrane resealed again once some biomolecules entered the cells through the pores. 
(a)

(b)

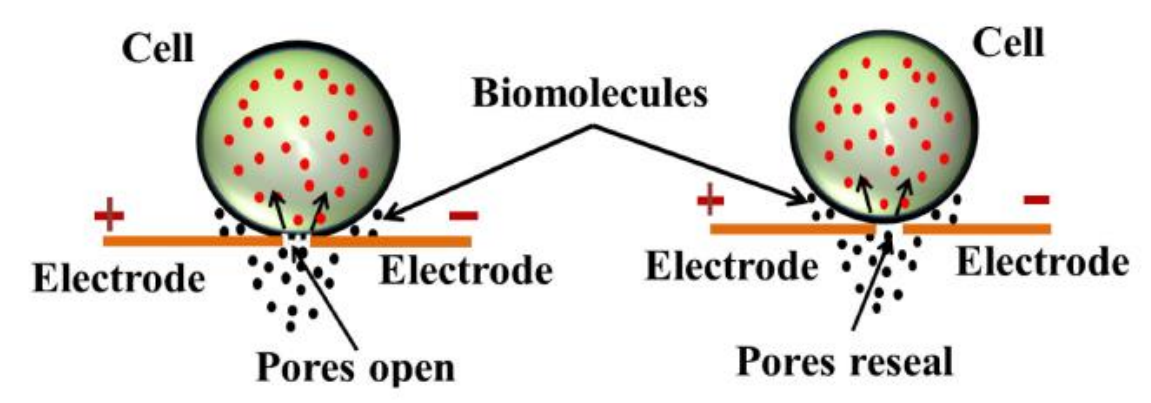

Figure 2. 11 Schematic diagram of localized SCEP platform [46]

In their study, genes/biomolecules/DNA/drugs were successfully transfected into the cells with relatively high efficiency increasing cell viability rate, reducing cellular stress, and lowering sample contamination when compared to conventional bulk or micro-pipette electroporation devices [23]. Nonetheless, these micro-fabricated electroporation platforms were plagued with Joule heating effects, an inability to precisely control the dose of transfected biomolecules and the overall cell viability was still significantly lower than normal cultured cells, which was the desired goal of these electroporation platforms [23].

\subsubsection{Nano-fabricated Electroporation Platform}

To avoid some shortcomings, such as larger Joule heating effect and lack of precisely controlling the dose of the transfecting biomolecules caused by micro-fabricated electroporation platforms, Boukany et al. $[23,36]$ fabricated electroporation devices with nanoscale channels using a DNA combing and imprinting method to investigate whether a nano-electroporation platform would enable achievement of the desired cell viability and precise control of the transfected biomolecules. These nano-electroporation platforms successfully transfected biomolecules into the cell in a controllable fashion and 
significantly improved cell viability to levels comparable to normal cell culturing techniques. In Figure 2. 12, this nanochannel electroporation device was fabricated by DNA combing and imprinting method. First, DNA was combed onto an array of microridges in order to fabricate a mold. Then gold was sputter-coated onto this mold. Then the mold was imprinted into pre-polymer resin. After curing it, the mold was peeled off, and DNA with coated gold was etched out by gold etchant. Eventually, the nanochannel as small as around $90 \mathrm{~nm}$ in diameter buried inside the bulk device was created. A theoretical electric circuit model shown in Figure 2. 13 was given by James Lee [36] from his nanofluidic electroporation system. Based on finite element simulation results in the nanofluidic electroporation system, the buffer solution in the nanochannel is equivalent as a resister. The microchannel adjacent to the nanochannel is modeled as a conductive wire with relatively low resistance. The cell membrane is separated into two parts: one close to the anode is modeled as a parallel circuit with a resistor and a capacitor, the other side membrane close to the cathode is modeled as a different value of resistor and capacitor. The cell interior is regarded as an equipotential surface. The TMP of the membrane 1 can be calculated via this equivalent electric circuit when applied voltage is given. Although their fabrication technique successfully created the desired nanochannels, several issues occurred with repeatability in fabricating the nanoscale channels, precision in controlling the nanochannel location within the device, device yield and inability to automate the process for large-scale or mass production [23]. Thus, the purpose of this study is to explore the development of a method that can repeatedly and precisely produce sub-micron scale to nanoscale channels within a PDMS-based fluidic platform, which can be utilized to perform electroporation in cells. 


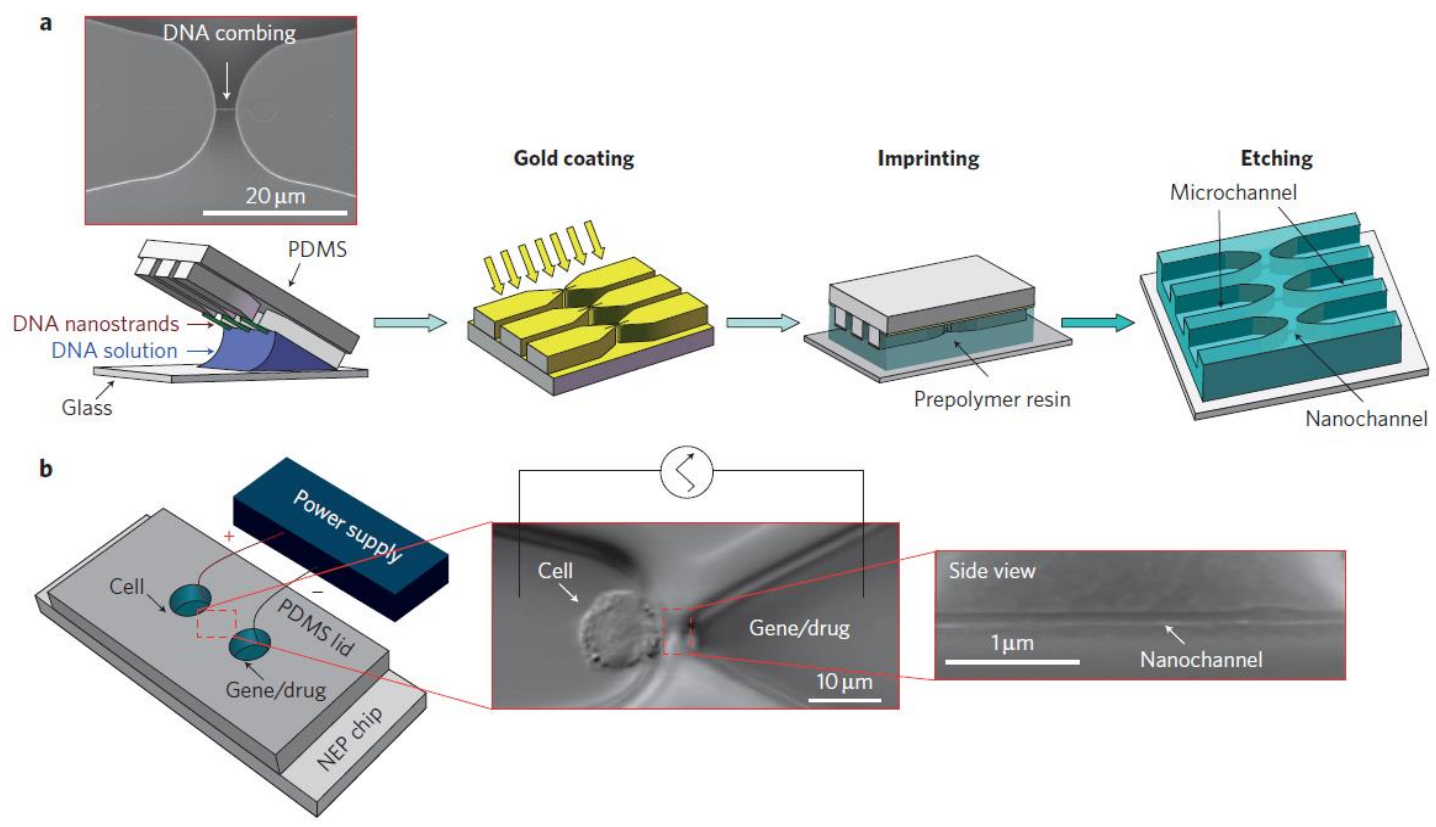

Figure 2. 12 Apparatus and operation of the NEP device.

a, Top: SEM image of a DNA nanostrand (arrow) 'combed' across two PDMS 'microridges'. Bottom: schematic showing the fabrication of the NEP chip by DCI. b, Left: schematic of an NEP chip covered by a

PDMS lid with electrodes placed in reservoirs. Middle: optical micrograph of a Jurkat cell in the left microchannel and positioned at the tip of the nanochannel using optical tweezers. The right microchannel contains gene or drugs. Right: SEM image of side view cut of a nanochannel. The nanochannel is $\sim 90 \mathrm{~nm}$ in diameter and $\sim 3 \mu \mathrm{m}$ long [36].

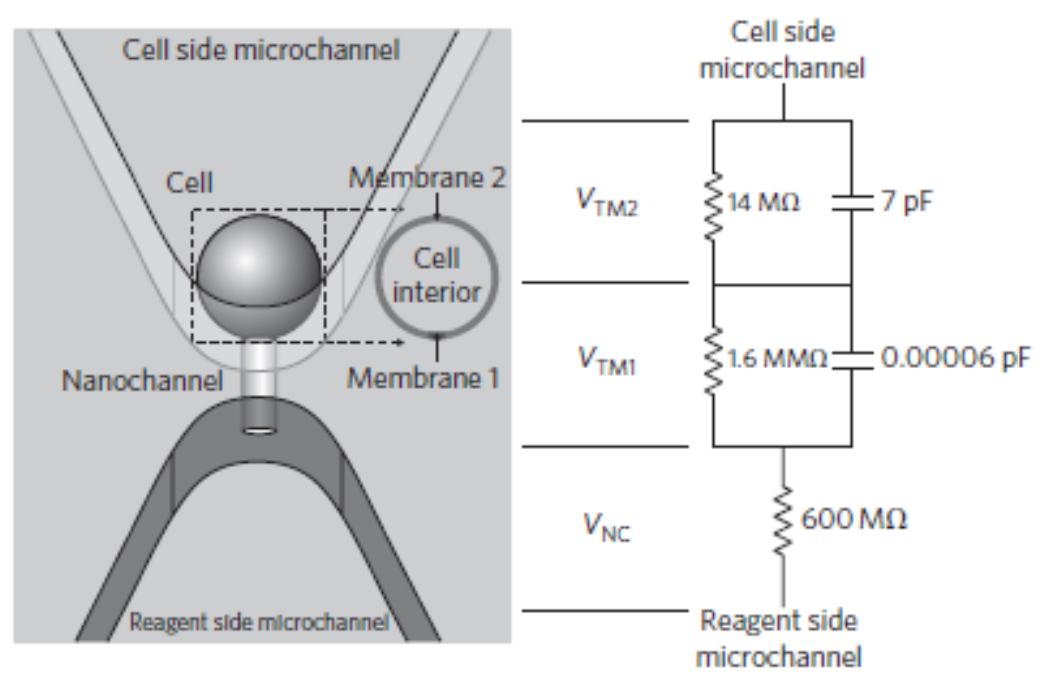

Figure 2. 13 Theoretical analysis of nanofluidic electroporation system [36] 


\subsubsection{Biomolecules Transport Mechanism in Micro/Nano-fabricated Devices}

When an electric field was applied to a fluid in the Micro- or Nano-fabricated electroporation devices, the conductivity of the fluid will increase due to ohmic heating caused by elevated temperature of the fluid with electric field [47]. Biomolecules needed to be transfected into cells in the channels of microfluidic devices will be charged by the applied electric field in the conductive fluid. Unlike conventional electroporation devices, the driven force needed to move cells was from electrokinetic transportation rather than external applied pressure, which was the motion of a charged cell in an electric field since the cells themselves were charged due to their composition of the cell membrane. This motion was composed of two phenomena: electroosmotic flow and electrophoretic transport [48].

Electroosmotic flow is defined by a bulk liquid motion induced by an externally applied electric field across the fluid conduit, which takes place inside the fluidic channels. For a conventional capillary, the tube was filled with electrolyte fluid with $\mathrm{pH}$ value greater than 2. The capillary with negative charges ionized from conventional tube walls was distributed around the surface of walls. Due to attraction, the ions with positive charges from the fluid are attached to the wall surface, which is composed of a rigid layer. Because the chemical equilibrium between a solid surface and an electrolyte solution typically leads to the interface acquiring a net fixed electrical charge, a layer of mobile ions, which is an electrical double layer or Debye layer, forms in the region near the interface [49]. When an electric field is applied at the two ends of the tube, the net charge in the Debye layer is induced to migrate towards the cathode electrode by Coulomb force, which is the 
phenomenon of electroosmotic flow. The velocity of electroosmotic flow is defined by the Smoluchowski equation:

$$
V_{E O}=\frac{\varepsilon \zeta E}{4 \pi \eta}
$$

where $V_{E O}$ is electroosmotic flow velocity, $\varepsilon$ is the permittivity of fluid, $\zeta$ is the zeta potential, $E$ is the electric field strength, and $\eta$ is the viscosity of fluid [50]. The zeta potential is the potential difference across the diffusion layer, which is defined as a layer starting from just past the rigid layer until the middle of capillary where positive and negative ions are nearly equal in concentration. The zeta potential varied with location, which means that the location near the rigid layer has greater zeta potential than that of other places. According to Equation (2-5), the ions close to the wall have the greatest velocity. Its value relates to what tube wall materials are, the fluid's $\mathrm{pH}$ value, the fluid's temperature, and the ionic concentration of fluids.

Dispersed particles or biomolecules within surrounding fluids with charges move towards their counter-electrode under the electric field force. This motion is called electrophoresis, which is relative to fluid electroosmotic flow. The electric field force applying on the particle is expressed as:

$$
F=q E
$$

where $q$ is the particle's charge [51]. By Stokes' law, this force is equal to frictional force, which is defined as [52]:

$$
q E=6 \pi \eta r V_{E P}
$$


where $r$ is the radius of particle, $V_{E P}$ is the particle's relative velocity to the flowing fluid. Solved above equation, we get:

$$
V_{E P}=\frac{q E}{6 \pi \eta r}
$$

where $V_{E P}=\mu E, \mu$ is the electrophoretic mobility [53].

The absolute velocity of a charged biomolecule in the bulk fluid is the superposition of electroosmotic and electrophoretic velocities if we assume that fluid is neutrally charged and a small Debye layer relative to channel size [48]. This absolute velocity of biomolecules becomes very significant to the study of the duration time of cell transfection in the case that the length of channel, electric field strength, fluid properties, and physical properties of the channel are known since we can determine the final travel time of charged biomolecules through the channel with above equations and physical parameters.

\subsection{Electroporation Devices Fabrication Techniques}

\subsubsection{BioMEMS}

Due to Micro-Electro-Mechanical Systems (MEMS) technology providing possibility of creating two dimensional or three dimensional complicated structures with high precision, MEMS technology and its incorporation into different materials such as polymers, dielectric materials, and metals for bioengineering applications have developed a new promising field, "BioMEMS" [54-57]. By combining many characteristics, including being capable of controlling their chemical, electrical, mechanical, and physical characteristics on a nanoscale level, there is a growing interest in integrating living cells or biomolecules with micro-fabricated devices and using microfabrication technology for micro total analysis systems ( $\mu \mathrm{TAS}$ ) [58], biosensors [59], stents [60], drug delivery [61], 
microneedles [62], and injectable biomolecules [63]. In a word, MEMS creates a miniaturized and specific scaffold on which researchers could utilize this unique microenvironment to do biological and biomedical analysis. In addition, BioMEMS have the advantages of micro/nanoscale size, being capable of operating on micro/nanoscale time, and comprehensive characteristics over other types of biological devices for some certain application, such as electroporation platforms.

\subsubsection{Micro/Nanofabricated Electroporation Platform Fabrication}

A number of different methods for creating microfluidic devices have been previously developed including direct fabrication techniques (photolithography (including $\mathrm{X}$-ray), laser photoablation, laser micromachining), and prototyping techniques such as injection molding, hot embossing, and soft lithography [64, 65]. Among these techniques, silicon, polymer, and glass were utilized to meet different specific requirements for certain types of biological and biomedical applications. In the following, some representative microfluidic device fabrication techniques will be introduced.

Due to high accuracy, high resolution, ultrashort timescale and ultrahigh laser intensity coupled to the electronic system, the laser micromachining technique has been widely incorporated into the fabrication of microfluidic devices [66]. According to [67], micromachining was using a micro-scale size drilling and tuning tool and miniature milling to fabricate microfluidic devices (Figure 2. 14). A stereo microscope was used to monitor milling process. Even though the advantages introduced above for micromachining are over many other traditional methods, it is limited in high cost, centralized labor, optical opacity, and difficulty in component incorporation into silicon or glass, and requiring highly specialized expertise and equipment [68]. 


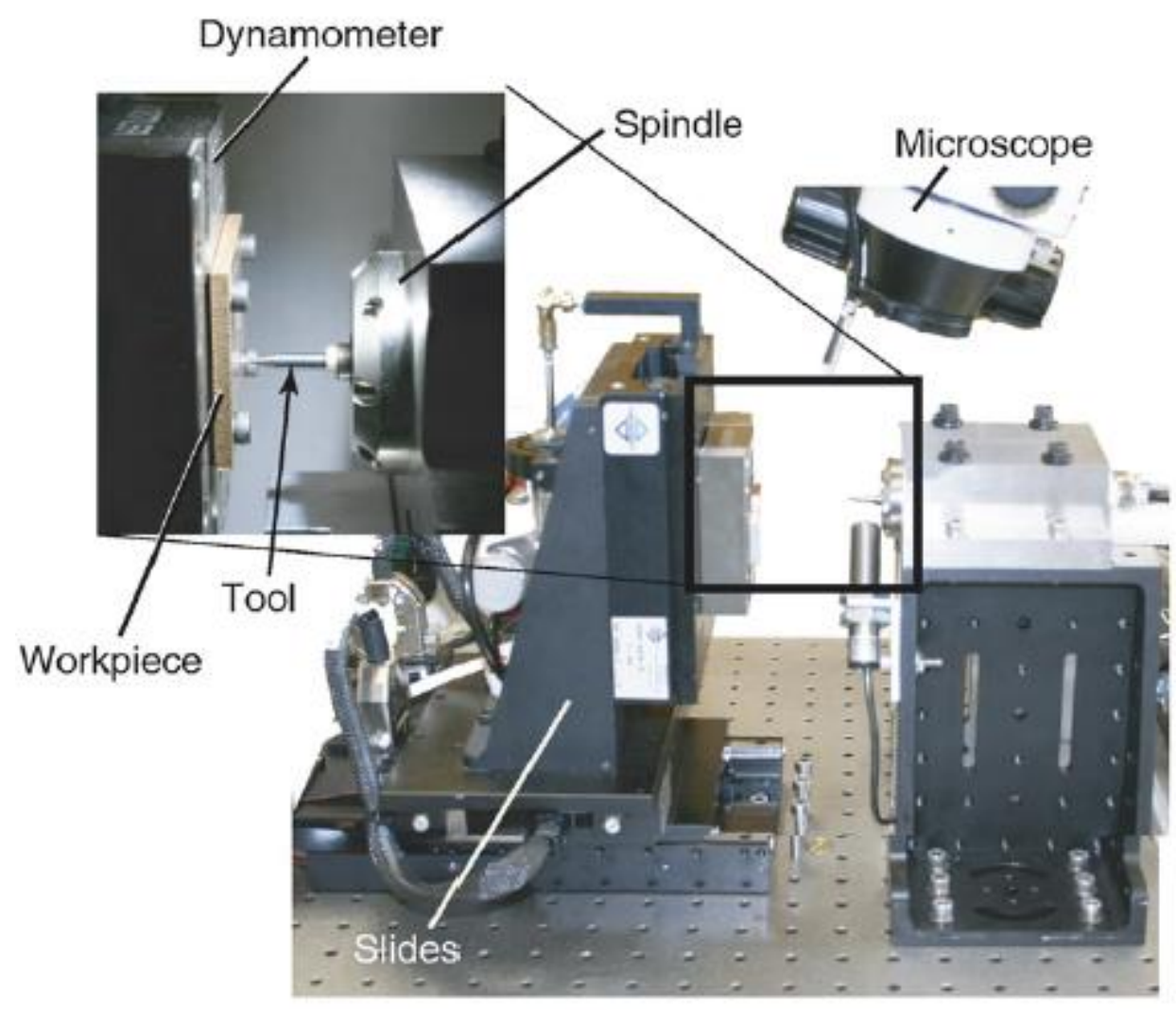

Figure 2. 14 Micromilling machine setup [69]

Compared to the micromachining method of fabricating microfluidic devices, injection molding based on laboratory methods had the advantage of low cost, ease of operation, and flexibility (Figure 2. 15). First, the mold was fabricated by common methods, such as hot embossing and photolithography [70]. Then liquid polymer or molten plastic was injected into the cavity with mold inside. After that, a vacuum pump was utilized to eliminate the air bubbles of liquid polymer or molten plastic. Last, after baking and cool down, the specified microfluidic devices were fabricated, followed by peeling off polymer from the mold. The disadvantages of this method lie in low resolution and poor choices of materials for injection when considering compatibility with future application, 
while for the industry method of mold injection, it has the advantages of high throughput, fast fabrication, and large-volume production.

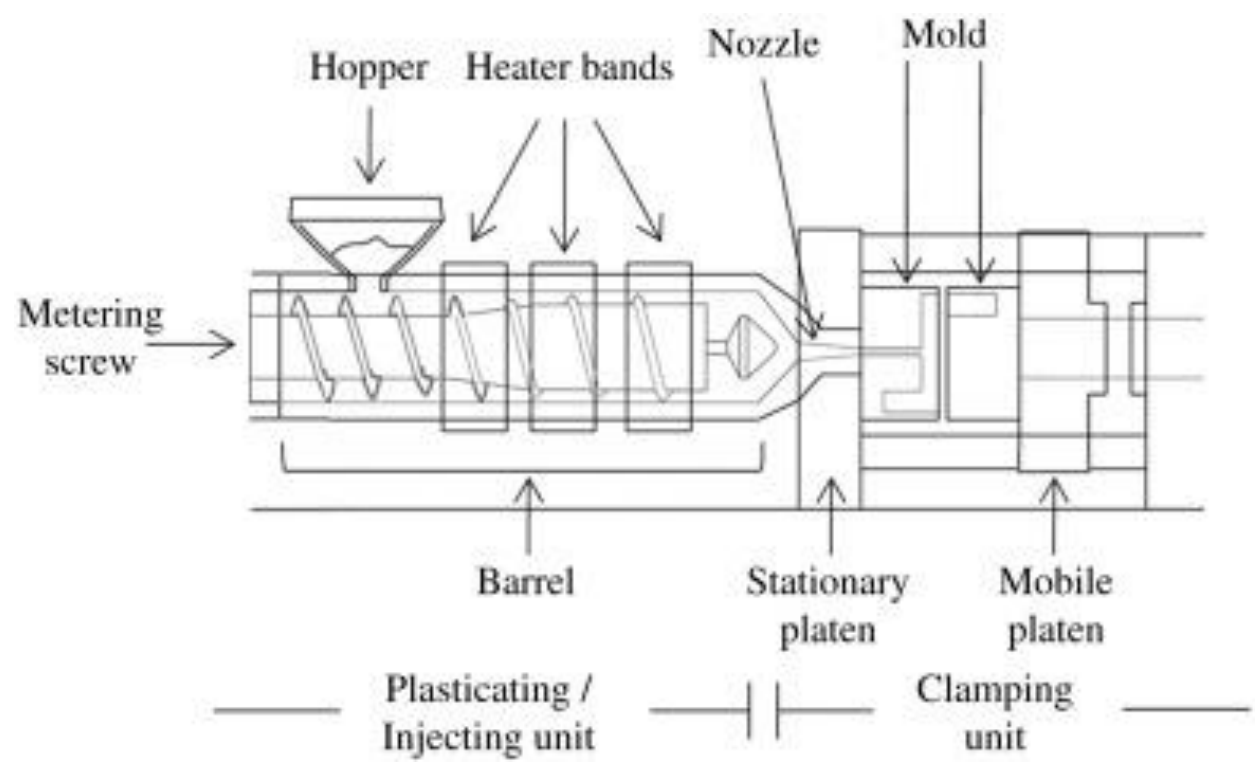

Figure 2. 15 Injection molding schematic design [71]

Using soft photolithography, simple micro-channels can be generated on a substrate layer via micro-patterns produced on an elastomeric surface [72]. The advantages of soft lithography include easy to learn, straightforward to apply, and low-cost [73, 74]. Like micromachining, the mold masters of this technique were fabricated by traditional photolithography. Typically, polydimethylsiloxane (PDMS) is an appropriate original master. Usually, contact printing and capillary molding are the two most common soft photolithography methods (Figure 2. 16) [75-78]. Contact printing is a direct patterning method involving applying an elastomeric stamp and is able to generate a non-structured, chemically modified surface $[75,76]$, while capillary molding uses an elastomeric mold which is placed on a substrate covering a layer of polymer, which fabricates topographically modified physical microfluidic devices [77, 78]. Actually, capillary molding is an advanced soft lithography method since higher resolution and much more 
complicated microstructures have been created. But the disadvantages of soft photolithography are complexity in fabricating multilayer structures due to alignment, limited to amorphous materials, and lack of temperature range control [73, 74].

In sum, although the techniques introduced above for fabricating different types of electroporation platforms are viable, there are issues with repeatability and larger scale production. Thus, a new technique such as nanofiber drawing by the direct-write method combined with thin dry film photolithography, film-to-film bonding, and replica molding needs to be explored to fabricate an ideal 3-D nanofluidic platform for nanoscale cellular electroporation study.
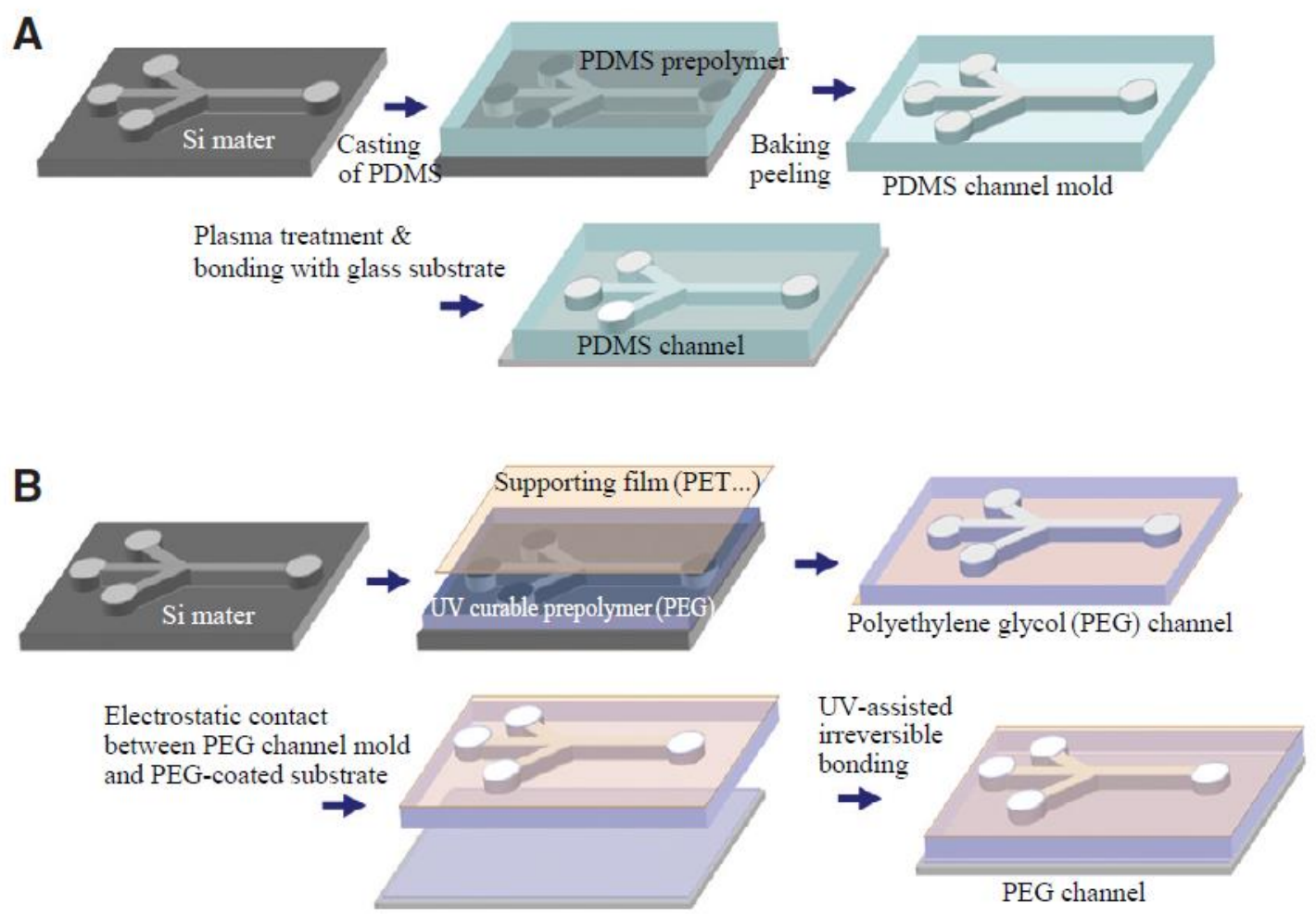

Figure 2. 16 Schematic representation of microfluidic channel fabrication using soft lithography [72] 


\subsection{Polymer Fibers Fabrication Techniques}

\subsubsection{Wet Spinning, Dry Spinning and Electrospinning}

Over the past several decades, a variety of polymer fiber fabrication techniques, such as wet spinning, dry spinning, melt spinning, and electrospinning, have been employed to create novel polymer fiber structures with diverse and robust biological, chemical, electrical, and mechanical properties [79-90]. Wet spinning involves using polymer, which needs to be dissolved in a solvent to make the polymer solution first. Then, the spinneret was submerged in a tank loading with a specific chemical bath that makes the polymer solution to precipitate, then solidify. A different drying method was utilized in the dry spinning technique to solidify the polymer solution. Usually, a stream of air or inert gas acted as a drying agent to evaporate the solvent in the polymer solution.

For melt spinning, different types of polymers or polymer components were utilized, which can be melt to create polymer fibers after cooling down and being extruded from an orifice plate $[48,91-95]$. And very fine polymer fibers can be drawn using electrospinning (Figure 2. 17), which used an electrical charge to propel polymer solution from a syringe needle to a copper plate, which is a polymer fiber collector. Eventually, fibers ranging from tens of nanometers to several microns randomly distributed onto the collector substrate via characterizing the experimental parameters including amplitude of electric filed applied, polymer solution viscosity, surface tension, electrical conductivity and so on. Although some strides had been taken to align these micro/nanoscale fibers by using patterned electrodes, conductive substrates, and disc collectors [96-100], this technique still lacks the ability to generate individual and suspended micro/nanoscale fibers. 


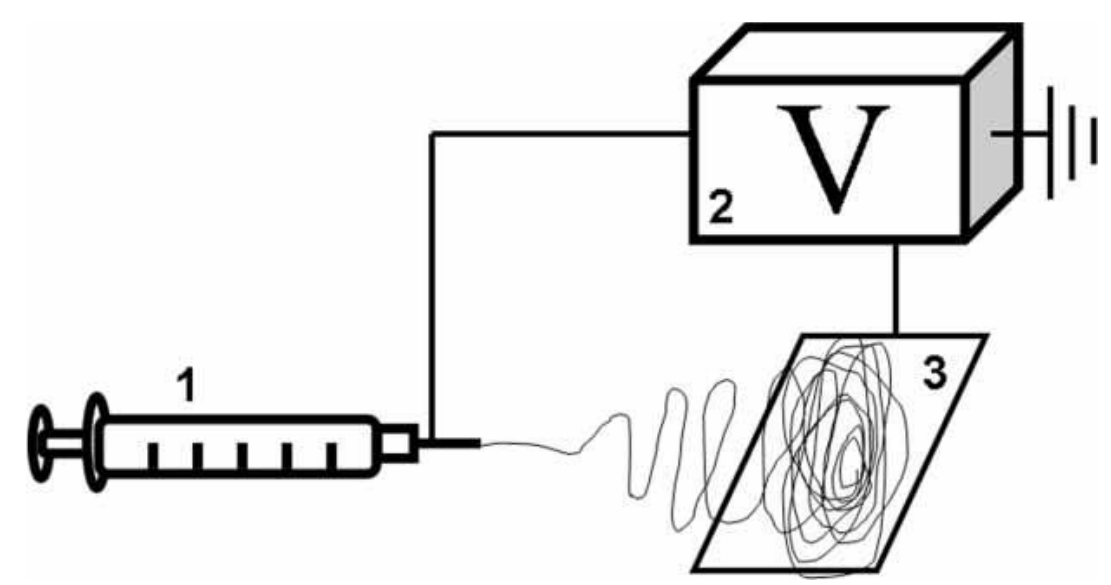

Figure 2.17 Schematic of a typical electrospinning apparatus [96]

Although these spinning techniques were capable of generating micro/nanoscale fibers, they were limited in their ability to precisely create individual, suspended, and oriented fibers in three dimensions (Figure 2. 18). In addition, these techniques were restricted in their dimensional range for fiber fabrication; specifically, fibers produced via wet and dry spinning varied in diameter from tens to hundreds of microns, while electrospinning yielded fibers with diameter ranging from tens of nanometers to a single micron depending on the presetting parameters [101]. 

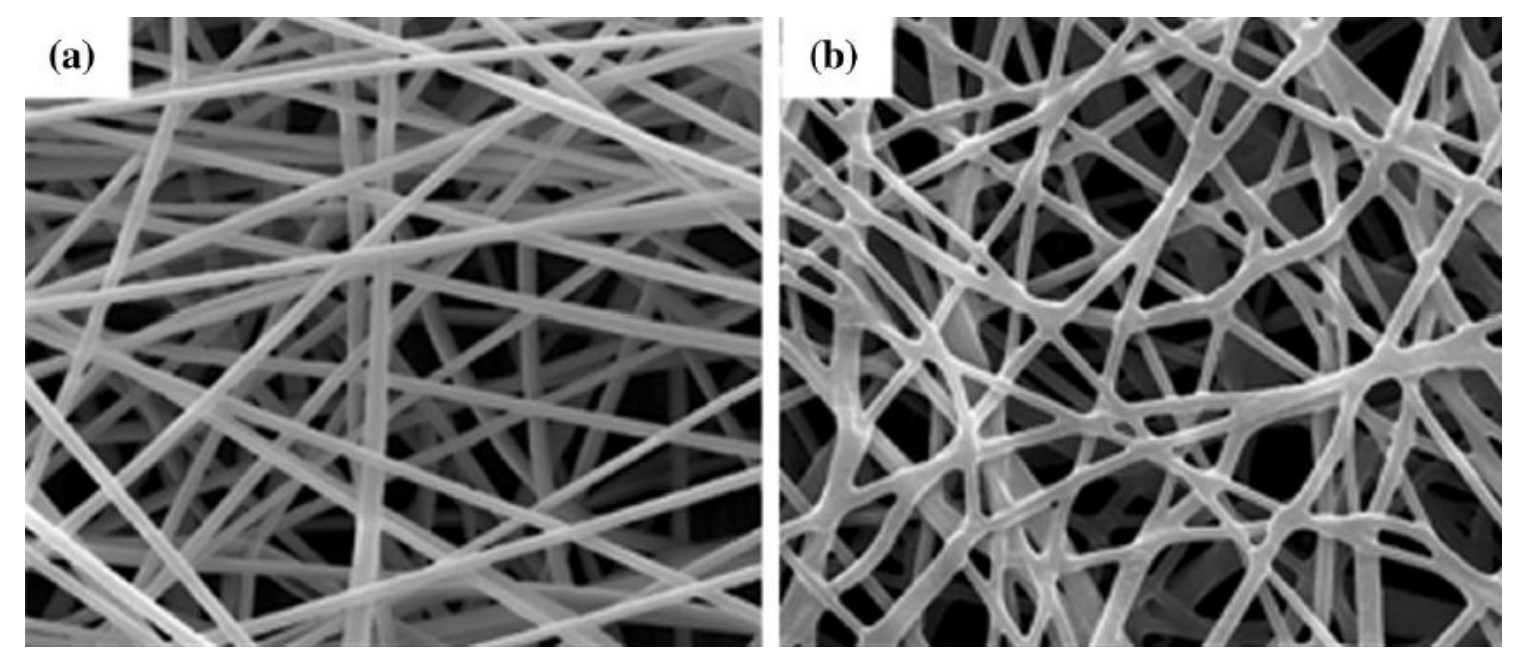

Figure 2. 18 Drawn fibers resting on the collector [102]

\subsubsection{AFM Tip and Hand-brush Based Fiber Fabrication Methods}

To overcome the above drawbacks, a new category of fiber fabrication techniques was developed by drawing or manually brushing liquid fibers onto a predetermined position of the substrate. Harfenist et al. used nano-manipulating instruments like AFM tip to create suspended fiber bridges with diameters ranging from $50 \mathrm{~nm}$ to $20 \mu \mathrm{m}$ onto the micro-fabricated array of tips on a prefabricated substrate (Figure 2. 19) [90]. The novelty of this new direct fiber drawing method is to form and pattern fibers into three dimensional suspended geometries compared to planar lithography, and dry, wet, and electrospinning techniques. In addition, this method is fast with only one step and is easy to operate without using spinneret, chemical bath, or electrical charge. 

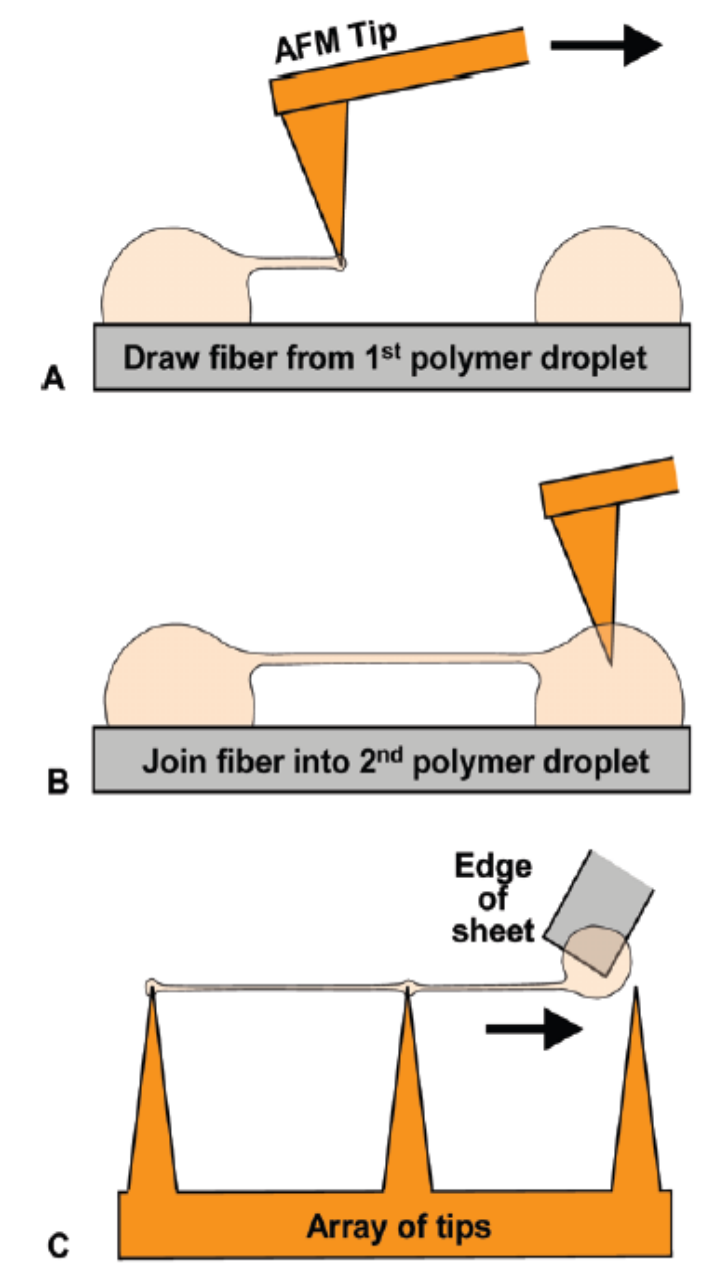

Figure 2. 19 AFM tip drawing suspended fiber structures [90]

Pabba manually hand-brushed the polymer solutions across the micro-structured surfaces using a simple edge faced applicator that had been coated with polymer solutions to fabricate parallel and suspended arrays of micro/nanoscale fibers (Figure 2. 20) [103]. This manually hand-brushed method extends above direct drawing by utilizing different materials, such as biocompatible, biodegradable, nanocomposite, and amphiphilic copolymers [103]. In addition, low concentrations polymer solution was applied in drawing fiber structures on prefabricated micro-pillars using this manually hand-brushed method. 


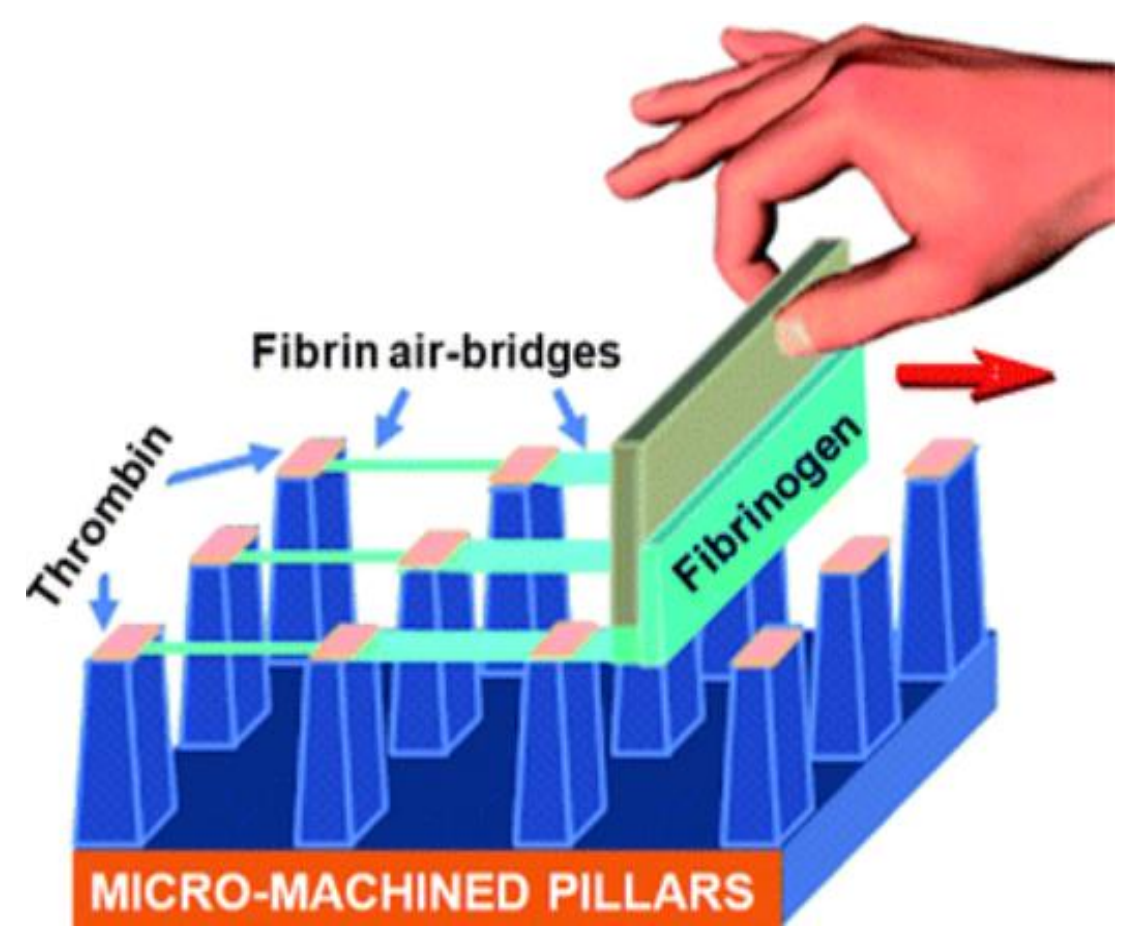

Figure 2. 20 Direct drawing suspended fiber structures [90]

Although these two techniques precisely generate the fibers at predefined locations, they are incapable of creating fibers with repeatable and controllable dimensions with predefined combinations of controlling factors. The geometries are also limited by the prefabricated micro-pillar pattern of the substrate. Even though these two methods have the advantages of ease to operate and fast to fabricate fibers, the cost of creating oriented and suspended fiber structures is relatively high due to using AFM tips or an array of prefabricated tips because of the written substrate to control the fiber orientation in three 


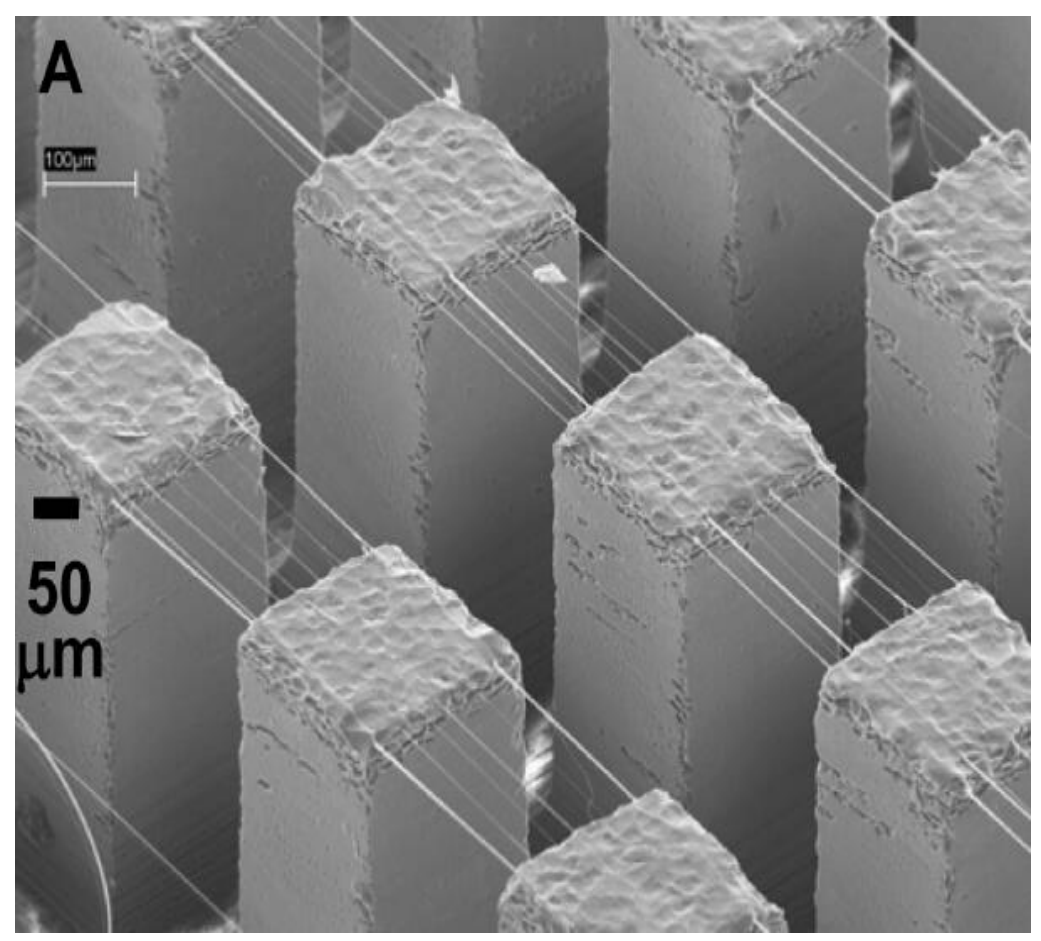

Figure 2. 21 SEM image of suspended and oriented fiber structures [90]

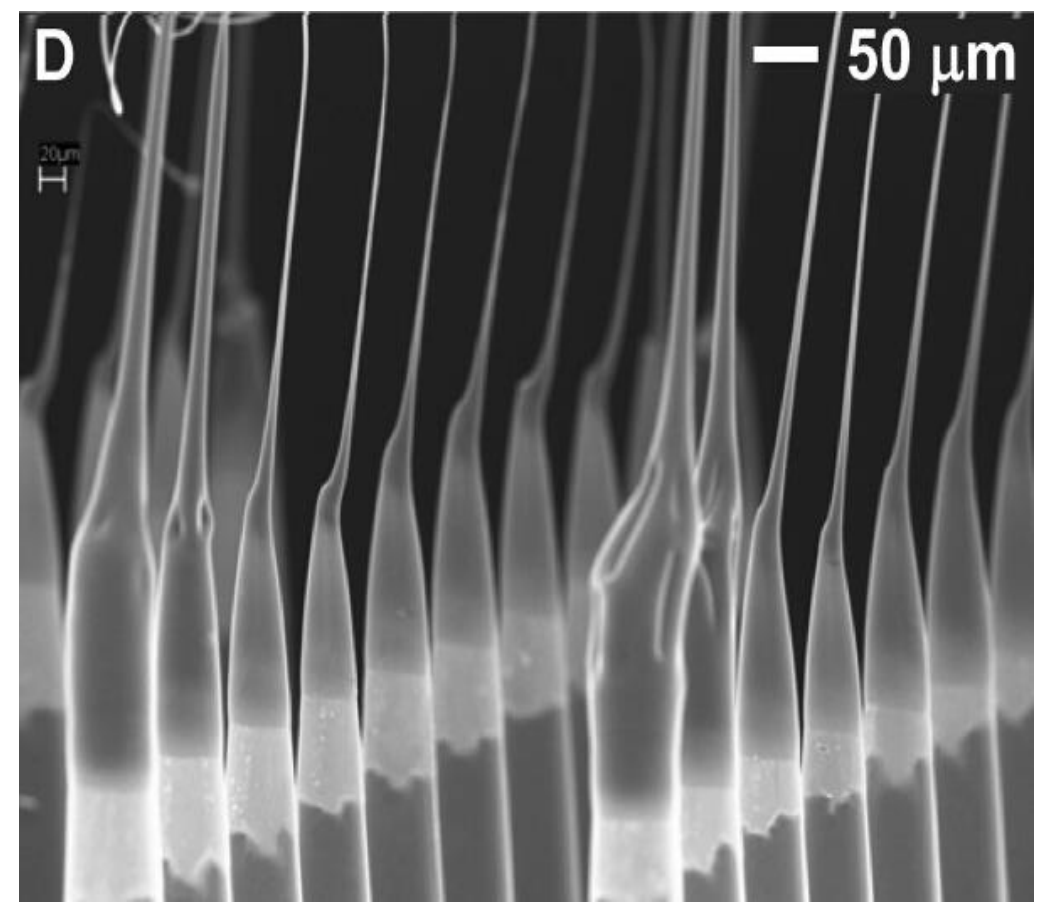

Figure 2. 22 Fibers drawn between a sharp tip Array and a layers of polymer [90] 
dimensions. Individual fibers' diameter is different (Figure 2. 21, Figure 2. 22), which are coming from the same experimental parameters. It is difficult to repeatedly draw the same or close dimension fibers with the same experimental parameters using above fiber creation methods.

\subsubsection{Principle of Polymer Solution Capillary Thinning and Breakup}

Elasticity, viscosity and inertial stress are significant in resisting the tendency of polymer solution capillarity and controlling its 'necking' or thinning process when an initially stable polymer solution column or thread broke-up into a smaller droplet [104]. The inertial stress, inertia, and viscosity are mainly coming from the extensional deformation of the polymer solution's internal microstructure within the column or thread. Experimental and theoretical analysis has been studied in the field of capillary-driven thinning and break-up in order to quantitatively and quantificationally measure the thinning and break-up process [105-107]. Capillary pressures can be explained in thinning of liquid filament and break-up processes which arise from surface tension and the curvature of the interface [108]. The viscosity of polymer solutions would increase during the thinning or break-up process. The key point is to maintain the polymer solutions' viscosity as low as

possible to slow down and even stop capillary thinning from proceeding to break up into two individual filaments from a single column or thread [103]. Physical and mechanical properties of polymer solutions can be depicted by surface tension, viscosity, and evaporation rate which play an important role in thinning polymer filaments.

Surface tension is an important factor in describing liquid capillarity. Surface tension is the elastic tendency of liquids which makes them acquire the least surface area possible [109]. For example, at liquid-air interfaces, water molecules have greater 
attraction to each other due to surface tension than the air molecules have. For polymer liquids, surface tension is responsible for the shape of droplets because the droplets of liquid tend to be pulled into a spherical shape by the imbalance in cohesive forces of the surface layer. Each molecule in the bulk of polymer liquid is pulled equally in each direction by neighboring liquid molecules which results in a total net force of zero, while the molecules of polymer liquid at the surface do not have the same molecules like the bulk at each direction of them and therefore are pulled inwards to form spherical shape droplet. Wilhelmy technique was exploited to measure the surface tensions of polymer solutions by dipping a glass rod into test polymer solutions [110]. The micromilling machine was mounted to the glass rod and it precisely controlled the XYZ coordinates to touch the surface of the test solutions in order to make the contact angle of fluid on the rod approaching zero. A high resolution balance was used to measure the force change due to surface tension of test fluid on the tip of the rod.

For polymer solution liquid, viscosity is a measure of its resistance to gradual deformation, which corresponds to "thickness," by shear stress or tensile stress [111]. Viscosity arises from collisions between neighboring particles in the polymer solutions that are moving at different velocities. Viscosity of a polymer solution is dependent on molecular weight of the dissolved polymer [112]. Usually, a polymer solution with high viscosity has large molecular weight when dissolved [113, 114]. As a result, polymerization of mono-polymer or lower molecular weight polymer can be utilized to increase viscosity during the process of capillarity and break-up of polymer column and thread [103]. Polymer solution viscosities were measured by LVDV-II+ and RVDV-II+ viscometer (Brookfield, Middleboro, MA) interfaced with a cone-and-plate (CP-52). Low 
viscosity fluids were measured by the LVDV-II+ viscometer since it has limited measurement range, while high viscosity fluids were measured by the RVDV-II+ viscometer. The principle of the viscometer lies in measuring the resistance exerted by the test fluid in rotation in a cone-and-plate in contact with fluid. With the predefined parameters, the viscometer would directly show current fluid viscosity by converting the resistance torque into current viscosity.

The evaporation rate of the polymer solution's solvent plays a significant role in polymer capillary thinning or break-up [115], because over time, it can affect the viscosity of the polymer solution. Low volatility solvent would be a good choice to dissolve polymer in order to decrease the evaporation rate and maintain the viscosity as much as possible. Mass transfer coefficients of the polymer solutions were measured by the thermogravimetric analysis (TGA, TA Instruments TGA 2950, New Castle, DE), which could be utilized to record high-precision weight measurements at different temperatures [110]. Compressed air and cooling gas were vented through the chamber in case of accumulating solvent vapors and acquiring high temperature after shutting down the device.

\subsubsection{Dimensionless Parameters of Polymer Solution Filament Formation}

In order to create polymer filaments with repeatable and controllable dimensions with our robotic dispensing system, it is important to understand the physics of polymer solution during the capillary process. As discussed above, elasticity, viscosity, and inertial pressure from the polymer solution's internal microstructure will stop or retard its capillarity. Kolte and Szabo [116] and McKinley and Tripathi [117] summarized the early models explaining that the surface tension of the new fibers will thin until capillary break- 
up when drawing filaments from the polymer solutions [48]. The mathematical model indicating that break-up time has direct relationship with the viscosity and the surface tension of the polymer solution is given as follows:

$$
D(t)=D_{1}-\frac{(2 X-1)}{3} \frac{\sigma}{\eta} t
$$

where $D(t)$ is the diameter of polymer solution fiber as a function of time $t, D_{1}$ is the initial diameter of the fiber, $X$ is a constant equal to $0.7127, \sigma$ is the surface tension of the polymer solution, and $\eta$ is the Newtonian viscosity. Although this mathematical equation illustrated some relationship between filament break-up time and the polymer solutions' viscosity and surface tension, it is limited to just a linear relationship description among those factors, and does not provide the final filament diameter with precision, but zero, when the filament ultimately breaks up.

A more persuasive model with the evaporation rate was presented by Tripathi [118] in order to overcome above shortcomings. In this model, the evaporation rate of the polymer solvent was introduced to illustrate the complicated relationship among viscosity, surface tension, and volatility of solvent affecting the final diameter of filament. An equilibrium diameter $D_{\infty}$ after solidification and thinning without any breaking is shown as follows:

$$
D_{\infty}=D_{1} e^{-0.035 / P}
$$

where $P$ is a dimensionless processability parameter and $P=\frac{\eta \chi}{\sigma}, \chi$ is the evaporation rate (solvent mass transfer coefficient). This equation suggests that filament diameter will minimize to a steady-state, non-zero value for a constant-length filament of the Newtonian polymer solution [119]. 
When the filament drawing rate is substantially higher than the capillary thinning rate and the solidification rate, the above processability parameter model is not applicable [48]. In order to solve this problem, some additional dimensionless parameters were added to account for the thinning of the fibers and the solidification mechanics. In sum, the function of polymer droplet thinning is expressed by five variables: surface tension, viscosity, fiber length, drawing rate, and mass transfer coefficients of polymer solvent. In addition to the processability parameter, two additional dimensionless parameters were regarded as important factors affecting the filament thinning process and the final equilibrium diameter, which are capillary number $\left(C_{a}\right)$ and aspect ratio $(\Lambda)$ [48]. Ca explains the non-instantaneous nature of the direct-write method via the robotic dispensing system, in which creation of a single fiber can only take from less than one second to several seconds [119]. Ca is expressed as:

$$
C_{a}=\frac{\eta U}{\sigma}
$$

where $U$ is the drawing rate. $\mathrm{Ca}$ indicates the ratio of the drawing rate to the surface tensiondriven thinning rate. With a high capillary number, the majority of the surface tensiondriven thinning occurs after extension is complete due to the fast drawing speed, while some fiber thinning occurs during extension which probably results in break-up for low capillary number. Aspect ratio is defined as follows:

$$
\Lambda=\frac{L}{D_{0}}
$$

where $L$ is the fiber length after drawing, $D_{0}$ is the initial diameter of the polymer solution before beginning to draw. For our case, $D_{0}$ is equal to the inner diameter of the dispensing needle tip. 


\subsubsection{Direct Write Fibers via Micromilling Machine}

To provide control of fiber orientation and dimensionality more precisely in the 3D space, our group had developed a self-assembled or "direct-write" fiber fabrication process that directly ejects a polymeric material out of a hollow capillary and then draws the individual filaments which thin and solidify into predictable fiber diameters by exploiting surface tension-driven fluid mechanics (Figure 2. 23) [119]. Our initial directwrite system for increasing the level of control of fiber position and diameter consisted of a custom fabricated spring loaded syringe dispensing system attached to the head of a custom made Ultra-High Precision Micromilling Machine (Dover Instruments, Boxborough, MA) (Figure 2. 24) [110]. The UHPMM had a stage positional resolution of $1.25 \mathrm{~nm}$ in the $\mathrm{X}$ and $\mathrm{Y}$ directions and $20 \mathrm{~nm}$ in the $\mathrm{Z}$ direction that was programmatically controlled to create micron and sub-micron scale wires and structures. First, polymer solution was expelled from syringe needle by gravity of the wrench weight applied on the top of the syringe, then the syringe was lowered to just the initiating point

of contact with the substrate. Subsequently, syringe was translated to the terminating point to draw polymer filament. During this process, surface tension of the polymer solution drives the polymer filament to neck down. In the meantime, solvent will evaporate until the fiber solidifies during the fiber elongation process. Eventually, expelled polymer solution was contacting the terminating point to form a suspended and individual fiber. 

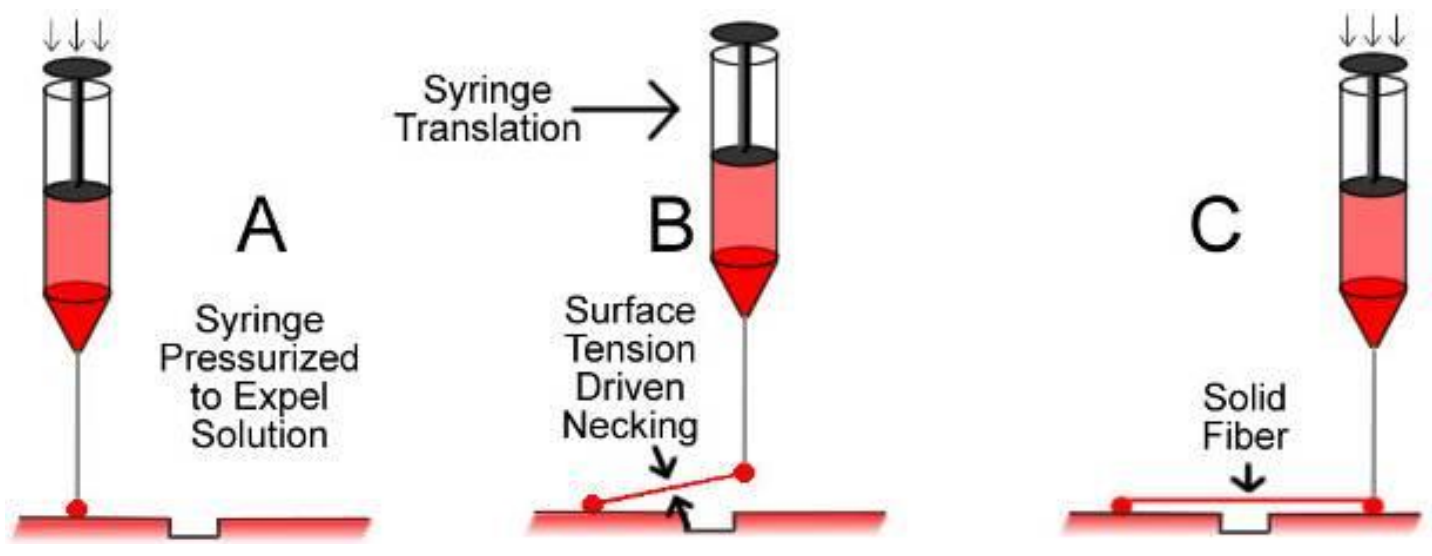

Figure 2. 23 Schematic illustrating the direct-write drawing of fibers [119]

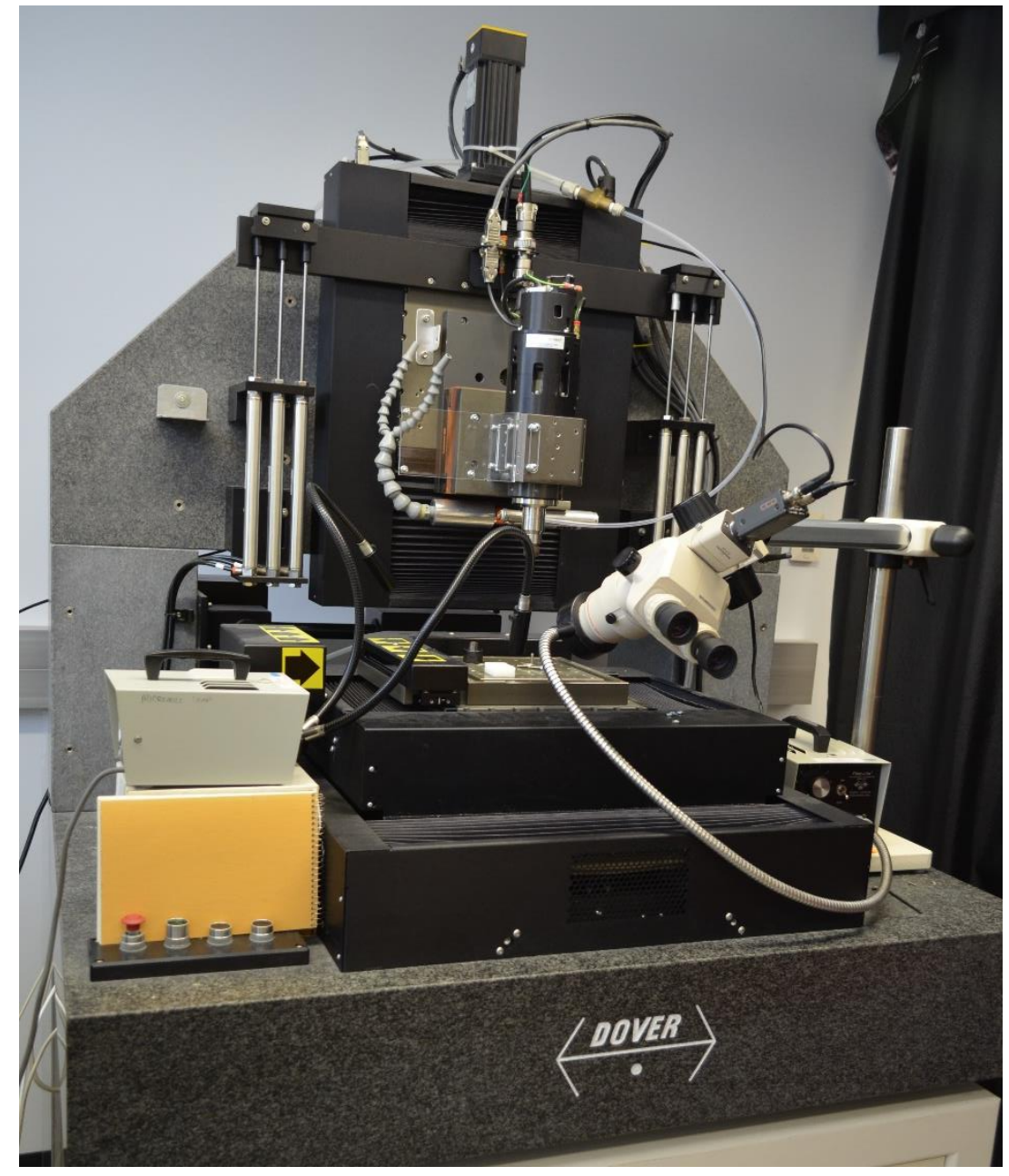

Figure 2. 24 Micro-Milling Machine and its constituent parts [110] 
One limitation of this particular direct-write system was that it lacks flow control of the polymer solution through the needle tip. Although the spring-loaded dispensing system successfully generated constant flow through the tip, a continuously expanding spherical bead of polymer solution was created at the outlet of the syringe tip, which varied in size and volume depending on environmental conditions. Furthermore, fibers' diameter was not small enough to meet the requirement of fabricating micro/nanofluidic electroporation platform.

\subsubsection{Direct Write Fibers via Robotic Dispensing System}

The inconsistency of this source bead impacted the ability of the micromilling machine system to repeatedly fabricate fibers of a prescribed diameter. Albeit, structures were successfully generated using this direct-write process, enhancement of the process by increasing control of polymer solution flow would allow for more precise, prescribed fiber diameters through regulation of the bead size at the syringe tip. Characterization and optimization of this direct-writing fiber process, using a new three-axis robotic dispensing system, would allow us to write much smaller, more repeatable and controllable micro/submicron/nanoscale complicated fiber structures in three dimensions. Compared to micromilling machine, programming the trajectory of complicated fiber structures is much easier to achieve in the three-axis robotic system. Thus, this dissertation will focus on implementation of a 3-axis automated dispensing system with pneumatically actuated dispenser valve to precisely control the polymer solution flow rate and tip bead size to create prescribed, complex three-dimensional structures in order to fabricate an ideal nanofluidic electroporation platform. 


\section{CHAPTER 3 METHODS AND MATERIALS}

In this project, a new micro/nanofluidic platform was designed, fabricated and evaluated to demonstrate proof-of-concept for performing electroporation in cells. The fabrication procedure consisted of implementing new methodologies and a 3-axis robotic dispensing system for creating micro/nanochannels within a micro/nanofluidic platform. The 3-axis robotic dispensing system was characterized and optimized to repeatedly generate prescribed micro/nano fibers. Upon completion of the fabrication process, the micro/nanofluidic platform was evaluated to demonstrate feasibility in electroporating cells via the transport of a fluorescent dye into the cell. A detailed description of the methods, processes and techniques utilized in this study have been provided below.

\subsection{Micro/Nanofluidic Platform Design}

A design similar to Boukany's nano-electroporation device [36] was chosen to take advantage of the superior attributes of their device over bulk or micro-electroporation platforms. The design for this cell electroporation device consisted of two, tapered microchambers in juxtaposition to one another with a gap (or "dam") having a separation distance of 10 microns (Figure 3. 1). Micro/nanofluidic channels were created within the dam of the PDMS devices with either a microchannel $($ channel design diameter $=1 \mu \mathrm{m})$, sub-microchannel $($ channel design diameter $=500 \mathrm{~nm})$, or nanochannel $($ channel design diameter $=300 \mathrm{~nm}$ ) separating the two micro-chambers. Each micro-chamber was designed with a taper that reduced the width of the chamber from $10 \mathrm{~mm}$ down to a width of $240 \mu \mathrm{m}$ 
to direct the movement of the cell to the micro/nanochannel. Moreover, the microchambers had a depth of $30 \mu \mathrm{m}$ and a length of $17 \mathrm{~mm}$ to maintain a large enough media volume during the cell electroporation procedure. In addition, an inlet port $(\mathrm{D}=1 \mathrm{~mm})$ was included to enable the transfer of cell solutions or solutions of biomolecules into the two micro-chambers, respectively, and to provide electrode access to the micro-chambers during the electroporation procedure. The distance between the two inlet ports was set to 5 $m m$ to allow sufficient working space while also minimizing the distance, thereby reducing the resistance, between the two electrodes when the high electric field gradient was applied during electroporation. The overall outer dimensions of the PDMS substrate was designed to be $40 \mathrm{~mm}$ long, $20 \mathrm{~mm}$ wide, and $3 \mathrm{~mm}$ high.

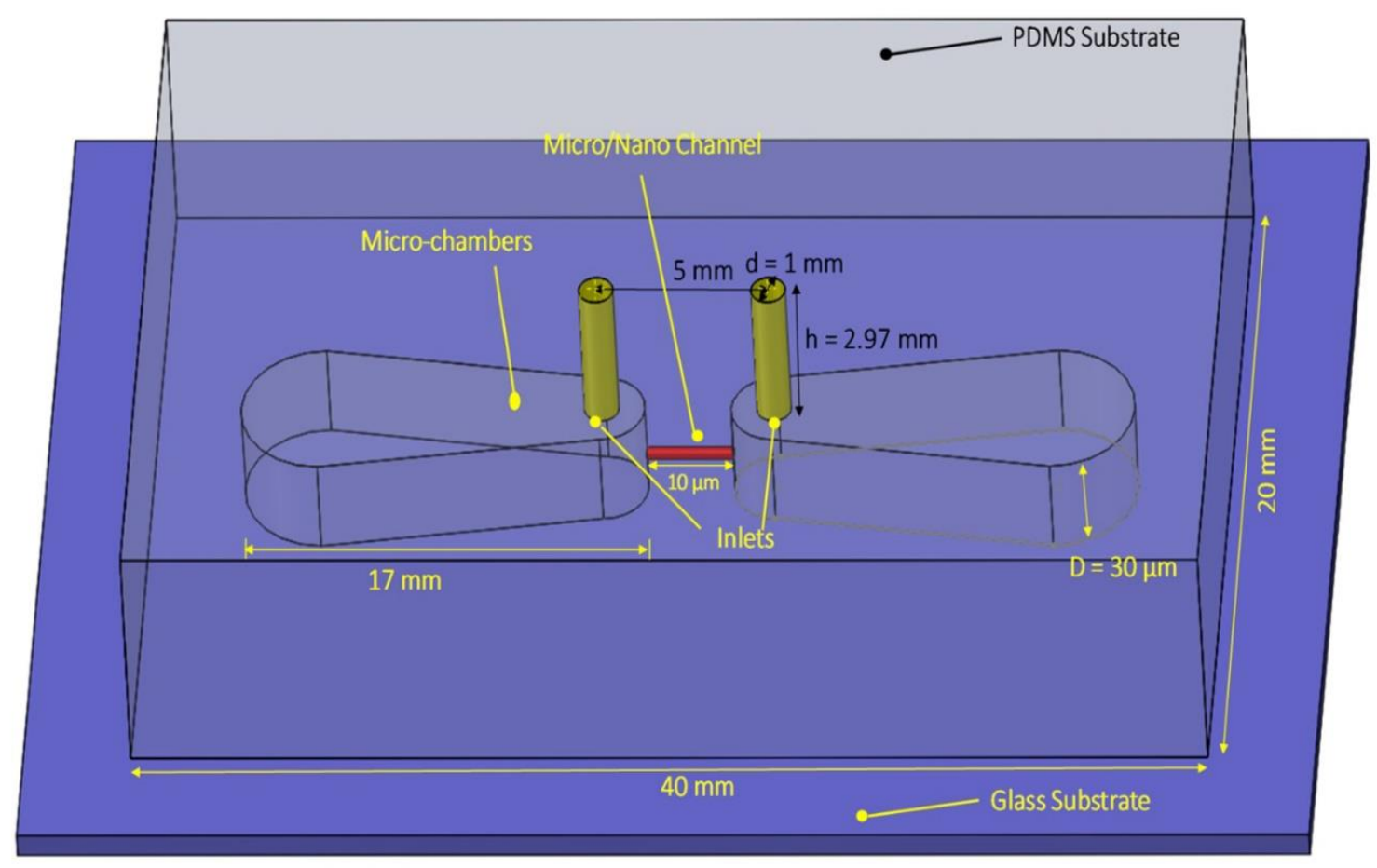

Figure 3. 1 Schematic diagram of the design of the Micro/Nanofluidic electroporation device 


\subsection{Direct-write Micro/Nano PMMA Fibers}

In order to create the micro/nanochannels within the micro/nanofluidic platform, a previously developed direct-write method [120] was implemented using a 3-axis robotic dispensing system. The 3-axis robotic dispensing system was characterized and optimized to enable the fabrication of prescribed Polymethylmethacrylate (PMMA) micro/nanofibers. The robotic dispensing system, together with the characterization process, have been described below.

\subsubsection{3-Axis Robotic Dispensing System}

The prescribed micro/nanofibers were drawn on the micro-patterned substrate using a 3-axis robotic dispensing system (model JR 2203N, Nordson, Westlake, Ohio). The robotic dispensing system (Nordson Corporation, Westlake, Ohio) included a dispensing valve, valve controller (Valvemate 7100 Dispense Valve Controller, Nordson Corporation, Westlake, Ohio), 3-axis positioning system (JR2000N Desktop Robot, Janome Sewing Machine Co., Tokyo, Japan), USB-microscope, feedback controlled heater, and a sealed enclosure (Figure 3. 2). The dispensing valve and 3-axis positioning system with spatial resolution of 5 microns in the $\mathrm{X}, \mathrm{Y}$, and $\mathrm{Z}$ directions were controlled by JR C-Points software. The JR C-Points software controlled the positioning of the fibers' initiation and termination location (Figure 3. 3), syringe needle travel velocity (referred to as feed rates) (Figure 3. 4), vertical distance of the syringe needle from the substrate, and dispense time (Figure 3. 5). The dispense time referred to the time that the PMMA solution was pressurized in the needle before fiber drawing began. A valve controller was utilized to purge the needle tip and push the polymer solutions from the barrel until the PMMA was 
discharged from syringe needle tip (Figure 3. 6). The USB- microscope enabled visualization and recording of the entire direct-write process. This helped monitor the process by identifying when droplet accumulation occurred on the needle tip during the fiber drawing process and determined when fiber break-up ensued. The direct write system operated in a polycarbonate enclosure to prevent environmental air flow/circulation over the workspace, which caused undesired fiber breakage during the writing process. A feedback-controlled heater was located in the plastic sealed enclosure to maintain a constant temperature and ensure a uniform evaporation rate of the polymer solvent.

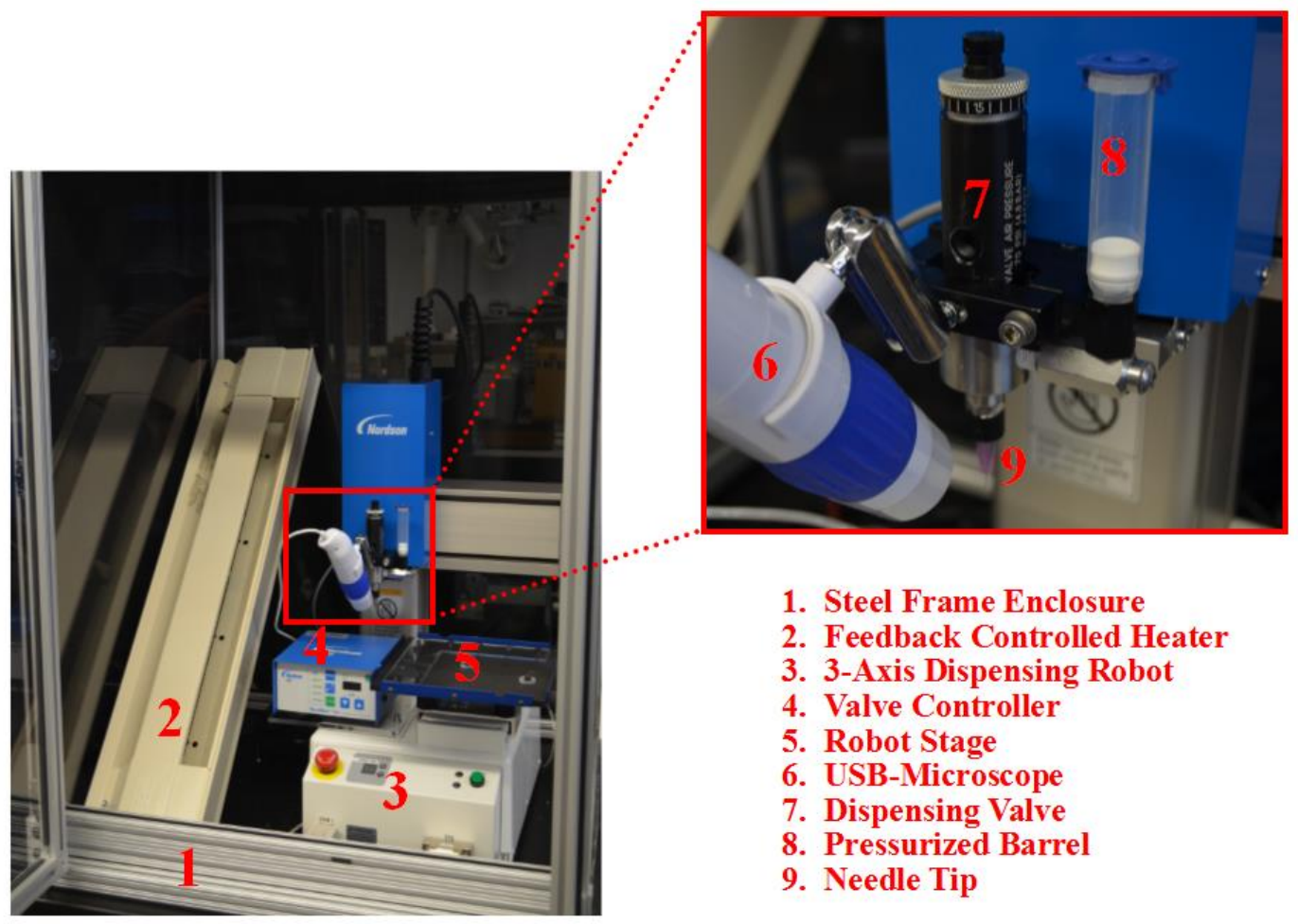

Figure 3. 2 Images of the 3-axis robotic dispensing system and its constituent parts: Left image is of the entire system housed in the plastic enclosure; and, Right image is an enlarged image of the dispensing mechanism and imaging system. 


\begin{tabular}{|c|c|}
\hline 1 & 2 \\
\hline Point Dispense & Point Dispense \\
\hline GD.DOD & 60.000 \\
\hline 105.250 & 140.250 \\
\hline 24.550 & 24.550 \\
\hline- & - \\
\hline- & - \\
\hline
\end{tabular}

Figure 3. 3 Image of the display panel in the JR-C Points software for defining the fiber length

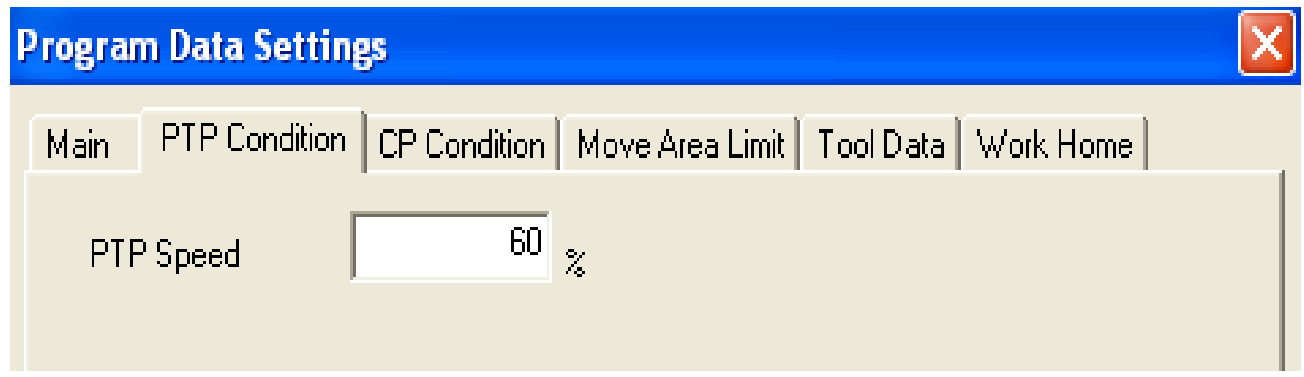

Figure 3. 4 Image of the display panel in the JR-C Points software for defining the point-to-point travel velocity, i.e. the feed rate.

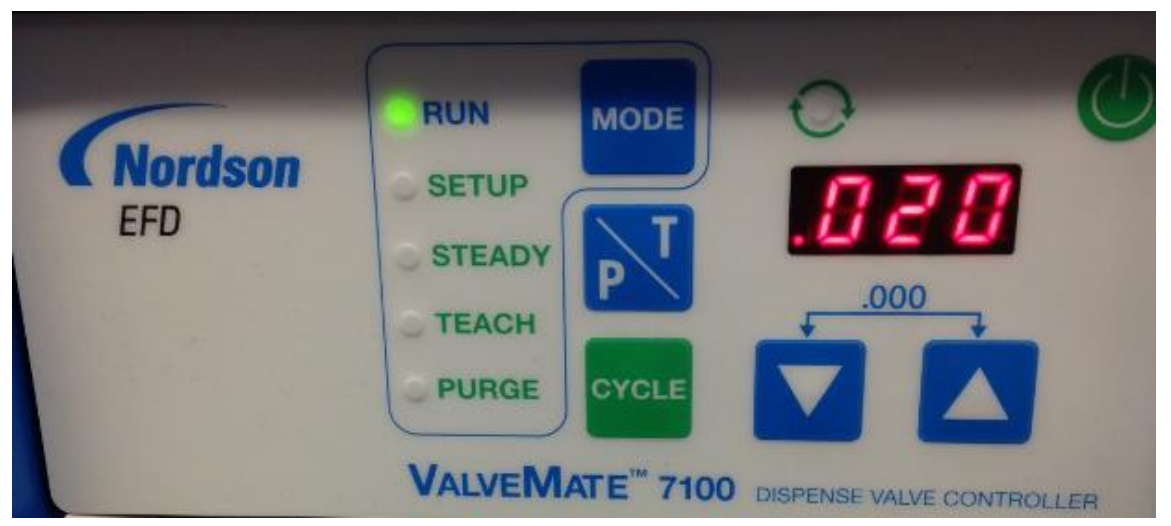

Figure 3. 5 Control panel display on the 3-axis robotic system with the dispense time for the valve controller displayed digitally in the upper right side of the image. 


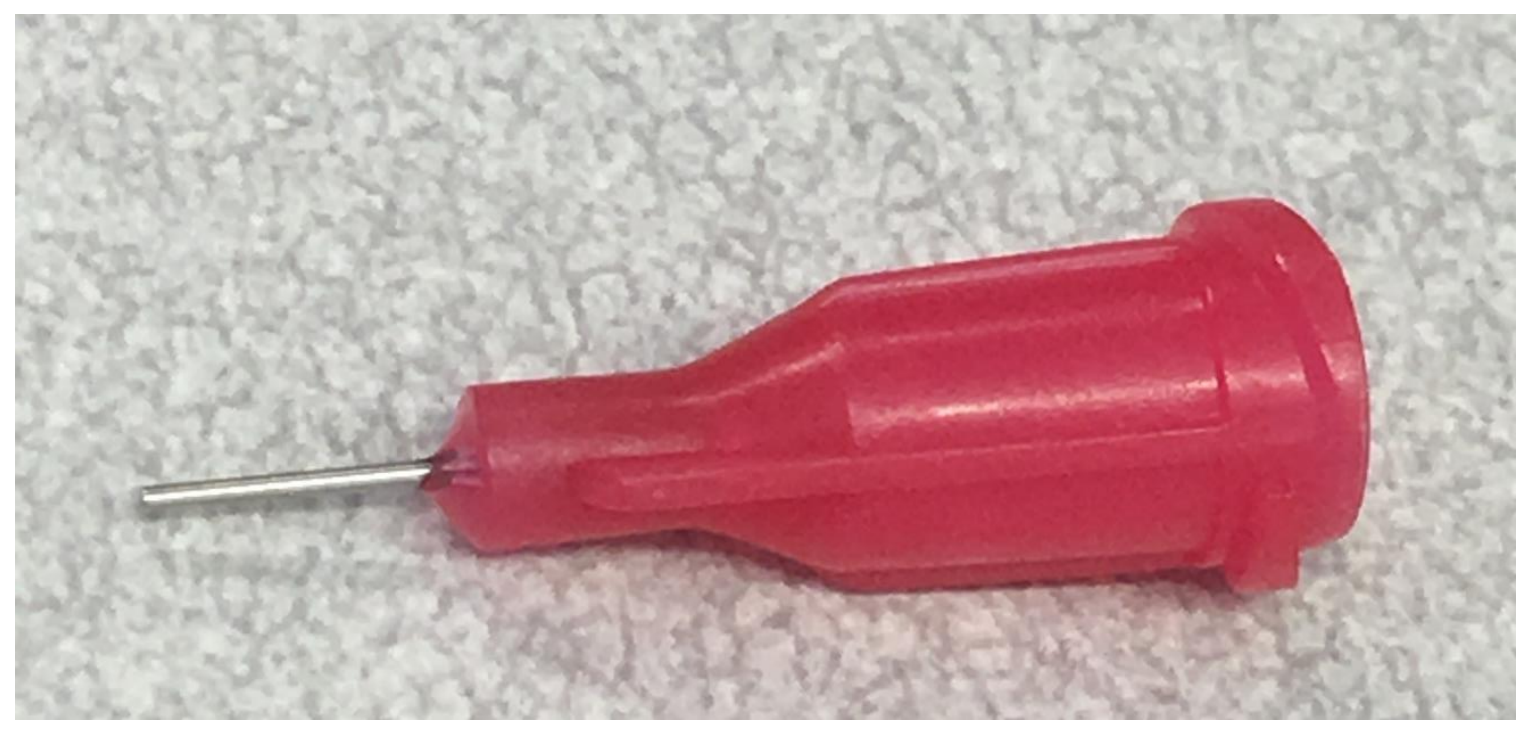

Figure 3. 6 Image of a typical syringe needle tip (25 Gauge needle shown here).

\subsubsection{Overview of the 3-axis Robotic Dispensing System Direct-write Process}

Prior to performing the direct-write process, PMMA solutions were prepared by dispersing PMMA powder (Sigma-Aldrich, MW = 996g/mol) into chlorobenzene solvent at weight concentrations varying from $19 \%$ to $27 \%$ in $1 \%$ increments. First, the weight of the solvents and PMMA powder were calculated according to the desired specific weight percentage. Then, the desired amount of polymer powder or resin was placed in a glass vial using a Pasteur pipet. Next, the chlorobenzene solvent was transferred into the glass vial using a pipette. Subsequently, the vial was mixed for 1 minute using a vortex shaker and ultrasonically processed for five hours to completely dissolve the polymer powder/resin. Two techniques were utilized to identify whether the PMMA was completely dissolved: 1) the solutions were allowed to set for several days after ultrasonication and their transparency was observed; and, 2) the loading vial was placed upside down to watch the solution flow with a constant speed until total exposure of the bottom vial was observed 
without any remnant resin present. The PMMA polymer solution was then loaded into the syringe.

PMMA sample loading into the 3-axis dispensing system consisted of five detailed steps. First, $3 \mathrm{~mL}$ of polymer solutions were loaded into a syringe barrel, and a piston was placed into that syringe barrel to eliminate inconsistent pneumatic pressure distribution. Subsequently, an inlet line adapter was twisted onto the syringe barrel in order to connect the syringe to the line source for air. Then, the desired gauge size for the precision needle tip was selected and mounted to the dispensing valve to perform the experiment. The "mode" switch on the dispense controller panel (Figure 3.5) was pressed to the purge state and "cycle" button was pushed to fill the dispense valve with the polymer solution until exposure of the PMMA solution was observed at the needle tip. Finally, the residual polymer solution was wiped from the tip prior to the programmed fiber writing procedure since the extra polymer solutions adversely affected the final fiber diameter.

The direct-write process consisted of four main steps as illustrated in Figure 3. 7. The syringe needle was first positioned to a predefined spot on the substrate and lifted up $1 \mathrm{~mm}$ from this initial touching point. The syringe needle was then laterally translated with a constant travelling velocity to the termination location on the other side of the substrate. During this step, the PMMA solution droplet thinned and elongated by surface tensiondriven necking. Finally, the single fiber direct-write process was completed by lowering the syringe needle down $1 \mathrm{~mm}$ onto the terminating position of the substrate. The rate at which the thinning filament solidified was dependent on the solvent's evaporation rate and environmental temperature. If the filament solidified too fast, it would break prior to completion of the elongation process. 


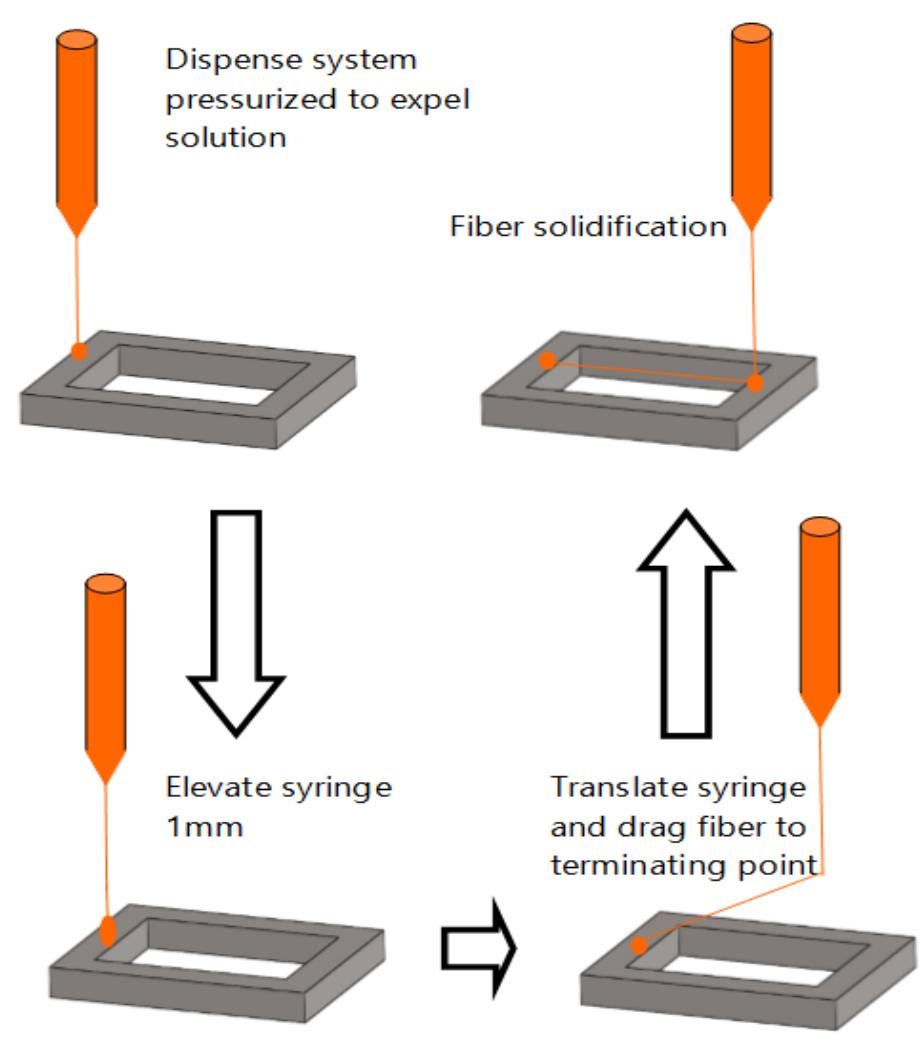

Figure 3. 7 Schematic of the direct-write process via the Robotic Dispensing System.

Once the direct-write process was completed, it was necessary to perform a cleaning process for the whole dispensing system to prepare for the next experiment with a different polymer solution or polymer concentration. To begin cleaning the system, the needle valve was disassembled according to manufacturer's protocol. Subsequently, all the metal parts were immersed in a beaker of acetone and subjected to an ultrasonic bath for 30 minutes to remove all polymer residues. Last, all parts were rinsed under flowing DI water and dried with compressed air. 


\subsubsection{Direct-write Procedure for Complex Suspended Fiber Structures}

Complex, suspended micro/nanoscale polymer fiber structures were generated with precise control, using the 3-axis dispensing system described above, via a custom automated direct-writing procedure. Specifically, prior to performing the direct-write procedure, three different complex fiber web structures: 1) a "quad" structure (Figure 3. 8a); 2) a "symmetric" structure (Figure 3. 9a); and, 3) a "dual chevron" structure (Figure 3. 10a), were designed in SolidWorks ${ }^{\mathrm{TM}}$ to define the precise spatial locations for the robot. These robot spatial instructions (Figure 3. 8b - Figure 3. 10b, respectively) were programmed into the JR-C controlling software [121]. Next, the completed fiber structure design program was compiled and transferred from the computer to the robot. Just prior to loading the polymer sample into the syringe, the robot stage offset was determined by repositioning the tip of the dispensing needle ( 25 gauge) from the default home position to the initiation point of the prescribed micro/sub-micron fiber structure to be drawn. A polymer solution sample of known concentration ( $24 \%$ by weight) was then loaded into the syringe barrel and flowed to the purging valve and needle. Subsequently, all the dispensing parameters for the valve controller and robot controlling software were set to the desired algorithm (Figure 3. 11). The prefabricated substrate was placed onto the robot stage platen located inside the plastic enclosure (Figure 3. 12). A USB microscope, mounted to the Z-axis and focused on the dispenser tip, was used to assist in precisely positioning the valve tip to the desired location on the prefabricated substrate or device. Upon completion of the direct-write process, all fibers' diameters created on the substrate were measured via a scanning electron microscope. 

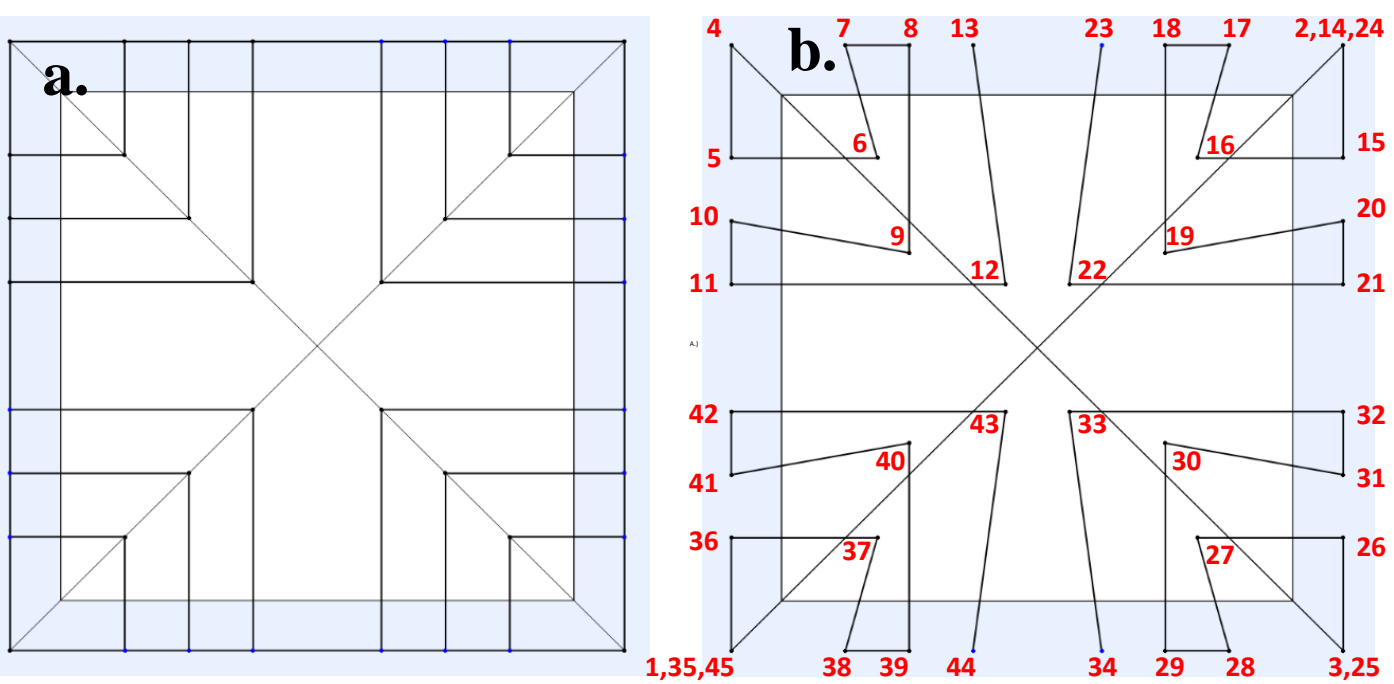

Figure 3.8 a.) Example of the design for the "Quad" web structure created in SolidWorks and, b) Sequential point-to-point spatial instructions inputted into the JR-C Point software.
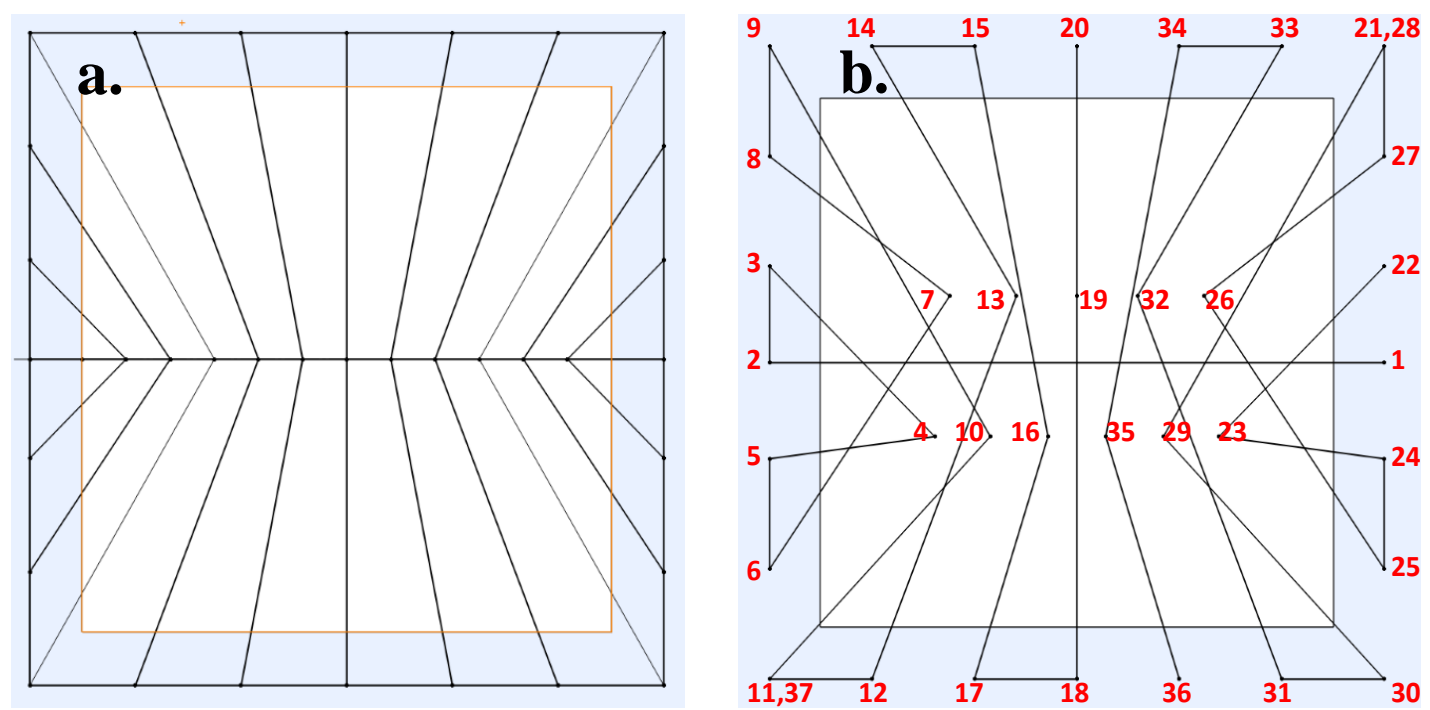

Figure 3.9 a) Example of the design for the "Symmetric" web structure created in SolidWorks and, b) Sequential point-to-point spatial instructions inputted into the JR-C Point software 

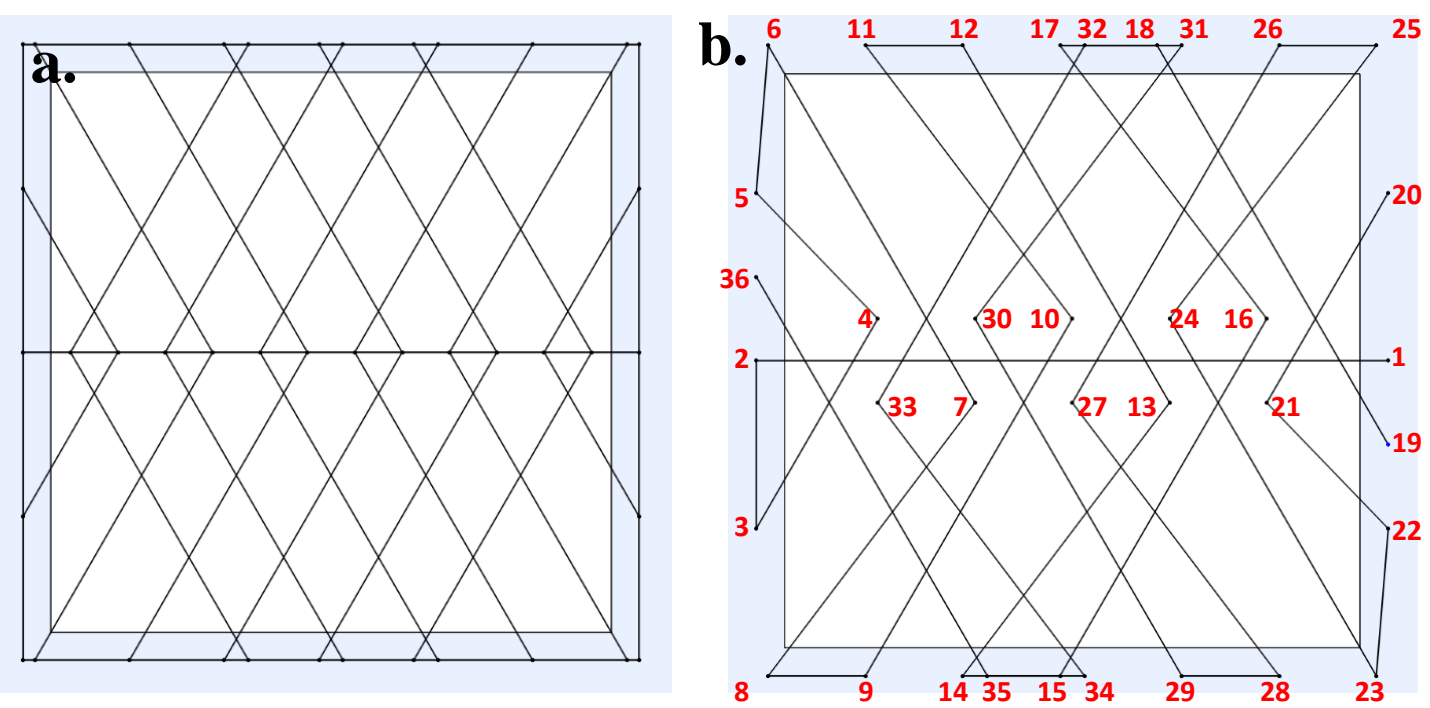

Figure 3. 10 a) Example of the design of the "Dual Chevron" web structure created in SolidWorks and, b) Sequential point-to-point spatial instructions inputted into the JR-C Point software

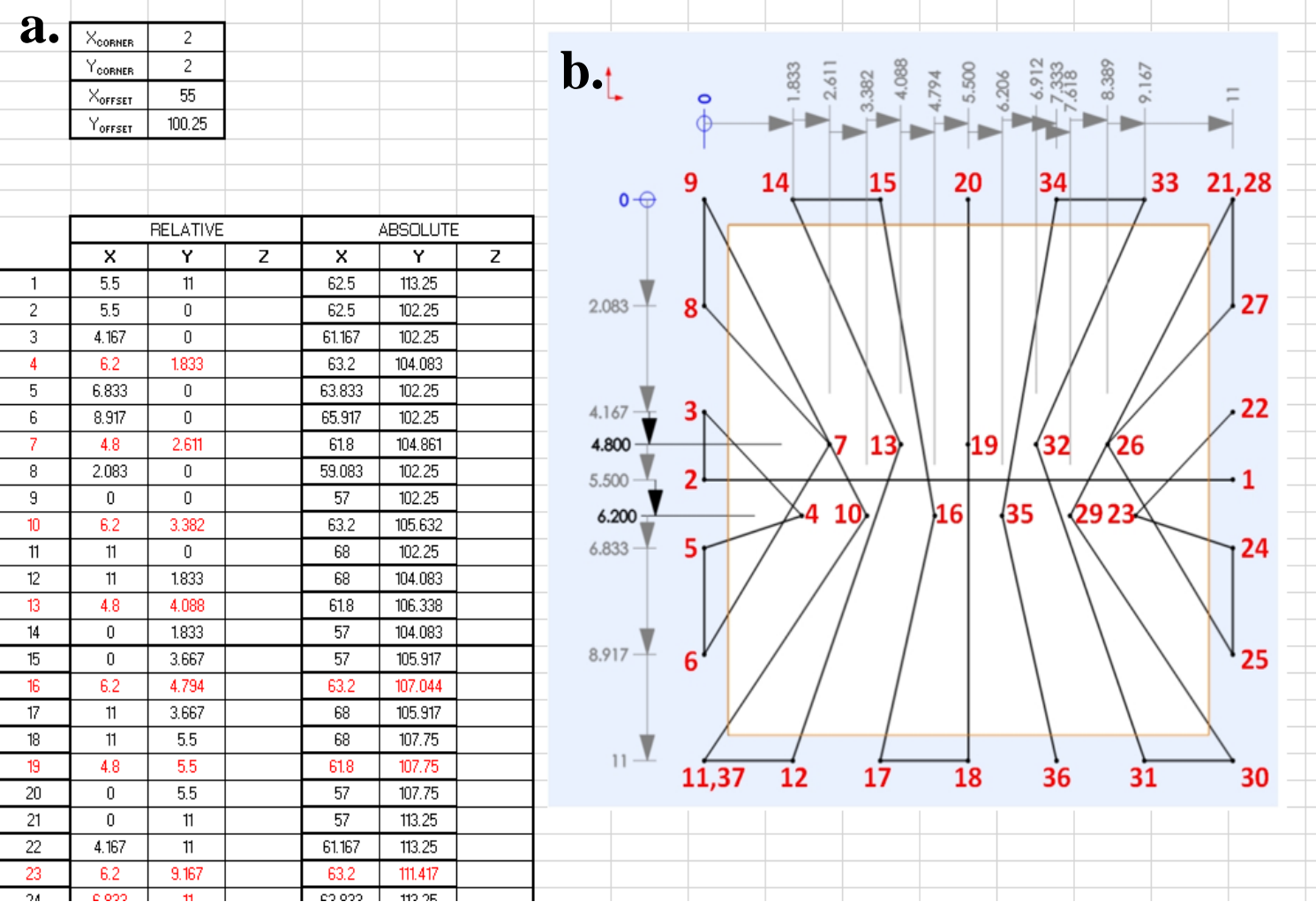

Figure 3. 11 Illustration of how the actual coordinates were determined for programming dispensing system: Left: Table of coordinates; Right: Graphical representation of coordinates on targeted substrate schematic 


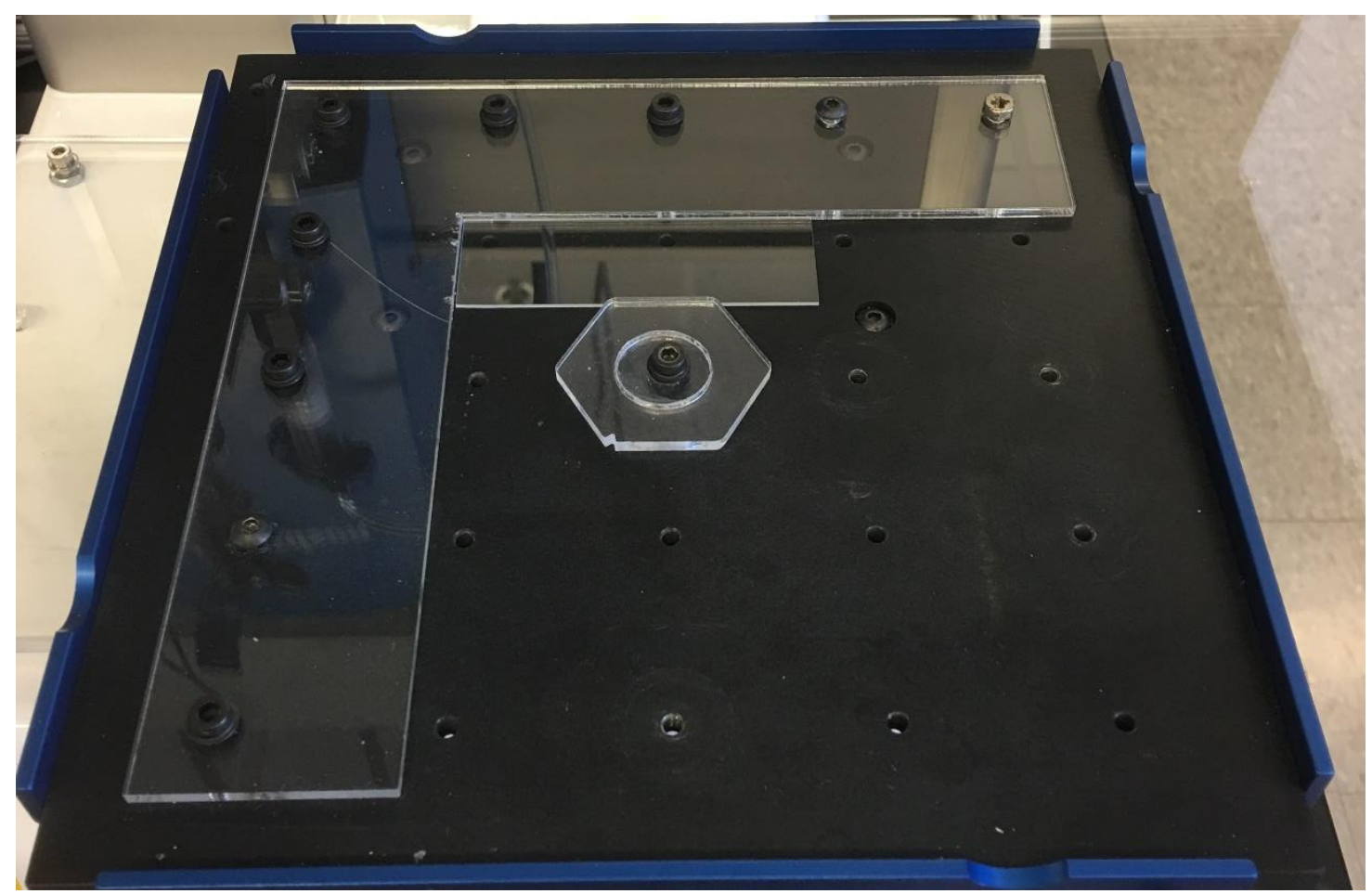

Figure 3. 12 Image of the robot stage platen with the substrate

\subsubsection{Characterization of Direct-write Process}

The purpose of the characterization process was to develop the methodology by which fibers of predefined diameter were repeatedly drawn through precise control of specific operating parameters and/or a combination thereof. Based on our previous work [119], PMMA solutions of $19 \%$ - 27\% (in 1\% increments) by weight and other operating parameters, needle tip sizes, feed rates, dispense time and fiber drawing lengths were initially selected to characterize the experimental process (Table 3. 1). Practical limitations of the dispensing system restricted the dispensing times; that is, when the dispensing time was shorter than $0.02 s$, the polymer solutions would not discharge from the needle tip, and $1 \mathrm{~s}$ was the longest dispensing time this system was capable of achieving. Here, a design of experiment (DOE) was utilized to assist in studying the system response output (fiber diameters) via purposely changing the input variables such as the feed rate, needle gauge, 
dispense time, fiber length, temperature, and polymer solution concentration. A total of 56,700 trials would have been needed for conducting a full factorial DOE; however, this was unrealistic since it would require a significant amount of time (on the order of years) to complete all of the experiments and fiber metrology measurements. Therefore, in this characterization procedure, only partial trials of all combinations of the operating parameters were executed to identify the critical factors that most greatly affect fiber diameter. A general linearized analysis of variance (ANOVA) model was performed in commercially-available statistical software (Minitab) to analyze the partial data [122]. As a result of these experiments, four factors including fiber length, needle size, polymer concentration, and dispense time were found to be the significant factors $(\mathrm{p}<0.05)$ influencing fiber diameter (Table 3. 2).

Table 3. 1 Direct-write process operating parameters used in the characterization experiments

\begin{tabular}{|c|c|}
\hline Controlling Parameters Types & Controlling Parameters Value \\
\hline Needle Tip Size (Gauge) & $25,27,30,32$ \\
\hline Feed Rates (mm/s) & $10,15,300-500(50 \mathrm{~mm} / \mathrm{s}$ \\
increments $)$
\end{tabular}


Table 3. 2 Critical direct-write process parameters identified in the characterization process

\begin{tabular}{|c|c|}
\hline Controlling Parameters Types & P Value \\
\hline Fiber Length $(\mathrm{L})$ & 0.000 \\
\hline Needle Size $\left(\mathrm{N}_{\text {gauge }}\right)$ & 0.000 \\
\hline Polymer Concentration $(\mathrm{C})$ & 0.000 \\
\hline Dispense Time $\left(\mathrm{T}_{\mathrm{D}}\right)$ & 0.001 \\
\hline
\end{tabular}

Using the output data, a preliminary prediction equation for determining the average fiber diameter based on the critical direct-write process parameters was generated as shown in equation 3-1:

$$
\mathrm{D}_{\text {avg }}{ }^{0.2240}=0.4815-0.0063 * \mathrm{~L}-0.0205 * \mathrm{~N}_{\text {gauge }}+8.2413 * \mathrm{C}+0.4451 * \mathrm{~T}_{\mathrm{D}}
$$

where $\mathrm{D}_{\text {avg }}$ was the predicted average diameter and $\mathrm{L}, \mathrm{N}_{\text {gauge }}, \mathrm{C}$ and $\mathrm{T}_{\mathrm{D}}$ are defined above in Table 3.2. While this model cannot predict the final fiber diameter with a high level of accuracy, a trend was capable of being extracted from the model, which guided additional process characterization in order to refine the model further. For example, additional experiments were performed to investigate the effect of environmental temperature on fiber yield. Specifically, to avoid fibers from breaking up at low polymer solution concentrations, the environmental temperature was controlled at $70^{\circ} \mathrm{F}, 80^{\circ} \mathrm{F}, 90^{\circ} \mathrm{F}$, and $100^{\circ} \mathrm{F}$, in order to increase the evaporation rate as well as viscosity to resist the drawing force during the writing process. As a result of all of these characterization experiments, it was determined that a needle gauge of 25 , feed rate of $15 \mathrm{~mm} / \mathrm{s}$, a dispense time of $0.02 \mathrm{~s}$ and an 
environmental temperature of $70^{\circ} \mathrm{F}$ were the best operating parameters to repeatedly and controllably fabricate sub-microscale 3-D oriented fibers.

Besides performing statistical analysis on the controlling experimental parameters, another model was implemented to investigate the effect of changing the physical properties of the polymer solutions on the fibers' diameter and "processability" of the fibers. Specifically, for each different concentration, three physical properties, including viscosity, surface tension, and solvent evaporation rate, were measured. Viscosity of all of these PMMA solutions were measured via cone-and-plate viscometer (e.g. LVDV-II+ and RVDV-II+, Brookfield Engineering Laboratories, Inc, Middleboro, MA) [123]. The fluid viscosity was calculated using the relationship between the measured torque, spindle multiplier constant and the speed of spindle:

$$
\eta_{\mathrm{s}}=T K \times S M C \times \frac{10000}{R P M}
$$

where $\eta_{s}$ was the viscosity, RPM was the speed of the spindle affixed to the cone, TK was the torque (0.09373 for LVDV-II+, 1 for RVDV-II+), and SMC was the spindle multiplier constant (9.83 for CP-52 - the Brookfield cone used in these studies). Additionally, the surface tension of the PMMA solutions were measured using the Wilhelmy technique, which involved placing the test solutions in a glass vial located on a high resolution balance ( scale resolution $=0.001 \mathrm{~g})$. A glass rod of known diameter was lowered towards the test solutions, where the position of the rod was controlled via a servo or stepper controlled linear actuator, until the rod just touched the surface and then partially immersed. In the meantime, a change in mass measurement was monitored and recorded on the balance when removing the rod from the solution's surface. Finally, surface tension was calculated 
using the force change in the actuator, perimeter of the rod, and contact angle of the fluid on the rod using the following formula:

$$
\sigma=\frac{F}{l \cos \theta}
$$

where $l$ was the wetted perimeter of $\operatorname{rod}(l=10.05 \mathrm{~mm}$ with a diameter $=3.2 \mathrm{~mm}), \theta$ was the contact angle of the fluid on the rod, $F$ was the change in force due to the surface tension $(\sigma)$ of the test fluid on the tip of the rod.

Lastly, the mass transfer coefficient of the polymer solutions were determined using thermogravimetric analysis (e.g. TGA 2950, TA Instruments, New Castle, DE) [123]. The general measurement process involved loading a $30 \mu \mathrm{L}$ sample of the polymer solution onto a platinum plate, prior to taring the balance for the sample. Subsequently, the TGA was programmed to run at the desired operating temperature $\left(70^{\circ} \mathrm{F}, 80^{\circ} \mathrm{F}, 90^{\circ} \mathrm{F}, 100^{\circ} \mathrm{F}\right)$ for 2 hours and the mass of target solution was monitored following venting of the chamber. Finally, the mass transfer coefficient $(\chi)$ was calculated using the measured changes in the solution's mass, area of solution/air interface and density:

$$
\chi=\frac{-m(t) m(t)}{A\left(m(t)-m_{P O L Y M E R}\right) \rho}
$$

where $\mathrm{m}(\mathrm{t})$ was the mass of PMMA solutions, A was the area of the solution/air interface (equals $78.5 \mathrm{~mm}^{2}$ for standard plate), $m_{P O L Y M E R}$ was the polymer mass in the solution and $\rho$ was the density of the solution.

With the physical and mechanical properties of polymer solutions determined, an empirical prediction equation was generated utilizing the previously collected characterization data together with performing a correlation analysis between the experimental parameters, initial conditions and the final fiber diameter. Our previous work 
indicated that PMMA behaved as a Newtonian fluid since $\frac{\sigma}{\eta}$ was linear; thus, the following empirical model was used and consisted of multiple parameters:

$$
D_{\infty}=f\left(P, C_{a}, \Lambda\right)
$$

where $\mathrm{P}$ was the dimensionless processability parameter $\left(P=\frac{\eta \chi}{\sigma}, \chi\right.$ was evaporation rate), $C_{a}$ was the capillary number $\left(C_{a}=\frac{\eta U}{\sigma}, \mathrm{U}\right.$ was feed rate), and $\Lambda$ was the aspect ratio $(\Lambda=$ $\left.\frac{L}{D_{0}}\right)$. While $C_{a}$ indicates the ratio of the drawing rate to the surface tension-driven thinning rate, the final diameter of the PMMA fibers was expressed as a function of three dimensionless parameters that relate to polymer droplet thinning mechanics $(P-$ the processability parameter), experiment control parameters ( $C_{a}-$ the capillary number), and polymer fiber dimensionality $(\Lambda-$ the aspect ratio). For the robotic dispensing system, the direct-write process was more controllable than the previous direct-write process developed by our group [119] due to the addition of two parameters, 1) pressurized dispensing flow; and, 2) dispense time. The dispense time was defined as the period the airflow was compressed onto the polymer solutions at the beginning of each fiber drawing procedure. Thus, in order to appropriately describe the direct-write process for the 3-axis robotic dispensing system, it was necessary to develop a new multi-parameter model (referred as to empirical prediction model) that included the previous dimensionless parameters together with the dispense time $\left(\mathrm{T}_{\mathrm{D}}\right)$ :

$$
D_{\infty}=f\left(P, C_{a}, \Lambda, T_{D}\right)
$$

A direct relationship existed between the controlling parameters and dimensionless parameters, which provided the variables of the empirical prediction equation. For instance, the capillary number was a function of viscosity, feed rate, and surface tension. One 
specific weight concentration of the PMMA polymer solution corresponded to only one specific value of viscosity and surface tension. The processability parameter was expressed as a function of viscosity, solvent mass transfer coefficient, and surface tension where the solvent mass transfer coefficient was constant due to only one solvent being used in this study. The aspect ratio was defined by fiber length and the initial diameter, which was defined by the inner diameter of the syringe needle tip.

Exploiting the polymer solutions' intrinsic and physical properties together with the robotic system's operating parameters, an empirical predictive equation was obtained in Matlab using a generic algorithm to find the minimum fiber diameter that can be fabricated based on the corresponding variables (or parameters) of the equation. This enabled the determination of the optimal combination of all controlling factors to produce specific, prescribed fiber diameters. Subsequently, experiments were conducted to validate this algorithm and statistical analysis using ANOVA was performed to determine the error of the equation's predictive value.

\subsubsection{Measurement of PMMA Fiber Diameters}

Upon completion of the direct-write process, the fibers were sputter coated with gold-palladium alloy for 2 to 3 minutes to deposit a $10 \mathrm{~nm}$ thick metal layer to avoid charging during the SEM (Zeiss Supra 35VP, Thornwood, NY; high voltage set at $\sim 2.00$ $5.00 \mathrm{KV}$ ) imaging process [124]. Each fiber was imaged three times at points $200 \mu \mathrm{m}$ away from the initiating and terminating position plus the middle location to ensure fiber diameter uniformity. 


\subsection{Micro/Nanofluidic Platform Fabrication}

\subsubsection{Overview of the Fabrication Process}

In general, the overall process flow for fabricating the micro/nanofluidic platform consisted of nine key processing steps (Figure 3. 13). First, a mold with two microchambers on glass substrate was created using dry film photolithography and a prescribed micro/nanoscale fiber was drawn across the micro-chamber mold with the robotic dispensing system. Another mold with the same structure as the first one was fabricated on a flexible Delrin substrate using a similar method. Next, the glass mold was placed on the hotplate, then flipped and placed over the flexible substrate mold, followed by alignment of these two structures under a high precision stereomicroscope and bonding. The flexible substrate was not peeled off from the assembly on the top of the glass substrate until the two structures were firmly bonded and adhered together on a hotplate at an elevated temperature $\left(100{ }^{\circ} \mathrm{C}\right)$. PDMS was poured on the mold containing the two layer microstructures sandwiching a nanofiber or microfiber and then transferred to an oven at $60{ }^{\circ} \mathrm{C}$ overnight to assist in the solidification of the PDMS casting. The PDMS was peeled from the glass substrate. As the last step in the PDMS preparation process, the buried PMMA fiber was solvated by placing the PDMS substrate in a beaker filled with acetone and sonicating the beaker for 30 minutes. 


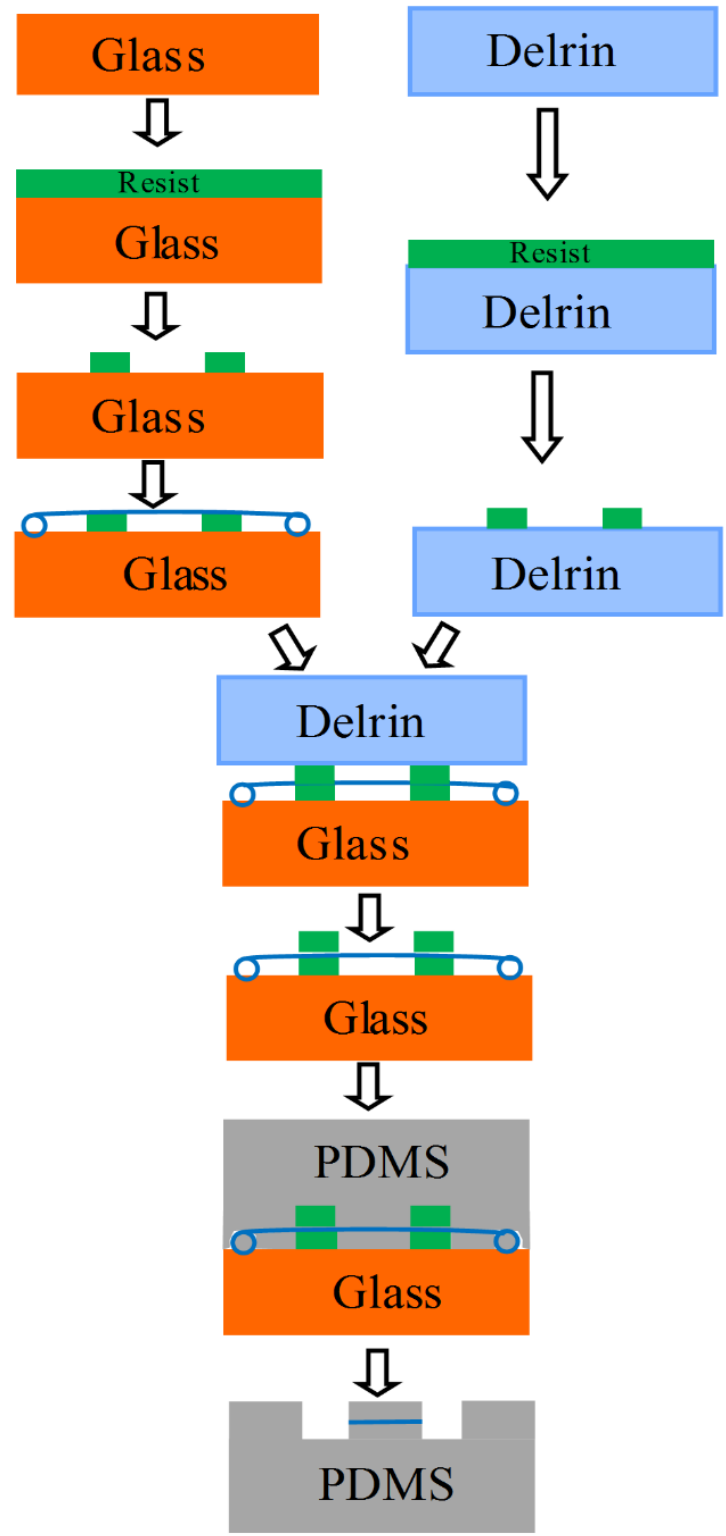

Figure 3. 13 Schematic of the process flow for fabricating the electroporation devices

\subsubsection{Dry Film Photolithography}

A photomask consisting of the three juxtaposed, tip-to-tip micro chamber convex structures were designed in L-Edit and fabricated via a Heidelberg DWL 66fs Laser Writer (Heidelberg Instruments Mikrotechnik GmbH, Germany) in combination with traditional 
photolithography techniques (including direct writing, development, and chrome etching). The glass substrates were first prepared using organic solvents to clean the surface of the substrates. Subsequently, the negative contrast dry film resists (DFRs, $t=15 \mu m$, Ordyl SY 320, ElgaEurope, Milan, Italy, a solvent type permanent dry film for special MEMS applications) was cut to match the size of the substrate. The DFRs were selected over traditional liquid resists due to superior planarity and excellent adhesion to different substrates including, but not limited to, glass, $\mathrm{Si}$, and Delrin as previously described by others [125-127]. The easy-to-remove protection layer of the dry film resist was removed with a razor blade and the resist was transferred onto the substrate using a dry film laminator. The standard operating procedure on how to use the dry film laminator was presented in detail in Appendix A. The resist and substrate were placed on an $85^{\circ} \mathrm{C}$ hotplate and allowed to bake for 2 minutes. Once the substrate cooled down, it was transferred to a custom collimated UV exposure system, consisting of a 10W $365 \mathrm{~nm}$ LED (Figure 3. 14). The photomask was placed on the top of the resist-coated substrate and exposed to the 365 $n m$ UV light source for 4 minutes. After the exposure was complete, the substrate was treated with a post-exposure-bake for 2 minutes at $85^{\circ} \mathrm{C}$ on a hotplate. Once the substrate cooled down to room temperature, the top protection layer of the resist film was peeled from the substrate for development. The substrate was developed in a 10:1 volume ratio of Xylene to IPA for 1 minute to solvate away the unexposed structures.

Characterizing the dry film resist photolithography process included: 1) optimizing exposure time, post-exposure-bake time and temperature, and development time; 2) developing the dry film resist transferring process to minimize bubble formation during adhesion to the substrates; and, 3) identifying the most appropriate development solutions 
for completely removing the resist's residue after development. Optimization of these experimental parameters (Table 3. 3, Table 3. 4) enabled an easier fabrication process to be employed, better vertically thick structures (as thick as $15 \mu \mathrm{m}$ ) to be created, a low cost process to be implemented, and excellent adhesion to a variety substrates compared to the liquid resists.

Table 3. 3 Optimized parameters for the DFRs photolithography processes on a glass substrate

\begin{tabular}{|c|c|}
\hline Dry Film Laminator & Speed 5 at $200^{\circ} \mathrm{F}$ \\
\hline Soft bake time & $1 \mathrm{~min}$ \\
\hline Exposure time & 4 mins \\
\hline Post exposure bake time & 2 mins \\
\hline Post bake temperature & $85^{\circ} \mathrm{C}$ \\
\hline Development solutions & $\begin{array}{c}\text { Xylene and IPA (10:1 } \\
\text { volume ratio })\end{array}$ \\
\hline Development times & 1 min \\
\hline
\end{tabular}

A number of different soft substrate materials (paper, white delrin, acrylic, rubber, plastic, etc.) were utilized to characterize the adhesion process in order to align and bond the soft substrates with the rigid glass substrate. Black Delrin demonstrated to be the best soft substrate material with superior adhesion to the DFRs and better tolerance of the development solutions compared to the other soft substrate materials tested. The fabrication process for the Delrin substrate varied slightly from the glass or silicon wafer substrate processing during the thin-film coating process. Before transferring the thin-film 
layer onto the Delrin substrate, the Delrin substrate required a flame plasma treatment step to increase the adhesion of the resist.

Table 3. 4 Optimized parameters for the DFRs photolithography processes on a Delrin substrate

\begin{tabular}{|c|c|}
\hline Plasma treatment time & $1 \mathrm{~min}$ \\
\hline Dry Film Laminator & Speed 5 at $200{ }^{\circ} \mathrm{F}$ \\
\hline Soft bake time & $1 \mathrm{~min}$ \\
\hline Exposure time & 5 mins \\
\hline Post exposure bake time & 2 mins \\
\hline Post bake temperature & $85^{\circ} \mathrm{C}$ \\
\hline Development solutions & $\begin{array}{c}\text { Xylene and IPA (10:1 } \\
\text { volume ratio) }\end{array}$ \\
\hline Development times & 1 min \\
\hline
\end{tabular}




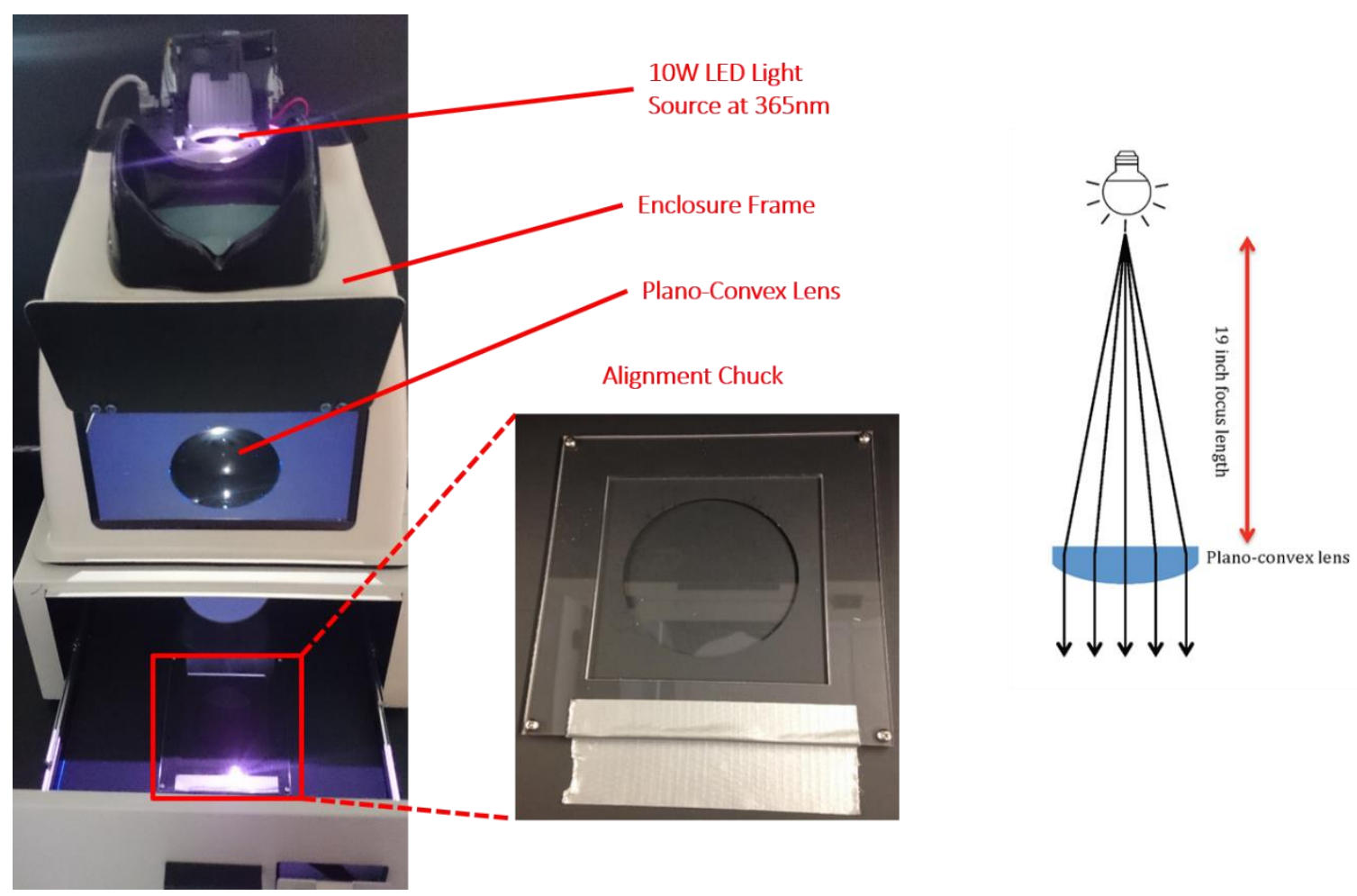

Figure 3. 14 Image of the custom collimated UV exposure system

\subsubsection{Drawing Fibers onto Dry Film Structure}

A prescribed PMMA microfiber or nanofiber was drawn via the robotic dispensing system described above. The initiation position for the fibers was located across the center tips of the two micro-chambers. Prior to the direct-writing step, the desired polymer solutions were loaded into the pressurized barrel of the 3-axis robotic dispensing system, as described in section 3.2.1. The fibers were written onto the glass substrate coated with a single layer of DFRs using the following procedures: 1) designed the desired fiber structure pattern using a CAD software package (SolidWorks) and inputted the corresponding spatial coordinates $(\mathrm{x}, \mathrm{y}, \mathrm{z})$ into the robot JR-C controlling software for all initiation and termination points; 2) transferred the completed fiber structure design program from the computer to the robot by clicking "Send C\&T Data" under the Robot 
menu within the JR-C software; 3) determined the robot stage offset to reposition the tip of the dispensing needle from the default "home" position to the initiation point of the prescribed fiber structure to be drawn on the DFR patterned glass substrate. A USB microscope $($ magnification $=200 \mathrm{X})$ mounted to the dispensing system valve bracket in order to translate along the $\mathrm{Z}$-axis was manually focused on the dispenser tip to assist in precisely positioning the valve tip to the desired location; 4) loaded $3 \mathrm{ml}$ of the PMMA solution sample of known concentration into the syringe barrel, purged the valve and needle, and set all the dispensing parameters for the valve controller and robot controlling software; 5) placed the prefabricated substrate with two micro chamber structures onto the robot stage platen, closed the thermal enclosure doors and wrote the fibers onto the substrate by clicking the robot menu from JR-C software and selecting "test running"; 6) DC sputter coated a conductive gold/platinum/alloy metal layer for 2 min until a $2 \mathrm{~nm}$ thick layer of gold was deposited around the drawn fibers to allow visualization of the fibers in the scanning electron microscope; 7) measured the diameter and structure of the fibers via scanning electron microscope (SEM). SEM parameters: High voltage level - $2.00 \mathrm{kV}$; Objective - InLens; Working Distance - $9.0 \mathrm{~mm}$. The last process consisted of cleaning all the dispensing system parts (as described in the last paragraph of section 3.2.1) after disassembling the system in order to perform next experiment.

\subsubsection{Film-to-Film Alignment and Bonding}

A custom-made alignment and bonding system was fabricated consisting of a stereoscope and a 3-axis stage (Figure 3. 15). The high magnification stereoscope provided the magnification required to align the two microscale film structures, while the 3-axis stage was utilized to move one substrate directly overtop of the other substrate to the exact 
desired position for aligning the two microchamber tips. In addition, the resistance heater located underneath the heater plate heated the two substrates to the ideal bonding temperature after proper alignment was achieved. The Delrin substrate was placed on the aligner loading chuck, which was movable in the $\mathrm{X}, \mathrm{Y}$, and $\mathrm{Z}$ directions and also rotated along $\mathrm{Z}$ axis. A thin, square aluminum plate, which contained a hole for applying vacuum to the substrate to hold it in place, was used as the heater plate to complete the bonding system (Figure 3. 16). A silicone rubber gasket was laser-cut with two slots to enable the two rectangular resistance heaters to be slid under the aluminum plate. The silicone rubber was slightly thicker than the resistance heater to reduce the amount of pressure experienced by the two heaters when a load was applied to the two stages during the bonding process. For the upper loading plate (Figure 3. 17), a well was machined in the aluminum for placement of the glass substrate. Additionally, two holes were drilled in the metal plate: 1) the big hole was utilized to observe small features on the glass and Delrin substrates using the stereomicroscope; and, 2) the small hole connected to the vacuum line to apply a vacuum onto the glass substrate. Then, a small rectangular trench was machined in the bottom of the well to distribute the vacuum load over a larger surface area. Four holes were tapped into the upper loading plate to affix the plate onto the 3-axis immovable stage with four screws and washers.

General alignment and bonding consisted of eight main steps. First, a Delrin mold was placed on the aligner loading chuck just covering the vacuum hole. Then, a glass mold was placed on the upper loading plate. Subsequently, the vacuum was turned on to draw these two molds onto the upper and bottom loading chucks, respectively. The upper loading plate with the glass substrate was screwed tightly to the 3 -axis stage and the heater was 




Figure 3. 15 Image of the custom-made film-to-film alignment and bonding system turned on to let the thin film resists accumulate enough heat and to bond. Next, two small features on two molds were aligned using the stereomicroscope. In this step, it was necessary to align them with low magnification first and then gradually increase the magnification to achieve a much higher precision during the alignment process. This step was critical, so much care was taken since this was an irreversible alignment process and 


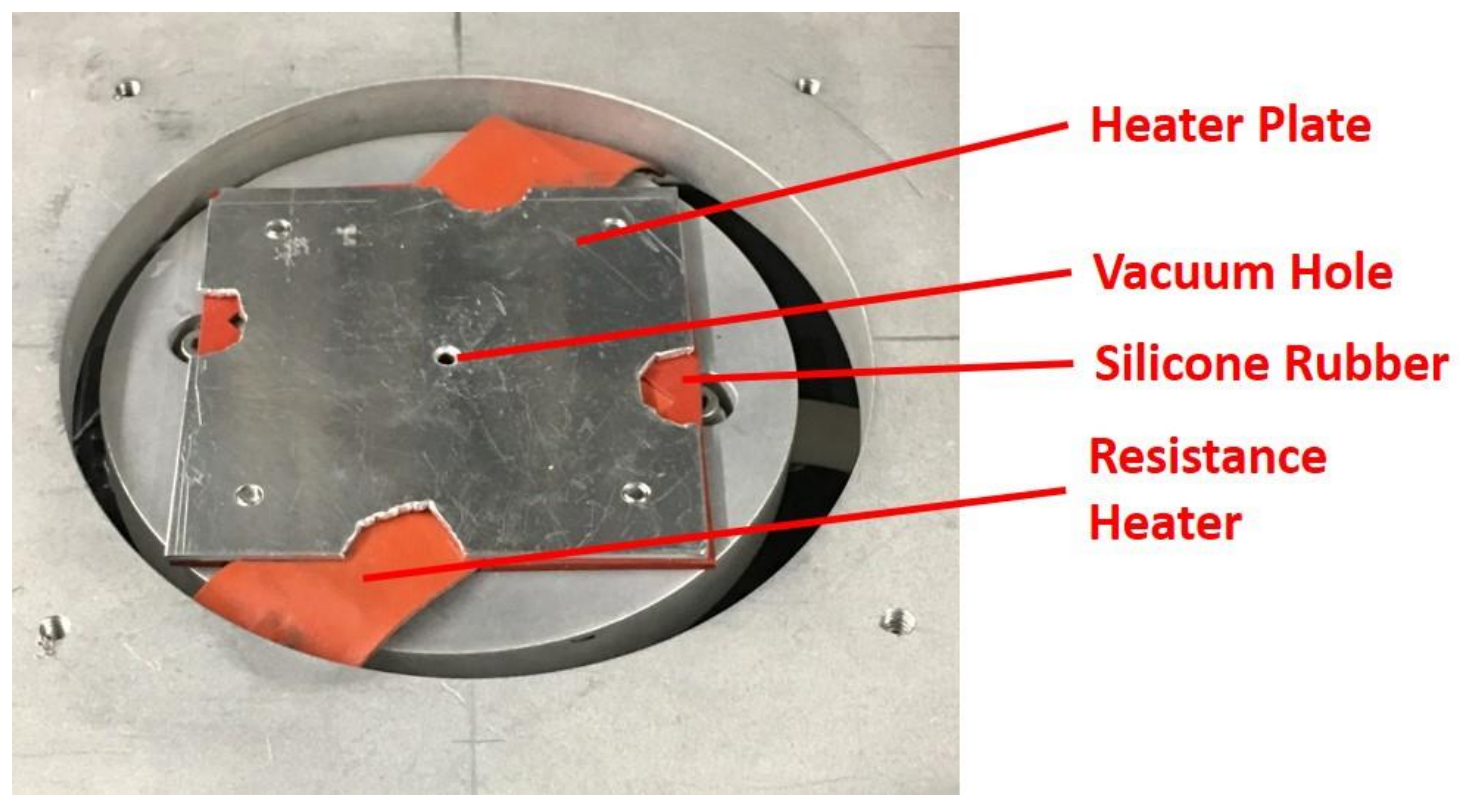

Figure 3. 16 Image of the aligner loading chuck of the custom-made film-to-film alignment and bonding system

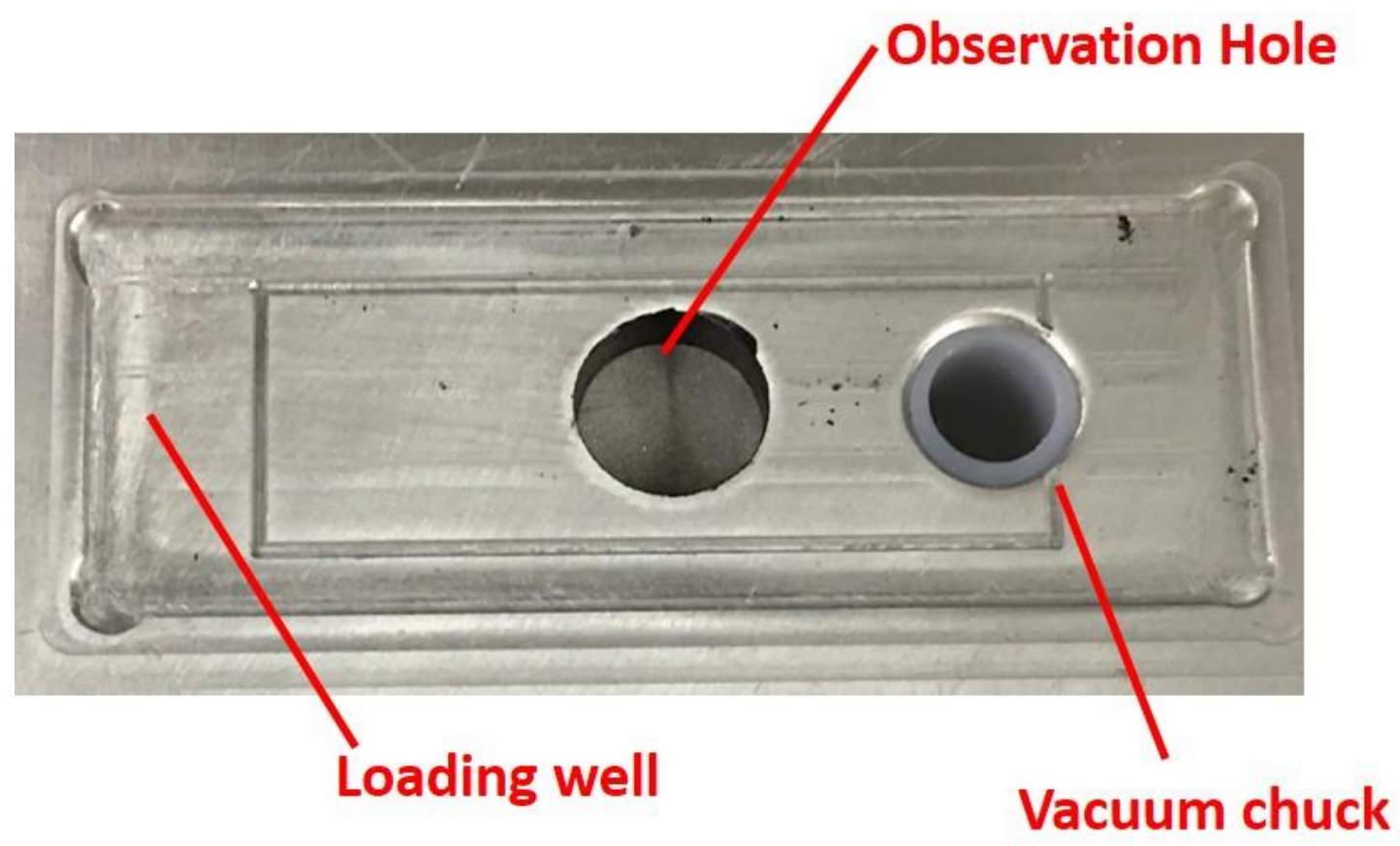

Figure 3. 17 Image of the upper loading plate of the custom-made film-to-film alignment and bonding system 
the micro/nanoscale fiber lying between the two molds broke or relocated with any minor mistake. After the two small features overlapped, the aligner loading chuck was lifted up to apply sufficient pressure onto the two films to bond them together at an elevated temperature $\left(100^{\circ} \mathrm{C}\right)$. Compared to other alignment and bonding systems, this equipment had several advantages including: 1$)$ higher yield ( 95\%); 2$)$ higher precision alignment (within $\sim 2 \mu \mathrm{m}$ tolerance); 3) feasible for any flexible or rigid substrate; and, 4) ease to operate.

\subsubsection{Replica Molding}

Once the alignment and boding process was completed, the mold was placed in a petri dish for replica molding. First, polydimethylsiloxane (PDMS) and its curing agent (1:10 weight ratio of the curing agent to PDMS) were mixed with a stirring bar for 2-3 minutes. Subsequently, the PDMS mixture was poured slowly into the petri dish to cover the substrate mold, taking great care to avoid breaking the suspended micro/nanoscale polymer fibers (Figure 3. 18). Then, the petri dish containing the mold and PDMS mixture was placed in a vacuum chamber for 30 minutes to remove air bubbles trapped in the PDMS mixture solution. Next, this petri dish was transferred to an oven and cured at $60^{\circ} \mathrm{C}$ overnight. 


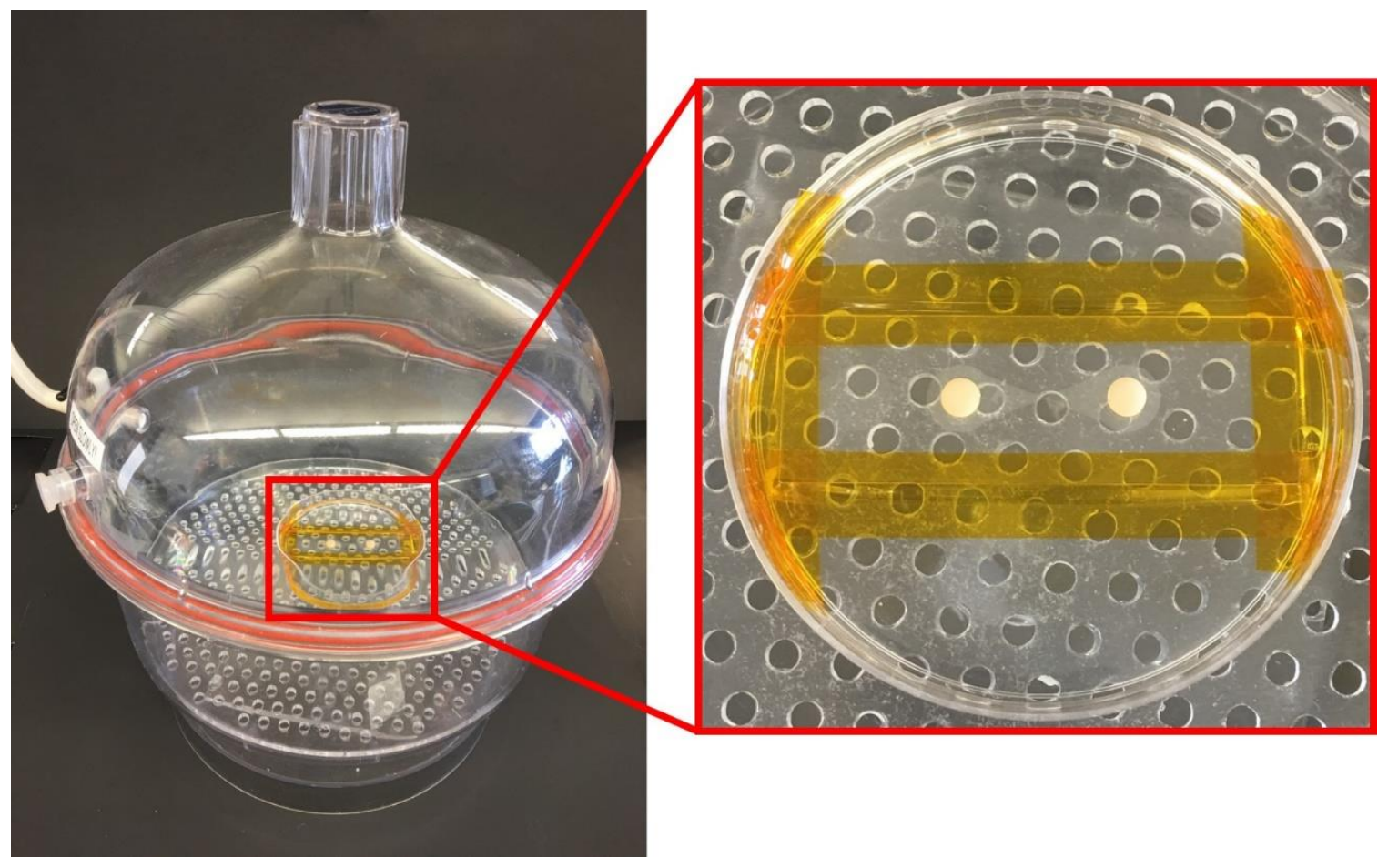

Figure 3. 18 Images of the substrate mold covered with PDMS (right) in the vacuum chamber (left)

\subsubsection{Nano/Micro Polymer Fibers Removal}

After curing overnight, the PDMS was peeled off from the glass substrate and the embedded polymer fibers were removed by placing the PDMS substrate in an ultrasonic acetone bath for 30 minutes (Figure 3. 19). Due to the considerably high swelling rate of the PDMS in acetone, the sonication time was carefully controlled to minimize the amount of swelling in order to maintain the integrity of the nanochannel. Afterwards, the PDMS replica mold was exposed to oxygen plasma to make the two micro-chambers' and micro/nanochannel's surfaces hydrophilic and enhance adhesion of the PDMS to the glass substrate. Subsequently, two inlet holes with a $5 \mathrm{~mm}$ center-to-center distance were 


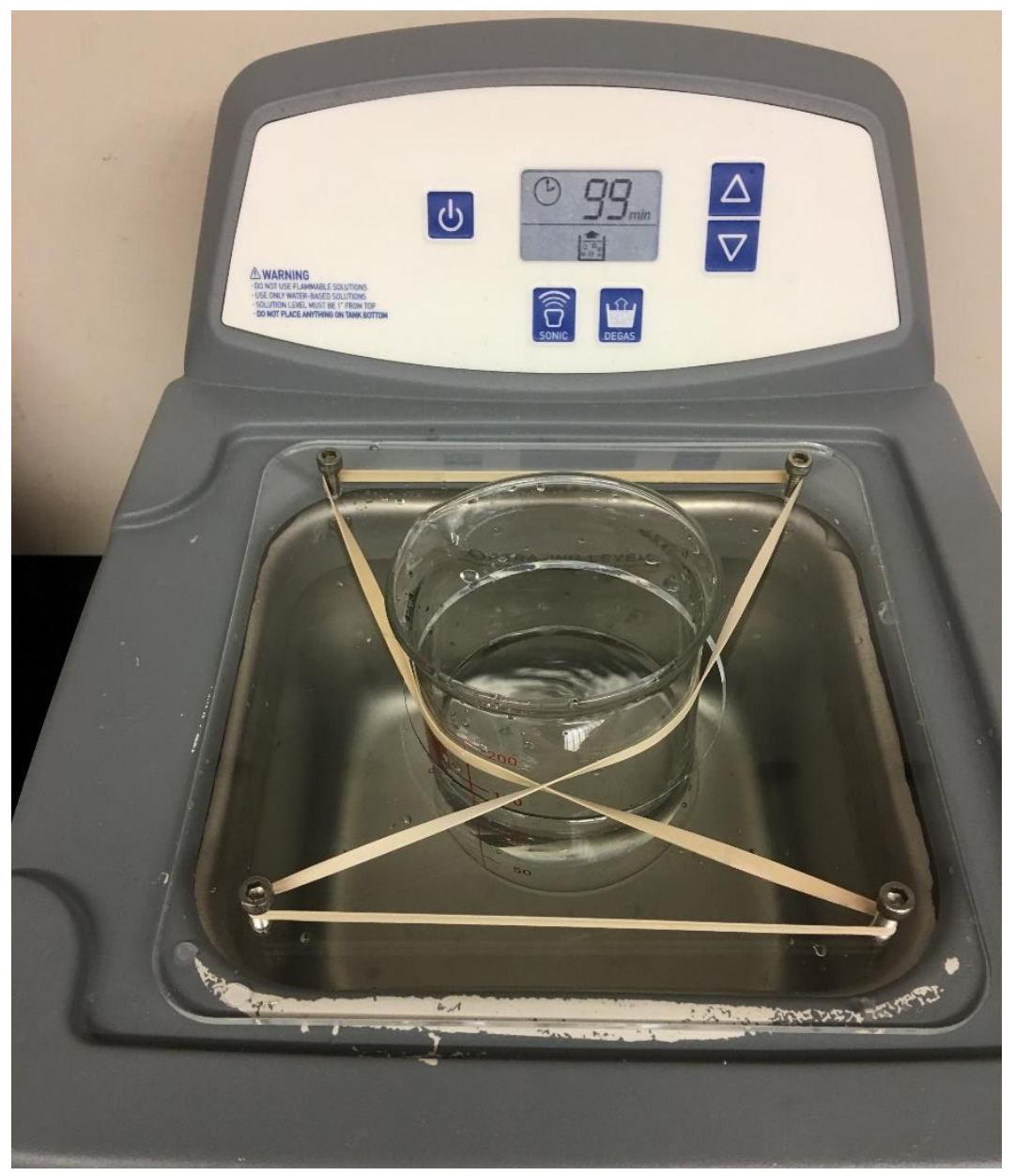

Figure 3. 19 PDMS Substrate in an Ultrasonic Acetone Bath

punched through the PDMS using a hole puncher. For storage, the samples were submerged in methanol solutions or DI water to maintain their hydrophilic properties until the start of the application experiments.

\subsection{Testing and Characterization}

In this study, each platform was characterized to determine the appropriate parameters for loading and transporting molecules through the micro/nanochannels. After each platform was tested for molecule transport, HL-60 cells (Human promyelocytic leukemia cells) were loaded into the platforms, translated to the micro/nanochannel via 
laser trapping and electroporation experiments performed. Finally, the images were analyzed to determine the time course for the cell electroporation process.

\subsubsection{Platform for Transportation of Molecules}

Before the cell electroporation experiments, the fabricated electroporation platforms were evaluated to confirm the existence of a micro/nanochannel and its connectivity to the two micro-chambers. Since the micro/nanochannel was buried inside the bulk device, it was difficult to acquire high quality images via SEM or high precision optical microscopy due to the distance from the channel to the top surface of the PDMS device. This distance resulted in low quality images due to the inability to accurately focus onto the micro/nanochannel and lack of contrast between the air filled channel and the PDMS material itself. Thus, two methods were used to determine channel patency: 1) electrical resistance measurements; and, 2) fluorescent dye method.

For the electrical resistance measurements, phosphate buffer solutions (PBS) with known conductivity were injected into each of the two micro-chambers through the two 5 $m m$ inlets using a $1 \mathrm{~mL}$ syringe. Next, the resistance of the micro/nanochannels filled with PBS was determined by inserting a platinum electrode $(\mathrm{d}=0.5 \mathrm{~mm})$ in each inlet, applying a voltage $(3 \mathrm{~V}$ to $12 \mathrm{~V})$ to the micro-chambers and measuring the current between the two micro-chambers with a power source meter. Finally, the experimental resistance value of each specific micro/nanochannel was compared to the theoretical value (based on the dimension of the micro/nanochannel and conductivity of the PBS) to confirm the micro/nanochannel's patency and its ability to connect the two micro-chambers.

The second technique performed to demonstrate patency of the channel involved imaging fluorescent particles (Propidium Iodide, Exitation/Emission $533 \mathrm{~nm} / 567 \mathrm{~nm}$, 
Thermo Fisher Scientific, Waltham, MA) using a confocal microscope (Nikon Eclipse Ti, Nikon, Minato, Tokyo, Japan). For these experiments, one micro-chamber was filled with regular PBS and the other micro-chamber was filled with the fluorescent nanoparticles suspended in PBS $(100 \mu \mathrm{g} / \mathrm{mL})$. Once again, the platinum electrodes were inserted into the two inlets of the PDMS substrate. The electrode located in the micro-chamber with the PI dye was connected to the cathode of the power source meter and the electrode located in the pure PBS loaded micro-chamber was connected to the anode. When the voltage (6 V to $12 \mathrm{~V}$ ) was applied across the two micro-chambers, the fluorescent nanoparticles migrated from the PI dye filled micro-chamber across the micro/nanochannel to the PBS filled chamber. Under the confocal microscope, the fluorescent nanoparticles were observed and their trajectory was captured and imaged using the software packages associated with the confocal imaging or video capture mode system. If the channels were occluded, only dark/black images were obtained; however, when the channels were patent, red particles were observed traversing the two micro-chambers through the micro/nanochannels.

\subsubsection{Platform for Electroporation in Cells}

Once each electroporation platform was verified as being functional, cells were loaded into one micro-chamber and one cell was trapped at the interface of the micro/nanochannel and the micro-chamber tip using an optical laser. Next, biomolecules were loaded into the other micro-chamber, and a voltage, of appropriate amplitude and duration pulse, was applied across the two sides of the micro-chambers to transfer the biomolecules into the living cell. By carefully controlling the dimension of the nanochannel together with the amplitude and duration of the applied electrical field pulse, a precise 
amount of biomolecules were allowed to enter the living cell with low cell mortality. Further details on each of these steps have been described below.

\subsubsection{Cell Culture}

HL-60 (Human promyelocytic leukemia) cells were obtained from Cascade Biologics and cultured in RPMI (Roswell Park Memorial Institute) 1640 medium with 10\% FBS (Fetal Bovine Serum) and 1\% PS (Penicillin Streptomycin). These cells were incubated at $37{ }^{\circ} \mathrm{C}$, under humidified atmosphere at $5 \% \mathrm{CO}_{2}$. The basic process for culturing the HL- 60 cells consisted of the following steps: 1) removed half the amount of media from the flask/plate; 2) rinsed the flask with half the amount of fresh media; 3) spun down the suspension (around $5 \mathrm{~mL}$ ) at $2000 \mathrm{rpm}$ for five minutes; 4) removed the supernatant of the pellet; 5) re-suspended the pellet in a volume that was easily aliquoted equally to the flasks/plates; 6) topped off with fresh media to a normal level $(\sim 10 \mathrm{~mL})$ in the flasks; and, 7) placed the flasks/plates back in the incubator. The cells were then stored in liquid nitrogen for future usage by: 1) preparing a final solution containing the complete media with $10 \%$ volume-to-volume serum and 10\% volume-to-volume Dimethyl Sulfoxide (DMSO); 2) repeating steps 1-7 above, then transferred $0.8 \mathrm{~mL}$ into a labeled vial; 3) adding $0.1 \mathrm{~mL}$ serum solution and $0.1 \mathrm{~mL}$ DMSO into the vial; 4) placing the vial into a cryogenic box that was placed into a $-80{ }^{\circ} \mathrm{C}$ freezer for at least 24 hours; and, 5) transferring the box to liquid nitrogen.

Prior to running the cell electroporation experiments, the HL-60 cells were removed from the liquid nitrogen using the following steps: 1) thawed the vial by gentle agitation in a $37^{\circ} \mathrm{C}$ water bath, keeping the O-ring and cap out of the water to reduce the possibility of contamination; 2) removed the vial from the water bath after $\sim 2$ minutes making sure the 
ice contents were completely thawed, then dipped or sprayed the vial with $70 \%$ ethanol to avoid contamination; 3) transferred cell solutions to a centrifuge tube containing $4 \mathrm{~mL}$ of fresh culture media and spun for 5 minutes at $2000 \mathrm{rpm}$; 4) re-suspended the pellet in a volume that was easily aliquoted equally to the flasks/plates; 5) topped off with fresh media to normal level ( $10 \mathrm{~mL})$ in flasks; and, 6) placed the flasks/plates back in the incubator.

\subsubsection{Optical Laser Trap Cells}

The HL-60 cells were trapped and manipulated using a laser trapping technique where a laser beam was focused with high precision to create a momentum force that was transferred to the cells when the cells were illuminated by the laser beam [128]. Movement of the laser beam and/or changing the beam focus enabled translation of the cells in all three spatial directions $(\mathrm{x}, \mathrm{y}$, and $\mathrm{z})$. It has been reported that particles ranging in diameter from a few Angstroms up to $10 \mu \mathrm{m}$ can be trapped with forces within a few hundred $\mathrm{pN}$ [128]. To minimize the risk of damaging the cells, the optical trapping performed in this study used near-IR lasers in the range of $800 \sim 1100 \mathrm{~nm}$ [129]. The trapping time was carefully controlled to ensure that the laser energy was not absorbed by the PBS, which could result in heating of the nanoscale volumes [128]. Heating had to be avoided in order to prevent damage to the cells, especially if the handling time of the cells was long. To laser trap the cells to the desired location in a 3-D micro/nanoscale fluidic device, a $\mathrm{Ti}$ series inverted microscope (Figure 3. 20) with laser excitation sources (Nikon Eclipse TIFL) and an objective lens (Nikon 60× A/1.20 WI, WD 0.31-0.28, Figure 3. 21) was used to image and capture the cell during the trapping process. This system consisted of TI-FL Epi-fl illuminator main body, a laser source $(980 \mathrm{~nm})$, a USB 2.0 digital camera (Thorlabs), a laser diode controller (Thorlabs LDC 220 C, Figure 3. 22), and a temperature controller 
(Thorlabs TED 200). The USB 2.0 digital camera was used to image or capture the cells during the trapping process. The laser diode controller enabled careful regulation of the power of the laser to trap and translate cells without damaging the cells. If the laser intensity was too high and exceeded the limit of the threshold temperature, the laser temperature controller set off an alarm.

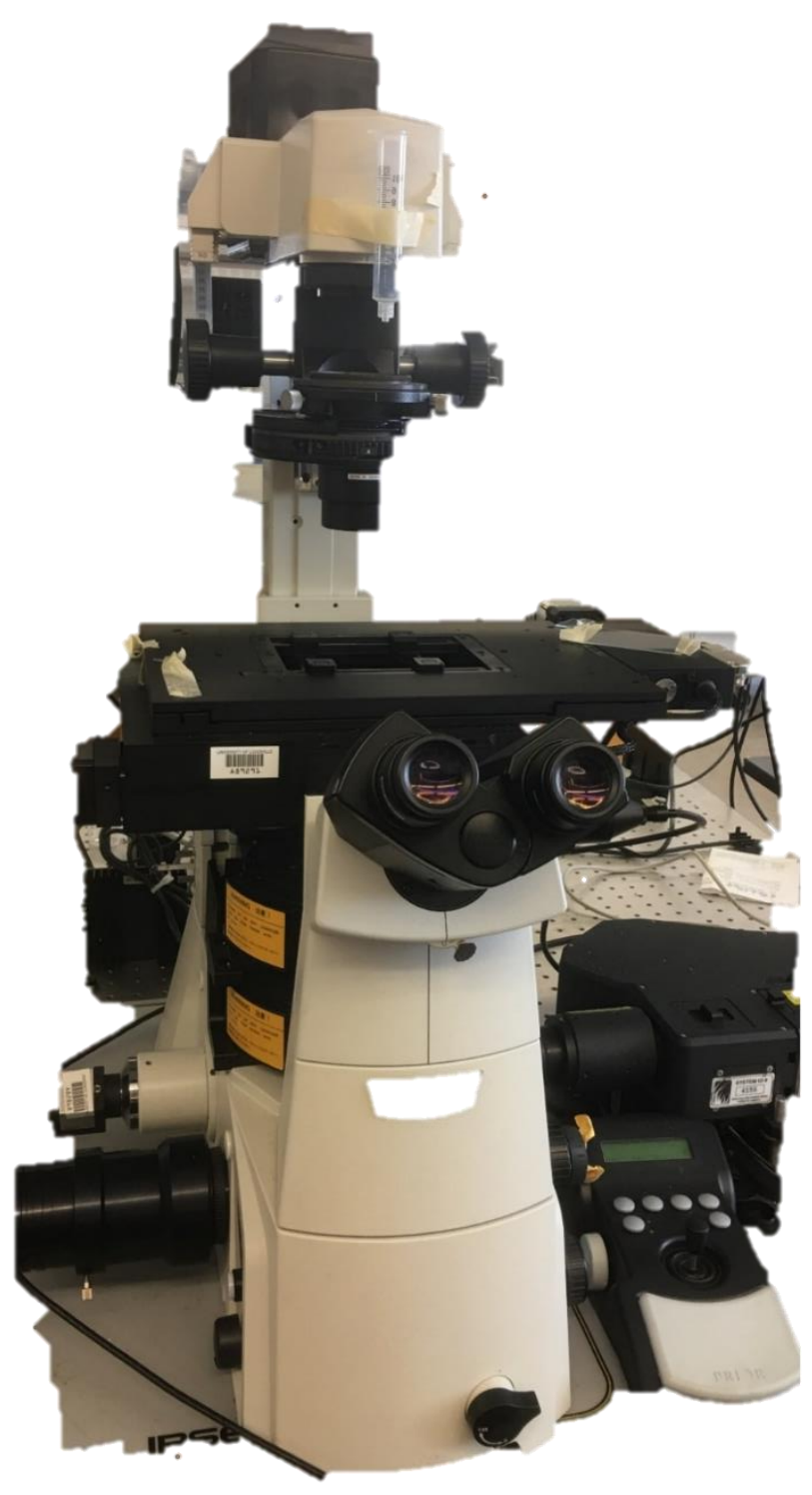

Figure 3. 20 Image of the Ti series inverted microscope 


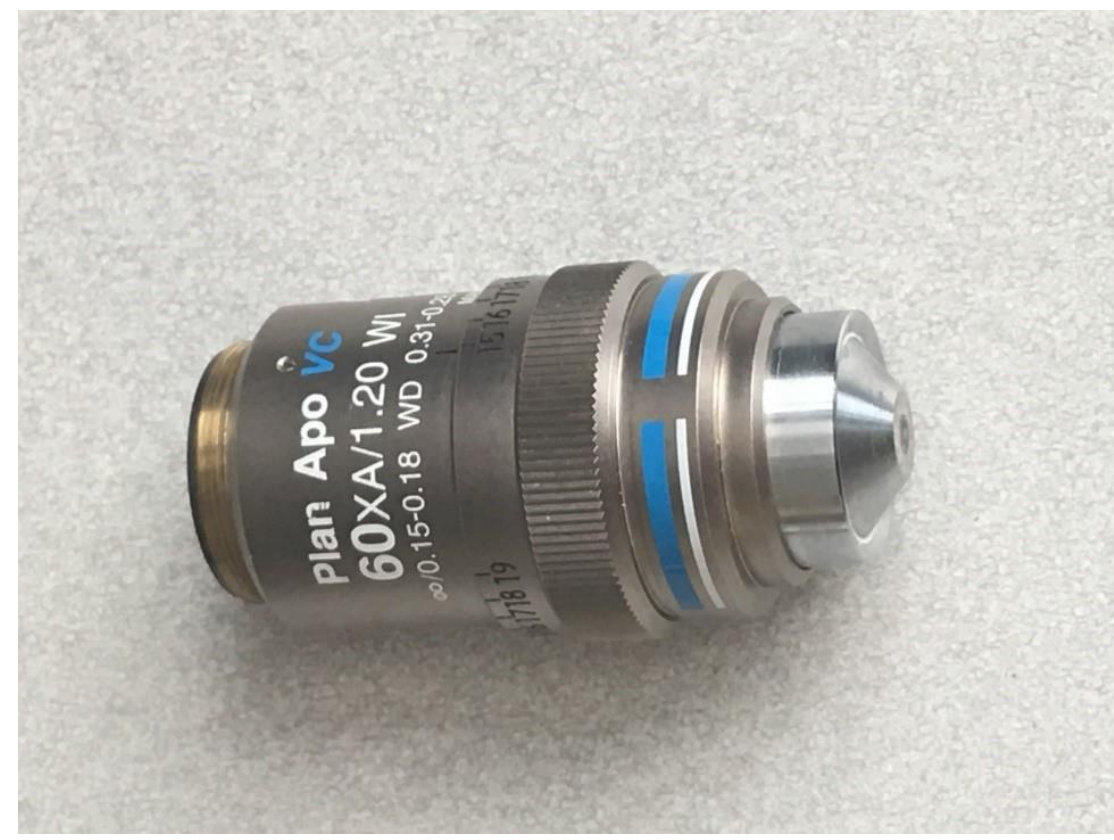

Figure 3. 21 Image of the Nikon $60 \times$ objective lens used in the laser trapping experiments

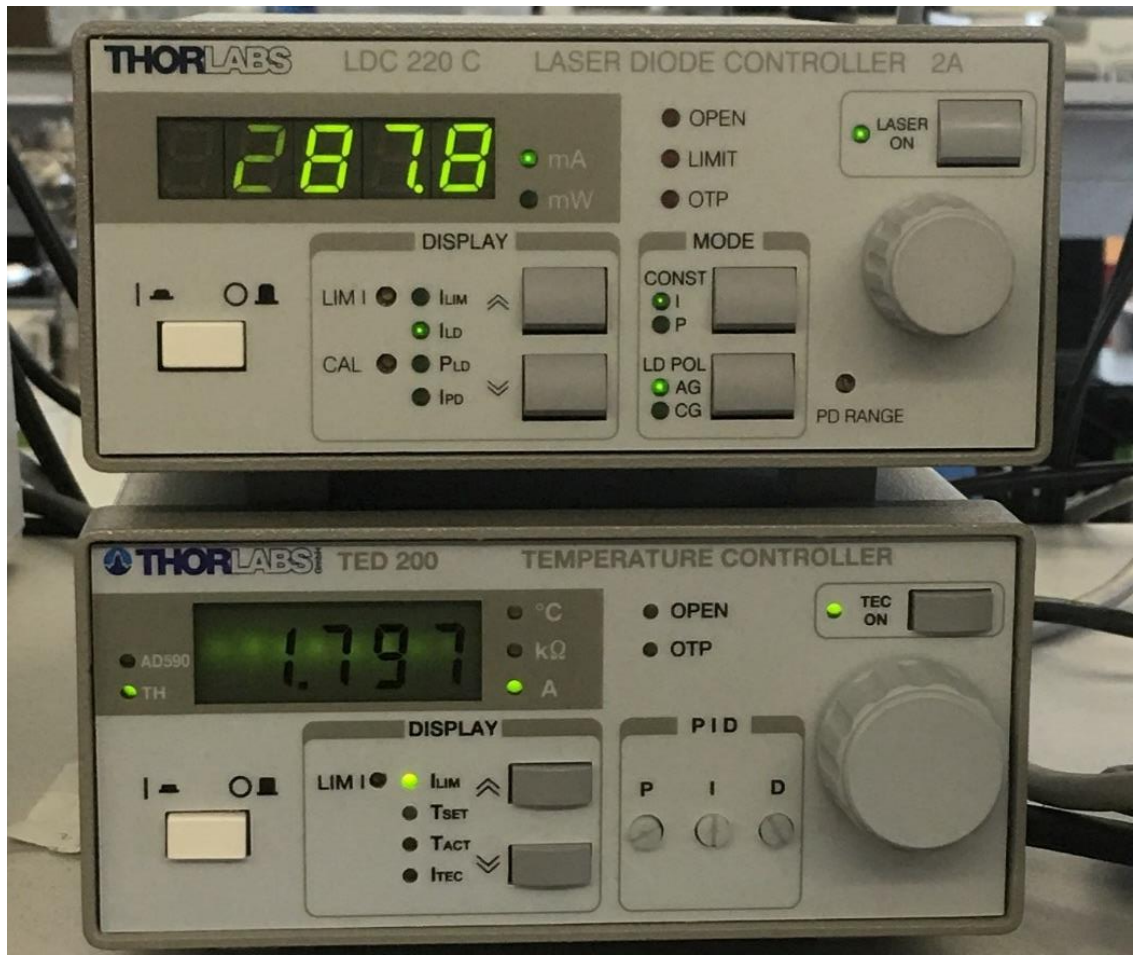

Figure 3. 22 Image of the laser diode and temperature controllers for the laser trapping system 
Initial attempts at cell trapping resulted in a very low yield $(<10 \%)$ for translating cells to the desired locations. The most common reason of failure was the use of culture media with serum, which promoted cell attachment to the glass cover slip. To address this issue, Bovine Serum Albumin (BSA) was added to the micro-chambers prior to loading the cells in the device since BSA prevented cell adhesion to the glass and PDMS substrates. The BSA coating process consisted of the following steps: 1) an appropriate solution of BSA $(10 \mu \mathrm{g} / \mathrm{mL})$ in PBS was prepared; 2) $10 \mu L$ of the coating solution was pipetted into the micro-chamber from the inlet; 3) the platform was swirled around to ensure even coating of the micro-chamber with the BSA solutions; 4) the excess solutions were removed by pipetting; 5) the platform was placed in a petri dish that was then placed into a cell culture incubator for 2 hours; and, 6) the petri dish was transferred to a vacuum chamber and the excess solutions were aspirated before the cells were transferred into the platforms.

After coating the platforms with a layer of BSA, the passaged cells were resuspended in PBS or serum free media and transferred to the platforms using a $1 \mathrm{~mL}$ syringe. In this step, a cell concentration of $10^{7}$ cells $/ \mathrm{mL}$ was used to load the micro-chamber. A relatively low concentration of cells were used to prevent premature apoptosis of the cells or cell death while in the confined micro-chamber space.

In the cell trapping experiment, the laser current was controlled to operate in the range of $0 \sim 300 \mathrm{~mA}$ to prevent cell damage. The general procedure for laser trapping cells included the following steps: 1) the cells were transferred from the flask to the 3-D nano/microfluidic device using a $1 \mathrm{~mL}$ syringe; 2 ) the shutter for the epi-fl illuminator was closed; 3) the filter block rotation control was rotated to make sure that the opening was 
placed into the optical path, 4) the power supply and diascopic illumination lamp were turned on; 5) the platform was placed onto the microscope stage; 6) the laser temperature controller was turned on; 7) the specimen was centered and focused; 8) the laser was turned on and laser current intensity was gradually increased to around $280 \mathrm{~mA}$; 9) the microscope was adjusted to find the focus spot around specimen; 10) the laser was used to select and trap the cells, 11) the camera was turned on and set to the video recording mode; and, 12) the $\mathrm{X}$ and $\mathrm{Y}$ axes of the stage were moved to translate the cells to the desired location.

\subsubsection{Power Supply Instrumentation}

A custom-made instrument was constructed and used to supply a specific pulse width and electric field amplitude to the platform during the electroporation and biomolecule transport experiments. The custom-made power supply instrument included a reed relay and supporting sub-circuits, data acquisition (DAQ) system, power source meter, wave function generator, and software for controlling the pulse amplitude and duration (LabVIEW). The reed relay was used to control one or more reed switches via an electromagnet [130]. The contacts were made of magnetic material, which were sealed in a long and narrow glass tube to avoid corrosion. In this work, a reed relay ( $5 \mathrm{VDC}, 500 \Omega$, 1 A, Aleph America, SD1A05DWJ) was used to make the supporting sub-circuits. There were four pins in this type of reed relay with the two inner pins connected to the wave function generator to provide $5 \mathrm{~ms}, 10 \mathrm{~ms}, 15 \mathrm{~ms}$, and $20 \mathrm{~ms}$ durations of the applied pulse and the outer two pins were connected to the testing platform and a high voltage power supply (maximum $1000 \mathrm{~V}$ ). Once the inner circuit power supply was on, the switch automatically turned on the outer circuit. As a result, the duration of the outer circuit was controlled by the inner circuit AC power supply's frequency. 
The full power supply circuit schematic and printed circuit board layout were designed in Multisim (Figure 3. 23 and Figure 3. 24, respectively.) A switch and a coil together were regarded as the reed relay in the circuit design schematic. The diode in the controlling circuit was used to dampen the coil, while the capacitor in the loading circuit maintained the voltage at a high level. To avoid causing damage to the DAQ USB card, a voltage divider was used to divide the total output voltage into two parts in the loading circuit. The small part, which was $1 \%$ of the total voltage applied to the platform, was less than $10 \mathrm{~V}$ because the maximum input voltage applied to the DAQ was $10 \mathrm{~V}$.

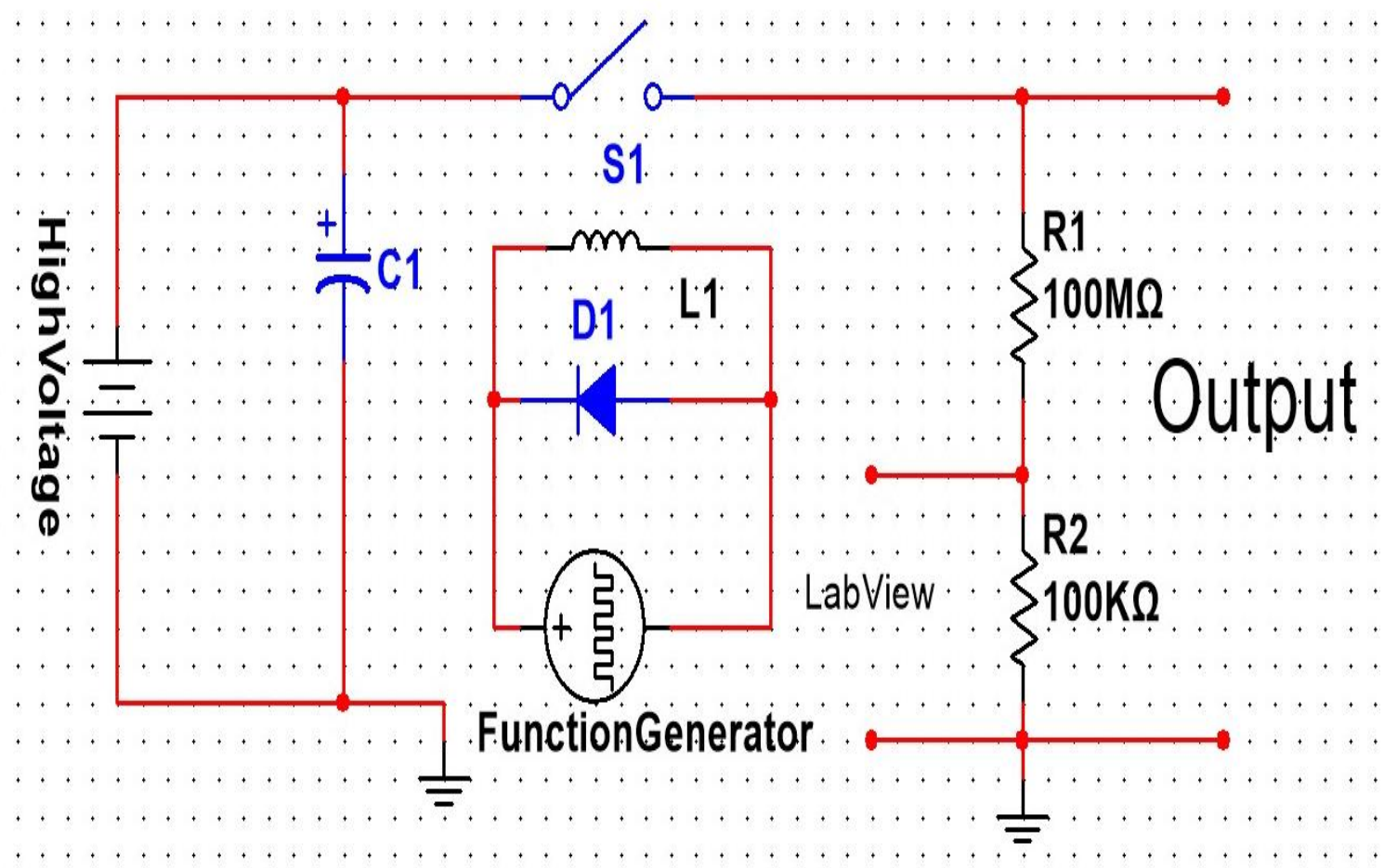

Figure 3. 23 Circuit diagram of the custom-made power supply 


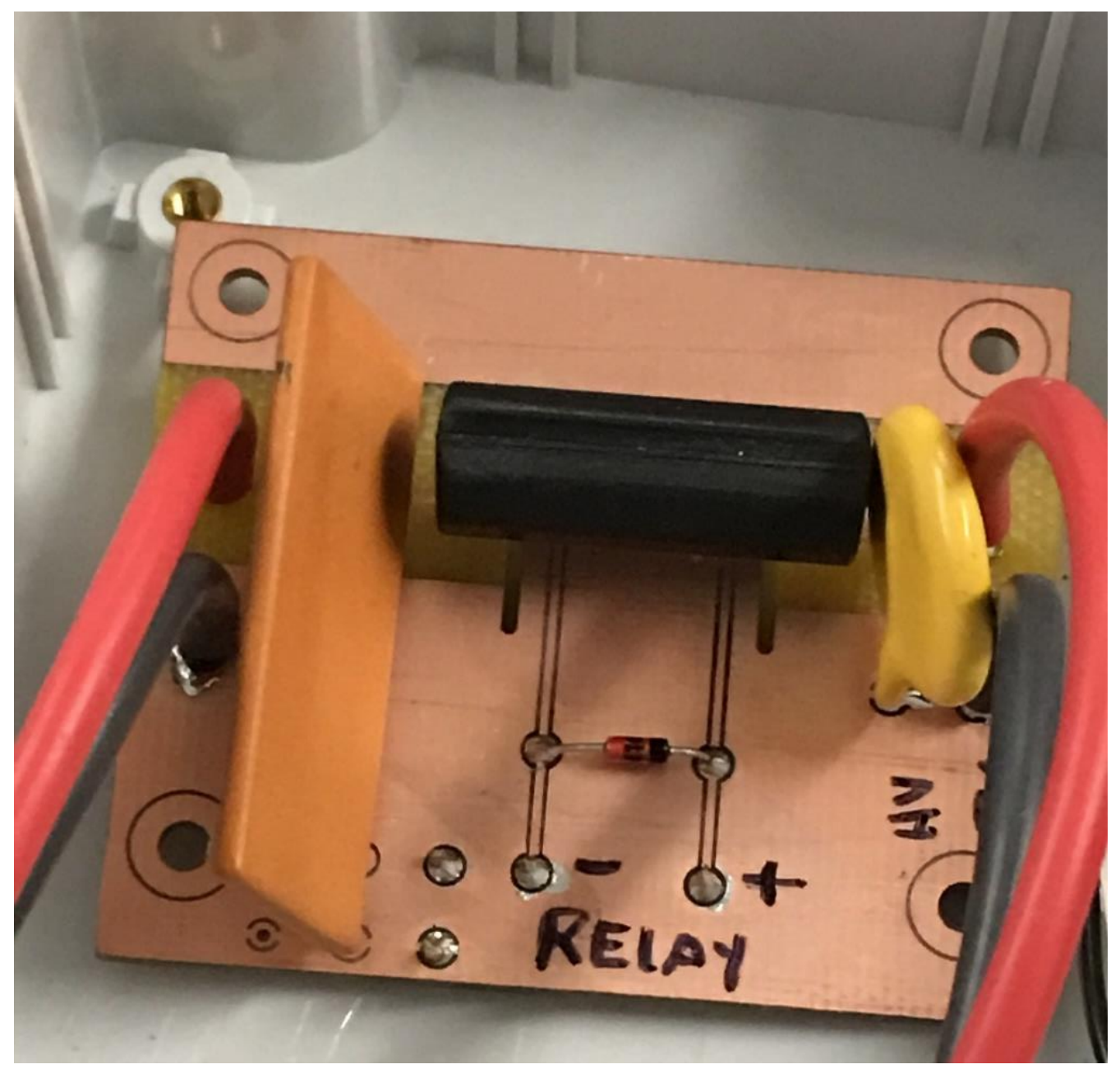

Figure 3. 24 Circuit board layout of the custom-made power supply

Table 3. 5 Electrical connections for the printed board and DAQ channels

\begin{tabular}{|c|c|c|}
\hline Printed Board Pin Number & USB-6009 Channel & Connection \\
\hline 1 & AI0 & Pulse on Device \\
\hline 2 & AI2 & Controlling Pulse \\
\hline 3 & PFI0 & Function Generator \\
\hline
\end{tabular}

The data acquisition card was connected to a custom-made circuit board to provide the physical connections between the inner controlling circuit and the outer loading circuit. 
Two physical channels were used to monitor the real-time pulse applied to the inner controlling circuit and the altered real-time pulse applied to the testing platform (Table 3. 5). An additional physical channel was connected to the sync output of the wave function generator in order to trigger the applied pulse starting at a lower level to acquire a more complete signal in LabVIEW. The full schematic of the physical pin connections for this DAQ USB card was given in Figure 3. 25.

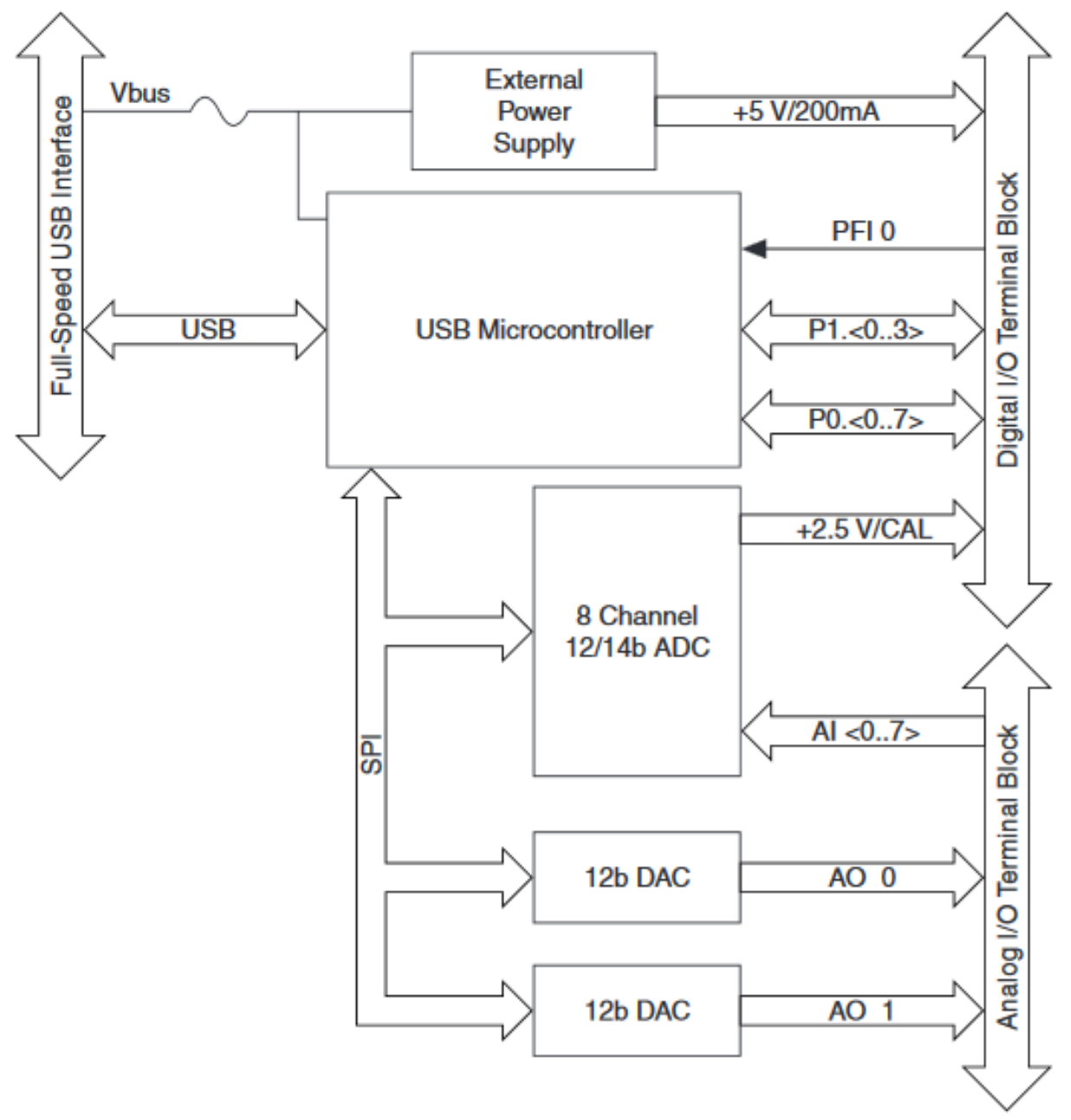

Figure 3. 25 Full schematic of the USB-6009[131] 
The pulse power supply was designed and developed based on a fundamental analysis of the basic electronic components available including the reed relay (ALEPH, SD1A05DWJ, refer to Appendix B), DC power supply source meter (KEITHLEY, 2410 Source Meter, $1100 \mathrm{~V}$ ), waveform function generator (RIGOL, DG1022), and USB-6009 card. As mentioned previously, the main functionality of the custom-made pulse power supply was to provide variable width pulses with controllable amplitudes ranging from 50 $V$ to $1000 \mathrm{~V}$. Fortunately, the LabVIEW software (National Instruments, Austin, TX) was chosen as the development platform to acquire the real-time signal from both the controlling circuit and the loading circuit, since the visual environment in this software not only provided controllability of the data acquisition from a hardware perspective, but also offered user friendly interfaces to easily program the algorithms. Due to the internal resistance of the waveform function generator, USB-6009, and the source meter, the original measured output of the loading circuit (coming from voltage divider) was not proportional to the overall output. An additional, simple algorithm was used in this system to calibrate the final measurement output to ensure that this value was one thousand times less than that of the overall output applied to the platform during electroporation (Figure 3. 26). In addition, a moving average filter program was developed to reduce random noise while retaining a step response since a number of electrical wires were used to connect the instruments and testing platform together (Figure 3. 27). 


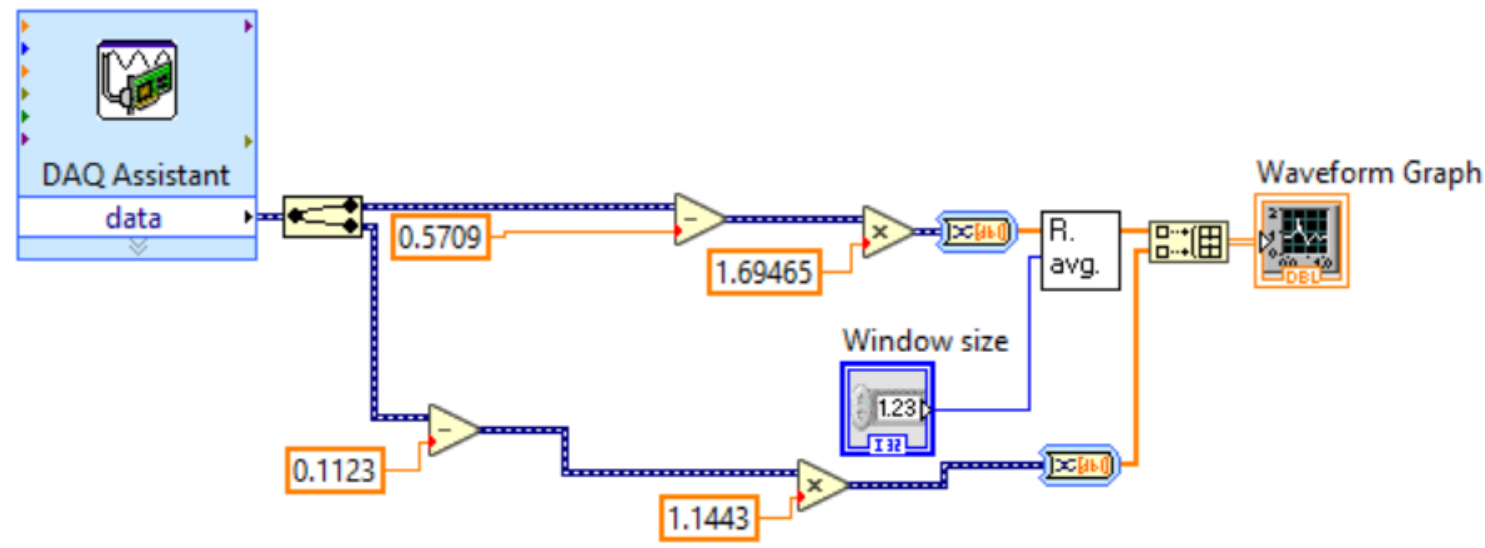

Figure 3.26 Block diagram of the monitoring the real-time pulse algorithm

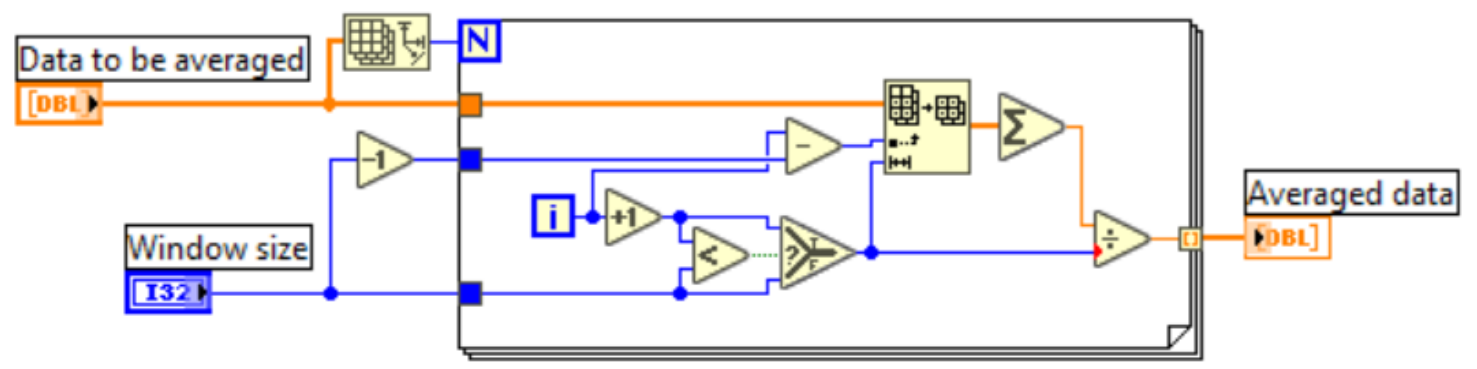

Figure 3. 27 Block diagram of the moving average filter algorithm

\subsubsection{Biomolecules}

In this study, one type of biomolecule was used throughout the experiments, Propidium Iodide (PI) dye (Thermo Fisher Scientific) since the PI dye consisted of negatively charged particles that were easily transported from the cathode side of the micro-chambers through the micro/nanochannel to the anode micro-chamber once a high amplitude pulse was applied. The PI dye was diluted in PBS at $100 \mu \mathrm{g} / \mathrm{mL}$ using $1.0 \mathrm{mg} / \mathrm{mL}$ PI dye and $100 \mu \mathrm{g} / \mathrm{mL}$ PBS. PBS was used instead of DI water to increase the conductivity of the PI dye solution for the electroporation experiments to enhance the creation of a 
nanopore on the cell membrane. As discussed before, the traveling velocity of these biomolecules was the superposition of the PBS or HBSS fluids' electroosmotic velocity and the biomolecules' electrophoretic velocity.

\subsubsection{Confocal Microscope}

Single cell electroporation in the micro/nanofluidic platform was monitored and captured using a Confocal Microscope (Nikon Eclipse Ti) (Figure 3. 28). Compared to

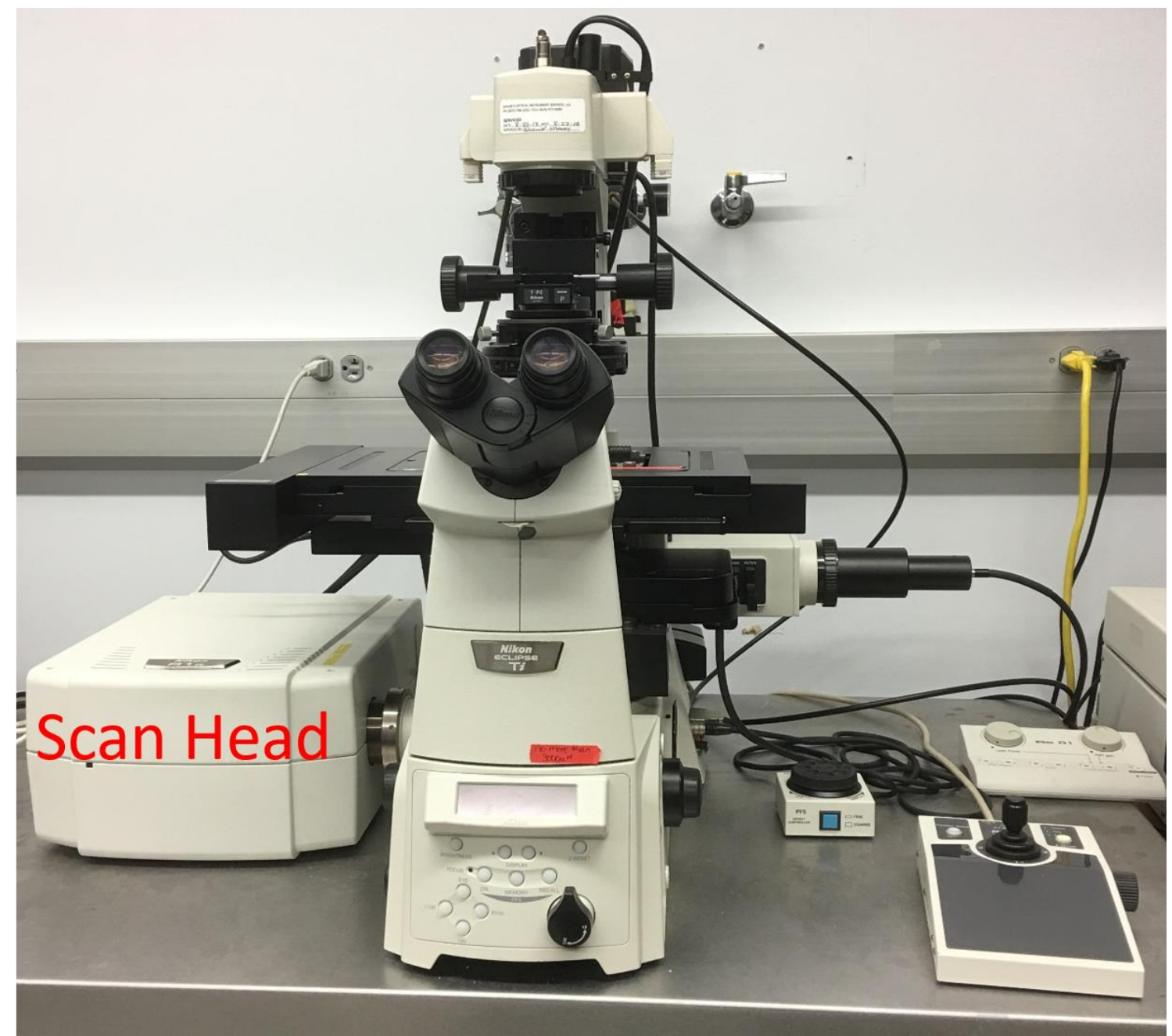

Figure 3. 28 Image of the confocal microscope and scan head (Nikon Eclipse Ti) 


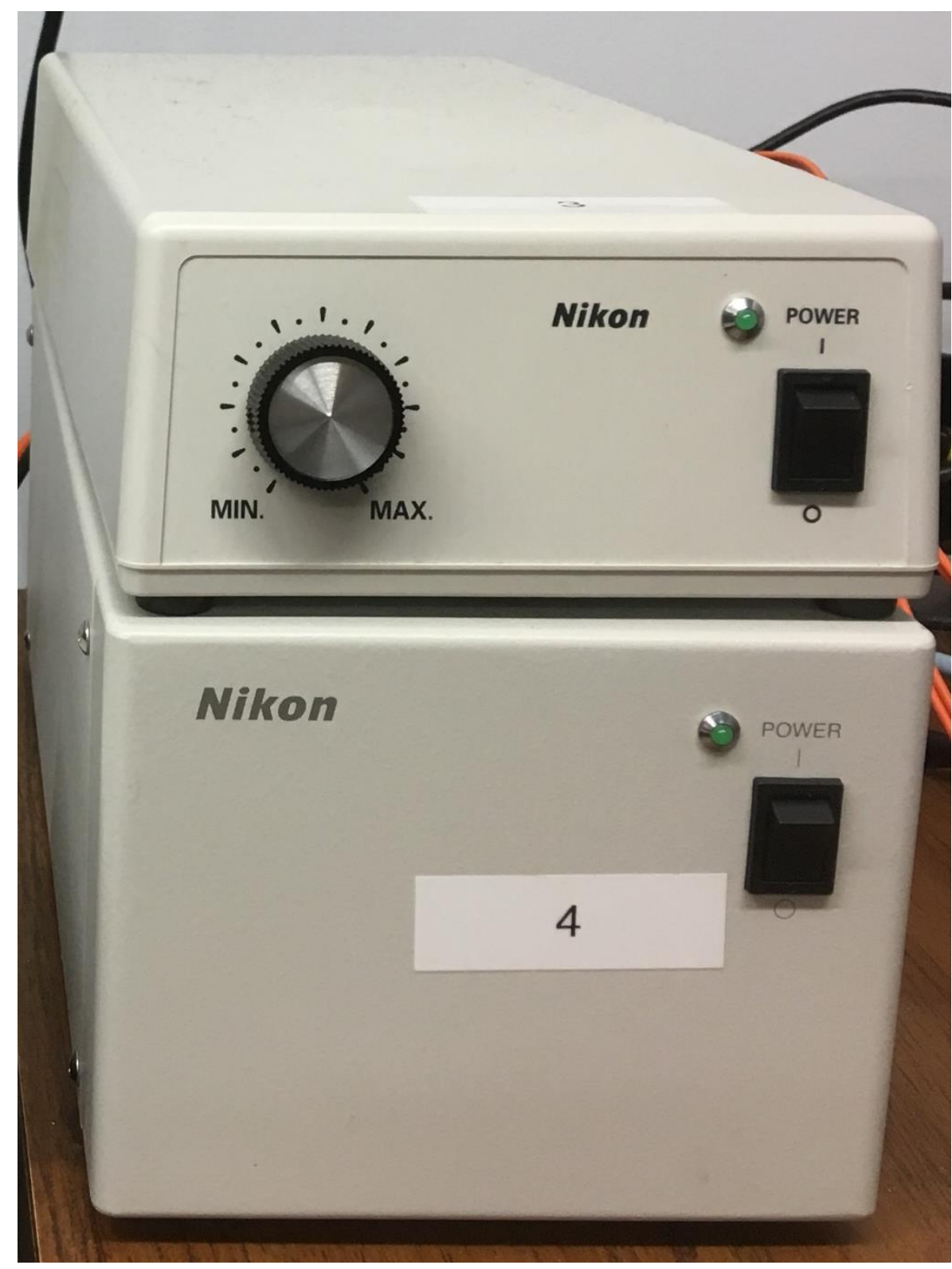

Figure 3. 29 Image of the LED light source for the confocal microscope (Nikon Eclipse Ti)

conventional fluorescent microscopes, the confocal microscope possessed several distinct advantages, including the ability to control the depth of field, reduce or eliminate the background noise from the focus area of the specimen, and improved resolution [132]. The 
key advantage was the pinhole diaphragm, which filtered out the overlapping emission spectra reflected from the specimen. The confocal microscope consisted of an inverted microscope, scan head, confocal detectors, lasers, controllers, LED source, stage controller, Joystick, and PC workstation (Figure 3. 29).

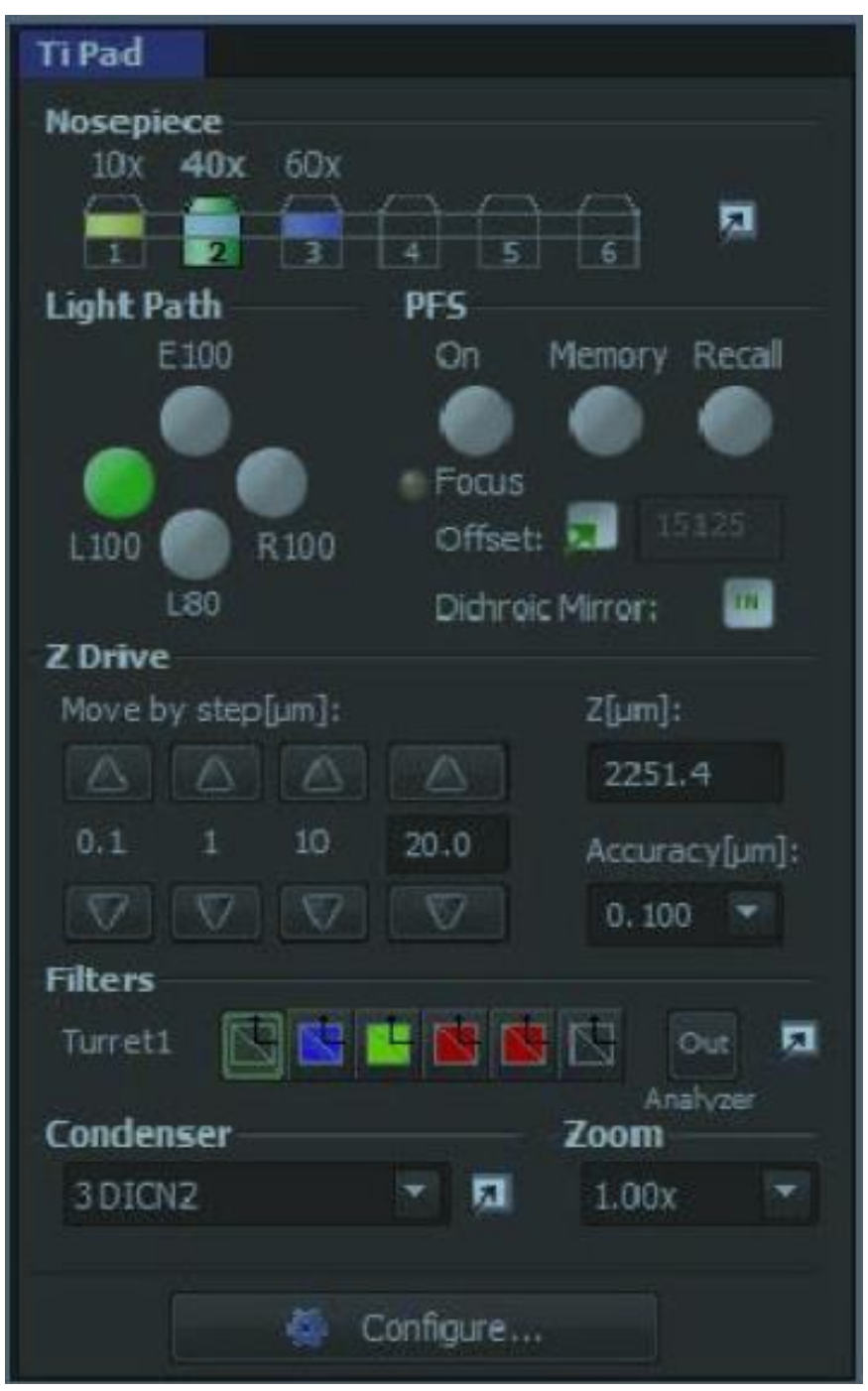

Figure 3. 30 Image of the display panel in the confocal microscope software (Ti Pad) [133] 


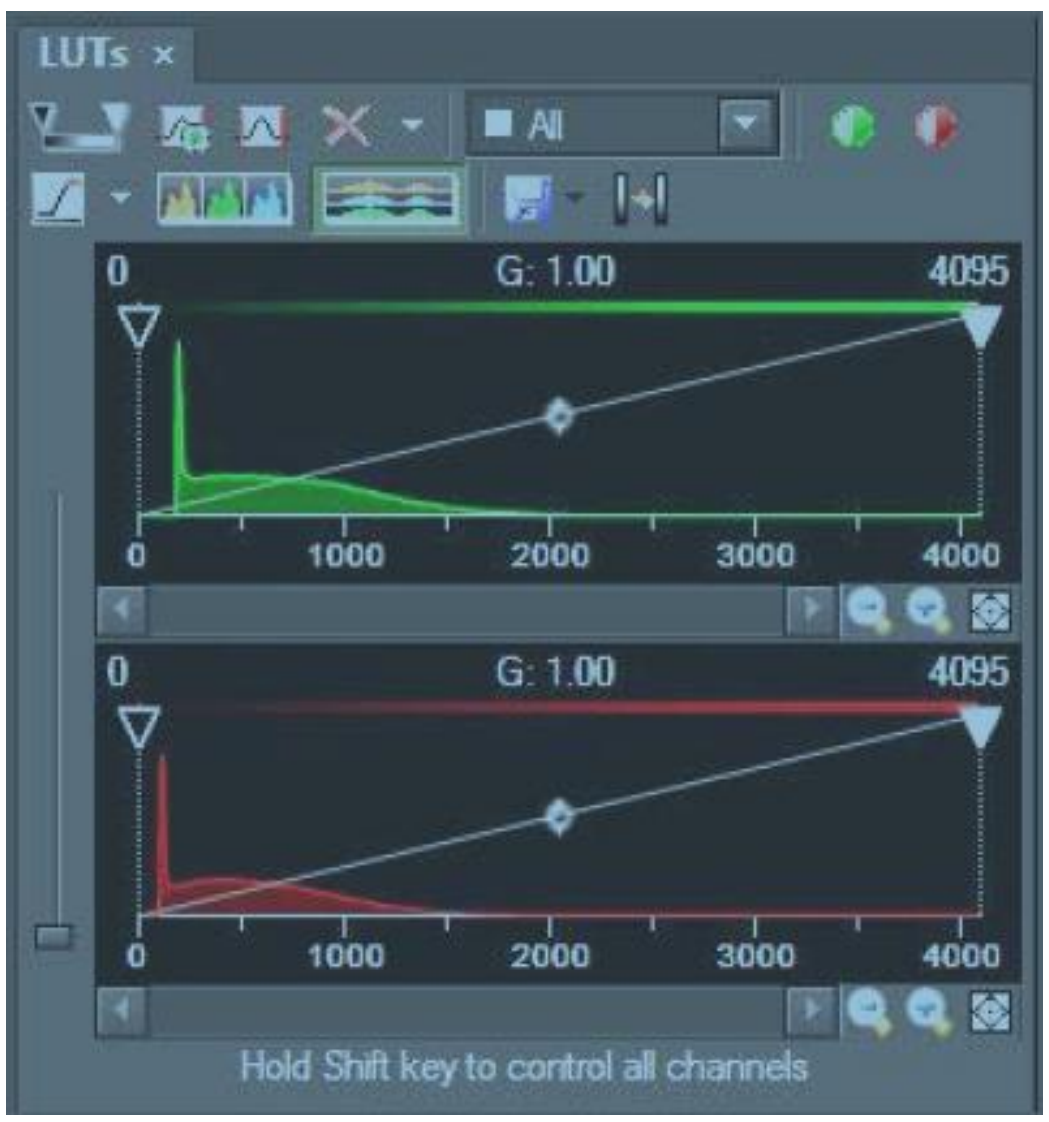

Figure 3. 31 Image of the display panel for the confocal microscope software (LUTs) [133]

The Ti microscope pad (controlling software) displayed all the motorized components on the confocal microscope that could be adjusted in the software (Figure 3. 30) [133]. As shown in Figure 3.30, the focal distance, magnification of the objectives, and condenser were selected using this software. To adjust specimen brightness or contrast, the LUTs menu in the controlling software was utilized (Figure 3. 31). It was also used to indicate and adjust the saturation level of the captured image to acquire a much higher quality image. The electroporation experiments were run based on the amount of time it took to migrate the PI dye into the cell. Using Neutral Density (ND) acquisition (Figure 3. 32) in the controlling software monitored the real-time experimental process by presetting 
the interval time (how long it will take per frame) and the duration (total monitoring time for the whole experiment).

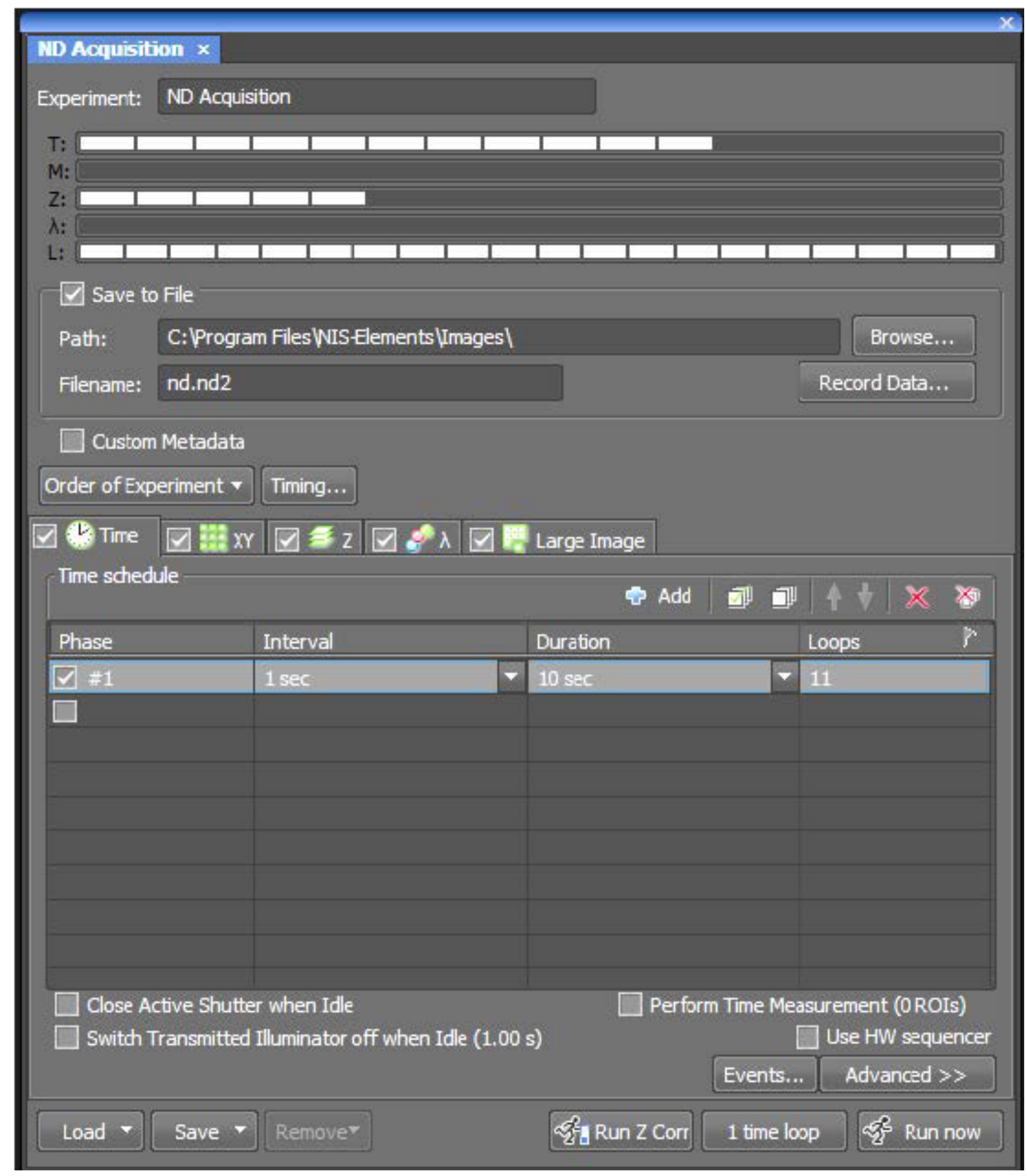

Figure 3.32 Image of the display panel for the confocal microscope software (ND acquisition) [133]

The general procedures for the acquisition of the confocal images or videos included the following steps: 1) the PC workstation, LED light, confocal microscope, lasers, 
controller, and confocal detectors were turned on; 2) the specimen on the glass slide was previewed through the eyepieces and focused on the area of interest; 3 ) four channels + TD mode were chosen; 4) optical configuration was chosen and the interlock was removed; 5) the "Galvano" feature for low FPS (frame per second) and the "Resonant" feature for high FPS were selected; 6) the appropriate laser lines for the specific specimen was selected (we chose TXRED for our electroporation experiments since PI dye were both red); 7) a scan size of $512 \times 512$ at 1 frame per second was chosen; 8) the "Ch series" and "Normal" features were selected for more than one laser lines; 9) the Pinhole feature was set at 1.0 $A U$; and, 10) the "capture" feature was clicked for taking images of the specimen, or the ND acquisition feature was used to record videos of the whole experiment.

\subsubsection{Cell Viability Assay}

Cell viability experiments were performed after the electroporation of the cells with PI dye using the live or dead assay (Calcein Blue AM for live cells, SYTOX green for dead cells). Aliquots of "working" concentrations of the dyes, $1 \mu M$ for SYTOX green (Thermo Fisher, LOT 1816950) and $10 \mu M$ for Calcein Blue AM (Thermo Fisher, LOT: 1832331), were made and frozen for future use. When needed, an aliquot was pulled from frozen storage. A dye solution of $1.1 \mathrm{~mL}$ was made in HBSS with the correct working concentrations. Using a syringe with needle, the dye solution was injected only in the reservoir that holds the cells and into the top wall of the chip right behind the reservoir hole. The dye solution was not injected through the pre-existing reservoir hole, to prevent movement of the cells due to an increased pressure inside the micro-chamber. By injecting the dye into the top wall, this allowed for the dye to flush through the chip and come out 
of the reservoir hole, pushing out any excess PBS. The flushed out solution was vacuumed up and removed. The total dye solution volume of $300 \mu L(0.3 c c)$ was added. The chip was then incubated at $37{ }^{\circ} \mathrm{C}$ for 30 minutes. After incubation, the chip was imaged to identify the cells viability. Images were typically acquired with the $4 x, 10 x$, and $40 x$ objectives depending on the region of the image. When images were taken, an area of interest was identified and put in focus under a bright field filter. Once this image was taken, the blue filter was used to show the green fluorescence in the area of interest and an image was taken. Then, the violet filter was used to show blue fluorescence in the area of interest and an image was taken. The filters were specifically used in this sequential order since the green dye was more readily taken up by the dead cells compared to the blue dye taken up by the live cells. Great care was taken to ensure that the sample was kept in the dark to reduce and prevent photobleaching. 


\section{CHAPTER 4 RESULTS AND DISCUSSION}

In this project, a new system was characterized and optimized to directly-write PMMA fibers on the micron to nanoscale on a variety of substrates. The micro/nanoscale fibers were employed in the manufacturing of a new micro/nanofluidic platform capable of electroporating human cells. A total of 18 micro/nanofluidic electroporation platforms were fabricated and tested. The new micro/nanofluidic platform demonstrated its ability to successfully electroporate live cells as evidenced by confocal microscopy in conjunction with video recording. The research findings of these experiments have been provided below.

4.1 Implementation of a 3-axis Robotic System to Direct-write 3-D Micron/Submicron Fiber Structures

Prior to characterization of the 3-axis robotic system, the fiber drawing capability of the system was compared to previous work by our group using an Ultra-High Precision Micromilling Machine [119, 120] . Compared to previous data [48], the yield for drawing around 60 fibers varying in length from 2.5 to $14 \mathrm{~mm}$ was $100 \%$ for our new process, which was significantly $45.5 \%$ higher than the yield ( $\sim 54.5 \%$ for 66 fibers) obtained with the ultra high-precision micromilling machine at the same polymer solution concentration, fiber lengths, and very similar feed rates. The fiber diameters were also within $\pm \% 22.5$ of the diameters obtained previously. 
As can be seen in the resulting images (Figures 4.1 - 4.3), three-dimensional, freely suspended fibers have been successfully "written" onto the substrates by precisely manipulating the dispenser tip to predetermined spatial locations including the initiating/terminating points as well as points of intersection. The insets of these figures magnified at the intersection points of suspended fibers demonstrated the system's ability to precisely control fiber orientation (Figures $4.1 b-4.3 b)$ in 3-D space. This new system provided considerably more flexibility in creating more complex structures compared to structures created previously by our group (Figure 4.4][119].

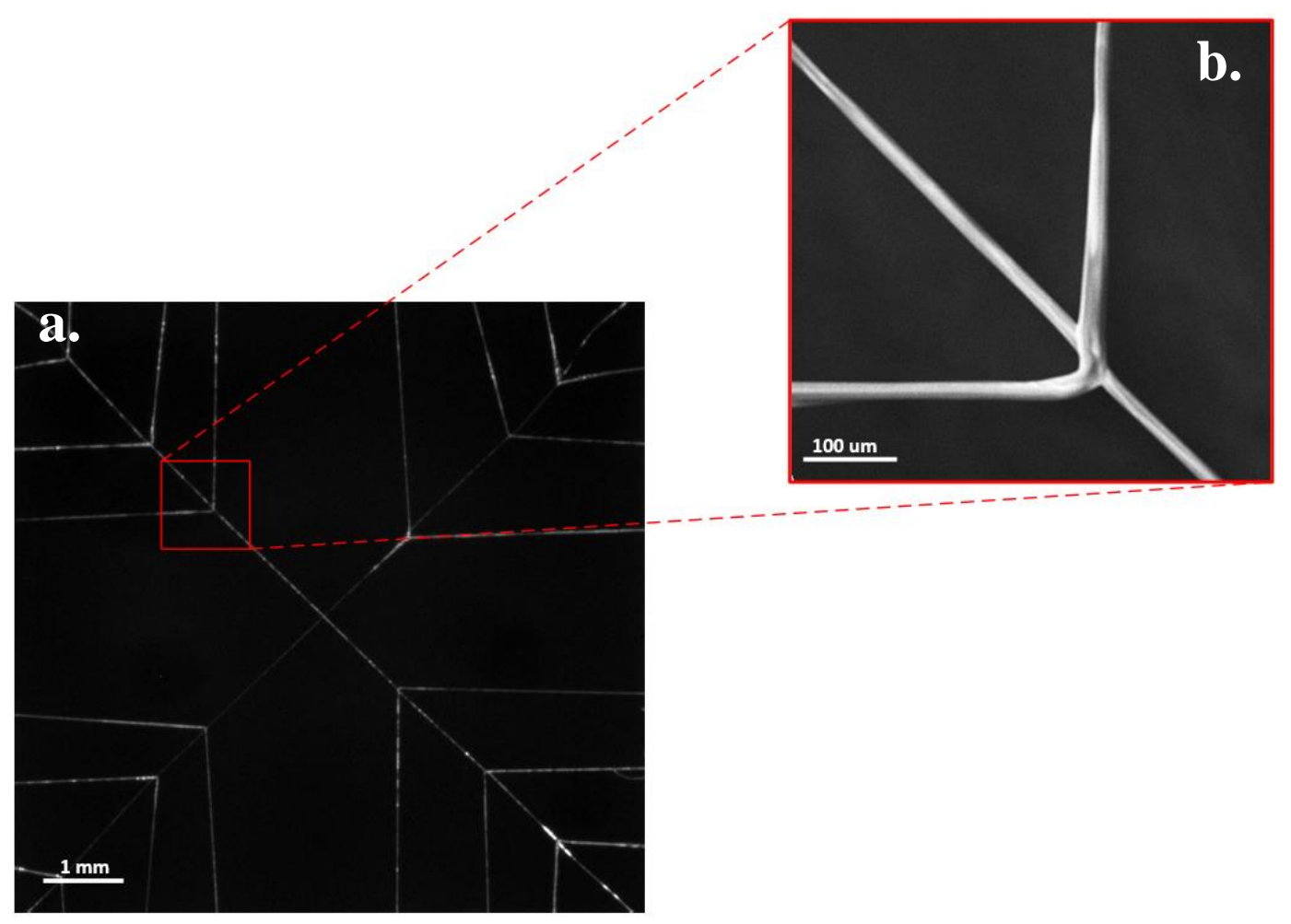

Figure 4. 1 3D suspended structures fabricated from a 24\% PMMA polymer solution: a.) Optical image of "Quad" suspended web structure having 2 support fibers (Diagonals) and 12 bifurcated branched fibers, and b.) SEM image of a single bifurcated fiber (2.0 KV EHT). 


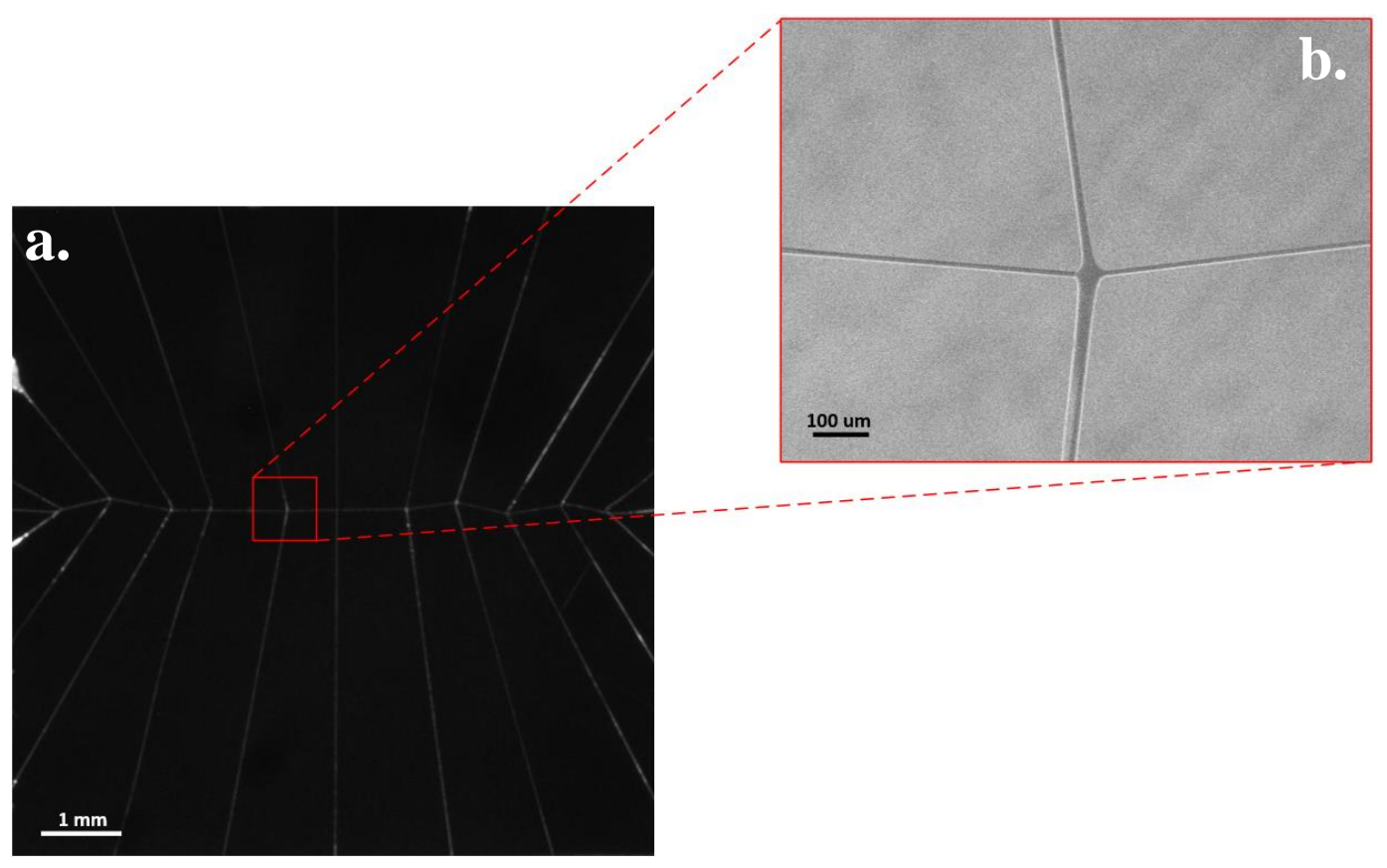

Figure 4. 2 3D suspended structures fabricated from a 24\% PMMA polymer solution: a.) Optical image of "Symmetric" suspended web structure having a single support fiber (Horizontal) and 11 bifurcated branched fibers, and b.) SEM image of a single bifurcated fiber (2.0 KV EHT).

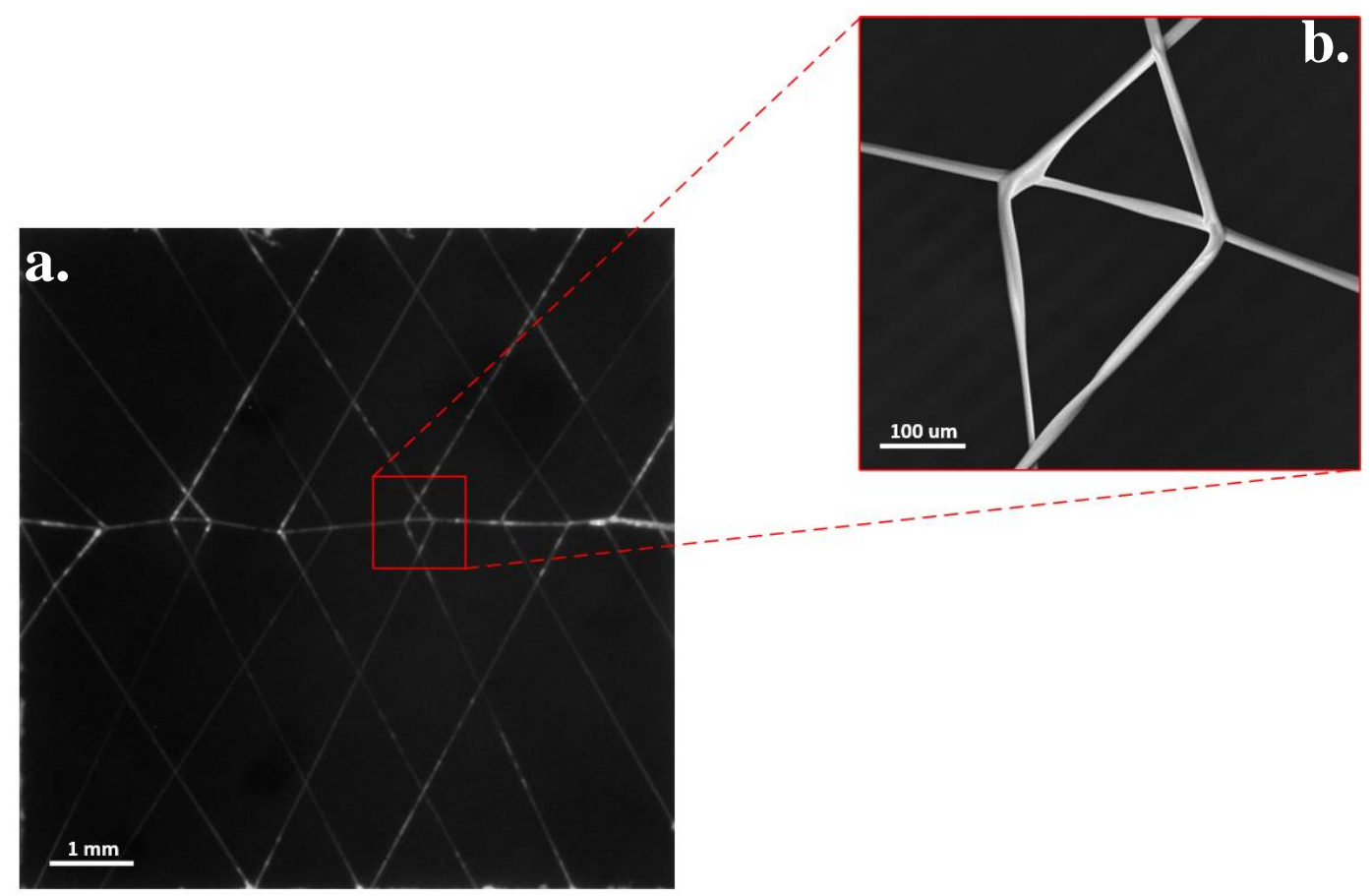

Figure 4. 3 3D suspended structures fabricated from a 24\% PMMA polymer solution: a.) Optical image of "Dual Chevron" suspended web structure having a single support fiber (Horizontal) and 22 bifurcated branched fibers, and b.) SEM image of a single bifurcated fiber. (2.0 KV EHT) 
The sequential point-to-point order of fiber fabrication at which the 3-axis robot and dispensing system generated the freely suspended web-like structures have been shown in Figure 4.5. The arrows represented the robot trajectory during the dispensing process. Micron and submicron polymer fibers drawn on top of one another demonstrated the directwrite system's ability to fabricate wires of varying sizes on a predetermined Figure 4.6.

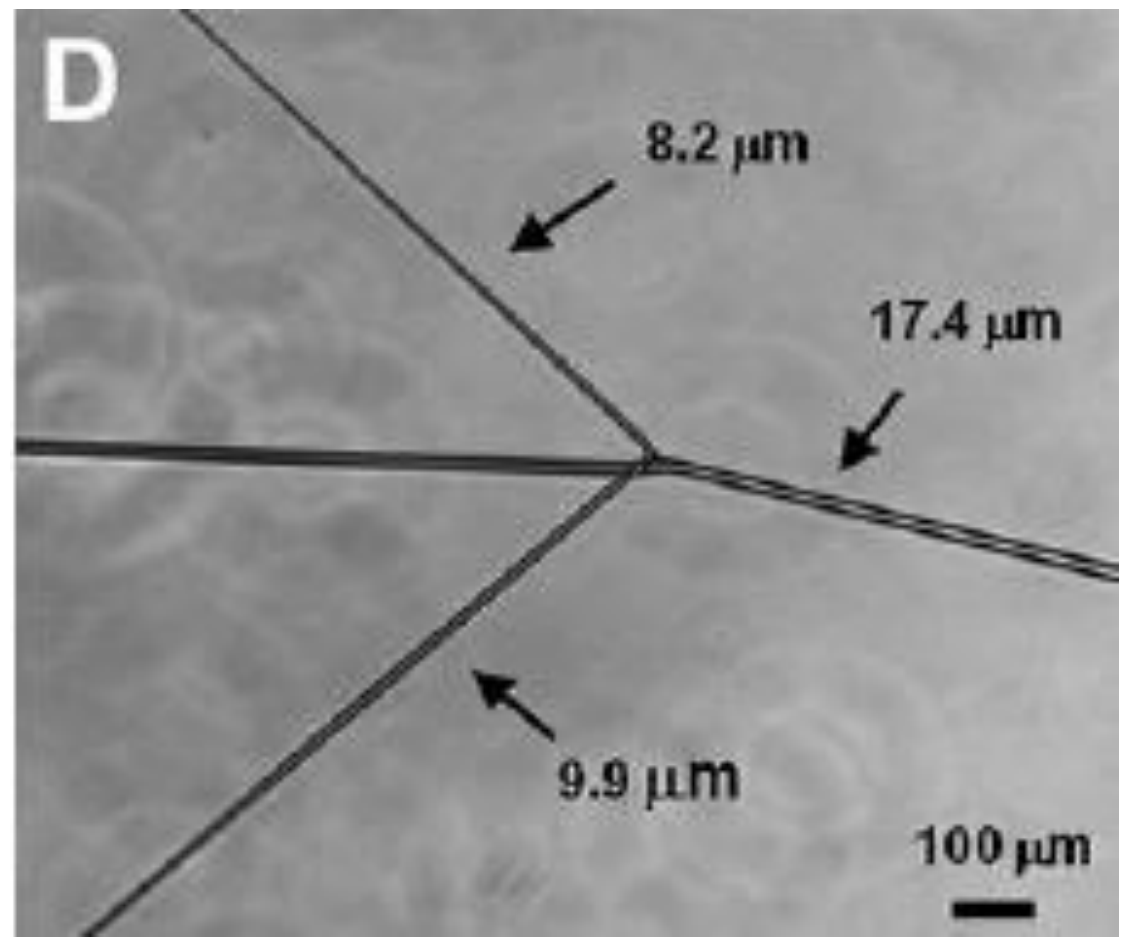

Figure 4. 4 SEM images of suspended, branched fiber structures [119]

Before running each trial, it was critical that the viscosity, mass transfer coefficients and surface tension of the polymer solutions be accurately measured in order to determine whether the robot and dispensing system was capable of processing the polymer to generate the desired fibers. As described previously by our group [48], the polymer solutions must have retained adequate: 1) surface tension to enable the formation of liquid filaments into 


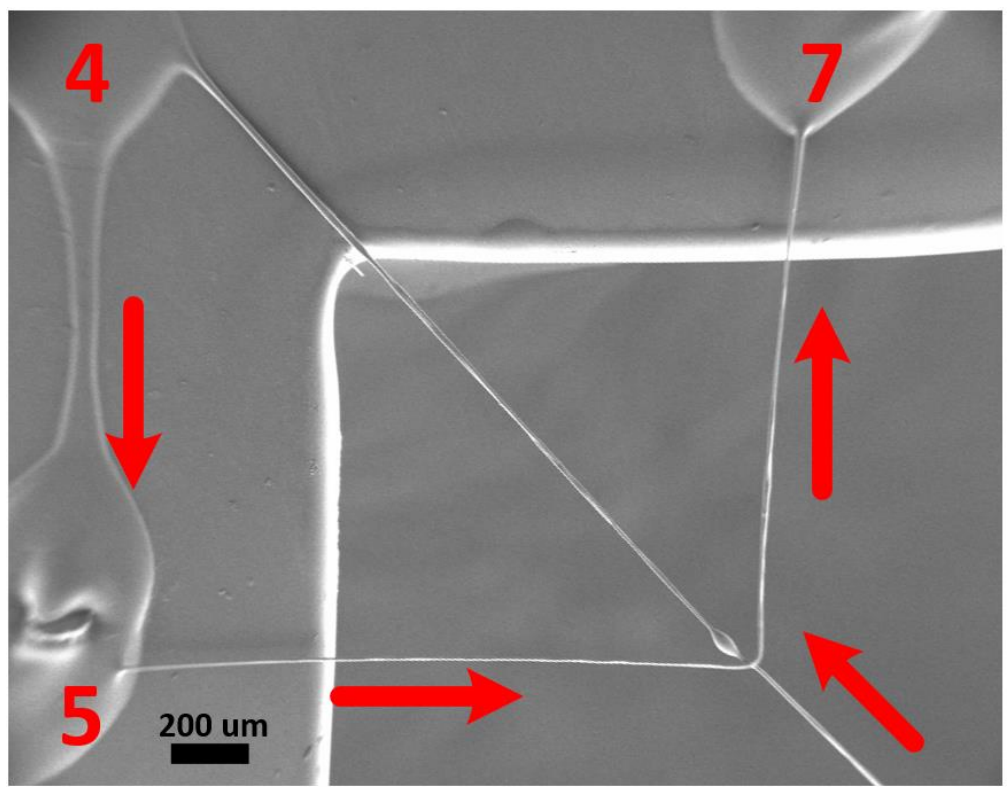

Figure 4. 5 SEM image illustrating the sequential point-to-point order of fabrication of micron and submicron polymer fibers $(2.0 \mathrm{KV}$ EHT).

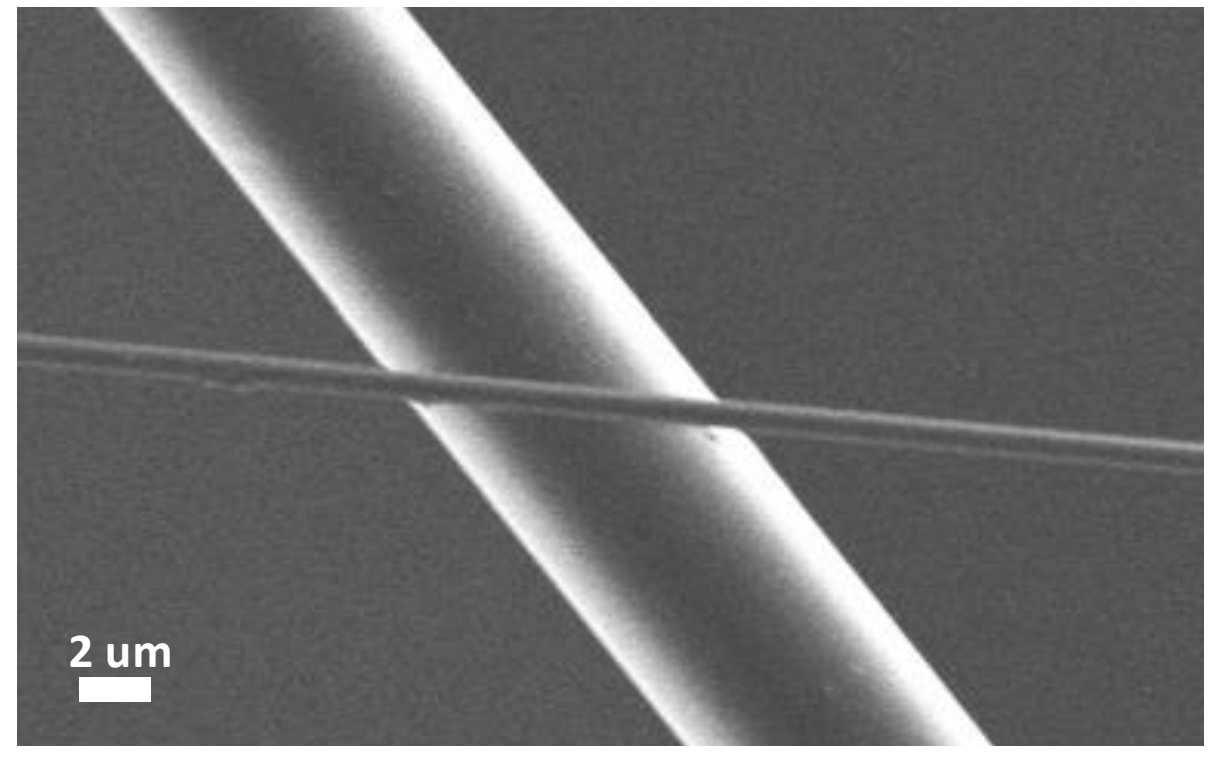

Figure 4. 6 SEM image of micron $(6.5 \mathrm{um})$ and submicron $(555 \mathrm{~nm})$ drawn fibers comprised of $20 \%$ polymer concentration $(2.0 \mathrm{KV}$ EHT $)$. 
micron/sub-micron structures; 2) viscosity to withstand capillary break up; and, 3) evaporation rate to enhance fiber formation. The synergy between these parameters was key to successfully produce fibers over a specified range of diameters. At the same time, instability in any of these parameters prevented the proper formation of the micron/submicron scale fibers. To maintain the synergy between these parameters during fiber fabrication, it was important to ensure that the needle and needle valve were thoroughly cleaned after a direct-write session to prevent: 1) contamination of the solution; 2) reduction in the polymer solution flow rate through the needle; and, 3) excessive growth in the polymer bead at the tip of the needle. In addition, the temperature-controller on the heater was set to the desired temperature to maintain a desired constant evaporation rate of the polymer solution.

For these series of experiments, the bifurcated branch fibers were found to $59 \%$, 41\% and 24\% larger in diameter than that of the support structures in Figures 4.1 - 4.3, respectively, using the $24 \%$ PMMA solution. This was primarily due to the distance at which the fibers were drawn. Specifically, the support structures were drawn across the entire width of the substrate (10.0 $\mathrm{mm}$ in $\mathrm{X}$ and $\mathrm{Y}$ directions; $14.4 \mathrm{~mm}$ diagonally). As a result, the support structures were the longest fibers of the overall suspended structures and the bifurcated branch structures were shorter, ranging from $7 \mathrm{~mm}$ maximum length down to $2.5 \mathrm{~mm}$. This shorter fiber drawing length did not effectively induce the fiber strain required during the fiber thinning process to produce the small diameter fibers. On the other hand, larger diameter fibers were required to serve as the support structures in order to effectively sustain the tugging and deformation induced during the bifurcated branch drawing process. As the bifurcation branches were drawn across the support fibers, a 
remodeling of the support fiber geometry occurred due to the drawing forces as well as a localized dissolution of the PMMA polymer at the interface between the support and branched fibers from the solvent present in the polymer solution. Thus, in some instances, the support fibers needed to be fabricated from a polymer solution consisting of a higher concentration of polymer to produce larger diameter and mechanically stronger fibers.There were primarily three effective methods for modifying the existing protocol to generate a wider range of support and branched fiber diameters: 1) initially dispense the polymer from a larger needle tip $($ e.g., 25 gauge; ID $=254 \mu m)$ to generate the support fibers and then exchange for a smaller needle tip (e.g., 32 gauge; ID = $101.6 \mu m)$ to fabricate the smaller branched fibers; 2) as noted above, use multiple polymer concentrations; and/or, 3) adjust the feed rate, i.e., the speed at which the stage traverses, where the increasing feed rate produces smaller diameter fibers and decreasing feed rates create larger diameter fibers.

One limitation of the automated direct-write process was that only one concentration of polymer solution was dispensed at a time. This restricted the level of complexity of the suspended structures to be developed without having to: 1) add a second dispensing valve to the robot; or, 2) remove the existing valve and perform the cleaning protocol before dispensing the second polymer solution, which takes additional time. A second limitation was the feed rate (or print speed), where the maximum feed rate that the system was capable of achieving is $500 \mathrm{~mm} / \mathrm{s}$. However, there was a tradeoff between feed rate and fiber formation. Specifically, if the inertial forces (forces due to the feed rate) were greater than the surface tension forces and evaporation rate of the polymer solution, fiber formation did not occur. On the other hand, if the feed rate was too low, fibers would 
fracture due to excessive evaporation before and during the elongation process. Thirdly, the fiber and structure dimensions were limited to the operating range of the stage of the robot in the $\mathrm{x}, \mathrm{y}$ and $\mathrm{z}$ directions at $200 \mathrm{~mm}, 200 \mathrm{~mm}$ and $25 \mathrm{~mm}$, respectively, with a $5 \mu \mathrm{m}$ positional accuracy. Nonetheless, this process did enable the formation of high aspect ratio (fiber length: diameter) fibers. Strategically varying the dispensing tip-size and the polymer solution concentration allowed for a broader fiber diameter range, creating the ability to generate freely suspended structures with a high level of complexity.

\subsection{Characterization of Direct-write Sub-micron Fibers}

A novel direct-write method via an advanced 3-axis robotic dispensing system has been introduced that produced repeatable and controllable sub-micron/nano scale fibers by controlling several key process variables. Some factors, including the initiation and termination positions, feed rates, and length of fibers were precisely manipulated using the 3-D robot software, while other factors such as dispense time, needle size, and dispensing chamber pressure - which pushes the fiber solution out from the needle tip - were adjusted by the dispensing system.

\subsubsection{PMMA Solution's Physical Properties}

In order to understand the underlying mechanism behind the thinning dynamics of polymer fluids, the effect of surface tension, viscosity, dispense time, air pressure, and mass transfer coefficient of the polymer solutions were investigated via characterizing the fiber drawing process by varying each of these parameters and analyzing the significance of each of these variables using ANOVA algorithms in Minitab and regression equation 
analysis in Matlab. The empirical prediction equation was generated from ample experimental data illustrating the general trend of fiber diameter at the specific controlling parameters. As described in the Methods section, PMMA solution viscosities at different weight concentrations were measured using LVDV-II+ and RVDV-II+ viscometers interfaced with a cone-and-plate. Low viscosity fluids were measured with the LVDV-II+ viscometer since it has limited measurement range, while high viscosity fluids were measured with the RVDV-II+ viscometer. Mass transfer coefficients of the PMMA solutions were measured using thermogravimetric analysis, to record high-precision weight measurements at different temperatures. The surface tension of PMMA solutions were measured by dipping a glass rod into test sample PMMA solutions as described by the

Table 4. 1 Physical properties of PMMA solutions at different weight concentration.

\begin{tabular}{clll} 
Concentration & $\begin{array}{l}\text { Viscosity } \eta \\
\left(P a^{*} s\right)\end{array}$ & $\begin{array}{l}\text { Mass } \\
\text { transfer } \\
\text { coefficients } \chi(\mathrm{m} / \mathrm{s})\end{array}$ & $\begin{array}{l}\text { Surface } \\
\text { tension } \sigma(\mathrm{mN} / \mathrm{m})\end{array}$ \\
\hline $19 \%$ & $2.94 \pm 0.22$ & $(9.07 \pm 0.62) \times 10^{-8}$ & $87.50 \pm 10.70$ \\
$20 \%$ & $7.49 \pm 0.46$ & $(9.07 \pm 0.62) \times 10^{-8}$ & $106.69 \pm 23.91$ \\
$21 \%$ & $8.29 \pm 0.10$ & $(9.07 \pm 0.62) \times 10^{-8}$ & $106.85 \pm 12.20$ \\
$22 \%$ & $13.39 \pm 0.47$ & $(9.07 \pm 0.62) \times 10^{-8}$ & $105.39 \pm 24.40$ \\
$23 \%$ & $32.16 \pm 0.70$ & $(9.07 \pm 0.62) \times 10^{-8}$ & $152.23 \pm 53.82$ \\
$24 \%$ & $37.34 \pm 4.65$ & $(9.07 \pm 0.62) \times 10^{-8}$ & $262.01 \pm 42.44$ \\
$25 \%$ & $45.22 \pm 1.17$ & $(9.07 \pm 0.62) \times 10^{-8}$ & $234.20 \pm 12.69$ \\
$26 \%$ & $64.41 \pm 1.08$ & $(9.07 \pm 0.62) \times 10^{-8}$ & $366.2 \pm 10.25$ \\
$27 \%$ & $94.16 \pm 1.55$ & $(9.07 \pm 0.62) \times 10^{-8}$ & $500.60 \pm 69.28$ \\
\hline
\end{tabular}


Wilhelmy technique. All three physical property parameters for the PMMA polymer solutions at different weight concentrations ranging from 19\% 27\% have been shown in Table 4.1. Generally, viscosity and surface tension increase as the PMMA polymer solution's concentration increases. For $22 \%$ concentration, the surface tension is a little lower than $21 \%$ concentration's since PMMA powder at this concentration was not completely dissolved in solvent when they were testing. Mass transfer coefficients for each concentration stay at the same value due to using the same solvent for all the testing solutions.

\subsubsection{Statistical Analysis Results}

Four groups of comparison experiments were executed to characterize the directwrite process by changing four factors: 1) needle size; 2) dispense time; 3) concentration of PMMA; and, 4) feed rates. In group one experiments, weight concentration, dispense time, and feed rates were maintained at a specific value, and needle size and fiber length were varied in order to determine how needle size and fiber length affected the final performance of the written PMMA fibers. In a similar way, dispense time, concentration, and feed rates, as well as fiber length, were changed to figure out how each one of them influenced the final fiber diameter.

The data presented in the above four graphs showed that fiber diameters generally became smaller as the fiber lengths increased under any experimental conditions (Figures 4.7 - 4.10). With a 32 gauge needle, the fiber diameters were all smaller for different lengths than that of the other three different gauge needles from Figure 4.7 because few amounts of polymer solutions were discharged from smaller gauge needle. For the case of the dispense time (Figure 4.8), a shorter dispensing time usually led to smaller diameters 


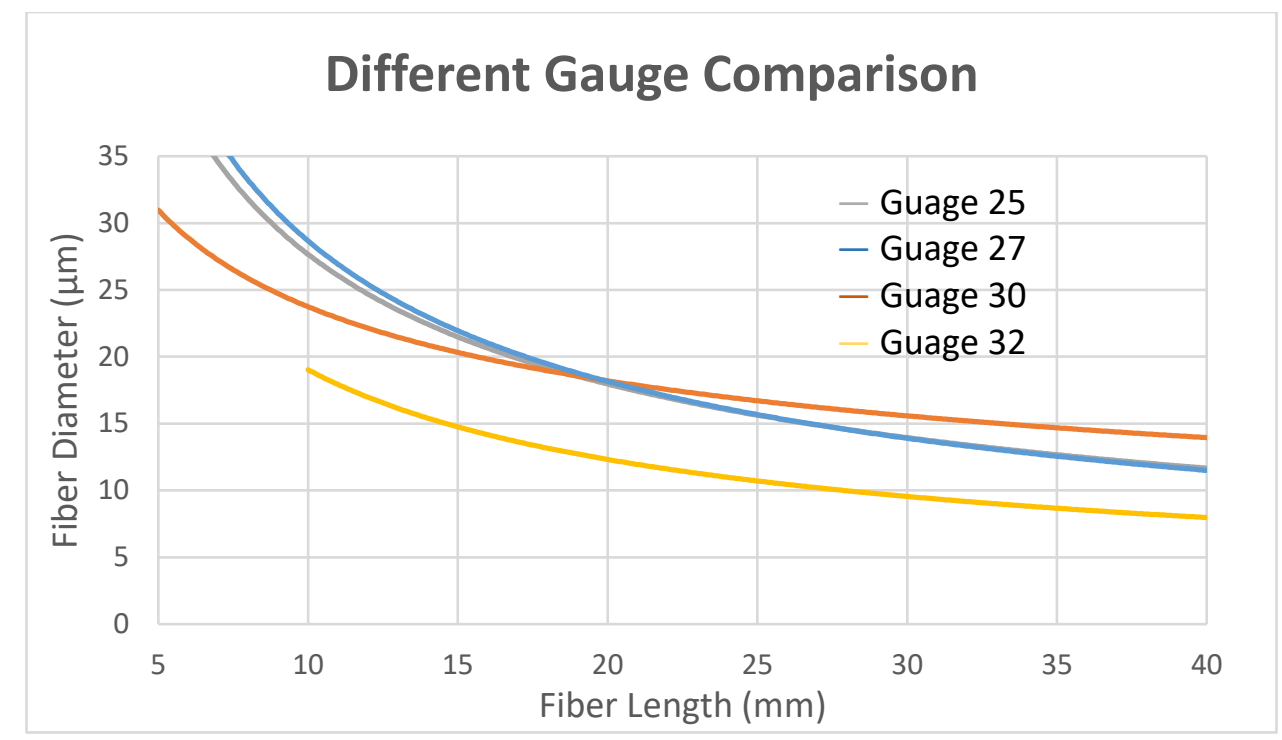

Figure 4. 7 Graph demonstrating the effect of the fiber length on the drawn fiber diameter for varying needle gauge sizes of $25,27,30,32$ using $23 \%$ weight concentration, 0.02 s dispensing time and $500 \mathrm{~mm} / \mathrm{s}$ feed rates.

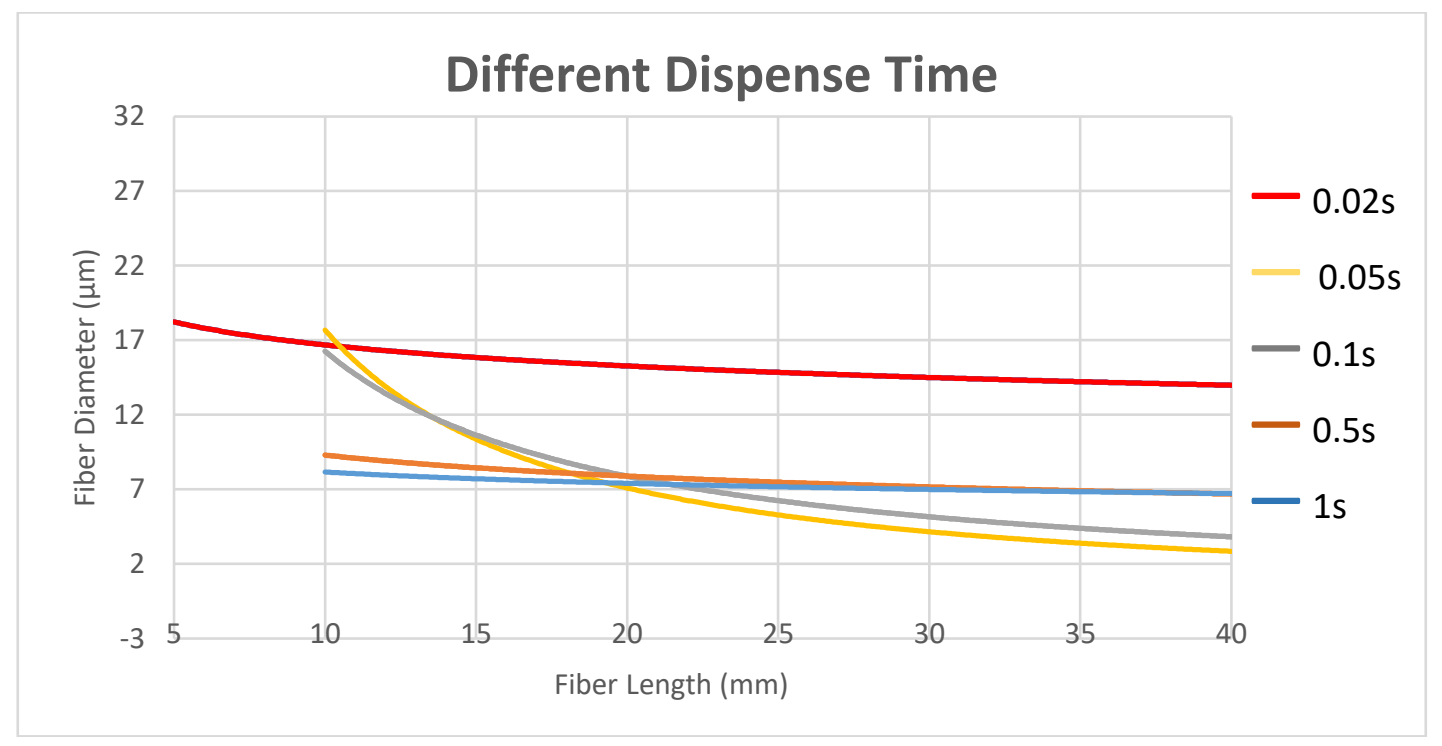

Figure 4. 8 Graph demonstrating the effect of fiber length on drawn fiber diameter for dispense times of $0.02,0.05,0.1,0.5,1$ using $23 \%$ weight concentration, 32 gauge needle and $500 \mathrm{~mm} / \mathrm{s}$ feed rates. 


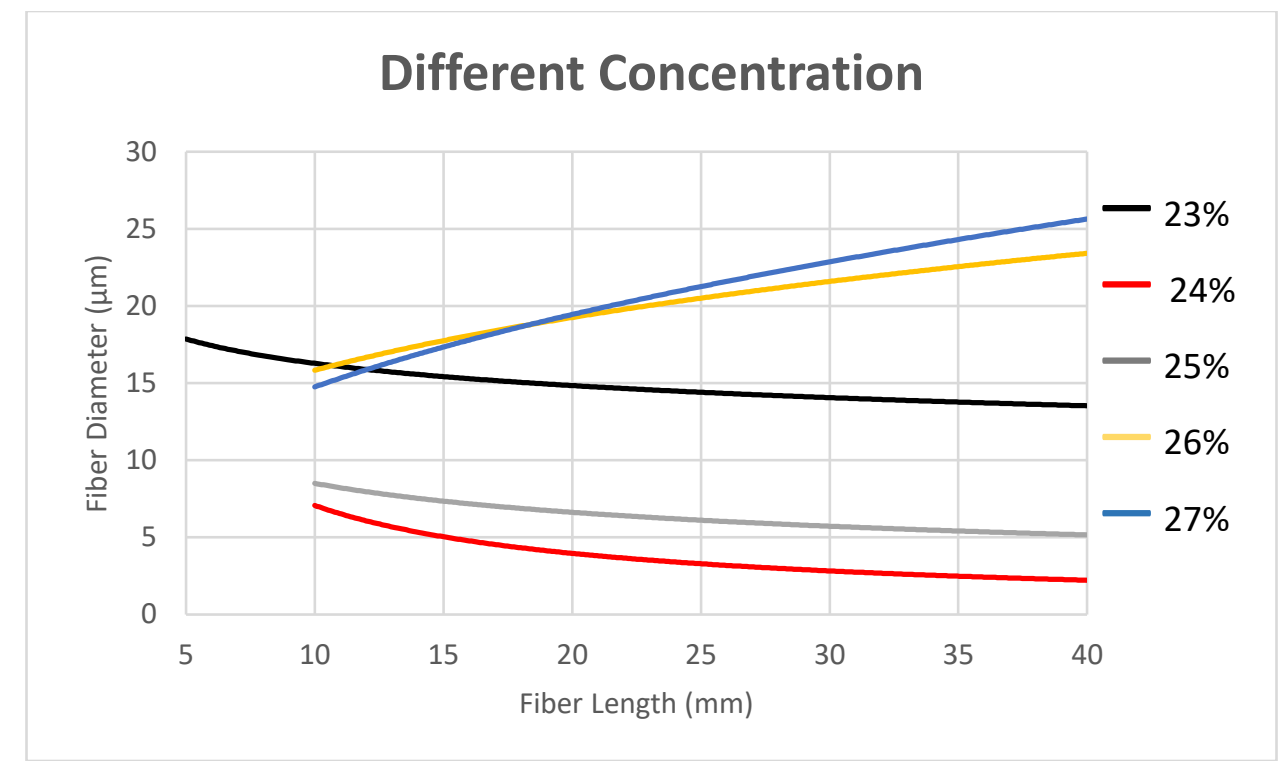

Figure 4. 9 Graph demonstrating the effect of fiber length on drawn fiber diameter for concentrations of $23 \%, 24 \%, 25 \%, 26 \%, 27 \%$ using $0.02 \mathrm{~s}$ dispensing time, 32 gauge needle and $500 \mathrm{~mm} / \mathrm{s}$ feed rates.

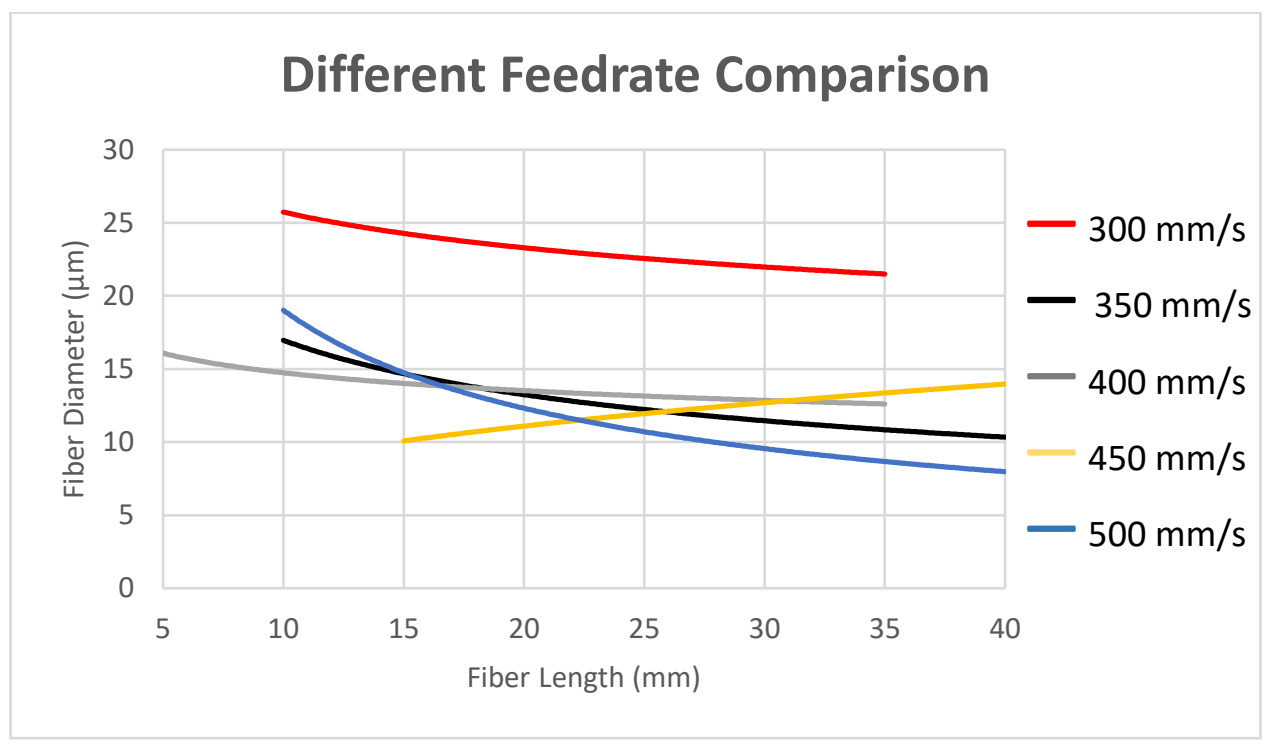

Figure 4. 10 Graph demonstrating the effect of fiber length on drawn fiber diameter for feed rates of 300, 350, 400, 40, 500 using $0.02 \mathrm{~s}$ dispensing time, 32 gauge needle and $23 \%$ weight concentration.

since a short dispensing time resulted in less PMMA solution being expelled from the needle; thereby, a smaller diameter bead extruded out of the needle tip. Figure 4.9 
illustrated that a lower concentration of PMMA solutions produced smaller diameter fibers compared to high concentrations of PMMA solutions such as $26 \%$ and $27 \%$ because the ratio of surface tension changing rates to viscosity changing rates increased as the concentration became higher from Table 4.1(lower ratio results in larger diameter in Equation 2.6). A group of experiments covering a range of feed rates, Figure 4.10 indicated that smaller diameter fibers corresponded with higher feed rates since bigger force was applied for higher speed (feed rates) on the polymer fiber during elongation.

Analysis of variance (ANOVA) was used to determine the most significant factors from the five different factors mentioned above to further optimize the experimental process. The dispense time, fiber length, concentration and needle size were found to be the most important factors via ANOVA analysis, while feed rate was not determined to be a key factor in the determination of the fiber diameter. Based on these analysis results, additional experiments were performed to further elucidate the effect of feed rate and fiber length on the process in order to optimize the experiment in the next characterization process. Although high feed rates corresponded to smaller diameter fibers, the feed rates still needed to be decreased in future experiments for two reasons: 1) high feed rates resulted in low yield since the filaments/fibers would break in most cases during writing process; and, 2) low feed rates enabled longer evaporation time, which provided the filament with enough time to form and thin during the writing process. On the other hand, the fiber drawing length was varied from $22 \mathrm{~mm}$ to $40 \mathrm{~mm}$ in $2 \mathrm{~mm}$ increments with two fibers being drawn for each length (Figure 4.10). With these new experiments, an ANOVA was again performed and the length $(\mathrm{p}=0.000)$, needle size $(\mathrm{p}=0.000)$, concentration $(\mathrm{p}=0.000)$ and dispense time $(\mathrm{p}=0.001)$ were found to be significant factors. Subsequently, 
a prediction equation (Equation 3-1) was also yielded from the ANOVA analysis, which demonstrated the relative impact of each parameter on the fiber diameter.

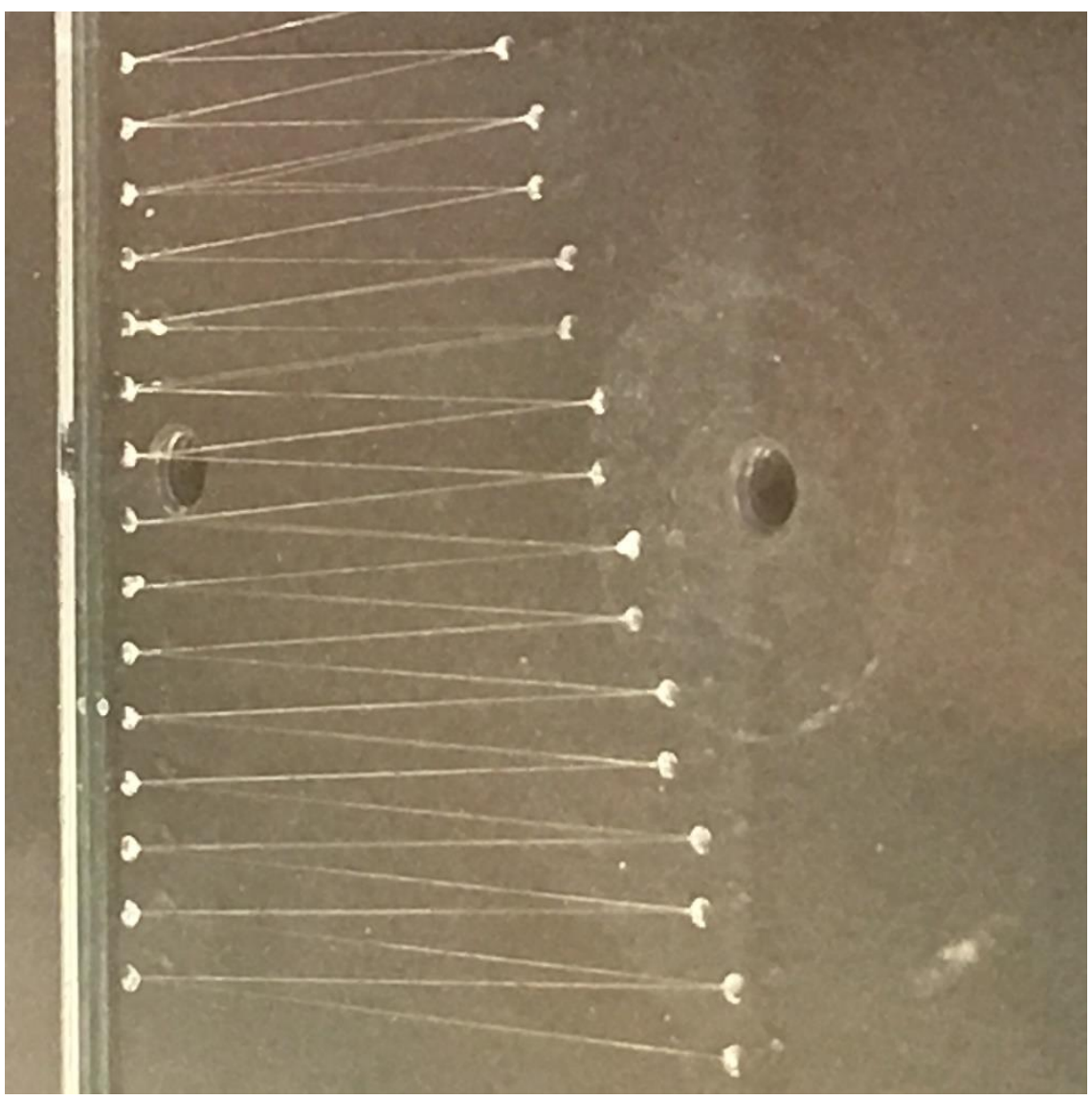

Figure 4. 11 An optical image of PMMA fibers drawn on glass substrate with different dimensions

After completing the above characterization process, a series of experiments were performed under specific conditions to try to produce uniform and repeatable fiber filaments as small as $\sim 300 \mathrm{~nm}$ (Table 4.2 \& Figure 4.11). As mentioned before, the leading diameter of each fiber was at a location of $200 \mu \mathrm{m}$ away from the initiation drawing point and the terminating diameter was located $200 \mu \mathrm{m}$ away from the termination drawing point. The middle measurement location was at the center length of each fiber. In general, the 
Table 4. 2 Diameters of PMMA fibers at predetermined conditions (weight concentration 19\%, dispensing time $0.02 \mathrm{~s}$, needle Size 32 gauge, feed rates $15 \mathrm{~mm} / \mathrm{s}$ ).

\begin{tabular}{lllllll}
\hline Fiber \# & $\begin{array}{l}\text { Length } \\
(\mathbf{m m})\end{array}$ & $\begin{array}{l}\text { Leading } \\
\text { Diameter } \\
(\boldsymbol{\mu m})\end{array}$ & $\begin{array}{l}\text { Middle } \\
\text { Diameter } \\
(\boldsymbol{\mu m})\end{array}$ & $\begin{array}{l}\text { Terminating } \\
\text { Diameter } \\
(\boldsymbol{\mu}) \boldsymbol{m})\end{array}$ & $\begin{array}{l}\text { Average } \\
\text { Diameter } \\
(\boldsymbol{\mu} m)\end{array}$ & $\begin{array}{l}\text { Standard } \\
\text { Deviation } \\
(\boldsymbol{\mu}) \boldsymbol{m})\end{array}$ \\
\hline 1 & 22 & 0.870 & 0.384 & 0.760 & 0.671 & 0.208 \\
2 & 22 & 0.612 & 0.301 & 0.365 & 0.426 & 0.134 \\
3 & 24 & 0.416 & 0.431 & 0.806 & 0.551 & 0.180 \\
4 & 24 & 0.487 & 0.378 & 0.904 & 0.590 & 0.227 \\
5 & 26 & 0.339 & 0.256 & 0.872 & 0.489 & 0.273 \\
6 & 26 & 0.328 & 0.332 & 0.281 & 0.314 & 0.023 \\
7 & 28 & 0.453 & 0.461 & 0.353 & 0.422 & 0.049 \\
8 & 28 & 0.284 & 0.316 & 0.450 & 0.350 & 0.072 \\
9 & 30 & 0.335 & 0.405 & 0.409 & 0.383 & 0.034 \\
10 & 30 & 0.285 & 0.295 & 0.279 & 0.286 & 0.006 \\
\hline
\end{tabular}

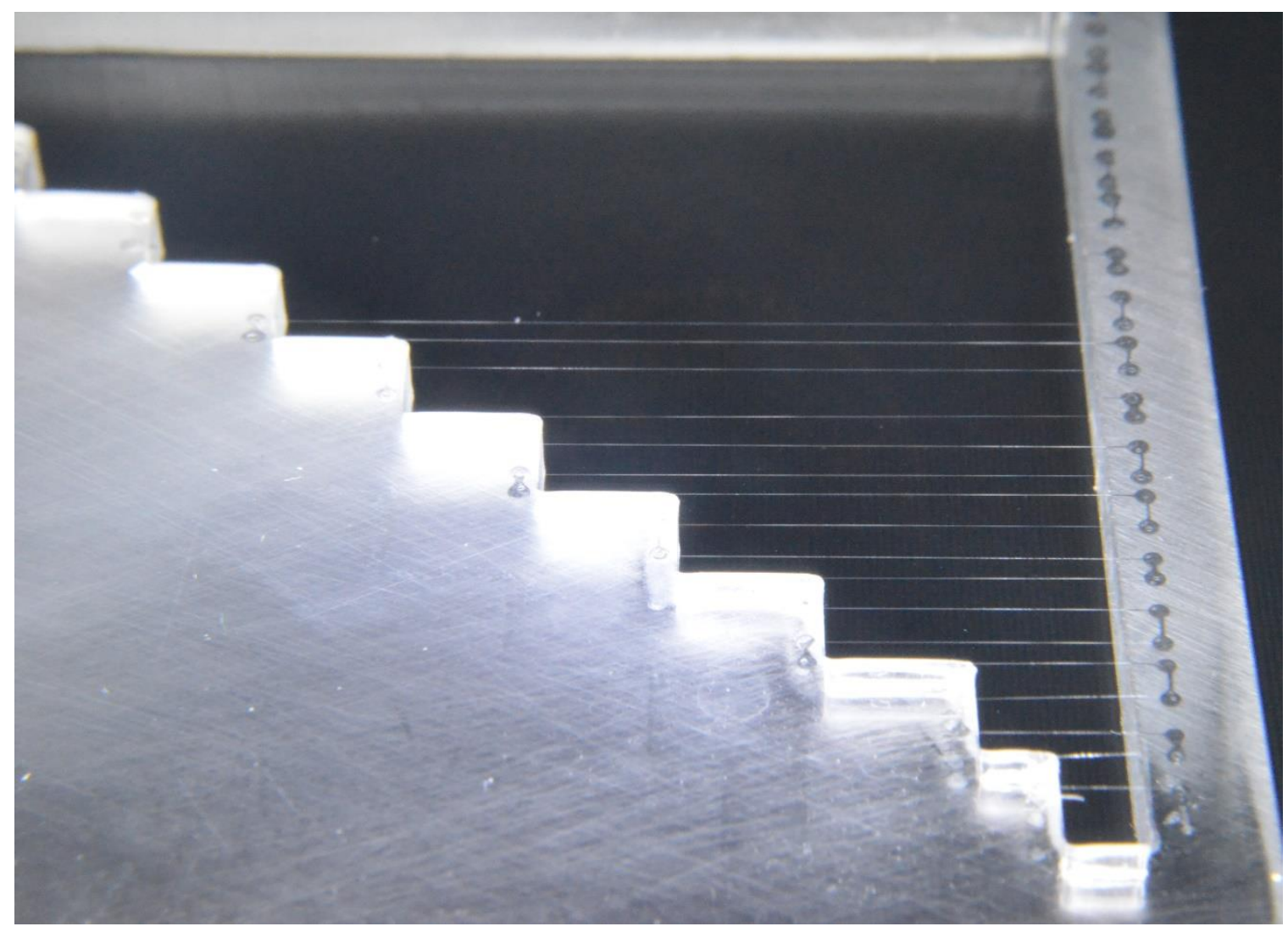

Figure 4. 12 PMMA fibers drawn on the hollow substrate with different lengths 


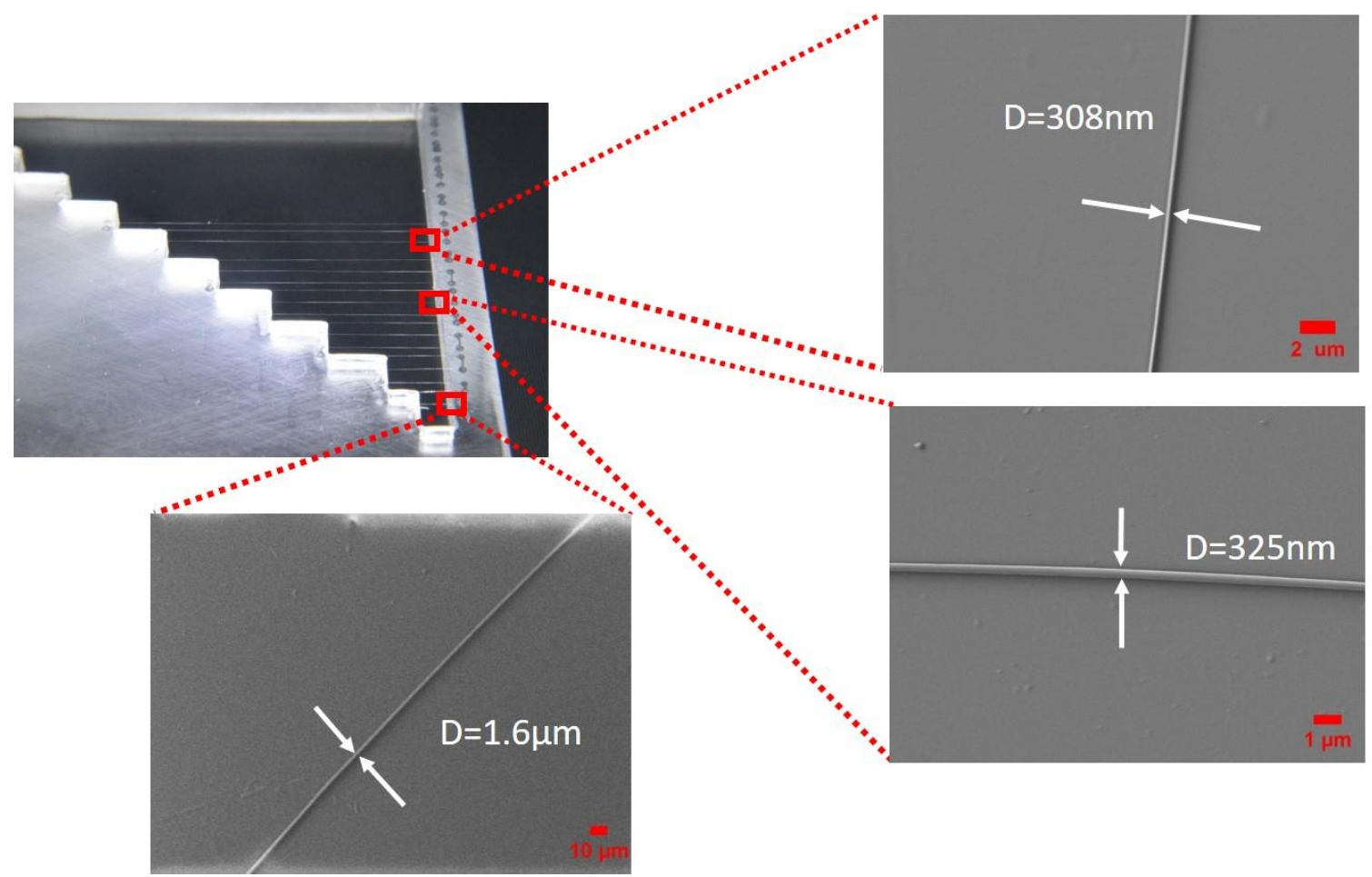

Figure 4. 13 Optical (upper left) and SEM (the three insets) images of PMMA fibers with specific dimensions on the hollow acrylic substrate

Table 4. 3 Optimized diameters of suspended PMMA fibers onto hollow Substrate at predetermined condition (weight concentration 20\%, dispensing time $0.02 \mathrm{~s}$, needle size 32 gauge, feed rates 15 $\mathrm{mm} / \mathrm{s})$.

\begin{tabular}{lllllll}
\hline Fiber \# & $\begin{array}{l}\text { Length } \\
(\mathbf{m m})\end{array}$ & $\begin{array}{l}\text { Leading } \\
\text { Diameter } \\
(\boldsymbol{\mu m})\end{array}$ & $\begin{array}{l}\text { Middle } \\
\text { Diameter } \\
(\boldsymbol{\mu m})\end{array}$ & $\begin{array}{l}\text { Terminating } \\
\text { Diameter } \\
(\boldsymbol{\mu}) \boldsymbol{m})\end{array}$ & $\begin{array}{l}\text { Average } \\
\text { Diameter } \\
(\boldsymbol{\mu} m)\end{array}$ & $\begin{array}{l}\text { Standard } \\
\text { Deviation } \\
(\boldsymbol{\mu}) \boldsymbol{m})\end{array}$ \\
\hline 1 & 2.5 & 1.59 & 1.331 & 1.373 & 1.431 & 0.113 \\
2 & 2.5 & 1.272 & 1.483 & 1.434 & 1.396 & 0.090 \\
3 & 5 & 0.953 & 0.876 & 0.944 & 0.924 & 0.034 \\
4 & 5 & 0.975 & 1.012 & 0.907 & 0.965 & 0.043 \\
5 & 10 & 0.790 & 0.895 & 0.920 & 0.868 & 0.056 \\
6 & 10 & 0.935 & 0.923 & 0.863 & 0.907 & 0.032 \\
7 & 15 & 0.759 & 0.653 & 0.701 & 0.704 & 0.043 \\
8 & 15 & 0.617 & 0.681 & 0.747 & 0.682 & 0.053 \\
9 & 20 & 0.664 & 0.623 & 0.683 & 0.657 & 0.025 \\
10 & 20 & 0.574 & 0.543 & 0.591 & 0.570 & 0.020 \\
11 & 25 & 0.463 & 0.377 & 0.404 & 0.415 & 0.036 \\
12 & 25 & 0.471 & 0.407 & 0.317 & 0.398 & 0.063 \\
\hline
\end{tabular}


goal of fabricating fibers with diameters as small as $300 \mathrm{~nm}$ was achieved. Furthermore, the average diameter of the fibers became smaller when its length became larger. Similarly, the repeatability of the process and uniformity of the fibers appeared to increase with fiber length as evidenced by the tendency for the standard deviations to decrease with fiber length. In order to show the repeatability and controllability of this new system, another round of experiments with the same experimental parameter except concentration at $20 \%$ were performed using hollow substrates to draw completely suspended, parallel oriented fibers. With the optimized controlling factors identified, sub-micron scale fibers were obtained as small as $\sim 400 \mathrm{~nm}$ (Table 4.3 \&Figures 4.12 - 4.13) on a hollow substrate without any fiber resting on the main body except two ending droplets.

Usually, it was difficult to control the experimental process to obtain the desired fiber dimensions due to the trade-off between volatility and viscosity of the PMMA solutions. At low volatility and high viscosity, the final fibers became large in diameter; while, the fibers were very fragile and broke easily when the volatility was high and the viscosity was low. For this system, volatility was related to the dispense time, feed rates, needle tip size, and fiber length, while the viscosity and surface tension were dependent on the weight concentration of the PMMA solutions. Fibers from $\sim 300 \mathrm{~nm}$ to $100 \mu \mathrm{m}$ diameter were successfully drawn individually during the optimization process for different combined conditions. It was easy to draw the desired fiber diameters by combining the information attained from the optimization method and empirical prediction equation with the experimental controlling parameters of these additional studies.

In the following group SEM images (), different range diameter fibers were attained for different controlling factors. For example, large diameter fibers were repeatedly drawn 


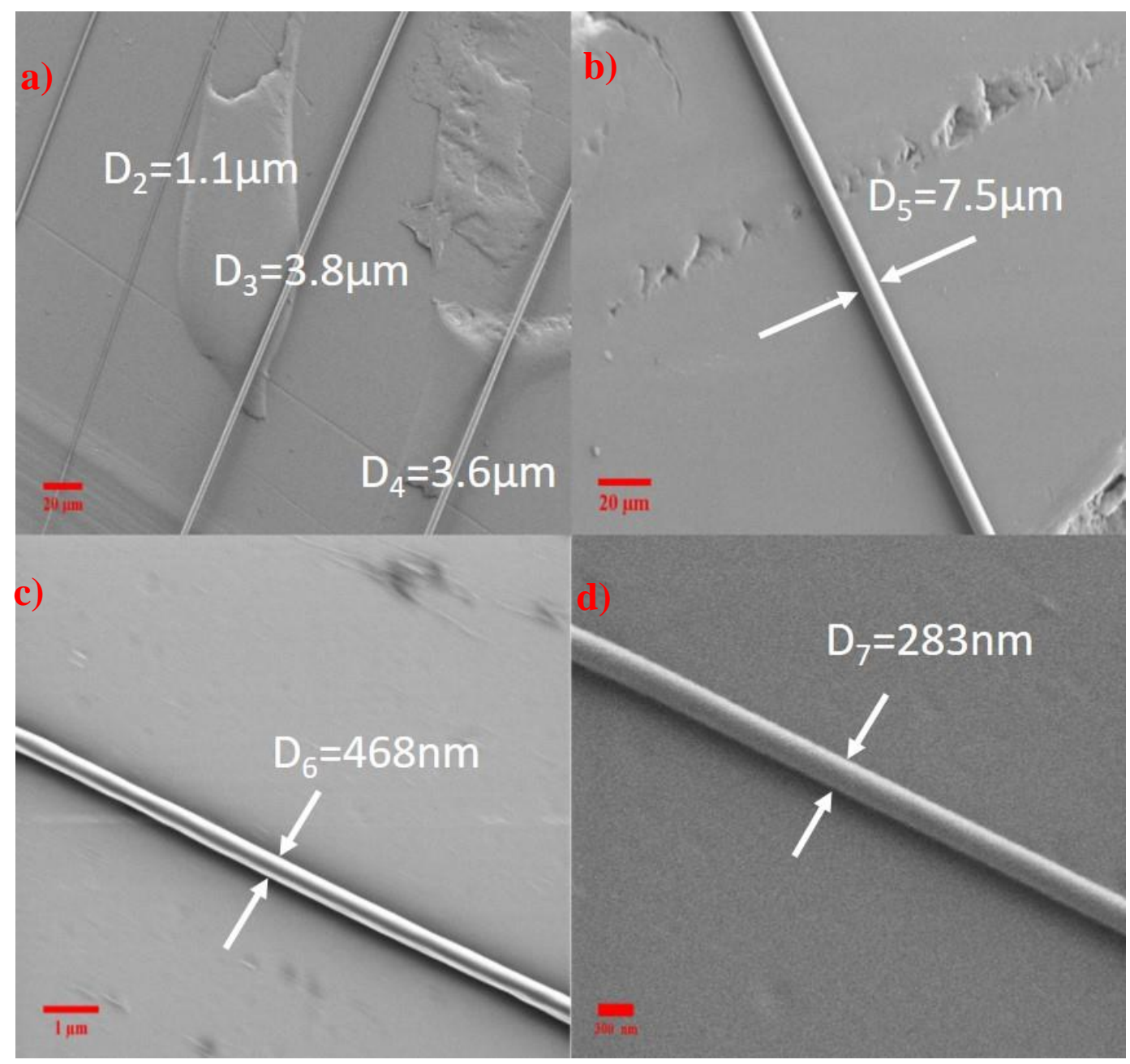

Figure 4. 14 SEM images of PMMA fibers with specific diameter at predetermined conditions $(2.0 \mathrm{KV}$ EHT $)$

(Figure 4.14a \& b) for experiment factors at 24\% concentration, $0.5 s$ dispense time, 32 needle gauge and fiber length ranging from $5 \mathrm{~mm}$ to $40 \mathrm{~mm}$ with the distance of these fibers controlled at around $100 \mu \mathrm{m}$ and $50 \mu \mathrm{m}$ (Figure 4.14a), respectively. By adjusting some significant factors including concentration, feed rate, and dispense time, fibers' diameter can be controlled within $500 \mathrm{~nm}$ (Figure 4.14c). Fiber diameters as small as $280 \mathrm{~nm}$ were drawn via the 3-axis roboti c dispensing system using the optimized controlling factors of 
weight concentration $20 \%$, dispensing time $0.02 \mathrm{~s}$, needle size 32 gauge, feed rates $15 \mathrm{~mm} / \mathrm{s}$ (Figure 4.14d).

\subsubsection{Empirical Prediction Equation Generation}

The data from our previous characterization work was used to generate an empirical prediction equation. First, the dimensionless parameters were calculated using the corresponding controlling parameters and the relationships described above. Then, a linear regression function generated via Matlab [134] was utilized to find the coefficients for each term of variables, which was approximated by a third-order polynomial using the capillary number $(\mathrm{Ca})$, processability parameter $(\mathrm{P})$, aspect ratio $(\Lambda)$, and dispense time $(\mathrm{Dt})$ variables to determine fiber diameter $\left(\mathrm{D}_{\infty}\right)$ :

$$
\begin{gathered}
D_{\infty}=516.201-1.021 \times 10^{4} \mathrm{Dt}+3.3437 \times 10^{4} \mathrm{Dt}^{2}-2.448 \mathrm{Dt}+1.613 \times 10^{6} \mathrm{P}+ \\
\begin{array}{c}
13.623 \mathrm{Ca}-2.575 \times 10^{-1} \mathrm{Ca}^{2}+1.226 \times 10^{-3} \mathrm{Ca}^{3}-8.344 \Lambda+3.742 \times 10^{-2} \Lambda^{2}- \\
4.613 \times 10^{-5} \Lambda^{3}
\end{array}
\end{gathered}
$$

From the above equation, the high order terms of $P$ were excluded due to the negligible effect of these terms on the final diameter of the fiber.

\subsubsection{Minimum Diameter of PMMA Fibers}

To find the minimum diameter of PMMA fibers that could be fabricated, we combined our previous characterization work with theoretical analysis. A genetic algorithm (GA) from Matlab was utilized to solve both constrained and unconstrained optimization problems based on a natural selection process that mimicked biological evolution [135]. This function generated a population of different solutions at each iteration 
and, eventually identified the best solution that optimized Eq. (4-1). In this work, the genetic algorithm was used with the following structure [20]:

- Chromosome: Each chromosome has 50 bits divided into five sets, and each set consists of 10 bits to present one variable to be optimized.

- Fitness: Since GA was a Global optimization approach looking for the global maximum point, we defined the fitness of an individual Chromosome (one solution) as the inverse empirical prediction equation as:

Fitness $=1 / D_{\infty}=1 /\left(516.201-1.021 \times 10^{4} \mathrm{Dt}+3.344 \times 10^{4} \mathrm{Dt}^{2}-2.448 D t^{3}+\right.$ $1.613 \times 10^{6} \mathrm{P}+13.623 \mathrm{Ca}-2.575 \times 10^{-1} \mathrm{Ca}^{2}+1.226 \times 10^{-3} \mathrm{Ca}^{3}-8.344 \Lambda+$ $3.742 \times 10^{-2} \Lambda^{2}-4.613 \times 10^{-5} \Lambda^{3}$ )

Table 4. 4 Optimal controlling parameters

\begin{tabular}{|c|c|}
\hline Controlling Parameters Types & Optimal Controlling Parameters Value \\
\hline Needle Tip Size (Gauge) & 30 \\
\hline Feed Rates $(\mathrm{mm} / \mathrm{s})$ & 300 \\
\hline Dispense Time $(\mathrm{s})$ & 0.02 \\
\hline Polymer Solution Concentration \\
(by Weight)
\end{tabular}

By using this inverse empirical prediction equation, the maximum value of the function was determined in a two thousand times loop that included all the iteration steps of the genetic algorithm. After running the code, the minimum diameter of fiber that could be fabricated with our system using the parameters identified was found to be $183 \mathrm{~nm}$. The 
algorithm outputted the corresponding controlling parameters required to achieve this fiber diameter (Table 4.4), which have been displayed in red font.

\subsubsection{Validation of Empirical Equation}

With the optimal combinations of controlling parameters provided by the genetic algorithm, it was necessary to perform experiments with the robotic dispensing system in order to validate the prediction equation and the algorithm code. To provide statistically viable data, 20 trials were performed at the same presetting of the controlling parameters. It turned out that only two of the 20 runs (refer to red fond in Table 4.4) yielded diameters

Table 4. 5 Performance data of fibers at optimal condition with 15 psi pressure

\begin{tabular}{cccccc}
\hline Fiber \# & $\begin{array}{c}\text { Length } \\
(\mathbf{m m})\end{array}$ & $\begin{array}{c}\text { Average } \\
\text { Diameter } \\
(\boldsymbol{\mu m})\end{array}$ & $\begin{array}{c}\text { Standard } \\
\text { Deviation } \\
(\boldsymbol{\mu})\end{array}$ & $\begin{array}{c}\text { Initiating } \\
\text { Droplet } \\
\text { Diameter } \\
(\boldsymbol{\mu m})\end{array}$ & $\begin{array}{c}\text { Prediction } \\
\text { Error }\end{array}$ \\
\hline 1 & 35 & 4.565 & 0.181 & 946.1 & $2391.41 \%$ \\
2 & 35 & 1.624 & 0.078 & 972.3 & $786.07 \%$ \\
3 & 35 & 1.540 & 0.022 & 909 & $740.23 \%$ \\
4 & 35 & 1.500 & 0.040 & 909.4 & $718.77 \%$ \\
5 & 35 & 1.418 & 0.024 & 905.4 & $673.65 \%$ \\
6 & 35 & 1.350 & 0.044 & 907.7 & $636.91 \%$ \\
7 & 35 & 1.333 & 0.036 & 901 & $627.63 \%$ \\
8 & 35 & 1.270 & 0.048 & 904.8 & $592.89 \%$ \\
9 & 35 & 1.160 & 0.039 & 899.1 & $533.04 \%$ \\
10 & 35 & 0.961 & 0.013 & 879.8 & $424.19 \%$ \\
11 & 35 & 0.940 & 0.021 & 895.7 & $413.18 \%$ \\
12 & 35 & 0.921 & 0.022 & 893.1 & $402.54 \%$ \\
13 & 35 & 0.860 & 0.026 & 881.7 & $369.07 \%$ \\
14 & 35 & 0.578 & 0.017 & 858.3 & $215.48 \%$ \\
15 & 35 & 0.563 & 0.018 & 849.9 & $207.42 \%$ \\
16 & 35 & 0.343 & 0.012 & 837.2 & $87.31 \%$ \\
17 & 35 & 0.319 & 0.024 & 830.4 & $73.83 \%$ \\
18 & 35 & 0.198 & 0.007 & 807.2 & $8.29 \%$ \\
19 & 35 & 0.161 & 0.006 & 772.6 & $12.05 \%$ \\
20 & 35 & 0.122 & 0.009 & 760.9 & $33.46 \%$ \\
\hline
\end{tabular}


in the approximate predicted range of $183 \mathrm{~nm}$. The range was quantified here by calculating the prediction errors and comparing to the experimental value:

$$
\text { Prediction Error }=\frac{\mid \text { experimental value-predicted value } \mid}{\text { predicted value }}
$$

When the corresponding prediction error was less than $15 \%$, the experimental value was considered to be located in the prediction range.

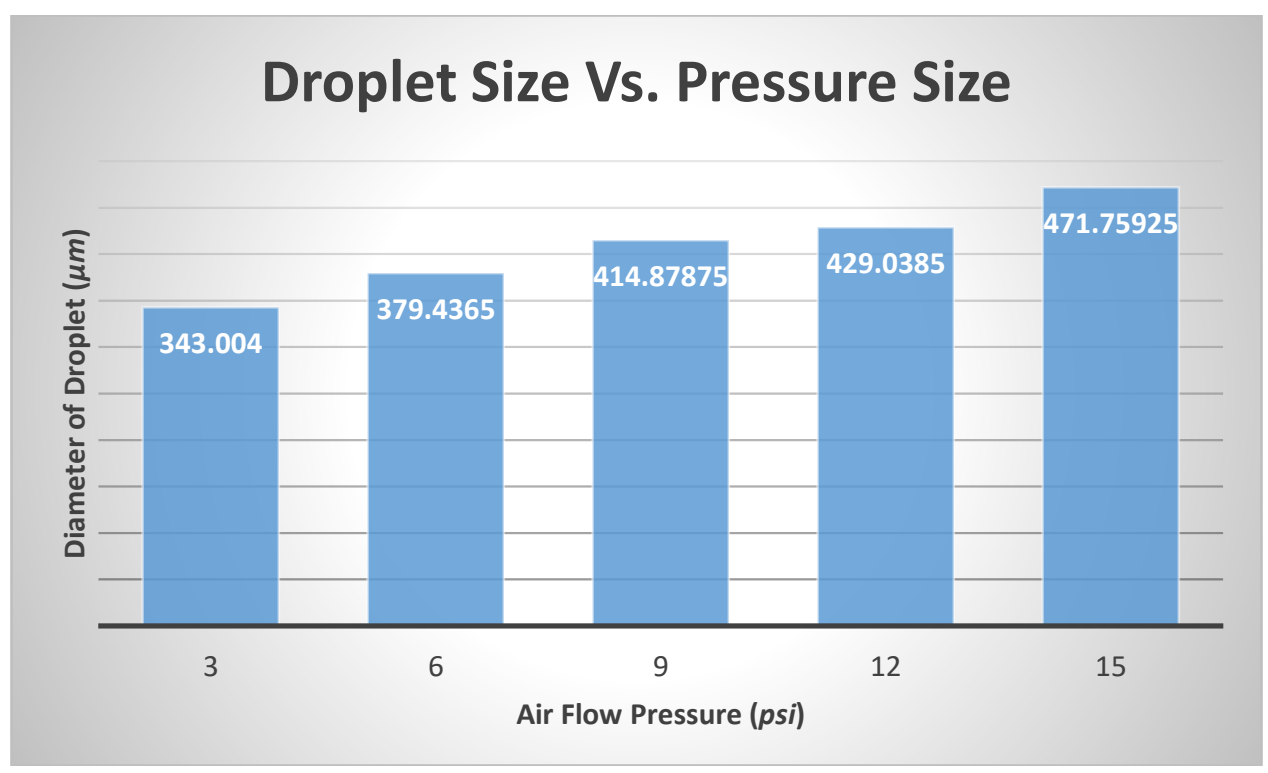

Figure 4. 15 Effect of pressure on droplet size

Until now, only one controlling parameter of this new system was not utilized to characterize the direct-write process, the flow of air pressure applied onto the polymer solutions, which could range from 0 to $15 \mathrm{psi}$. In all of the previous experiments, the maximum value, $15 \mathrm{psi}$, was used to expel polymer solutions at the beginning of each drawing. It was found in fact (Table 4.5) that the initiating droplet size has a direct affect on the final diameter of the fibers. Since the initiating droplet was dependent on the air 
flow pressure, additional experiments were performed to measure the droplet size as the polymer droplet was discharged from the tip of the syringe needle for varying pressure levels using a USB- microscope and image processing software (Image J) (Table 4.6). It was revealed that droplet size was directly proportional to the size of the air pressure (Figure 4.15) with an optimal air pressure of $3 p s i$ since the largest number of fibers were successfully fabricated within the predicted range at this pressure (Figure 4.16).

Table 4. 5 Performance data of fibers at optimum condition at 3 psi pressure

\begin{tabular}{cccccccc}
\hline Fiber \# & $\begin{array}{c}\text { Length } \\
(\boldsymbol{m m})\end{array}$ & $\begin{array}{c}\text { Leading } \\
\text { Diameter } \\
(\boldsymbol{\mu m})\end{array}$ & $\begin{array}{c}\text { Middle } \\
\text { Diameter } \\
(\boldsymbol{\mu m})\end{array}$ & $\begin{array}{c}\text { Terminating } \\
\text { Diameter } \\
(\boldsymbol{\mu m})\end{array}$ & $\begin{array}{c}\text { Average } \\
\text { Diameter } \\
(\boldsymbol{\mu m})\end{array}$ & $\begin{array}{c}\text { Standard } \\
\text { Deviation } \\
(\boldsymbol{\mu m})\end{array}$ & $\begin{array}{c}\text { Prediction } \\
\text { Error }\end{array}$ \\
\hline 1 & 35 & 0.6589 & 0.6332 & 0.6008 & 0.631 & 0.024 & $244.33 \%$ \\
2 & 35 & 0.375 & 0.3873 & 0.3915 & 0.385 & 0.007 & $109.89 \%$ \\
3 & 35 & 0.3522 & 0.3366 & 0.3353 & 0.341 & 0.008 & $86.29 \%$ \\
4 & 35 & 0.3383 & 0.306 & 0.3185 & 0.321 & 0.013 & $75.14 \%$ \\
5 & 35 & 0.2755 & 0.2758 & 0.2696 & 0.274 & 0.003 & $49.33 \%$ \\
6 & 35 & 0.2514 & 0.2686 & 0.2734 & 0.264 & 0.009 & $44.33 \%$ \\
7 & 35 & 0.2797 & 0.2549 & 0.2523 & 0.262 & 0.012 & $43.14 \%$ \\
9 & 35 & 0.2652 & 0.2301 & 0.2731 & 0.256 & 0.019 & $39.78 \%$ \\
10 & 35 & 0.259 & 0.2321 & 0.2291 & 0.240 & 0.013 & $31.01 \%$ \\
11 & 35 & 0.2283 & 0.2236 & 0.2247 & 0.226 & 0.002 & $23.08 \%$ \\
12 & 35 & 0.2109 & 0.2079 & 0.1893 & 0.203 & 0.010 & $10.62 \%$ \\
13 & 35 & 0.1996 & 0.1921 & 0.2099 & 0.201 & 0.007 & $9.44 \%$ \\
14 & 35 & 0.2096 & 0.1908 & 0.1903 & 0.197 & 0.009 & $7.45 \%$ \\
15 & 35 & 0.219 & 0.1824 & 0.1876 & 0.196 & 0.016 & $7.14 \%$ \\
16 & 35 & 0.212 & 0.1904 & 0.183 & 0.195 & 0.012 & $6.49 \%$ \\
17 & 35 & 0.2112 & 0.178 & 0.1713 & 0.187 & 0.017 & $1.96 \%$ \\
18 & 35 & 0.2049 & 0.1753 & 0.1795 & 0.187 & 0.013 & $1.81 \%$ \\
19 & 35 & 0.18 & 0.1904 & 0.178 & 0.183 & 0.005 & $0.24 \%$ \\
20 & 35 & 0.1932 & 0.1754 & 0.1696 & 0.179 & 0.010 & $2.10 \%$ \\
\hline
\end{tabular}

The key component of the genetic function was the outer iteration loop applied to determine the minimum value by comparing all of the different initial characterization results over two thousand times. Based on the above algorithm, the minimum predicted fiber diameter was determined to be $183 \mathrm{~nm}$. Using the predicted process parameters, the 


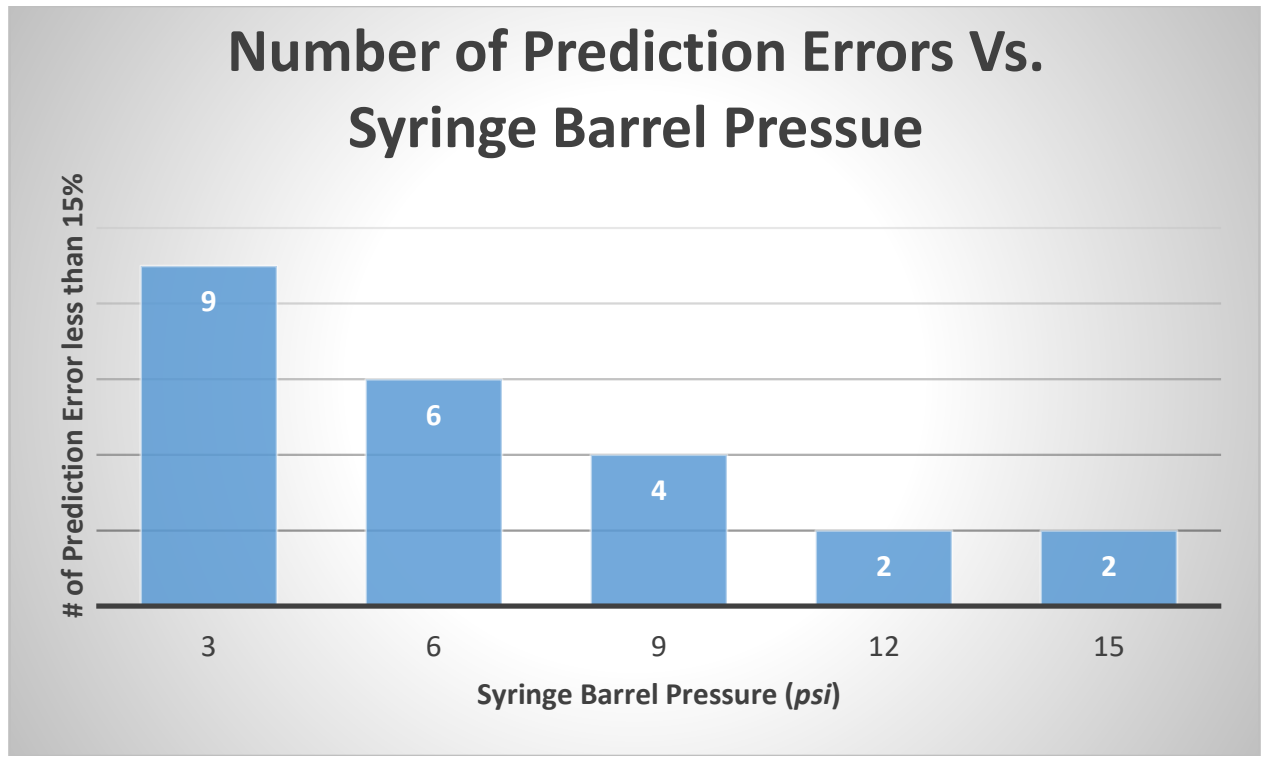

Figure 4. 16 Number of prediction errors less than $15 \%$ at different pressure

robotic dispensing system was utilized to verify the theoretical analysis data by running the experiment 20 times at prescribed conditions. Comparing the initiating droplets at different fiber diameter, it is good to know that the air flow pressure is another important controlling factor affecting the performance of fiber formation. The diameters of the polymer droplet discharged from the syringe needle tip are listed in Figure 4.15 at different air flow pressure. The polymer dropet discharge diameter is determined by measuring the actual size of images taken from a USB-microscope. Therefore, in the last step of optimization, experiments were run 20 times over a range of air flow pressures, from 3 Psi to 15 Psi in 3 Psi increments.

\subsection{Fabrication of 3-D Micro/Nano Fluidic Electroporation Devices}

A total of 18 micro/nanofluidic PDMS devices were batch fabricated with a gap distance and chamber depth of $10.7 \pm 0.51 \mu \mathrm{m}$ and $30.5 \pm 1.42 \mu \mathrm{m}$, respectively, (Table 4 . 
6). Although the original thickness of the dry film resists (DFRs) was $20 \mu m$, the actual depth of the micro-chambers (with two layers of DFRs) was found to be $30.5 \mu \mathrm{m}$ due to thinning of the DFR material during the post exposure bake and development processes, together with the merging of the 2 layers during the film-to-film bonding step at elevated temperatures. Overall, for the $18 \mathrm{micro} /$ nanofluidic devices fabricated, the physical dimensions of the micro-chambers were controlled within $7 \%$ of the design value (Table 4.8). For the actual dimensions of the channels, the accuracy of the channels' diameters for the 6 fluidic devices fabricated for each desired channel dimension $(1000 \pm 9 \mathrm{~nm}, 500 \pm 23$ $\mathrm{nm}$ and $300 \pm 17 \mathrm{~nm}$ ) were within $6 \%$ of the design for the final PDMS devices (Table 4.8). It has been proven that all the trial data (total 18 fibers) based on characterization results at different scales ranging from $1 \mu \mathrm{m}$ to $500 \mathrm{~nm}$, and $300 \mathrm{~nm}$ located within $20 \%$ of design value for each scale (Table 4.8). In detail, the experimental data of $1 \mu \mathrm{m}$ fiber case were controlled within $12 \%$ of design value; the experimental data of $500 \mathrm{~nm}$ fiber case were controlled within $20 \%$ of design value; the experimental data of $300 \mathrm{~nm}$ fiber case were controlled within $8 \%$ of design value. Therefore, it is possible to draw repeatable and controllable micro/submicron/nanofibers at prescribed experimental conditions via this 3-axis robotic dispensing system.

It was noted that the dimension of the channel imbedded in PDMS was slightly smaller than the original fiber diameter. This reduction in the channel dimension may have been due to the acetone removal process since it has been reported that PDMS swells isotropically in an acetone bath [19]. Because of this behavior, it was anticipated that the isotropic swelling of the PDMS would push against the solvating PMMA fibers, thereby expelling the PMMA out of the channel. Subsequently, when the acetone was removed, 
the PDMS may not have returned to its original dimension due to the presence of residual acetone or induction of internal stresses in the PDMS, which led to smaller channel diameters. As a result, the final PDMS channels were determined to decrease by $10.1 \%$, $13.1 \%$ and $1.9 \%$ compared to the original fiber diameter for the $1000 \mathrm{~nm}, 500 \mathrm{~nm}$ and 300 $\mathrm{nm}$ designed channels, respectively (Table 4.8). The diameter change for the $300 \mathrm{~nm}$ from fiber to PDMS channel was not as significant as the $1000 \mathrm{~nm}$ and $500 \mathrm{~nm}$ because sonication time for $300 \mathrm{~nm}$ substrate (much thinner PMMA fiber embedded in this substrate) was shorter than other two substrates.

Table 4. 6 Comparison between the design and actual dimensions achieved for the PDMS microchambers for all 18 micro/nanofluidic PDMS devices.

\begin{tabular}{|c|c|c|c|}
\cline { 2 - 4 } \multicolumn{1}{c|}{} & Design Spec. $(\mu \mathrm{m})$ & $\begin{array}{c}\text { Actual Dimension in PDMS } \\
\text { Mean } \pm \text { std dev }(\mu \mathrm{m})\end{array}$ & $\begin{array}{c}\% \text { Diff. } \\
\text { (PDMS - Design) }\end{array}$ \\
\hline Gap Distance & 10 & $10.7 \pm 0.51$ & $7 \%$ \\
\hline $\begin{array}{c}\text { Depth of the Micro } \\
\text { Chambers }\end{array}$ & 30 & $30.5 \pm 1.42$ & $2 \%$ \\
\hline
\end{tabular}

Table 4. 7 Comparison of fiber diameters to actual channel diameters for a total of 18 devices with 6 devices per size group.

\begin{tabular}{|c|c|c|c|}
\hline $\begin{array}{c}\text { Design Dimension of } \\
\text { Channel }(\mathrm{nm})\end{array}$ & $\begin{array}{c}\text { FIBER DIAMETER } \\
\text { Mean } \pm \text { std dev (nm) }\end{array}$ & $\begin{array}{c}\text { PDMS } \\
\text { Mean } \pm \text { std dev (nm) }\end{array}$ & $\begin{array}{c}\% \text { Diff. } \\
\text { (PDMS - Design) }\end{array}$ \\
\hline 1000 & $1122 \pm 13$ & $1009 \pm 33$ & $<1 \%$ \\
\hline 500 & $602 \pm 24$ & $523 \pm 7$ & $<5 \%$ \\
\hline 300 & $323 \pm 9$ & $317 \pm 6$ & $<6 \%$ \\
\hline
\end{tabular}

As mentioned in the Methods section, micro/nanofibers were directly drawn on the DFR molds for the microchambers. The operating parameters for writing the $1000 \mathrm{~nm}, 500$ 
$\mathrm{nm}$ and $300 \mathrm{~nm}$ fibers have been presented in Table 4.9 below. Please note that for each distinct fiber diameter, a range of values were identified since multiple operating parameters could be modified simultaneously or individually to control the fiber diameter. This was anticipated since it is logical that a solution of $21 \%$ polymer concentration required a different feed rate, dispense time, needle tip and syringe pressure than a $19 \%$ or $20 \%$ polymer concentration.

To illustrate the capability of the system to precisely control fiber location on the DFR substrates, two microfibers were drawn on the DFR molded micro-chambers (Figure 4.17). As mentioned previously, individual, highly-oriented, predetermined fibers of 1000 $\mathrm{nm}, 500 \mathrm{~nm}$ and $300 \mathrm{~nm}$ were drawn on the DFR molded micro-chambers, respectively

Table 4. 8 Direct-Write Process Optimized Parameters for Three Different Dimensions' Fibers

\begin{tabular}{|c|c|}
\hline Diameter of Design Fibers & Direct Write Process Parameters \\
\hline \multirow{4}{*}{$1000 \mathrm{~nm}$ Fibers } & Feed Rate $(10-15 \mathrm{~mm} / \mathrm{s})$ \\
\cline { 2 - 2 } & Polymer Solutions Concentration $(20-21 \%)$ \\
\cline { 2 - 2 } & Dispensing Time $(0.02-0.1 \mathrm{~s})$ \\
\cline { 2 - 2 } & Syringe Pressure $(10-15 \mathrm{psi})$ \\
\hline \multirow{5}{*}{$\mathbf{5 0 0} \mathbf{n m}$ Fibers } & Needle Tip Size $(25-32 \mathrm{gauge})$ \\
\cline { 2 - 2 } & Feed Rate $(10-15 \mathrm{~mm} / \mathrm{s})$ \\
\cline { 2 - 2 } & Polymer Solutions Concentration $(19-20 \%)$ \\
\cline { 2 - 2 } & Dispensing Time $(0.02-0.1 \mathrm{~s})$ \\
\hline \multirow{3}{*}{$\mathbf{2 5 0} \mathbf{n m}$ Fibers } & Syringe Pressure $(8-12 \mathrm{psi})$ \\
\cline { 2 - 2 } & Needle Tip Size $(30-32 \mathrm{gauge})$ \\
\cline { 2 - 2 } & Feed Rate $(10-15 \mathrm{~mm} / \mathrm{s})$ \\
\hline & Polymer Solutions Concentration $(19 \%)$ \\
\cline { 2 - 2 } & Dispensing Time $(0.02 \mathrm{~s})$ \\
\hline
\end{tabular}




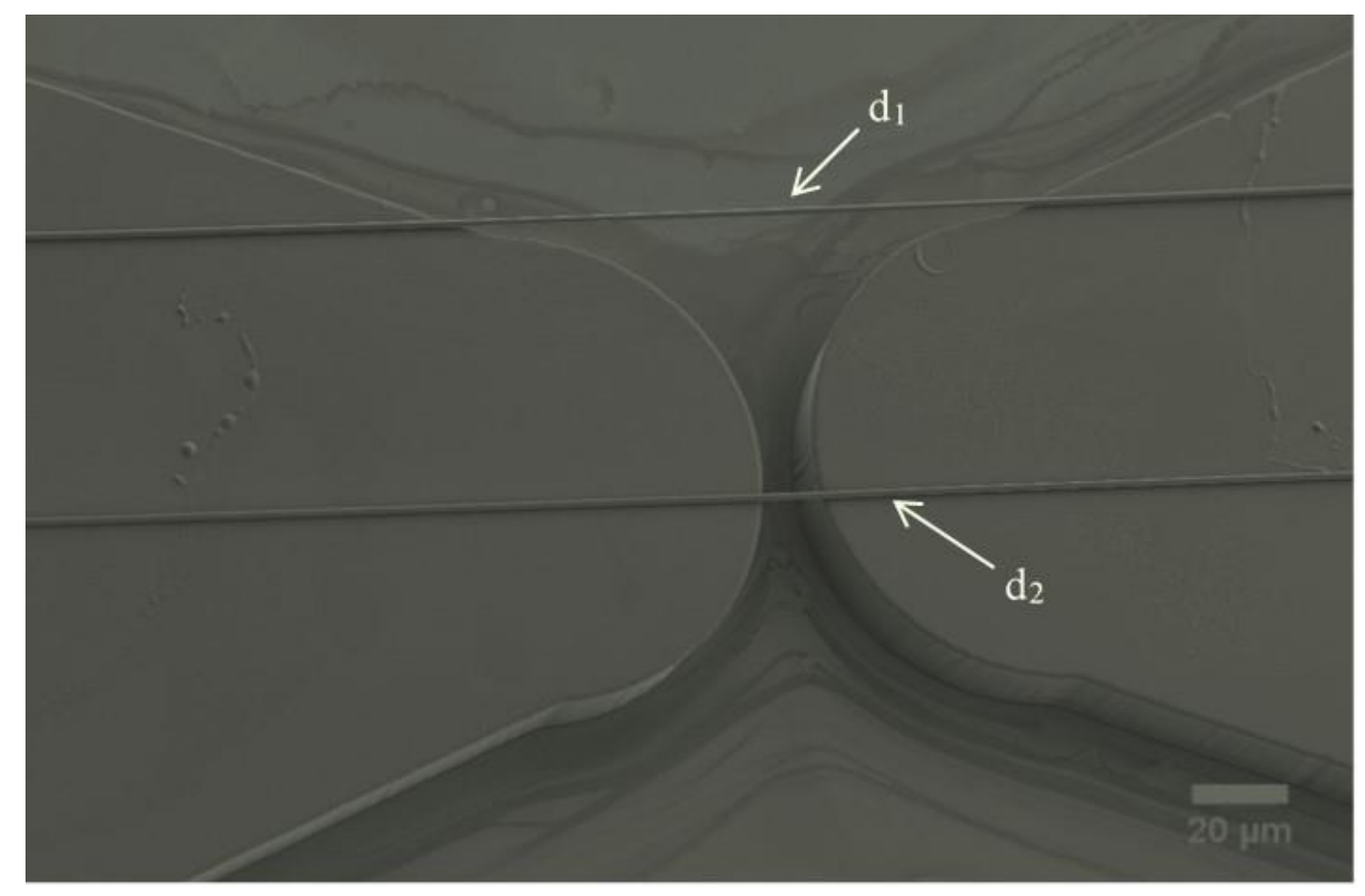

Figure 4. 17 SEM image of the DFRs mold on a glass substrate with PMMA microscale fibers (Top fiber: $\mathrm{d} 1=1.96 \mu \mathrm{m} \&$ Bottom fiber: $\mathrm{d} 2=1.74 \mu \mathrm{m}$ ).

(Figures 4.18-20). After the fibers were drawn on the DFR, the top and bottom substrates containing a layer of DFR were aligned with high precision (within $\leq 2 \mu \mathrm{m}$ tolerance) and bonded to achieve film-to-film bonding and produce the reverse mold of the micro/nanofluidic electroporation PDMS device (Figure 4.21). The micro/nanofibers were sandwiched between these two DFRs, which later acted as the sacrificial structure for the creation of the micro/nanochannel of the PDMS device.

As mentioned in the Methods section, PDMS was poured over the sacrificial DFR microchamber and PMMA fiber molds to produce the PDMS microfluidic platforms (Figure 4. 22a - Figure 4. 24a) for each channel dimension $(\sim 1000 \mathrm{~nm}, \sim 500 \mathrm{~nm}, \sim 300$ $n m$,), respectively. In viewing the images in Figures 4.18-4.20 to Figures 4.22-4.24, the fiber seen in the middle of the DFR was only viewed in the middle "dam" regions after 
bonding since the PMMA fiber in the other regions on the DFR become engulfed/ covered by the second layer DFR.

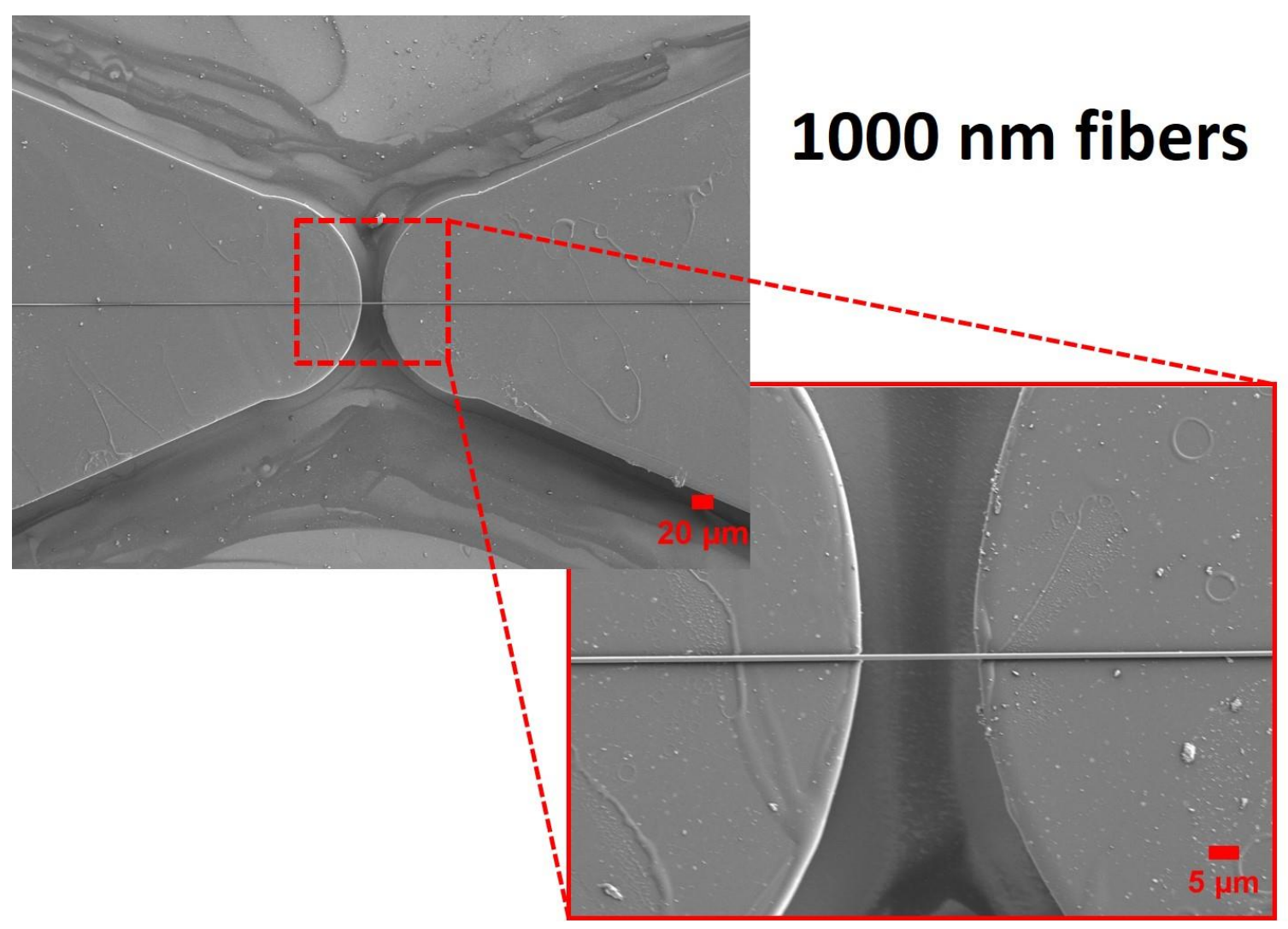

Figure 4. 18 SEM image of the dry film resist mold on a glass substrate with a microscale PMMA fiber at $\sim 1 \mu \mathrm{m}$

Some issues including misalignment, thin fiber written on the right location of the substrate, and the PDMS substrate bonding with glass had been alleviated during PDMS microfluidic platforms fabrication. For drawing thinner fibers on the substrate, it was difficult to repeat the result within $20 \%$ of design value at around $300 \mathrm{~nm}$ since solvent evaporation changed the polymer solutions concentration over time. One solution was to purge the old solution out of the syringe needle before drawing a new fiber. Another misalignment issue occurred when two substrates were bonding together. As mentioned 
before, a new film-to-film bonding and alignment equipment was built to address the issue. Vacuum was used to fix the positions of two substrate before alignment because it was an irreversible process once the fiber was sandwiched by the two substrates. And long working distance was achieved by the stereoscope. High precision alignment (less than 2 $\mu m$ gap) was guaranteed by using a 3 -axis stage and the $63 \mathrm{X}$ stereoscope. Initial PDMS substrate bonding with glass failed several times due to some reasons: 1) the glass or PDMS substrate was not clean enough with organic solvents, 2) long time oxygen plasma treatment, 3) without heat or light force treatment after plasma. As describe in the last

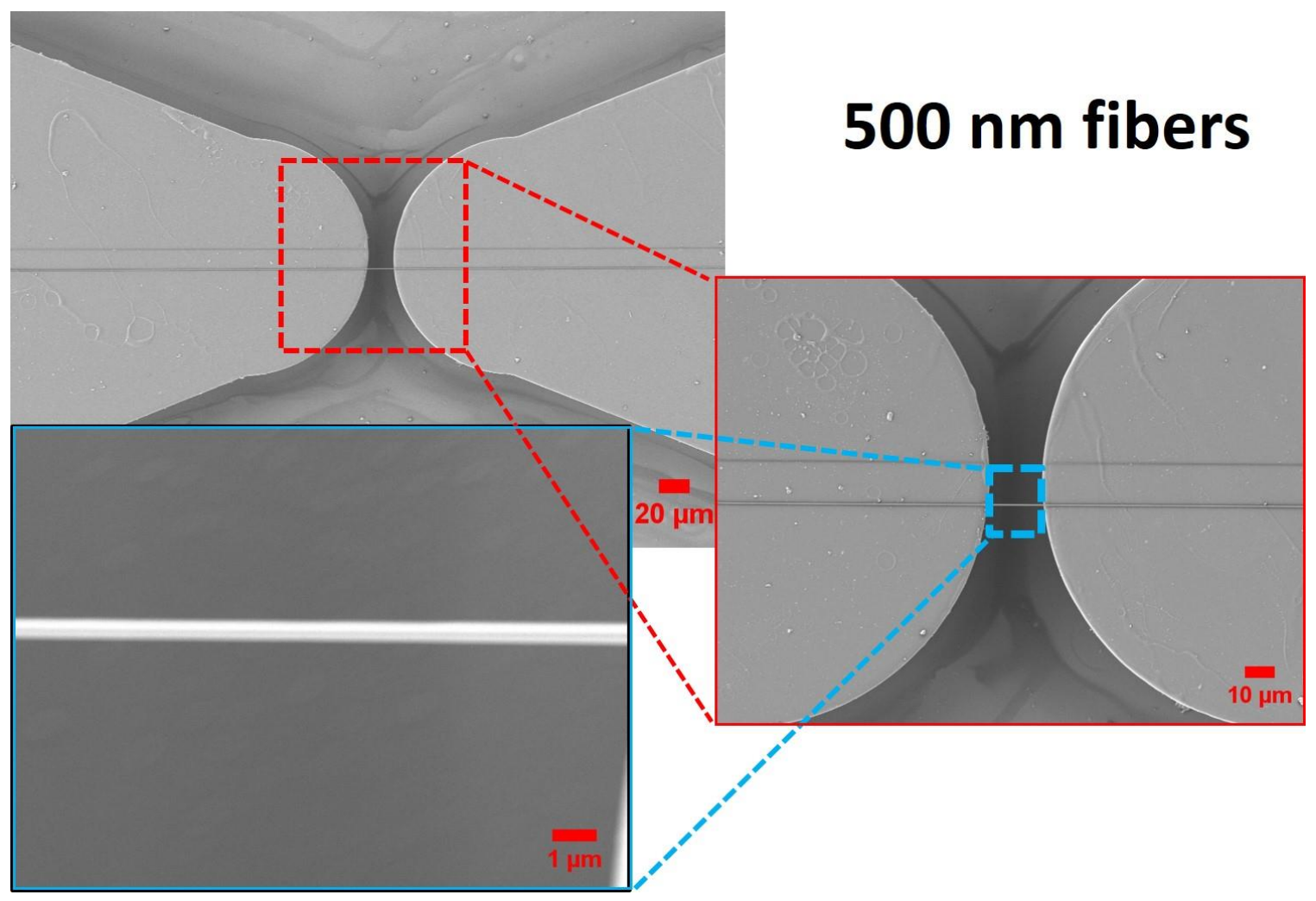

Figure 4. 19 SEM image of the dry film resist mold on a glass substrate with a sub-microscale PMMA fiber at $\sim 500 \mathrm{~nm}$ 


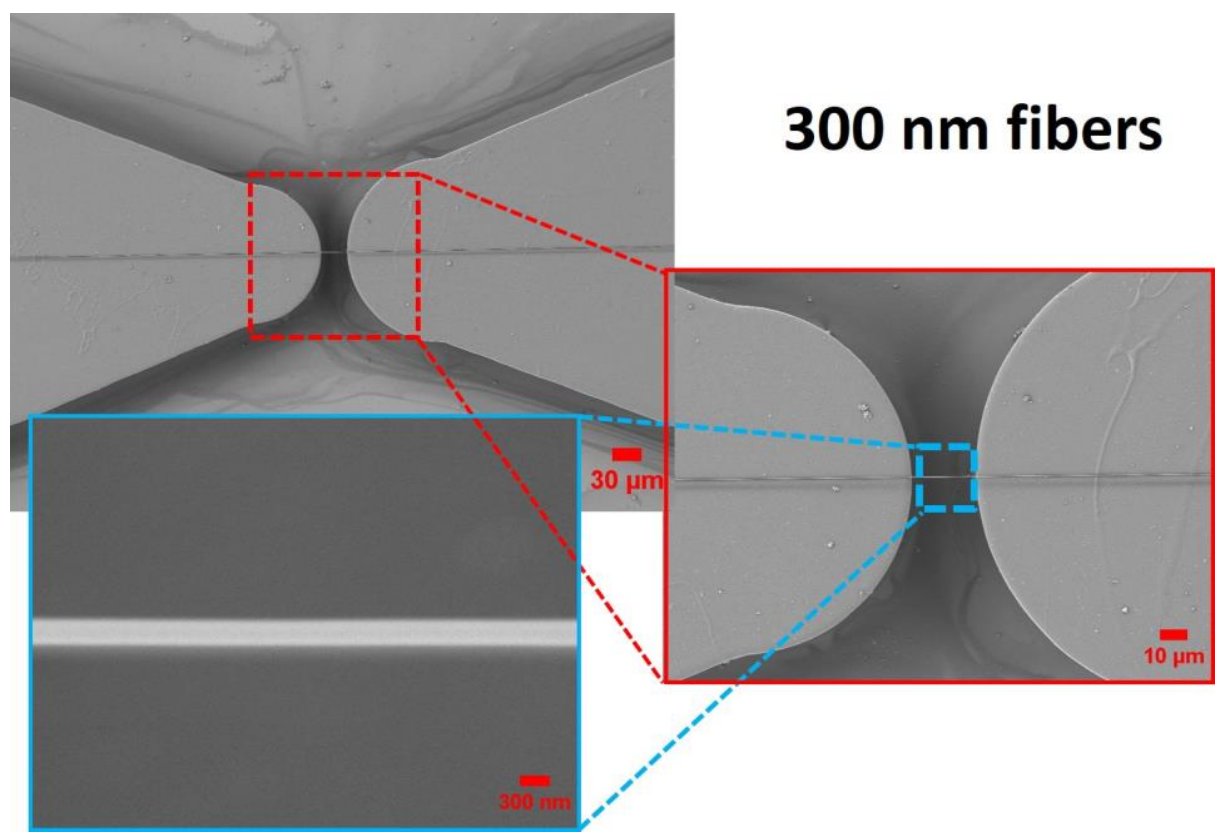

Figure 4. 20 SEM image of the dry film resist mold on a glass substrate with a nanoscale PMMA fiber at $\sim 300 \mathrm{~nm}$

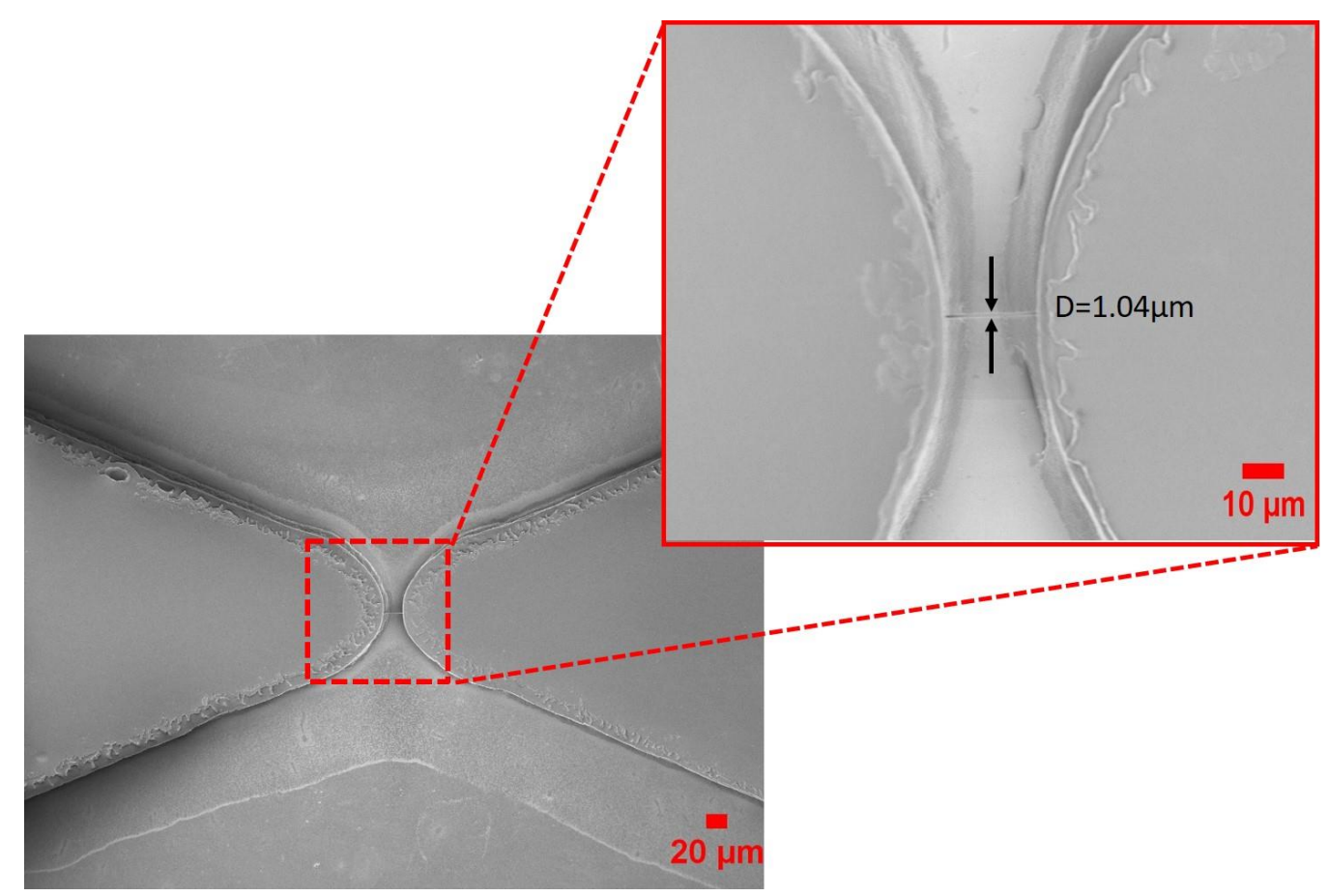

Figure 4. 21 SEM image of two layer dry film resist mold on a glass substrate sandwiching a microscale PMMA fiber 


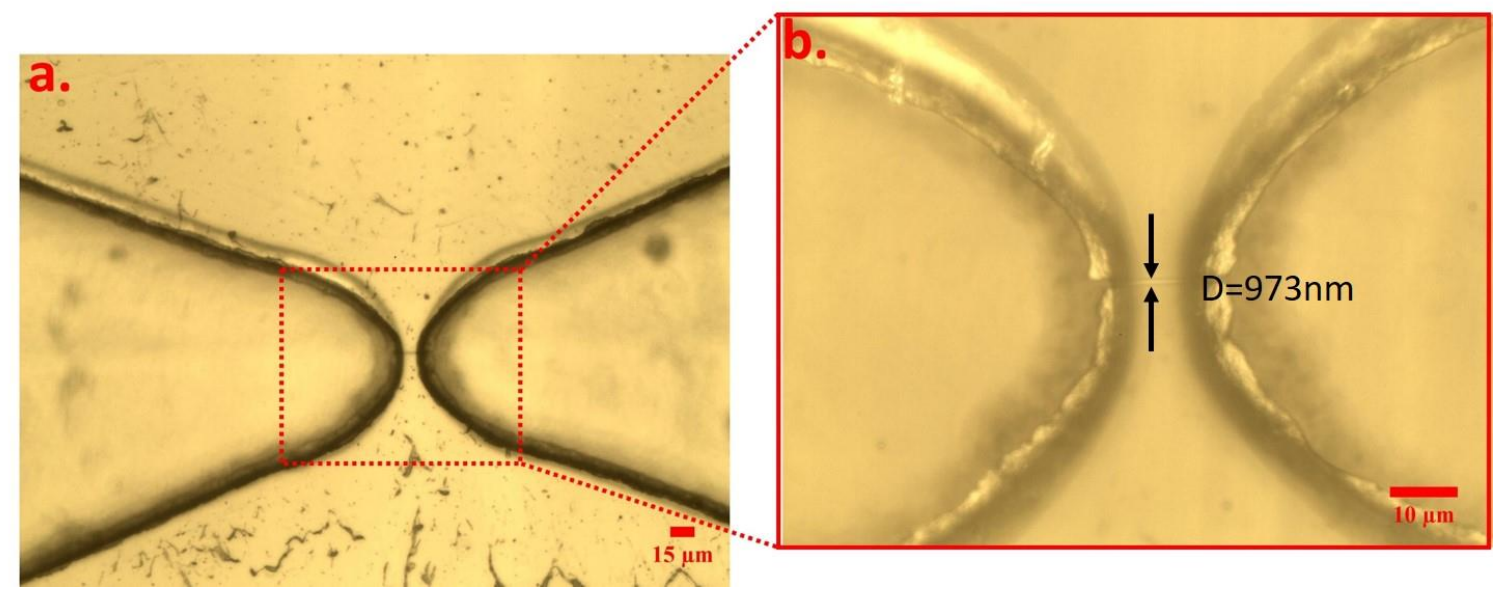

Figure 4. 22 a) Optical image of the micron channel $(\sim 973 \mathrm{~nm})$ embedded within the PDMS "Dam", b) enlarged view of middle channel

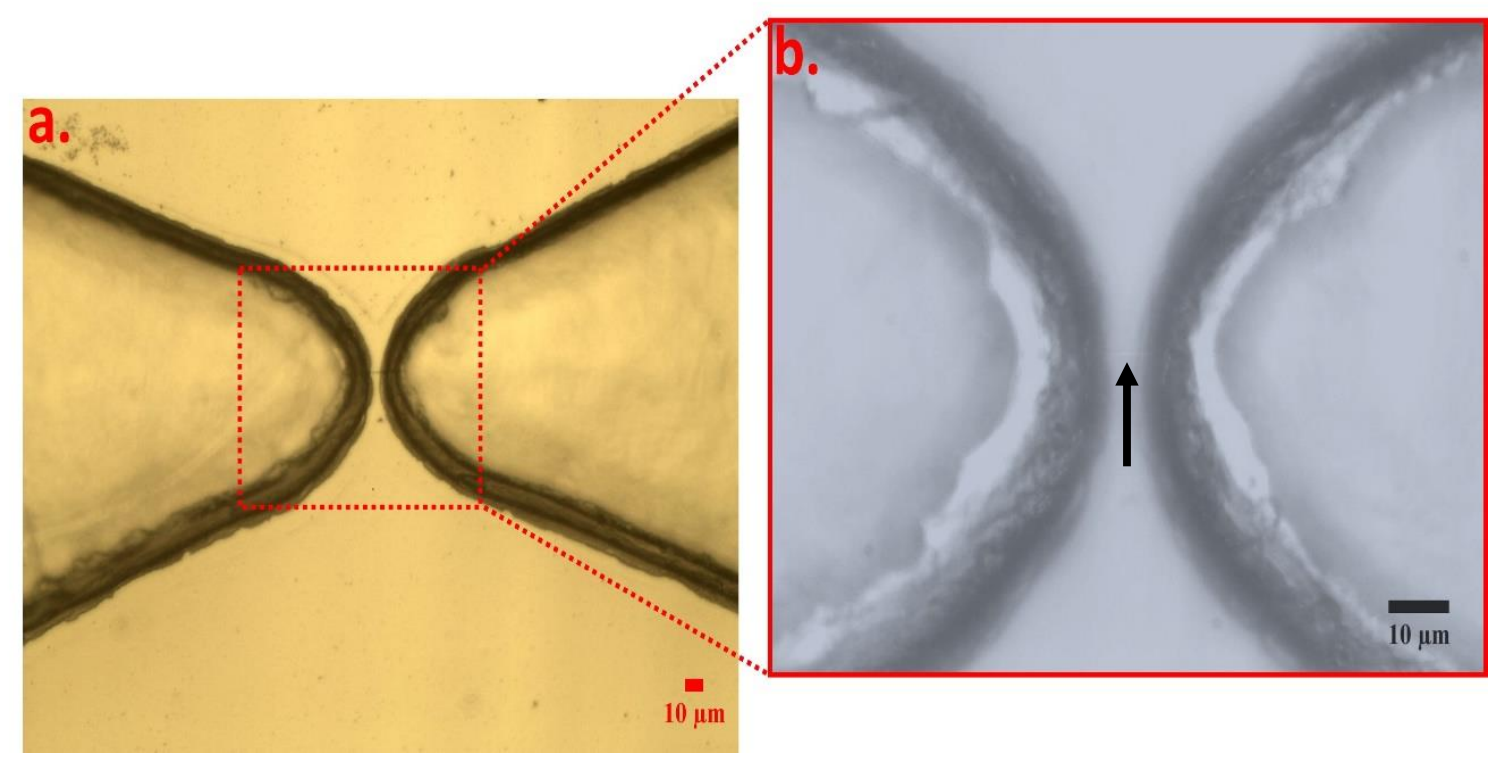

Figure 4. 23 a) Optical image of the Sub-Micron channel $(\sim 468 \mathrm{~nm})$ embedded within the PDMS "Dam", b) enlarged view of middle channel 


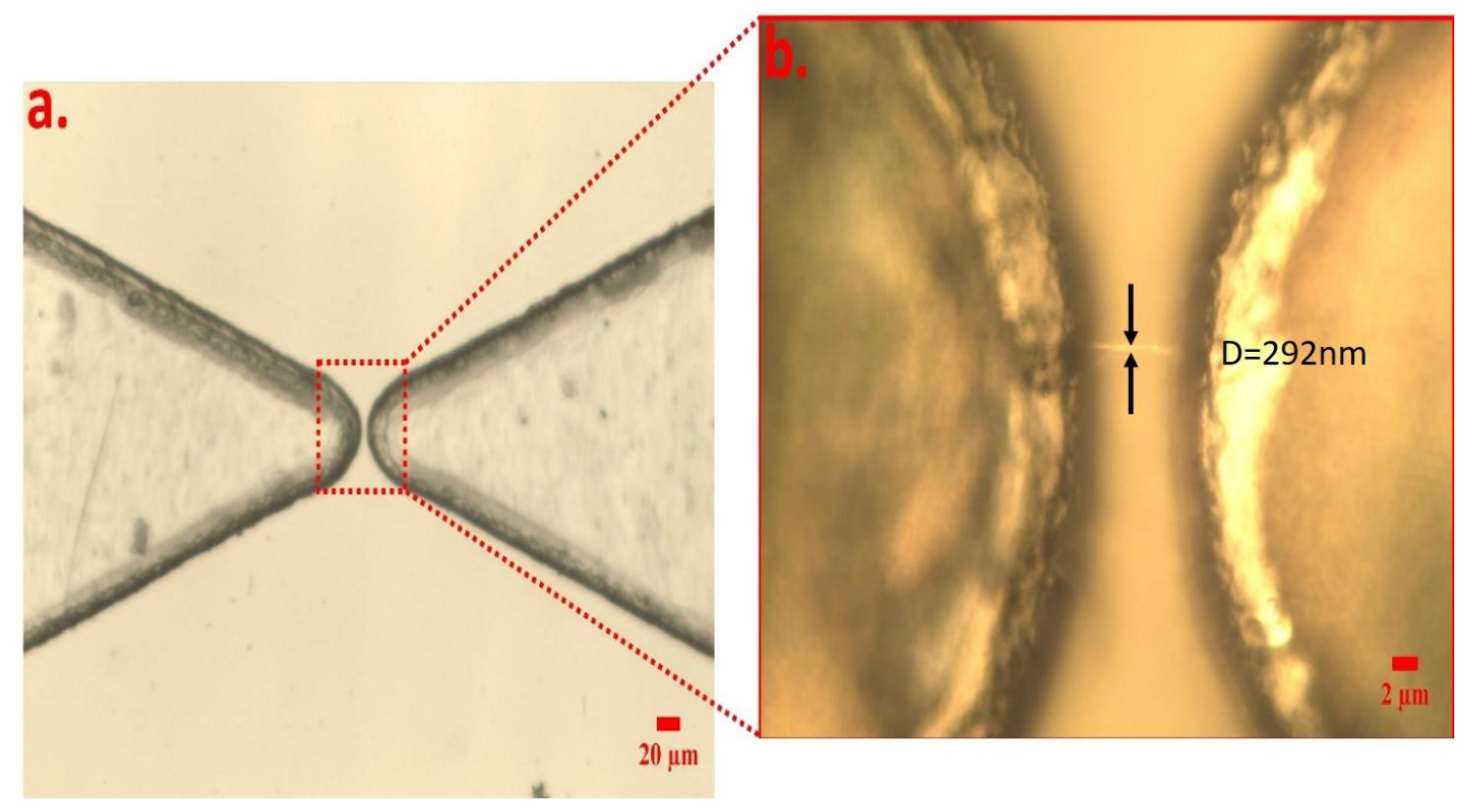

Figure 4. 24 a) Optical image of the Nanochannel ( 292 nm) embedded within the PDMS "Dam".

chapter, organic solvents were used to clean the surface of both substrates and to increase the adhesion before oxygen plasma. And oxygen plasma was treated on the both substrates for only one minute to increase the surface adhesion. Then, both substrates were immediately transferred to the $90{ }^{\circ} \mathrm{C}$ hotplate with light force applied on the top substrate.

\subsection{Transportation of Biomolecules}

Validation experiment were completed to prove the existence of the middle channel before further cellular electroporation experiments were performed. Here, PI dye was utilized to validate the middle micro/nanochannel and its connectivity to each side of the micro-chambers. Prior to performing these experiments, each microchamber was filled with PBS and the micro/nanochannel was allowed to fill. As previously explained, an external voltage $(6 \mathrm{~V})$ was applied across the channel with a negative potential applied to 
one microchamber and a positive potential applied to the other microchamber. Subsequently, the PI dye was loaded into the negative potential micro-chamber so the dye would travel through the middle channel into the positive potential micro-chamber (Figure 4.25). The migration of the PI dye across the micro/nanochannels was accomplished via electroosmotic and electrophoretic flow.

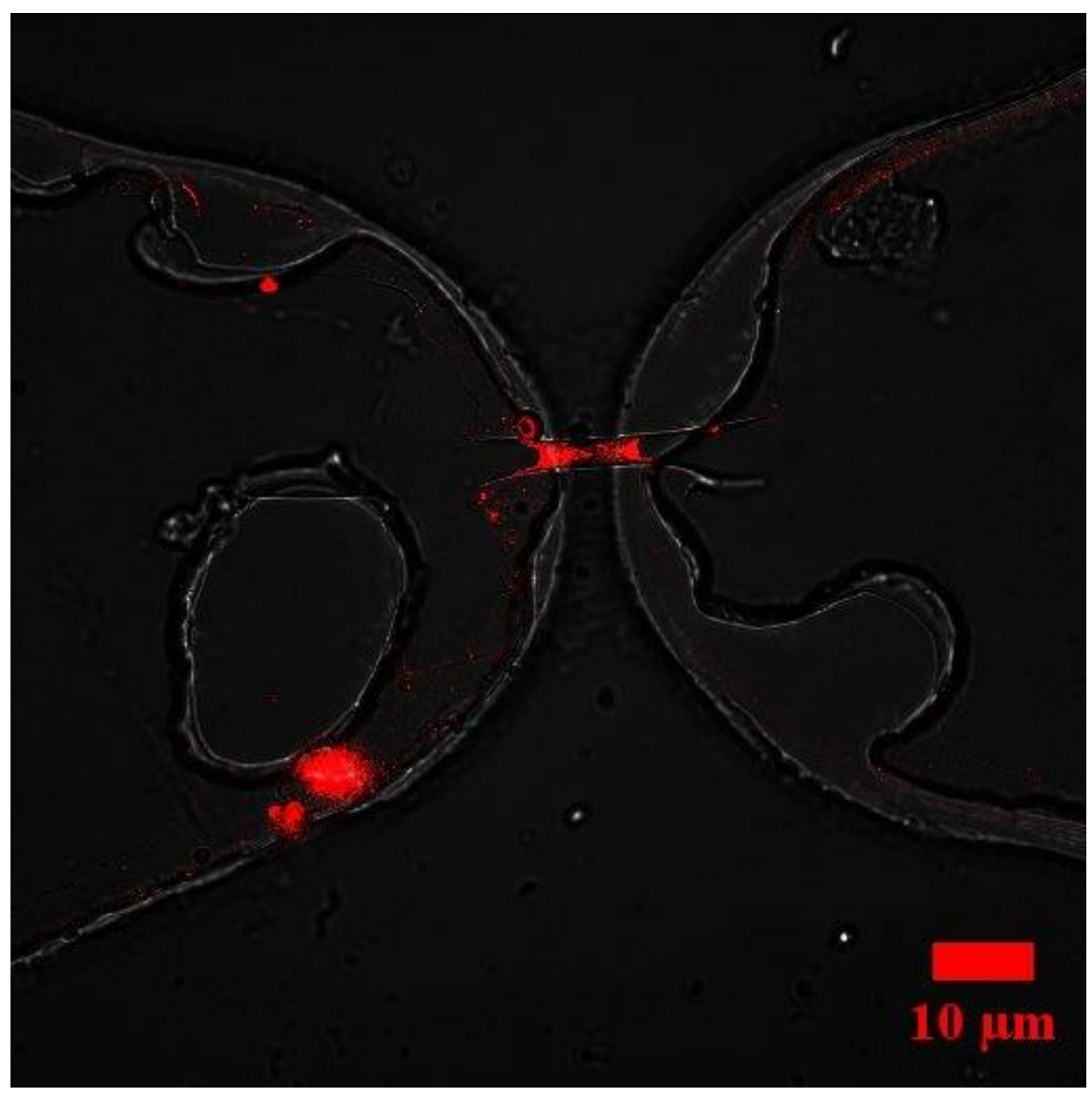

Figure 4. 25 Confocal microscope image of PI dye transporting through embedded microchannel $(3 \mu \mathrm{m})$. 


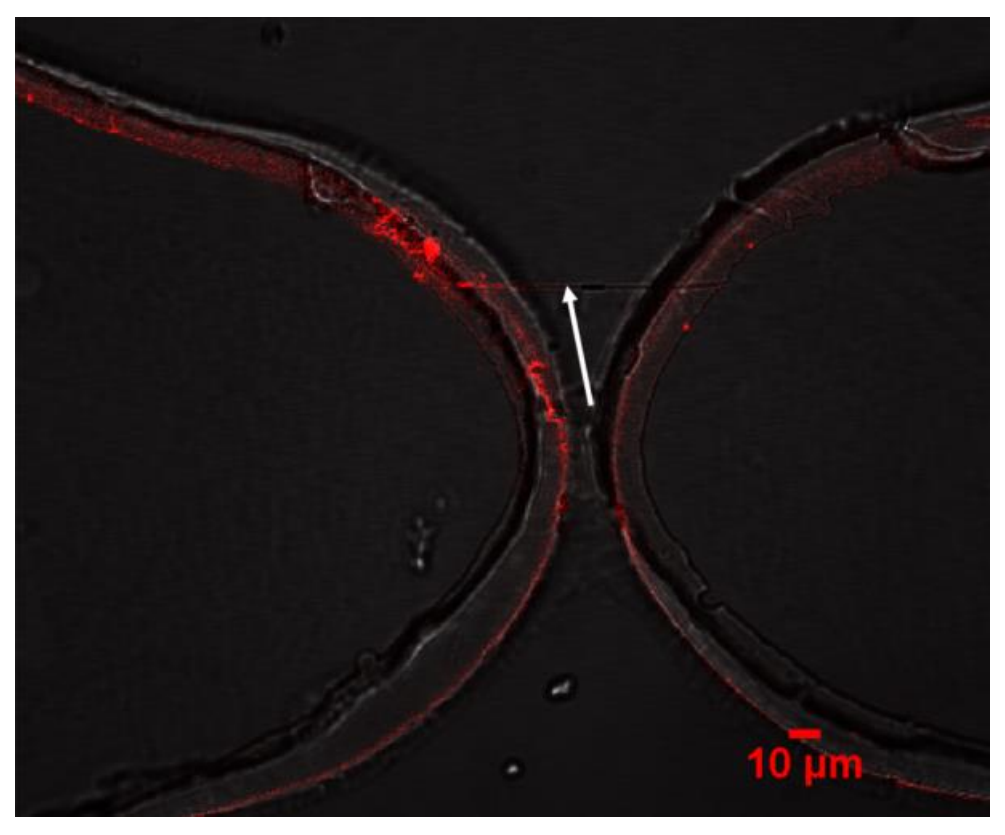

Figure 4. 26 Confocal microscope image of PI dye transporting through embedded micron channel (1112 nm).

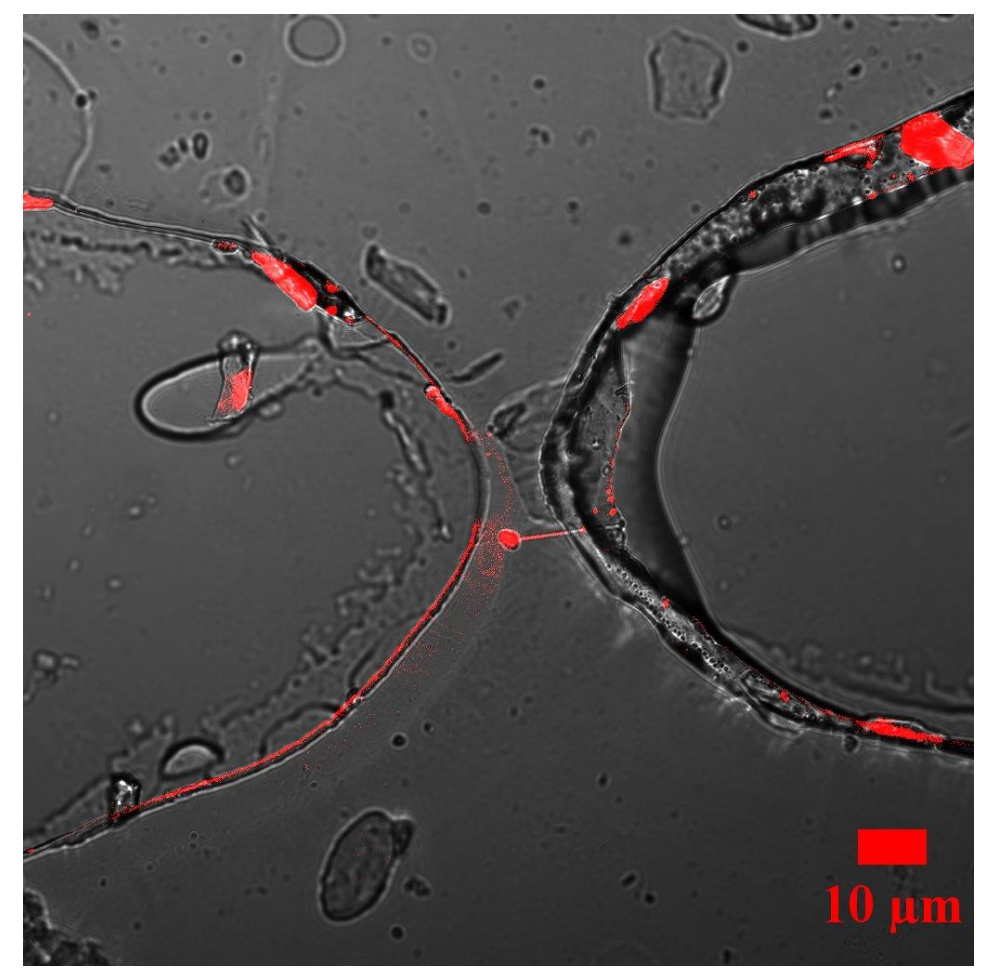

Figure 4. 27 Confocal microscope image of PI dye transporting through embedded Sub-micron channel $(518 \mathrm{~nm})$. 


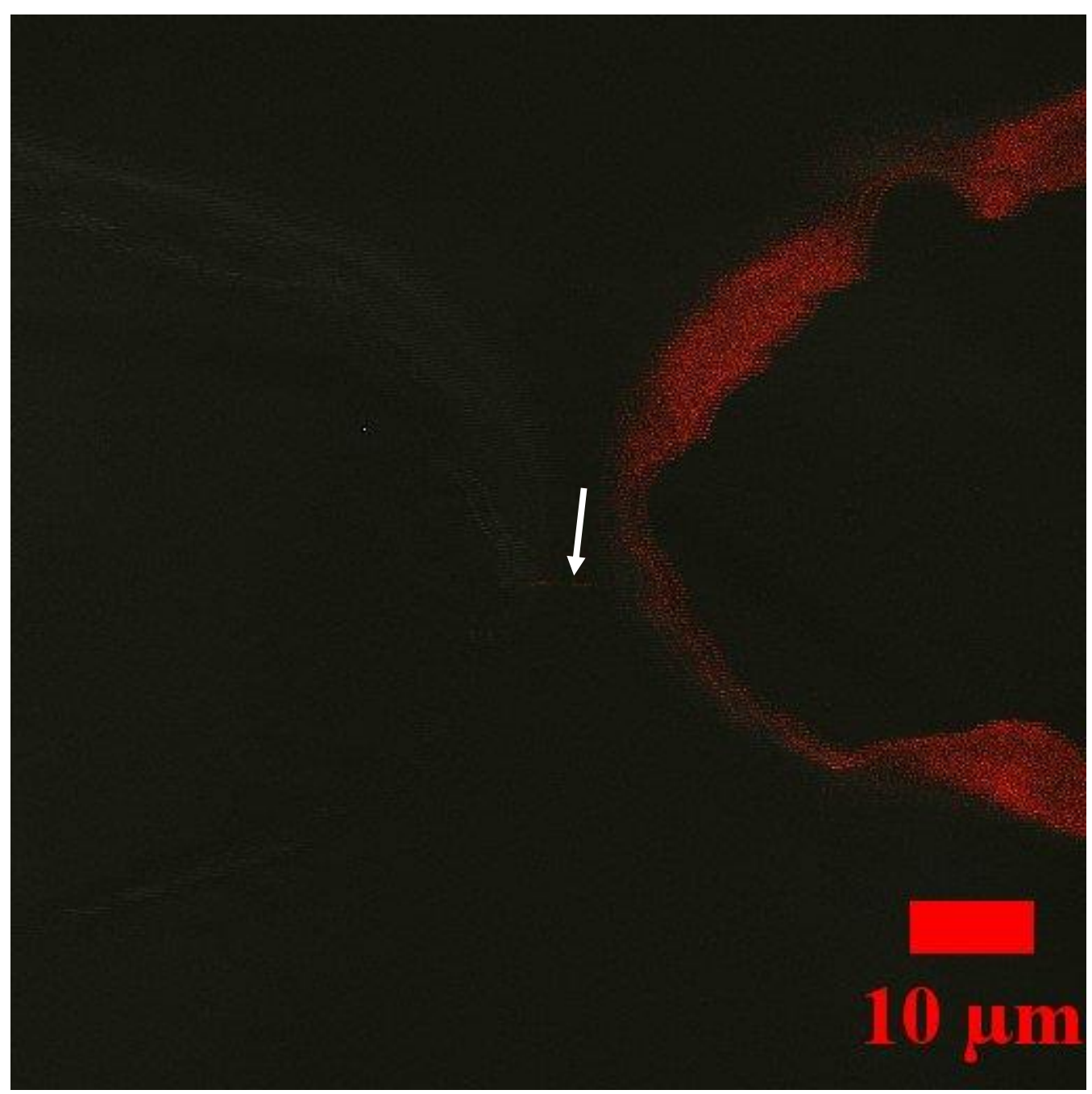

Figure 4. 28 Confocal microscope snapshot image of PI dye travelling through embedded nanochannel $(292 \mathrm{~nm})$.

Prior to performing the cellular transportation studies, each of the PDMS platforms, with the three different channel dimensions, were evaluated to validate that all channels were patent by viewing the nanochannel located in the middle "dam" under the confocal microscope. Both images and videos were recorded for each device with example for each channel dimension shown in Figures 4.26-4.28. 100\% of the 18 devices demonstrated flow through the micro/nanochannel. As a result, these devices were ready to perform the 
proposed cellular electroporation experiments. At the beginning, cells mortality was high when they were transferred to the nano/microfluidic devices and they were adhered to the bottom of the PDMS substrate. A standard sterilization process was executed before transferring any cells into the two chamber to avoid cell contamination and improve cell viability including autoclaving, baking, filtration, rinsing with ethanol and sterilized water, radiation with UV light. Coating a layer of BSA greatly reduced the adhesion of cells towards the bottom of PDMS substrate.

\subsection{Micro/Nano Electroporation}

\subsubsection{Optical Laser Trapping cells}

Prior to performing the electroporation studies, HL60 cells were loaded through the microchamber inlet port into the positive potential microchamber at a concentration of $1 \times 10^{7}$ cells $/ m L$. However, the cells were located upstream of the channel and needed to be moved to the outlet of the electroporation device. Due to the fluid resistance presented by the micro/nanochannels, the platforms were unable to move the cells via bulk fluid flow. Therefore, the cells were transported approximately $2.5 \mathrm{~mm}$ from the PDMS microchamber inlet port to the outlet of the electroporation device via optical laser trapping. By carefully controlling the laser intensity, cells, on at a time, were successfully and easily trapped and then translated via moving the stage of the microscope without damaging the cell (Figures 4.29 \& 4.30). More detailed time sequence images were also prepared (Figure 4.31) to illustrate the translation process of the stage to move the cell from its original location to the target area. The entire laser trapping process took approximately $12 s$ to complete. 


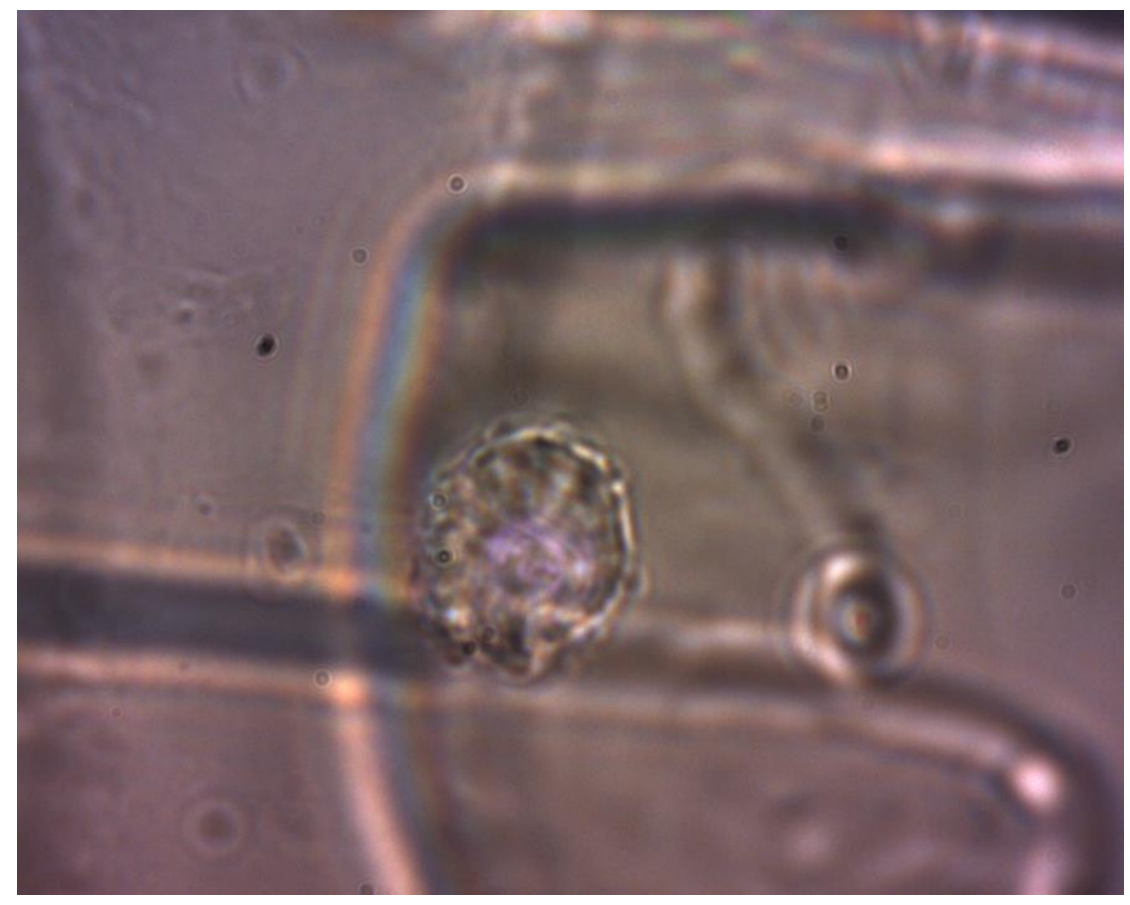

Figure 4. 29 Optical image of single cell trapped (wavelength is $980 \mathrm{~nm}$ ) at the entrance of the microchannel (dimension $3.27 \mu \mathrm{m}$ )

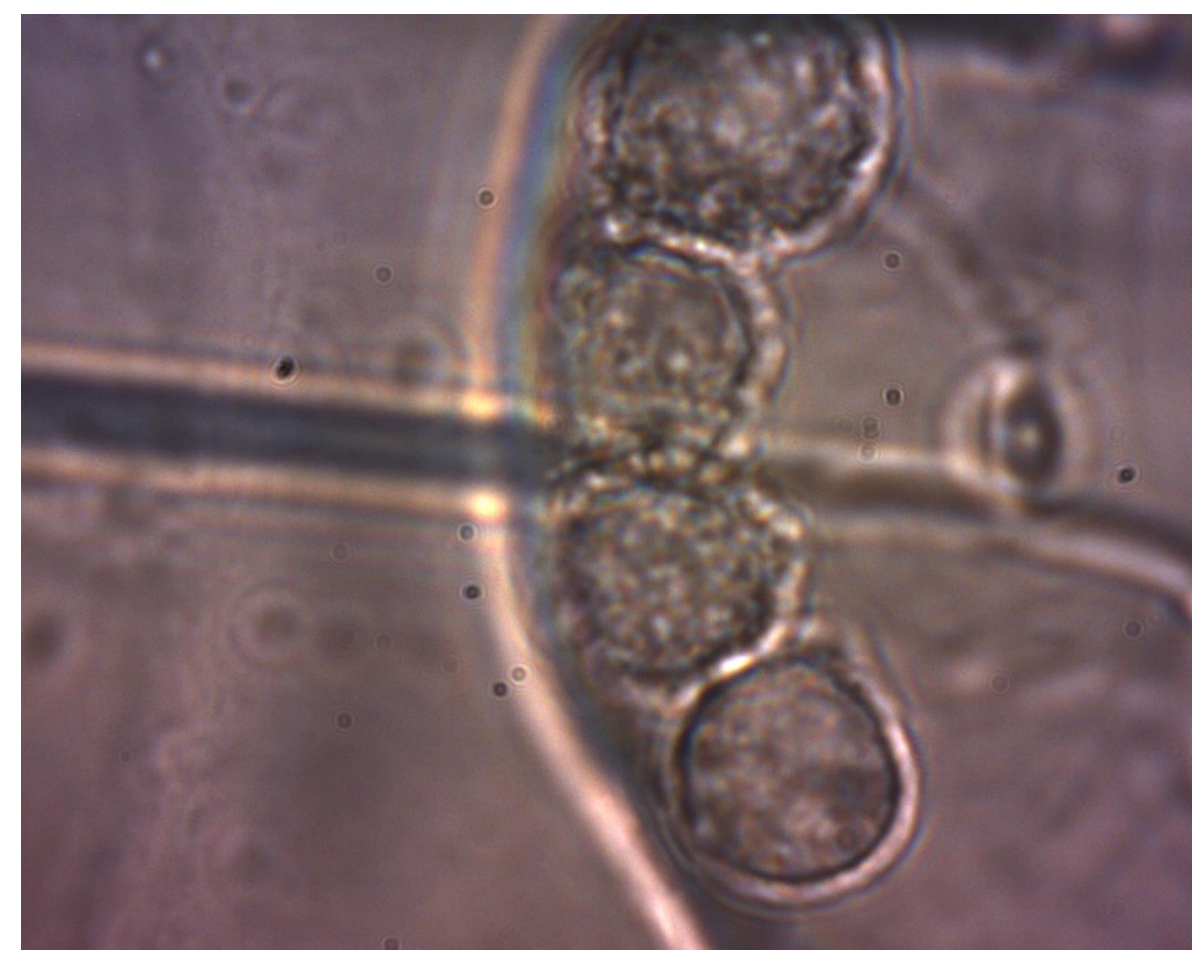

Figure 4. 30 Optical image of four cells laser trapped (wavelength is $980 \mathrm{~nm}$ ) at the entrance of the microchannel (dimension $3.12 \mu \mathrm{m}$ ) 

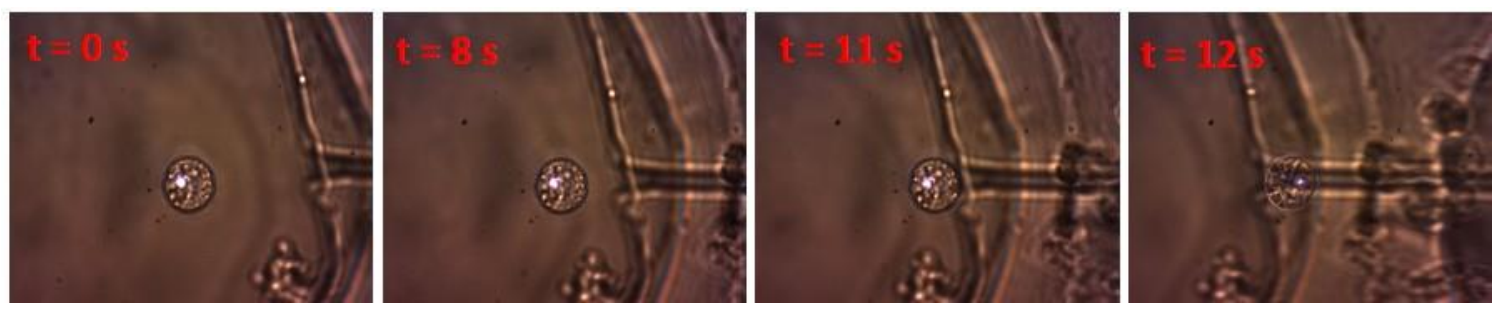

Figure 4. 31 Optical images of single cell trapping process (wavelength is $980 \mathrm{~nm}$ ).

\subsubsection{Micro/Nano Electroporation Preliminary Results}

Preliminary electroporation studies were performed to demonstrate proof-ofconcept and effectiveness of the platform to function as a nano-electroporation device. After the laser trapping procedure was completed, the platform was immediately transferred to the confocal microscope stage to perform the electroporation procedure. As depicted in the previous chapter, the power supply and two platinum electrodes were set in place prior to the electroporation procedure. Just prior to electroporation, an image of the micro/nanofluidic platform loaded with PI dye in micro-chamber and PBS together with cells in the other micro-chamber was taken to compare to the electroporation results (Figure 4.32). Subsequently, a $220 \mathrm{~V}$ with $10 \mathrm{~ms}$ pulse was applied on the two chambers to induce electroporation (here, a high voltage was used to generate high electric field to create a nanopore on the cell membrane; while only $6 \mathrm{~V}$ voltage provided enough force to migrate the PI dye ions from one chamber to the other one for biomolecules transportation). Within $20 \mathrm{~ms}$, this single trapped cell, positioned at the entrance of the nanochannel, was electroporated with the PI dye located in the other micro-chamber being transported through the nanochannel and transferred into the cell (Figures 4.32-4.35). Tens of electroporation experiments were performed with our platforms. The same amplitude and 


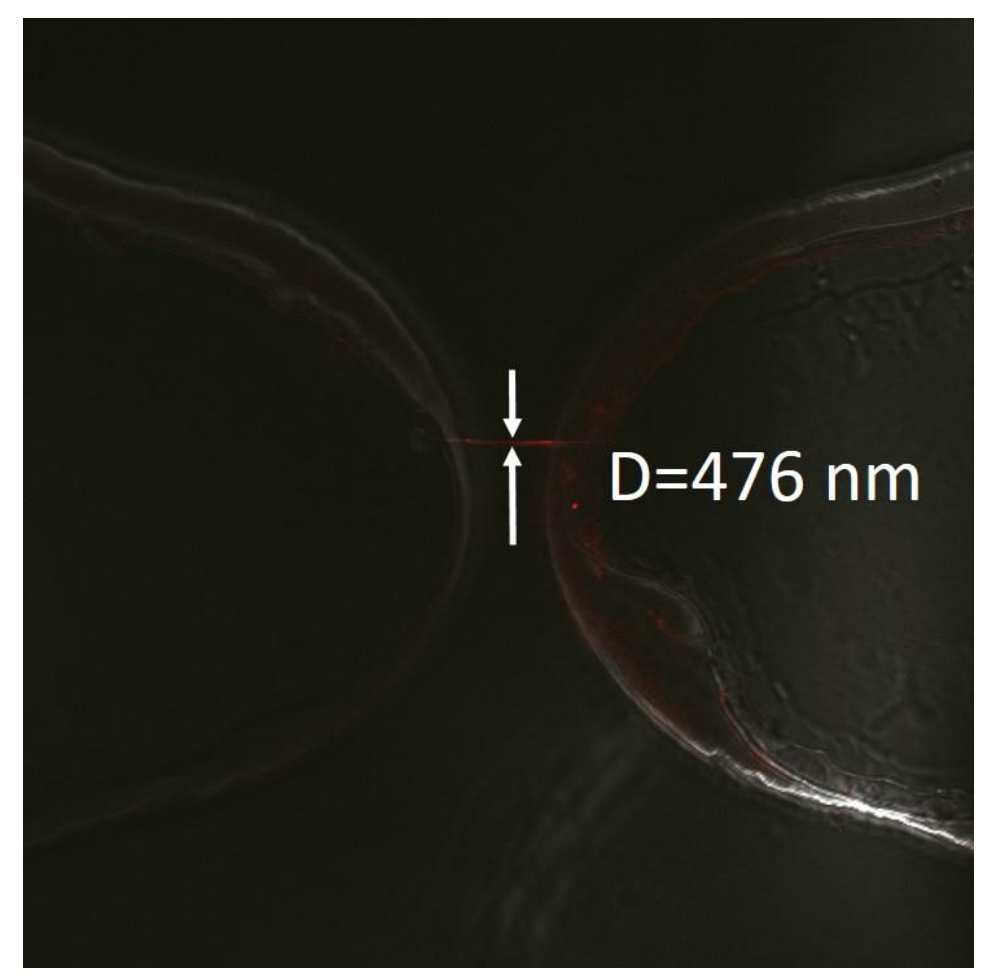

Figure 4. 32 Optical image of the platform before electroporation

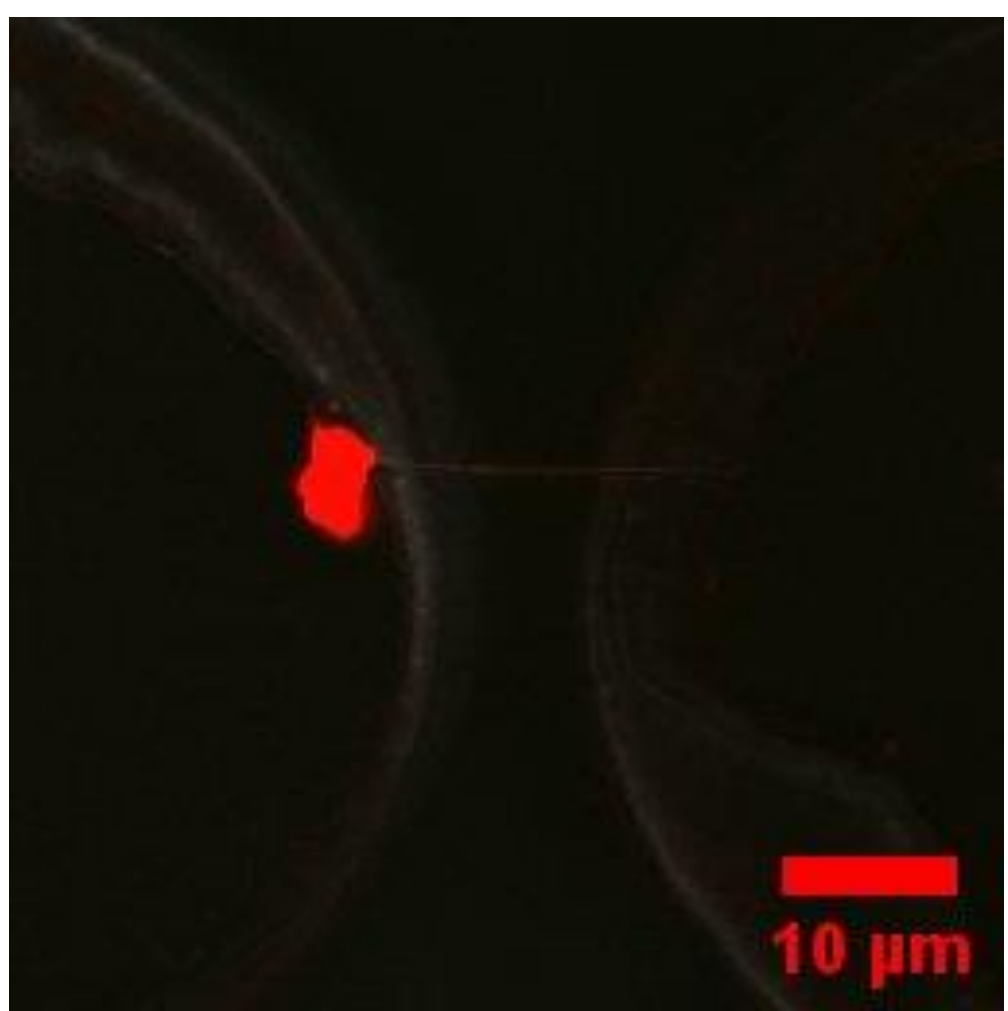

Figure 4. 33 Optical image of the platform (dimension $476 \mathrm{~nm}$ ) After Electroporation 


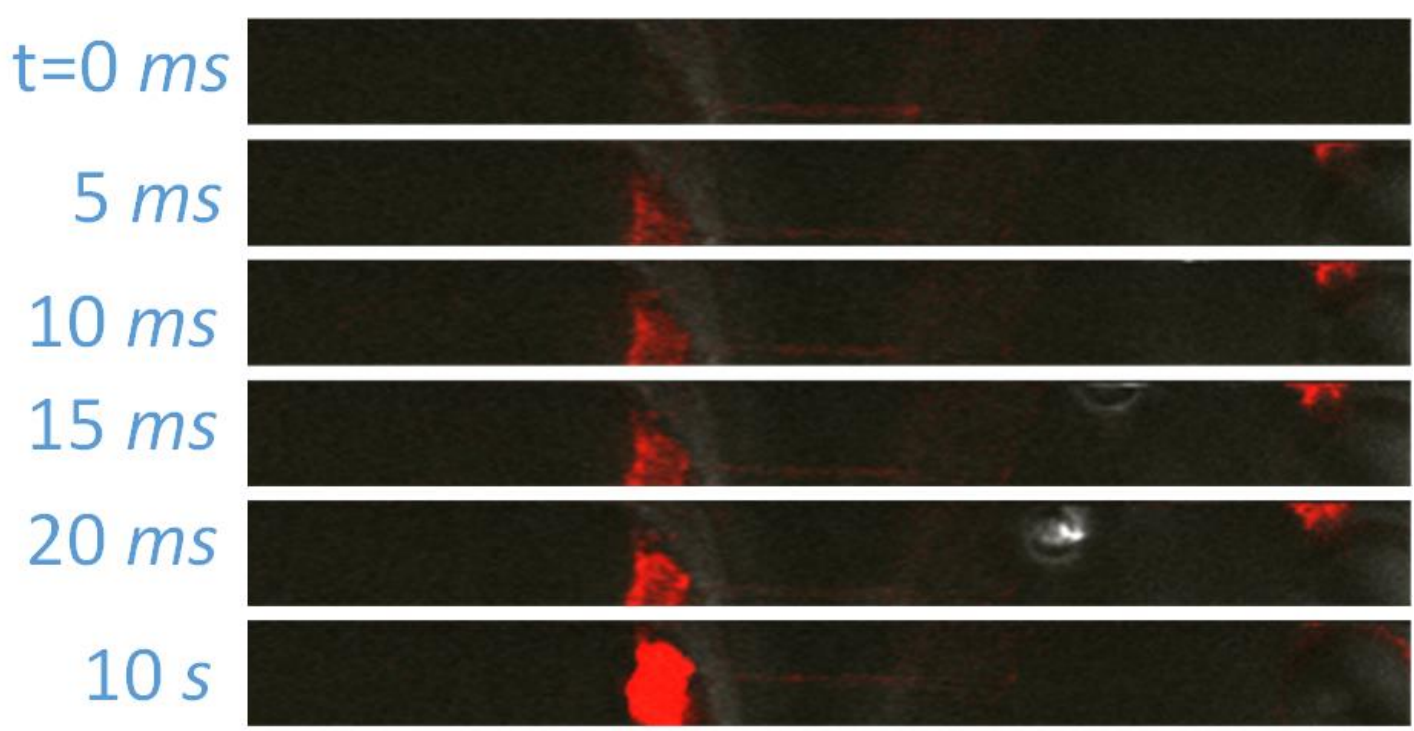

Figure 4. 34 Sequential images of single cell electroporation process for a $476 \mathrm{~nm}$ channel
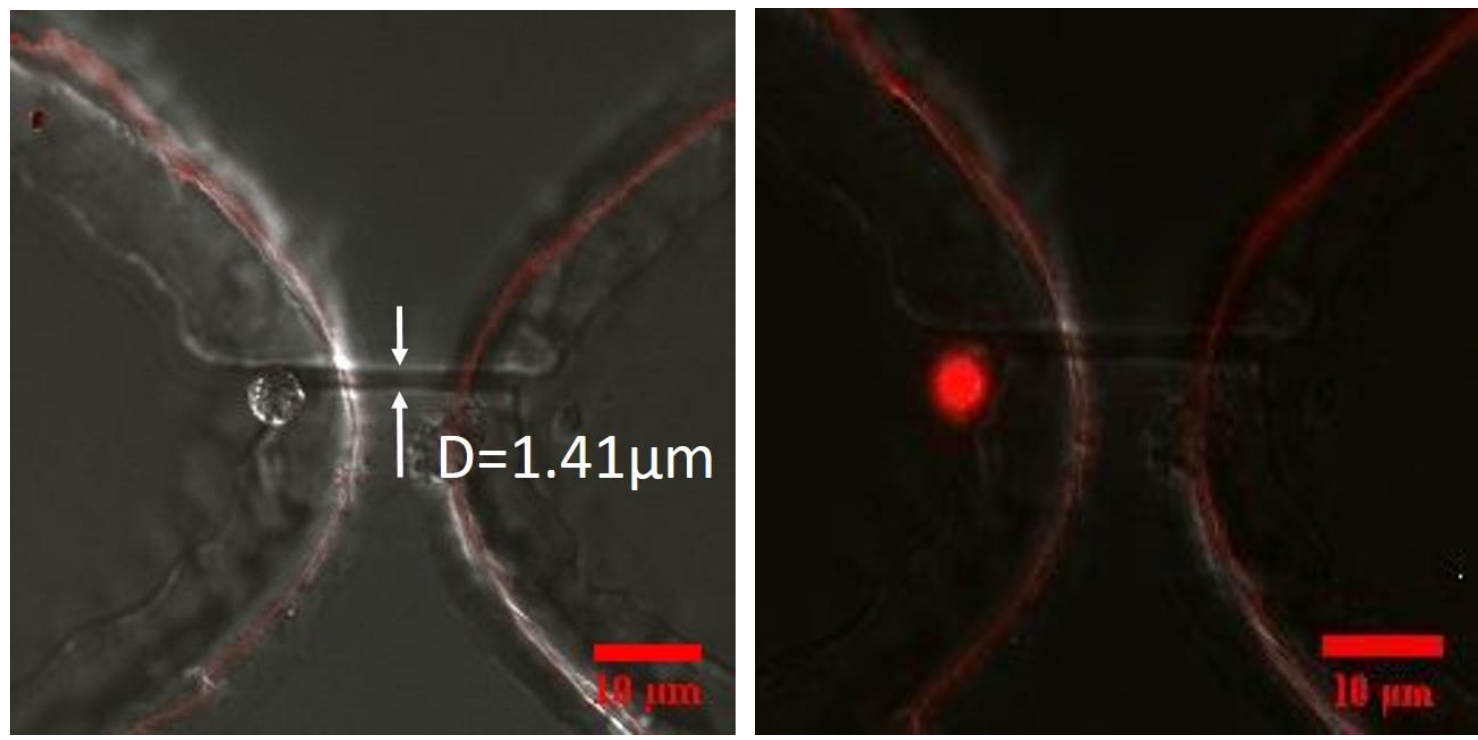

Figure 4. 35 Optical image of the platform (dimension 1.41) before and after electroporation

duration power was supplied to the device with the similar dimensions of successful platforms during the electroporation. High efficient cell transfection results were not observed in these devices because of some possible reasons: 1) cells were dead before 
performing the electroporation experiment; 2) middle channels were not completely connected to the two side's chambers. Much more careful sterilization process will need to be taken to avoid cell contamination in the future experiment. And some comparison experiments should be performed to determine whether cells are still alive or not before electroporation experiment. In addition, testing experiment on determining the connectivity of device to two side's microchambers should be performed including measuring resistance of middle channel filled with PBS solution and optical confirmation under microscope.

Compared to previous reported nano-electroporation results [36], the complete electroporation process in our platform only took around $20 \mathrm{~ms}$ to complete, which was 100 times shorter than the best reported result $(\sim 2.3 s)$ of Boukany et al. for a nanoscale electroporation device. As mentioned before, the micro/nanochannel in our studies laid at the middle level of the microchamber, which enabled the middle-level of the cell to lean against the entrance of the channel. On the one hand, the cell in our platform could have experienced a much larger electric field and transmembrane potential to create the nanopore, which accelerated the transport of the PI dye across the membrane. On the other hand, the biomolecules could have been more directly transported through the channel and transferred into the cell since the cell was in direct contact with the channel outlet. A 44 $\mathrm{V} / \mathrm{mm}$ electric field applied to our platform was much smaller than $110 \mathrm{~V} / \mathrm{mm}$ applied to Boukany's nanofluidic device. As discussed in the background chapter, the smaller electric field applied to the cell improved the cell viability as well as cell transfection efficiency. As the diameter of our platform's channel becomes smaller, the complete cell transfection 
time will become shorter and the dose of transferring biomolecules into cell can be more feasibly and precisely to control.

The conductivity of the PBS solution used for the electroporation was $2.1 \mathrm{~S} / \mathrm{m}$. The resistances of the micro/submicron/nanochannel were $1.5 \mathrm{M} \Omega, 6.1 \mathrm{M} \Omega$, and $16.8 \mathrm{M} \Omega$ respectively, which were calculated based on the following equation:

$$
R_{c h}=\left(\frac{A \sigma_{P B S}}{l}\right)^{-1}
$$

where $R_{c h}$ was the resistance of micro/submicron/nanochannel, $A$ was the cross section area of these channels, $\sigma_{P B S}$ was the conductivity of the PBS solution, and $l$ was the channel's length. The theoretical voltage applied on the cell was calculated based on the simulation model shown in Figure 2.1, where the total applied voltage was equal to the total voltage across channel and the cell membrane:

$$
V_{\text {total }}=V_{c h}+V_{T M 1}+V_{T M 2}
$$

where $V_{\text {total }}(220 \mathrm{~V}$ for this work's application) was the total voltage applied for the electroporation experiment, $V_{c h}$ was the voltage across the channel, $V_{T M 1}$ was the voltage across the half circle of the cell membrane close to the channel, and $V_{T M 2}$ was the voltage across the other half circle of the cell membrane. The equation for the transmembrane potential was derived from this simulation model and found to be:

$$
V_{T M 1}=V_{\text {total }} \frac{R_{1}}{R_{1}+R_{c h}}\left(1-e^{-t /\left(R_{c h} \cdot C_{1}\right)}\right)
$$

where $R_{1}$ was the resistance of the first half circle of the cell membrane, $C_{1}$ was the capacitance of the first half circle of the cell membrane (which was close to the channel), $t$ was the pulse period (10 $\mathrm{ms}$ for this work's application). A similar equation was used for the other half circle of the cell membrane to calculate $V_{T M 2}$. By combining the above 
equations and experimental parameters, the current flowing across the cell membrane (including first half circle and the other half circle) and energy passivation on the cell (Joule heat) were calculated (Table 4. 10). From this data, the current flow of the first half circle was found to be the same while the current flow for the second half circle was found to decrease as the channel dimension decreased. Similarly, the energy passivation was also found to decrease with smaller channel. These results indicated that smaller device produced much less energy, which means that the cell in the smaller device received less heat. To avoid damage towards to the cell in the large device including microfluidic device or bulk electroporation device, it was better to reduce the laser scanning time before electroporation experiment. The resistance $(1.6 M M \Omega)$ of the first circle was much larger that the resistance of middle channel and the second circle, which leads to the same current flow through it at different dimensions' devices.

Table 4. 9 Current flow and energy passivation across cell membrane for different device.

\begin{tabular}{|c|c|c|c|}
\hline $\begin{array}{c}\text { Design Dimension of } \\
\text { Channel }(\mathrm{nm})\end{array}$ & $\begin{array}{c}\text { Current Flow } 1^{\text {st }} \text { Circle } \\
(\mu \mathrm{A})\end{array}$ & $\begin{array}{c}\text { Current Flow 2 } \\
\text { Circle }(\mu \mathrm{A})\end{array}$ & $\begin{array}{c}\text { Energy Passivation } \\
(\mathrm{J})\end{array}$ \\
\hline 1000 & $1.38 \times 10^{-4}$ & 14.19 & $2.82 \times 10^{-3}$ \\
\hline 500 & $1.38 \times 10^{-4}$ & 10.95 & $1.67 \times 10^{-3}$ \\
\hline 300 & $1.38 \times 10^{-4}$ & 7.14 & $7.14 \times 10^{-4}$ \\
\hline
\end{tabular}




\section{CHAPTER 5 CONCLUSION}

\subsection{Work Completed}

A 3-axis robotic dispensing system was utilized to control the initiating and terminating positions of polymer fibers as well as fiber trajectory. The advantages of this robotic dispensing system were the system's ability to: 1) generate fibers with predetermined, desired diameters; 2) be programmed to dispense at precise locations; 3) be programmed for automated process control; and, 4) create complex micron/sub-micron fiber web structures. This new 3-axis robotic dispensing system generated significantly (P $=0.001$ ) better yields than previously reported systems using an ultra high-precision micromilling machine [48], 100\% verses 54.5\%, respectively, with the same polymer solution concentration, the fiber length range, and approximate feed rates. Therefore, hypothesis 1 has been proven based on the above comparisons between the two different systems.

Next, the study focused on characterizing the system to determine the key operating parameters of the system together with the material properties of the polymeric solutions to repeatedly create the desired sub-micron/nano scale PMMA fibers. An empirical prediction equation and optimization algorithm were developed and evaluated to verify their ability to accurately control fiber position, diameter and length. These models were utilized to determine the minimum theoretical fiber diameter the system was capable of producing and its corresponding experimental parameters. It was demonstrated that fibers fabricated from $1 \mu \mathrm{m}$ to $500 \mathrm{~nm}$, and $300 \mathrm{~nm}$ were within $20 \%$ of the design value (Table 
4. 10). Comparing the theoretical results with the experimental data showed that fibers fabricated at the minimum theoretical diameters (183 nm diameter) were repeatedly written with a $45 \%$ of the fibers having prediction error $\leq 15 \%$. Therefore, hypothesis 2 has been proven.

The PMMA fibers were successfully used as a sacrificial material, in conjunction with dry film photolithography, film-to-film alignment and bonding, and replica molding, to create micro and/or nano electroporation platforms. The fabrication techniques described herein present a new capability for creating micro/nano scale fluidic platforms with high accuracy. Compared to other traditional fabrication methods, the major advantages of this fabrication process included the ease of fabrication, low cost, and high potential for automation when using a nanofiber as a sacrificial structure for creating the nanochannel. In particular, contrasting our technique to soft photolithography, the method presented here simplified the manufacturing process by: 1) avoiding multilayer photolithography; 2) only needing two photomasks instead of multiple photomasks of different dimensions; 3) fabricating a mold that was used repeatedly; and, 4) producing fibers with a high yield $(\sim 95 \%)$. The method presented in this paper repeatedly created micron to nanoscale channels embedded in PDMS within 6\% of the channel design values, which has proven hypothesis 3 . Having the ability to readily and easily change the mold structures and micro/nano fibers' position and sizes aided in the production of customizable micro/nanofluidic platforms and directly addressed specific requirements and needs for particular biological applications.

The micro/nanofluidic electroporation platforms developed were used to successfully demonstrate their ability to perform biomolecule transport and nano 
electroporation in cells. First, the application of a low electric field $(44 \mathrm{~V} / \mathrm{mm})$ between the two micro-chambers induced PI dye transportion through the micro/nanochannel from one microchamber to the other for all 18 devices. The preliminary studies with HL60 cells indicated successful transportation (via electroporation) of the PI dye through the micro/nano-channel and into the cells. The total time for the entire electroporation process occurred within $20 \mathrm{~ms}$ with our platform compared to $2.3 \mathrm{~s}$ in Boukany et al.'s platform [36]; thus, hypothesis 4 has been proven.

\subsection{Future Plan}

In the future, more controlled electroporation experiments need to be implemented to prove the efficacy of these new designed electroporation platforms at microscale, submicroscale, and nanoscale. In addition, bulk electroporation of single cells also need to be performed in order to compare cell viability and electroporation times to the results obtained with the micro/sub-micro/nanoscale electroporation platforms. For future designs of this platform, some modifications may need to be made including increasing the height of two micro-chambers, replacing one access port with multiple access ports for the cell loading into the micro-chambers. Larger micro-chamber volume will lead to higher cell viability and success in cell transport during the laser trapping cell process. Multiple access

ports in the new design platforms would allow simultaneous electroporation of biomolecules into many single cells. 


\section{REFERENCES}

[1] S. Movahed and D. Li, "Microfluidics cell electroporation," Microfluidics and Nanofluidics, vol. 10, pp. 703-734, 2011.

[2] E. Neumann, M. Schaefer-Ridder, Y. Wang, and P. H. Hofschneider, "Gene transfer into mouse lyoma cells by electroporation in high electric fields," The EMBO journal, vol. 1, pp. 841-5, 1982.

[3] S. Movahed and D. Li, "A Theoretical Study of Single-Cell Electroporation in a Microchannel," The Journal of Membrane Biology, vol. 246, pp. 151-160, 2013.

[4] J. Li and H. Lin, "Numerical simulation of molecular uptake via electroporation," Bioelectrochemistry (Amsterdam, Netherlands), vol. 82, pp. 10-21, 2011.

[5] F. Aguel, K. A. Debruin, W. Krassowska, and N. A. Trayanova, "Effects of electroporation on the transmembrane potential distribution in a two-dimensional bidomain model of cardiac tissue," Journal of cardiovascular electrophysiology, vol. 10, pp. 701-14, 1999.

[6] S. Talele, P. Gaynor, M. J. Cree, and J. van Ekeran, "Modelling single cell electroporation with bipolar pulse parameters and dynamic pore radii," Journal of Electrostatics, vol. 68, pp. 261-274, 2010.

[7] X. Zhao, M. Zhang, and R. Yang, "Control of pore radius regulation for electroporation-based drug delivery," Communications in Nonlinear Science and Numerical Simulation, vol. 15, pp. 1400-1407, May 2010.

[8] S. Movahed and D. Li, "Electrokinetic transport through nanochannels," Electrophoresis, vol. 32, pp. 1259-67, 2011.

[9] D. A. Zaharoff, J. W. Henshaw, B. Mossop, and F. Yuan, "Mechanistic analysis of electroporation-induced cellular uptake of macromolecules," Experimental Biology and Medicine, vol. 233, pp. 94-105, Jan 2008.

[10] Y. Granot, B. Rubinsky, and I. Biomedical-Related Special, "Mass transfer model for drug delivery in tissue cells with reversible electroporation," International Journal of Heat and Mass Transfer, vol. 51, pp. 5610-5616, 2008.

[11] S. Movahed and D. Li, "Electrokinetic transport through the nanopores in cell membrane during electroporation," Journal of Colloid And Interface Science, vol. 369, pp. 442-452, 2012.

[12] J. Weaver and Y. Chizmadzhev, "Theory of electroporation: A review," Bioelectrochemistry and Bioenergetics, vol. 41, pp. 135-160, 1996.

[13] M. S. Venslauskas and S. Satkauskas, "Mechanisms of transfer of bioactive molecules through the cell membrane by electroporation," European Biophysics Journal, 2015.

[14] J. C. Weaver, "Electroporation of cells and tissues," IEEE Transactions on Plasma Science, vol. 28, pp. 24-33, 2000. 
[15] Y. Lee and P. Deng, "Review of micro/nano technologies and theories for electroporation of biological cells," Science China Physics, Mechanics and Astronomy, vol. 55, pp. 996-1003, 2012.

[16] T. Santra and F. Tseng, "Recent Trends on Micro/Nanofluidic Single Cell Electroporation," Micromachines, vol. 4, pp. 333-356, 2013.

[17] S. Wang and L. J. Lee, "Micro-/nanofluidics based cell electroporation," Biomicrofluidics, vol. 7, p. 11301, 2013.

[18] R. C. Lee, L. P. River, F. S. Pan, L. Ji, and R. L. Wollmann, "Surfactant-induced sealing of electropermeabilized skeletal muscle membranes in vivo," Proceedings of the National Academy of Sciences, vol. 89, pp. 4524-4528, 1992.

[19] M. P. Rols, C. Delteil, G. Serin, and J. Teissie, "Temperature effects on electrotransfection of mammalian cells," NUCLEIC ACIDS RESEARCH, vol. 22, p. 540, 1994.

[20] J. W. Loomis-Husselbee, P. J. Cullen, R. F. Irvine, and A. P. Dawson, "Electroporation can cause artefacts due to solubilization of cations from the electrode plates. Aluminum ions enhance conversion of inositol 1,3,4,5tetrakisphosphate into inositol 1,4,5-trisphosphate in electroporated L1210 cells," ed, 1991.

[21] Available: Steven-Weber-chemistry.squarespace.com

[22] M. Wang, O. Orwar, J. Olofsson, and S. G. Weber, "Single-cell electroporation," Analytical and Bioanalytical Chemistry, vol. 397, pp. 3235-3248, 2010.

[23] H. Yuan, S. D. Cambron, M. M. Crain, and R. S. Keynton, "Fabrication of a Micro/Nanofluidic Platform Via Three-Axis Robotic Dispensing System," Journal of Micro and Nano-Manufacturing, vol. 4, pp. 041005-041005-6, 2016.

[24] U. Zimmermann, J. Schulz, and G. Pilwat, "Transcellular ion flow in Escherichia coli B and electrical sizing of bacterias," Biophysical journal, vol. 13, pp. 1005-13, 1973.

[25] H. G. Coster and U. Zimmermann, "Dielectric breakdown in the membranes of Valonia utricularis. The role of energy dissipation," Biochimica et biophysica acta, vol. 382, pp. 410-8, 1975.

[26] U. Zimmermann, F. Riemann, and G. Pilwat, "Enzyme loading of electrically homogeneous human red blood cell ghosts prepared by dielectric breakdown," $B B A$ - Biomembranes, vol. 436, pp. 460-474, 1976.

[27] K. Kinosita, Jr. and T. Y. Tsong, "Formation and resealing of pores of controlled sizes in human erythrocyte membrane," Nature, vol. 268, pp. 438-41, 1977.

[28] K. Kinosita, Jr. and T. Y. Tsong, "Survival of sucrose-loaded erythrocytes in the circulation," Nature, vol. 272, pp. 258-60, 1978.

[29] R. Benz, F. Beckers, and U. Zimmermann, "Reversible electrical breakdown of lipid bilayer membranes: A charge-pulse relaxation study," The Journal of Membrane Biology, vol. 48, pp. 181-204, 1979.

[30] R. Benz and U. Zimmermann, "Relaxation studies on cell membranes and lipid bilayers in the high electric field range," Journal of Electroanalytical Chemistry and Interfacial Electrochemistry, vol. 116, pp. 723-739, 1980/01/01 1980.

[31] J. Teissie and T. Y. Tsong, "Electric field induced transient pores in phospholipid bilayer vesicles," Biochemistry, vol. 20, pp. 1548-54, 1981. 
[32] R. C. Lee, L. P. River, F. S. Pan, L. Ji, and R. L. Wollmann, "Surfactant-induced sealing of electropermeabilized skeletal muscle membranes in vivo," Proceedings of the National Academy of Sciences of the United States of America, vol. 89, pp. 4524-8, 1992.

[33] M. P. Rols, C. Delteil, G. Serin, and J. Teissié, "Temperature effects on electrotransfection of mammalian cells," Nucleic acids research, vol. 22, p. 540, 1994.

[34] J. W. Loomis-Husselbee, P. J. Cullen, R. F. Irvine, and A. P. Dawson, "Electroporation can cause artefacts due to solubilization of cations from the electrode plates. Aluminum ions enhance conversion of inositol 1,3,4,5tetrakisphosphate into inositol 1,4,5-trisphosphate in electroporated L1210 cells," Biochemical Journal, vol. 277, pp. 883-885, 1991.

[35] J. A. Lundqvist, F. Sahlin, M. A. I. Åberg, A. Strömberg, P. S. Eriksson, and O. Orwar, "Altering the biochemical state of individual cultured cells and organelles with ultramicroelectrodes," Proceedings of the National Academy of Sciences, vol. 95, pp. 10356-10360, September 1, 19981998.

[36] P. E. Boukany, A. Morss, W.-c. Liao, B. Henslee, H. Jung, X. Zhang, et al., "Nanochannel electroporation delivers precise amounts of biomolecules into living cells," Nature Nanotechnology, vol. 6, pp. 747-754, 2011.

[37] J. Rathenberg, T. Nevian, and V. Witzemann, "High-efficiency transfection of individual neurons using modified electrophysiology techniques," Journal of Neuroscience Methods, vol. 126, pp. 91-98, 6/15/ 2003.

[38] M. Khine, A. Lau, C. Ionescu-Zanetti, J. Seo, and L. P. Lee, "A single cell electroporation chip," Lab on a Chip, vol. 5, pp. 38-43, 2005.

[39] Y. Huang and B. Rubinsky, "Flow-through micro-electroporation chip for high efficiency single-cell genetic manipulation," Sensors \& Actuators A: Physical, vol. 104, 2003.

[40] Y.-C. Lin, C.-M. Jen, M.-Y. Huang, C.-Y. Wu, and X.-Z. Lin, "Electroporation microchips for continuous gene transfection," Sensors and Actuators B: Chemical, vol. 79, pp. 137-143, 10/15/ 2001.

[41] J. Seo, C. Ionescu-Zanetti, J. Diamond, R. Lal, and L. P. Lee, "Integrated multiple patch-clamp array chip via lateral cell trapping junctions," Applied Physics Letters, vol. 84, 2004.

[42] Y.-C. Lin, M. Li, C.-S. Fan, and L.-W. Wu, "A microchip for electroporation of primary endothelial cells," Sensors and Actuators A: Physical, vol. 108, pp. 12-19, 11/15/ 2003.

[43] Y.-C. Lin, M. Li, and C.-C. Wu, "Simulation and experimental demonstration of the electric field assisted electroporation microchip for in vitro gene delivery enhancement," Lab on a Chip, vol. 4, pp. 104-108, 2004.

[44] M. Khine, C. Ionescu-Zanetti, A. Blatz, L.-P. Wang, and L. P. Lee, "Single-cell electroporation arrays with real-time monitoring and feedback control," Lab on a Chip, vol. 7, pp. 457-462, 2007.

[45] Z. Fei, S. Wang, Y. Xie, B. E. Henslee, C. G. Koh, and L. J. Lee, "Gene transfection of mammalian cells using membrane sandwich electroporation," Analytical chemistry, vol. 79, pp. 5719-22, 2007. 
[46] S.-C. Chen, T. S. Santra, C.-J. Chang, T.-J. Chen, P.-C. Wang, and F.-G. Tseng, "Delivery of molecules into cells using localized single cell electroporation on ITO micro-electrode based transparent chip," Biomedical Microdevices : BioMEMS and Biomedical Nanotechnology, vol. 14, pp. 811-817, 2012.

[47] M. Fox, D. Esveld, A. Valero, R. Luttge, H. Mastwijk, P. Bartels, et al., "Electroporation of cells in microfluidic devices: a review," Analytical and Bioanalytical Chemistry, vol. 385, pp. 474-485, 2006.

[48] S. M. Berry, "Characterization of a direct-write method for fabricating 3D polymer microfibers and construction of microscale platforms," 2008.

[49] Double Layer. Available: https://en.wikipedia.org/wiki/Double layer_interfacial)

[50] M. Smoluchowski and A. Krakauer, vol. 182, 1903.

[51] D. Halliday, R. Resick, and J. Walker, Fundamentals of physics. 7th ed. Hoboken, NJ: Wiley, 2005.

[52] F. M. White, Fluid mechanics, 4th ed. ed. Boston, Mass. :: WCB/McGraw-Hill, 1999.

[53] A. Jouyban-Gharamaleki, M. G. Khaledi, and B. J. Clark, "Calculation of electrophoretic mobilities in water-organic modifier mixtures in capillary electrophoresis," Journal of Chromatography A, vol. 868, pp. 277-284, 2000.

[54] H. Becker, A. Manz, and H. Becker, Microsystem technology in chemistry and life science. Berlin ;: Springer, 1998.

[55] B. Ziaie, A. Baldi, M. Lei, Y. Gu, and R. A. Siegel, "Hard and soft micromachining for BioMEMS: review of techniques and examples of applications in microfluidics and drug delivery," Advanced Drug Delivery Reviews, vol. 56, pp. 145-172, 2/10/ 2004.

[56] M. Albin, "Book Review:Microsystem Technology: A Powerful Tool for Biomolecular Studies J. M. Kohler, T. Mejevaia, H. P. Saluz," The Quarterly Review of Biology, vol. 75, 2000.

[57] A. C. R. Grayson, R. S. Shawgo, A. M. Johnson, N. T. Flynn, Y. Li, M. J. Cima, et al., "A BioMEMS Review: MEMS Technology for Physiologically Integrated Devices (Invited Paper)," PROCEEDINGS-IEEE, vol. 92, pp. 6-21, 2004.

[58] J. West, M. Becker, S. Tombrink, and A. Manz, "Micro Total Analysis Systems: Latest Achievements," Analytical Chemistry, vol. 80, 2008.

[59] R. F. Service, "Can sensors make a home in the body?," Science (New York, N.Y.), vol. 297, pp. 962-3, 2002.

[60] D. J. Wirthlin, F. Alcocer, D. Whitley, and W. D. Jordan, "Use of hybrid aortic stent grafts for endovascular repair of abdominal aortic aneurysms: indications and outcomes," J Surg Res, vol. 108, pp. 14-9, Nov 2002.

[61] J. T. Santini, Jr., M. J. Cima, and R. Langer, "A controlled-release microchip," Nature, vol. 397, pp. 335-8, 1999.

[62] J. Jing, E. H. T. Francis, M. Jianmin, and I. Ciprian, "Microfabricated microneedle with porous tip for drug delivery," Journal of Micromechanics and Microengineering, vol. 16, pp. 958-964, 2006.

[63] P. R. Troyk, "Injectable Electronic Identification, Monitoring, and Stimulation Systems," Annual Review of Biomedical Engineering, vol. 1, pp. 177-209, 1999. 
[64] G. S. Fiorini and D. T. Chiu, "Disposable microfluidic devices: fabrication, function, and application," Biotechniques, vol. 38, pp. 429-446, Mar 2005.

[65] M. J. Mescher, E. E. L. Swan, J. Fiering, M. E. Holmboe, W. F. Sewell, S. G. Kujawa, et al., "Fabrication Methods and Performance of Low-Permeability Microfluidic Components for a Miniaturized Wearable Drug Delivery System," Journal of Microelectromechanical Systems, vol. 18, pp. 501-510, Jun 2009.

[66] J. Cheng, C.-s. Liu, S. Shang, D. Liu, W. Perrie, G. Dearden, et al., "A review of ultrafast laser materials micromachining," Optics \& Laser Technology, vol. 46, pp. 88-102, 3// 2013.

[67] P. Cardoso and J. P. Davim, "A BRIEF REVIEW ON MICROMACHINING OF MATERIALS," Reviews on Advanced Materials Science, vol. 30, pp. 98-102, Feb 2012.

[68] A. TermehYousefi, S. Bagheri, and N. Adib, "Integration of biosensors based on microfluidic: a review," Sensor Review, vol. 35, pp. 190-199, 2015.

[69] S. Filiz, C. M. Conley, M. B. Wasserman, and O. B. Ozdoganlar, "An experimental investigation of micro-machinability of copper 101 using tungsten carbide microendmills," International Journal of Machine Tools and Manufacture, vol. 47, pp. 1088-1100, 6// 2007.

[70] K. Ren, J. Zhou, and H. Wu, "Materials for Microfluidic Chip Fabrication," Accounts of Chemical Research, vol. 46, pp. 2396-2406, 2013/11/19 2013.

[71] L. Zema, G. Loreti, A. Melocchi, A. Maroni, and A. Gazzaniga, "Injection Molding and its application to drug delivery," Journal of Controlled Release, vol. 159, pp. 324-331, 5/10/ 2012.

[72] P. Kim, K. W. Kwon, M. C. Park, S. H. Lee, S. M. Kim, and K. Y. Suh, "Soft Lithography for Microfluidics: a Review," BIOCHIP JOURNAL, vol. 2, pp. 1-11, March 2008.

[73] H. Yuan, S. Cambron, M. Crain, and R. Keynton, "Fabrication of a Micron/Nanofluidic Platform via 3-Axis Robotic Dispensing System," p. V001T02A085, 2016.

[74] D. Qin, Y. Xia, and G. M. Whitesides, "Soft lithography for micro- and nanoscale patterning," Nat. Protocols, vol. 5, pp. 491-502, 03//print 2010.

[75] A. P. Quist, E. Pavlovic, and S. Oscarsson, "Recent advances in microcontact printing," Analytical and Bioanalytical Chemistry, vol. 381, pp. 591-600, 2005.

[76] S. Alom Ruiz and C. S. Chen, "Microcontact printing: A tool to pattern," Soft Matter, vol. 3, pp. 168-177, 2007.

[77] K. Y. Suh, Y. S. Kim, and H. H. Lee, "Capillary Force Lithography," Advanced Materials, vol. 13, pp. 1386-1389, 2001.

[78] K. Y. Suh and H. H. Lee, "Capillary Force Lithography: Large-Area Patterning, Self-Organization, and Anisotropic Dewetting," Advanced Functional Materials, vol. 12, pp. 405-413, 2002.

[79] S. M. Cho, K. Young Jun, K. Yong Shin, Y. Yoonseok, and H. Seung-Chul, "The application of carbon nanotube-polymer composite as gas sensing materials," in Sensors, 2004. Proceedings of IEEE, 2004, pp. 701-704 vol.2.

[80] A. K. Ekaputra, G. D. Prestwich, S. M. Cool, and D. W. Hutmacher, "Combining Electrospun Scaffolds with Electrosprayed Hydrogels Leads to Three-Dimensional 
Cellularization of Hybrid Constructs," Biomacromolecules, vol. 9, pp. 2097-2103, 2008.

[81] F. Xu, P. Horak, and G. Brambilla, "Optical microfiber coil resonator refractometric sensor," Optics express, vol. 15, pp. 7888-93, 2007.

[82] P. D. Dalton, N. T. Joergensen, J. Groll, and M. Moeller, "Patterned melt electrospun substrates for tissue engineering," Biomedical materials (Bristol, England), vol. 3, p. 034109, 2008.

[83] A. Hadjizadeh, C. J. Doillon, and P. Vermette, "Bioactive polymer fibers to direct endothelial cell growth in a three-dimensional environment," Biomacromolecules, vol. 8, pp. 864-73, 2007.

[84] C. Xu, "Aligned biodegradable nanofibrous structure: a potential scaffold for blood vessel engineering," Biomaterials, vol. 25, pp. 877-886, 2004.

[85] Y. You, B. M. Min, S. J. Lee, T. S. Lee, and W. H. Park, "In Vitro Degradation Behavior of Electrospun Polyglycolide, Polylactide, and Poly(lactide-coglycolide)," JOURNAL OF APPLIED POLYMER SCIENCE, vol. 95, pp. 193-200, 2005.

[86] S. M. Berry, S. P. Warren, D. A. Hilgart, A. T. Schworer, S. Pabba, A. S. Gobin, et al., "Endothelial cell scaffolds generated by 3D direct writing of biodegradable polymer microfibers," Biomaterials, vol. 32, pp. 1872-9, 2011.

[87] A. B. Dalton, S. Collins, E. Munoz, J. M. Razal, V. H. Ebron, J. P. Ferraris, et al., "Super-tough carbon-nanotube fibres," Nature, vol. 423, 2003.

[88] A. B. Dalton, S. Collins, J. Razal, E. Munoz, V. H. Ebron, B. G. Kim, et al., "Continuous carbon nanotube composite fibers: properties, potential applications, and problems," Journal of Materials Chemistry, vol. 14, pp. 1-3, 2003.

[89] L. H. Sperling, Introduction to physical polymer science, 2nd ed. ed. New York :: Wiley, 1992.

[90] S. A. Harfenist, S. D. Cambron, E. W. Nelson, S. M. Berry, A. W. Isham, M. M. Crain, et al., "Direct Drawing of Suspended Filamentary Micro- and Nanostructures from Liquid Polymers," Nano Letters, vol. 4, 2004.

[91] G. Wu, H. Li, Y. Wu, and J. A. Cuculo, "Structure and property studies of poly(trimethylene terephthalate) high-speed melt spun fibers," Polymer, vol. 43, pp. 4915-4922, 8// 2002.

[92] J. M. Samon, J. M. Schultz, and B. S. Hsiao, "Structure development in the early stages of crystallization during melt spinning," Polymer, vol. 43, pp. 1873-1875, 2002.

[93] J. K. W. Sandler, S. Pegel, M. Cadek, F. Gojny, M. van Es, J. Lohmar, et al., "A comparative study of melt spun polyamide-12 fibres reinforced with carbon nanotubes and nanofibres," Polymer, vol. 45, pp. 2001-2015, 3// 2004.

[94] G. Wu, Q. Li, and J. A. Cuculo, "Fiber structure and properties of poly(ethylene2,6-naphthalate) obtained by high-speed melt spinning," Polymer, vol. 41, pp. $8139-8150,10 / / 2000$.

[95] J. Zeng, B. Saltysiak, W. S. Johnson, D. A. Schiraldi, and S. Kumar, "Processing and properties of poly(methyl methacrylate)/carbon nano fiber composites," Composites Part B: Engineering, vol. 35, pp. 173-178, 3// 2004. 
[96] J. D. Schiffman and C. L. Schauer, "A Review: Electrospinning of Biopolymer Nanofibers and their Applications," Polymer Reviews, vol. 48, pp. 317-352, 2008/05/01 2008.

[97] D. Li, J. T. McCann, Y. Xia, and M. Marquez, "Electrospinning: A Simple and Versatile Technique for Producing Ceramic Nanofibers and Nanotubes," Journal of the American Ceramic Society, vol. 89, pp. 1861-1869, 2006.

[98] Li, D, Wang, Y, and Xia, "Electrospinning of Polymeric and Ceramic Nanofibers as Uniaxially Aligned Arrays," Nano Letters, vol. 3, 2003.

[99] A. Theron, E. Zussman, and A. L. Yarin, "Electrostatic field-assisted alignment of electrospun nanofibres," Nanotechnology, vol. 12, pp. 384-390, 2001.

[100] W. E. Teo and S. Ramakrishna, "A review on electrospinning design and nanofibre assemblies," Nanotechnology, vol. 17, pp. R89-R106, 2006.

[101] R. Yang, J. He, L. Xu, and J. Yu, "Bubble-electrospinning for fabricating nanofibers," Polymer, vol. 50, pp. 5846-5850, 2009.

[102] S. Kaur, S. Sundarrajan, D. Rana, R. Sridhar, R. Gopal, T. Matsuura, et al., "Review: the characterization of electrospun nanofibrous liquid filtration membranes," Journal of Materials Science : Full Set - Includes 'Journal of Materials Science Letters', vol. 49, pp. 6143-6159, 2014.

[103] S. Pabba, "Generalization and extensions of capillary thinning driven self-assembly of nanostructured air-bridges," 2009.

[104] G. H. McKinley, "VISCO-ELASTO-CAPILLARY THINNING AND BREAK-UP OF COMPLEX FLUIDS," RHEOLOGY REVIEWS, pp. 1-48, 2005.

[105] R. B. Bird and R. B. Bird, Dynamics of polymeric liquids, 2nd ed. ed. New York :: Wiley, 1987.

[106] R. B. Bird, "INVITED REVIEW Polymer Kinetic Theories and Elongational Flowsdagger," Chemical Engineering Communications, vol. 16, pp. 175-187, 1982/08/01 1982.

[107] D. F. James, "Presheared Extensional Flow of Dilute Polymer Solutions," Journal of Rheology, vol. 31, p. 453, 1987.

[108] S. M. Berry, S. Pabba, J. L. Fernades, J. M. Rathfon, G. N Tew, A. S. Gobin, et al., "Direct fabrication by manual brushing of suspended microscale fibers for cell culture scaffolds," in Society for Biomaterials Annual Conference, Chicago, IL., 2007.

[109] Surface tension. Available: https://en.wikipedia.org/wiki/Surface tension

[110] H. Yuan, S. D. Cambron, and R. S. Keynton, "Prescribed 3-D Direct Writing of Suspended Micron/Sub-micron Scale Fiber Structures via a Robotic Dispensing System," Journal of Visualized Experiments, p. 52834, 2015.

[111] Viscosity. Available: https://en.wikipedia.org/wiki/Viscosity

[112] Available: http://www.ias.ac.in/initiat/sci ed/resources/chemistry/Viscosity.pdf

[113] A. Safir, M.M. Yazdanpanah, S. Pabba, S. D. Cambron, F. P. Zamborini, R. S. Keynton, et al., "An insulated nanowire probe for electrochemical sensing in cells," presented at the Annual KY EPSCoR Conference, Louisville, KY, 15 MAY 2006.

[114] S. Pabba, A. SAfir, M. M. Yazdanpanah, and R. W. Cohn, "Direct self assembly of nanostructures," presented at the E-EXPO, University of Louisville, Louisville, KY, 4 March 2006. 
[115] A. Tripathi, P. Whittingstall, and G. H. McKinley, "Using filament stretching rheometry to predict strand formation and "processability" in adhesives and other non-Newtonian fluids," Rheologica Acta : An International Journal of Rheology, vol. 39, pp. 321-337, 2000.

[116] M. I. Kolte and P. Szabo, "Capillary thinning of polymeric filaments," Journal of Rheology, vol. 43, pp. 609-625, 1999.

[117] G. H. McKinley and A. Tripathi, "How to extract the Newtonian viscosity from capillary breakup measurements in a filament rheometer," Journal of Rheology, vol. 44, pp. 653-670, 2000.

[118] G. Vozzi, C. Flaim, A. Ahluwalia, and S. Bhatia, "Fabrication of PLGA scaffolds using soft lithography and microsyringe deposition," Biomaterials, vol. 24, pp. 2533-2540, 6// 2003.

[119] S. M. Berry, S. Pabba, J. Crest, S. D. Cambron, G. H. McKinley, R. W. Cohn, et al., "Characterization and modeling of direct-write fabrication of microscale polymer fibers," Polymer, vol. 52, pp. 1654-1661, 2011.

[120] M. B. Scott, A. H. Steven, W. C. Robert, and S. K. Robert, "Characterization of micromanipulator-controlled dry spinning of micro- and sub-microscale polymer fibers," Journal of Micromechanics and Microengineering, vol. 16, p. 1825, 2006.

[121] JR-C Points Software. Available: http://regmed.musc.edu/atbc/files/PPManuals/PC\%20Operation.pdf

[122] M. A. Mohammed and R. Holder, "Introducing analysis of means to medical statistics," BMJ Quality \& Safety, April 17, 20122012.

[123] S. M. Berry, S. Pabba, R. W. Cohn, and R. S. Keynton, "Direct-Write Drawing of Carbon Nanotube/Polymer Composite Microfibers," Journal of Nanomaterials, vol. 2012, pp. 1-8, 2012.

[124] W. M. v. Spengen, "Capacitive RF MEMS switch dielectric charging and reliability: a critical review with recommendations," Journal of Micromechanics and Microengineering, vol. 22, p. 074001, 2012.

[125] V. Paul, H. Till, A. Björn, and G. A. Urban, "A full-wafer fabrication process for glass microfluidic chips with integrated electroplated electrodes by direct bonding of dry film resist," Journal of Micromechanics and Microengineering, vol. 19, 2009.

[126] K. Stephan, P. Pittet, L. Renaud, P. Kleimann, P. Morin, N. Ouaini, et al., "Fast prototyping using a dry film photoresist: microfabrication of soft-lithography masters for microfluidic structures," Journal of Micromechanics and Microengineering, vol. 17, pp. N69-N74, 2007.

[127] P. Vulto, N. Glade, L. Altomare, J. Bablet, L. D. Tin, G. Medoro, et al., "Microfluidic channel fabrication in dry film resist for production and prototyping of hybrid chips," Lab on a Chip, vol. 5, pp. 158-162, 2005.

[128] J. Nilsson, M. Evander, B. Hammarström, and T. Laurell, "Review of cell and particle trapping in microfluidic systems," Analytica Chimica Acta, vol. 649, pp. $141-157,9 / 7 / 2009$.

[129] C. Piggee, "Optical tweezers: not just for physicists anymore," Analytical Chemistry, vol. 81, pp. 16-19, 2009/01/01 2009.

[130] Reed Relay. Available: http://www.aleph-eu.com/product/p/SIP-SD

[131] US-6009. Available: http://www.ni.com/pdf/manuals/371303n.pdf 
[132] H. W. Robert, "Confocal optical microscopy," Reports on Progress in Physics, vol. 59, p. 427, 1996.

[133] Nikon. Available: http://www2.egr.uh.edu/ nvaradar/private/Inst/Nikon.pdf

[134] N. R. Draper, H. Smith, and H. E. Smith, Applied regression analysis, 2d ed. ed. New York :: Wiley, 1981.

[135] A. J. Chipperfield and P. J. Fleming, "The MATLAB genetic algorithm toolbox," in Applied Control Techniques Using MATLAB, IEE Colloquium on, 1995, pp. 10/1-10/4.

Film Lamination.

Available:

http://louisville.edu/micronano/files/documents/standard-operatingprocedures/DryFilmLaminationSOP.pdf 


\section{APPENDIX A}

\section{Dry Film Lamination SOP [136]}

\section{Procedure:}

1. Turn on the power of Dry Film Laminator.

2. Hit the "Preheat" button to ensure the red "preheat" is on after set the preheating temperature at around $230^{\circ} \mathrm{F}$ and running speed at 5 .

3. Wait about 5 mins to let the Dry Film Laminator reach to the presetting temperature at around $230{ }^{\circ} \mathrm{F}$.

4. Place the substrate at the entry tray of the laminator which is coated with one end of the dry film resists (one protection layer towards the substrate was removed before).

5. Hit the "Run" button and lift up the other end of the dry film resists during running process.

6. Use a pair of scissors or a razor blade to trim dry film resists away from the substrate.

7. Turn off the Dry Film Laminator. 


\section{APPENDIX B}

\section{Coil ratings of the reed relay [130]}

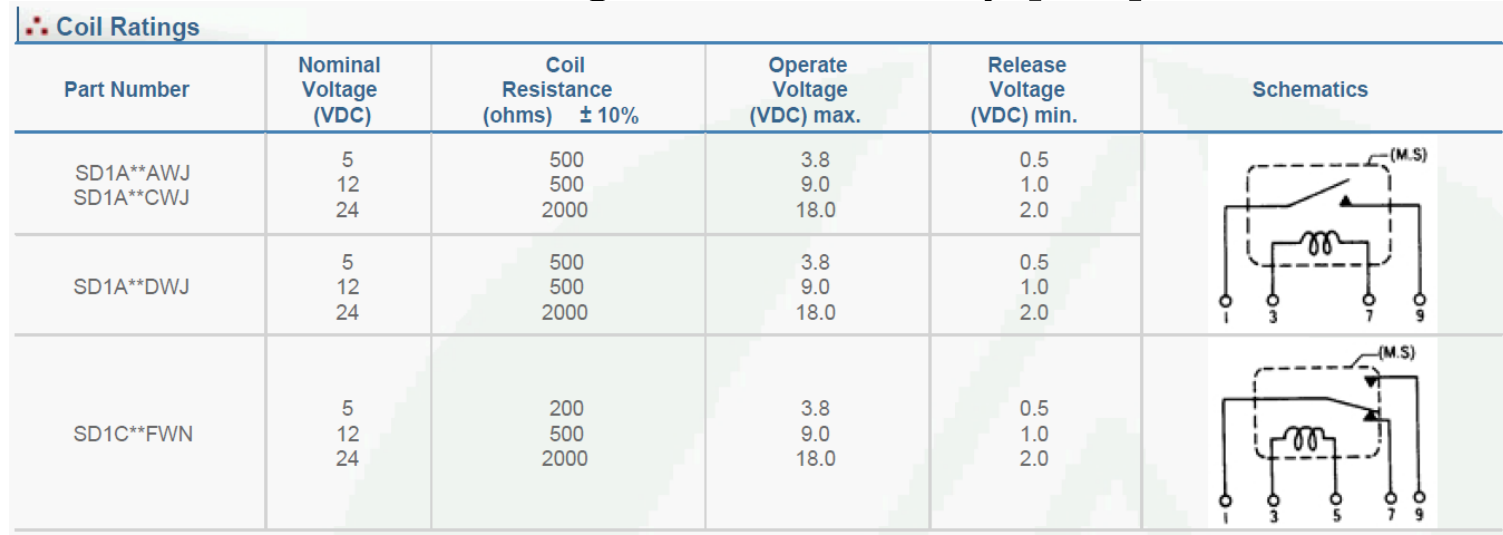

Contact ratings of the reed relay [130]

$\therefore$ Contact Ratings

\begin{tabular}{|c|c|c|c|c|c|}
\hline \multicolumn{2}{|l|}{ Parameters } & SD1A*AWJ & SD1A**CWJ & SD1A**DW & SD1C**FWN \\
\hline \multicolumn{2}{|l|}{ Power (W) max. } & 10 & 50 & 25 & 3 \\
\hline \multicolumn{2}{|l|}{ Voltage (VDC) max. } & 200 & 200 & 1000 & 100 \\
\hline \multicolumn{2}{|l|}{ Switching Current (A) max. } & 0.5 & 1.0 & 1.0 & 0.25 \\
\hline \multicolumn{2}{|c|}{ Carry Current (A) max. } & 1.0 & 2.0 & 2.0 & 0.5 \\
\hline \multicolumn{2}{|c|}{ Contact Resistance (ohms) max. (initial) } & 0.2 & 0.2 & 0.2 & 0.2 \\
\hline \multirow{2}{*}{$\begin{array}{l}\text { Breakdown Voltage (VDC) } \\
\text { min. }\end{array}$} & $\begin{array}{l}\text { Open } \\
\text { Contact }\end{array}$ & 250 & 300 & 1500 & 200 \\
\hline & $\begin{array}{l}\text { Contact to } \\
\text { Coil }\end{array}$ & 1500 & 1500 & 1500 & 1500 \\
\hline \multirow{2}{*}{$\begin{array}{l}\text { Insulation Resistance (ohms) } \\
\text { min. }\end{array}$} & $\begin{array}{l}\text { Open } \\
\text { Contact }\end{array}$ & $10^{10}$ & $10^{10}$ & $10^{10}$ & $10^{8}$ \\
\hline & $\begin{array}{l}\text { Contact to } \\
\text { Coil }\end{array}$ & $10^{10}$ & $10^{10}$ & $10^{10}$ & $10^{10}$ \\
\hline \multicolumn{2}{|c|}{ Operate Time (mS) $\max$ (incl. bounce) } & 1.0 & 1.0 & 1.0 & 2.0 \\
\hline \multicolumn{2}{|c|}{ Release Time (mS) max. } & 0.5 & 0.5 & 0.5 & 2.5 \\
\hline \multirow{2}{*}{ Electrical Life } & Low Level & $\begin{array}{c}10^{8}(10 \mathrm{mVDC} \\
10 \mu \mathrm{A})\end{array}$ & & & $\begin{array}{c}5 \times 10^{6}(10 \mathrm{VDC}, \\
4 \mathrm{~mA})\end{array}$ \\
\hline & Rated Load & $\begin{array}{c}6 \times 10^{5}(20 \mathrm{VDC}, \\
0.5 \mathrm{~A})\end{array}$ & $\begin{array}{c}5 \times 10^{5}(200 \mathrm{VDC}, \\
0.25 \mathrm{~A})\end{array}$ & $\begin{array}{c}2 \times 10^{6}(1000 \mathrm{VDC}, \\
10 \mathrm{~mA})\end{array}$ & $\begin{array}{c}2 \times 10^{6}(12 \mathrm{VDC}, \\
0.25 \mathrm{~A})\end{array}$ \\
\hline
\end{tabular}


Dimensional drawing of the reed relay [130]

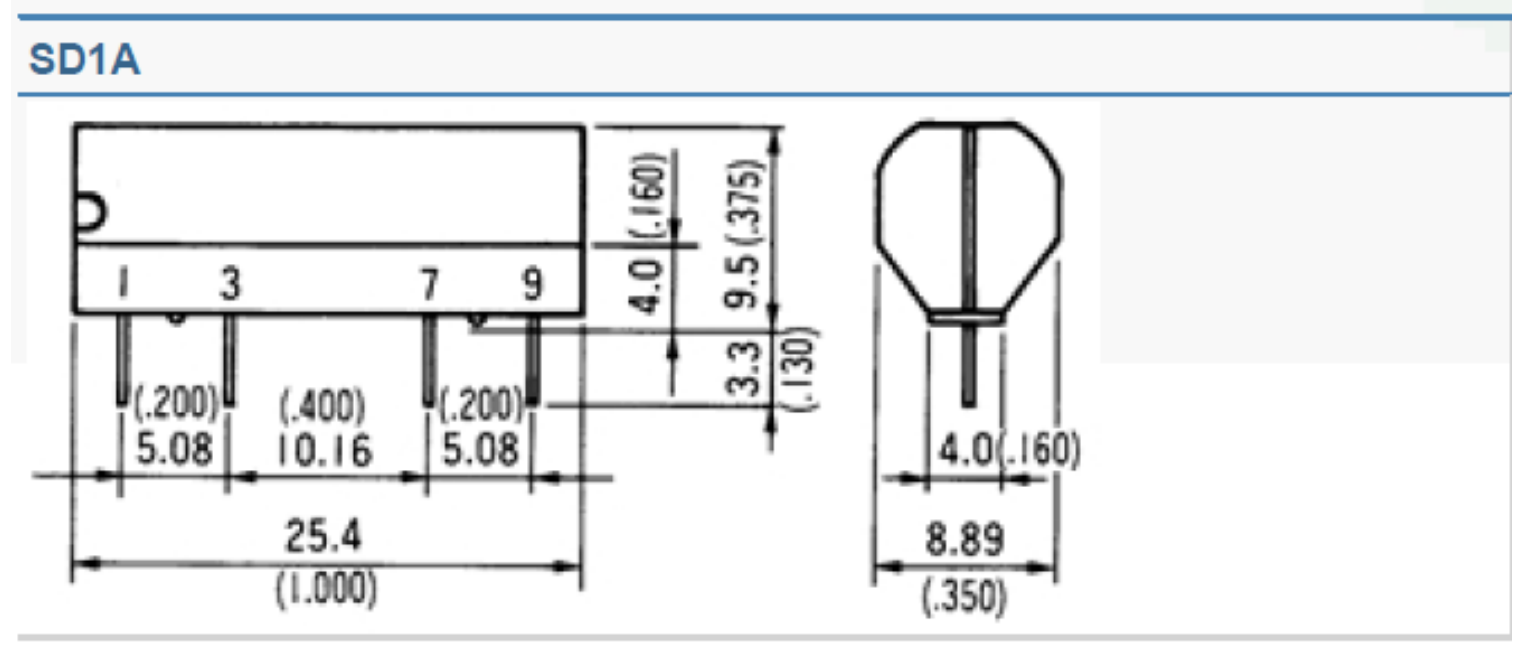




\section{APPENDIX C}

\section{PMMA Fiber Characterization Data}

The following data is the fibers' diameter measured using SEM. In these tables, each fiber was measured three times: two were around $200 \mu \mathrm{m}$ away from the initiating and terminating droplet, one was at the middle location of the fiber to make sure the fiber was uniform. The experimental controlling factors including polymer solutions concentration, dispense time, feed rates, and need gauge were given before each data set.

In the data set, zero means the fiber was broken. And yields were calculated at the end of each data set. 


\begin{tabular}{|c|c|c|c|c|c|c|}
\hline Concentration & $23 \%$ & & & & & \\
\hline Feed rate & $750 \mathrm{~mm} / \mathrm{s}$ & & & & & \\
\hline Needle Gauge & 32 & & & & & \\
\hline $\begin{array}{c}\text { Robot /Valve } \\
\text { Dispense time }\end{array}$ & $0.5 \mathrm{~s} / 0.015 \mathrm{~s}$ & & & & & \\
\hline Fiber \# & $\begin{array}{c}\text { Length } \\
(\mathrm{mm})\end{array}$ & $\begin{array}{c}\text { Leading } \\
\text { Diameter } \\
(\mu \mathrm{m})\end{array}$ & $\begin{array}{c}\text { Middle } \\
\text { Diameter } \\
(\mu \mathrm{m})\end{array}$ & $\begin{array}{c}\text { Terminating } \\
\text { Diameter } \\
(\mu \mathrm{m})\end{array}$ & $\begin{array}{c}\text { Average } \\
\text { Diameter } \\
(\mu \mathrm{m})\end{array}$ & $\begin{array}{c}\text { Standard } \\
\text { Deviation } \\
(\mu \mathrm{m})\end{array}$ \\
\hline 1 & 5 & 0 & 0 & 0 & 0.00 & 0.00 \\
\hline 2 & 10 & 7.617 & 6.421 & 37.09 & 17.04 & 14.18 \\
\hline 3 & 15 & 10.31 & 7.388 & 21.24 & 12.98 & 5.96 \\
\hline 4 & 15 & 8.985 & 6.512 & 39.28 & 18.26 & 14.90 \\
\hline 5 & 20 & 3.395 & 5.194 & 46.17 & 18.25 & 19.75 \\
\hline 6 & 20 & 7.933 & 5.337 & 12.83 & 8.70 & 3.11 \\
\hline 7 & 30 & 18.68 & 5.217 & 9.991 & 11.30 & 5.57 \\
\hline 8 & 30 & 0 & 0 & 0 & 0.00 & 0.00 \\
\hline 9 & 35 & 7.34 & 5.249 & 17.55 & 10.05 & 5.37 \\
\hline 10 & 35 & 1.204 & 3.804 & 11.63 & 5.55 & 4.43 \\
\hline 11 & 40 & 5.67 & 2.911 & 13.07 & 7.22 & 4.29 \\
\hline 12 & 40 & 0 & 0 & 0 & 0.00 & 0.00 \\
\hline Yield & $75 \%$ & & & & & \\
\hline
\end{tabular}

\begin{tabular}{|c|c|c|c|c|l|l|}
\hline Concentration & $23 \%$ & & & & & \\
\hline Feed rate & $750 \mathrm{~mm} / \mathrm{s}$ & & & & & \\
\hline Needle Gauge & 30 & & & & & \\
\hline $\begin{array}{c}\text { Robot /Valve } \\
\text { Dispense time }\end{array}$ & $0.5 \mathrm{~s} / 0.015 \mathrm{~s}$ & & & & & \\
\hline Fiber \# & $\begin{array}{c}\text { Length } \\
(\mathrm{mm})\end{array}$ & $\begin{array}{c}\text { Leading } \\
\text { Diameter } \\
(\mu \mathrm{m})\end{array}$ & $\begin{array}{c}\text { Middle } \\
\text { Diameter } \\
(\mu \mathrm{m})\end{array}$ & $\begin{array}{c}\text { Terminating } \\
\text { Diameter } \\
(\mu \mathrm{m})\end{array}$ & $\begin{array}{c}\text { Average } \\
\text { Diameter } \\
(\mu \mathrm{m})\end{array}$ & $\begin{array}{c}\text { Standard } \\
\text { Deviation } \\
(\mu \mathrm{m})\end{array}$ \\
\hline 1 & 5 & 83.91 & 30.73 & 46.48 & 53.71 & 22.30 \\
\hline 2 & 10 & 27.53 & 9.786 & 39 & 25.44 & 12.02 \\
\hline 3 & 15 & 13.59 & 7.169 & 40.84 & 20.53 & 14.60 \\
\hline 4 & 15 & 8.763 & 8.456 & 40.71 & 19.31 & 15.13 \\
\hline 5 & 20 & 11.68 & 5.234 & 25.28 & 14.06 & 8.36 \\
\hline 6 & 20 & 34.36 & 7.143 & 7.772 & 16.43 & 12.68 \\
\hline 7 & 30 & 10.14 & 5.886 & 27.38 & 14.47 & 9.29 \\
\hline 8 & 30 & 36.26 & 6.904 & 9.548 & 17.57 & 13.26 \\
\hline 9 & 35 & 20.3 & 6.283 & 7.852 & 11.48 & 6.27 \\
\hline 10 & 35 & 25.48 & 5.688 & 1.261 & 10.81 & 10.53 \\
\hline 11 & 40 & 16.03 & 5.347 & 42.79 & 21.39 & 15.75 \\
\hline 12 & 40 & 14.16 & 4.478 & 23.37 & 14.00 & 7.71 \\
\hline Yield & $100 \%$ & & & & & \\
\hline
\end{tabular}




\begin{tabular}{|c|c|c|c|c|c|c|}
\hline Concentration & $23 \%$ & & & & & \\
\hline Feed rate & $750 \mathrm{~mm} / \mathrm{s}$ & & & & & \\
\hline Needle Gauge & 27 & & & & & \\
\hline $\begin{array}{c}\text { Robot /Valve } \\
\text { Dispense time }\end{array}$ & $0.5 \mathrm{~s} / 0.015 \mathrm{~s}$ & & & & & \\
\hline Fiber \# & $\begin{array}{c}\text { Length } \\
(\mathrm{mm})\end{array}$ & $\begin{array}{c}\text { Leading } \\
\text { Diameter } \\
(\mu \mathrm{m})\end{array}$ & $\begin{array}{c}\text { Middle } \\
\text { Diameter } \\
(\mu \mathrm{m})\end{array}$ & $\begin{array}{c}\text { Terminating } \\
\text { Diameter } \\
(\mu \mathrm{m})\end{array}$ & $\begin{array}{c}\text { Average } \\
\text { Diameter } \\
(\mu \mathrm{m})\end{array}$ & $\begin{array}{c}\text { Standard } \\
\text { Deviation } \\
(\mu \mathrm{m})\end{array}$ \\
\hline 1 & 5 & 27.15 & 17.62 & 35.51 & 26.76 & 7.31 \\
\hline 2 & 10 & 29.38 & 11.35 & 40.18 & 26.97 & 11.89 \\
\hline 3 & 15 & 10.36 & 13.41 & 15.76 & 13.18 & 2.21 \\
\hline 4 & 15 & 35.6 & 11.9 & 35.27 & 27.59 & 11.10 \\
\hline 5 & 20 & 11.63 & 9.356 & 12.82 & 11.27 & 1.44 \\
\hline 6 & 20 & 15.94 & 8.084 & 20.2 & 14.74 & 5.02 \\
\hline 7 & 30 & 5.963 & 5.349 & 23.25 & 11.52 & 8.30 \\
\hline 8 & 30 & 10.53 & 5.282 & 28.87 & 14.89 & 10.11 \\
\hline 9 & 35 & 7.444 & 3.08 & 17.18 & 9.23 & 5.89 \\
\hline 10 & 35 & 42.31 & 6.589 & 26.03 & 24.98 & 14.60 \\
\hline 11 & 40 & 23.22 & 6.568 & 12.33 & 14.04 & 6.90 \\
\hline 12 & 40 & 7.066 & 4.472 & 27.73 & 13.09 & 10.41 \\
\hline Yield & $100 \%$ & & & & & \\
\hline
\end{tabular}

\begin{tabular}{|c|c|c|c|c|c|c|}
\hline Concentration & $23 \%$ & & & & & \\
\hline $\begin{array}{c}\text { Feed rate } \\
\text { Needle Gauge }\end{array}$ & $250 \mathrm{~mm} / \mathrm{s}$ & & & & & \\
\hline $\begin{array}{c}\text { Robot /Valve } \\
\text { Dispense time }\end{array}$ & $0.5 \mathrm{~s} / 0.015 \mathrm{~s}$ & & & & & \\
\hline Fiber \# & $\begin{array}{c}\text { Length } \\
(\mathrm{mm})\end{array}$ & $\begin{array}{c}\text { Leading } \\
\text { Diameter } \\
(\mu \mathrm{m})\end{array}$ & $\begin{array}{c}\text { Middle } \\
\text { Diameter } \\
(\mu \mathrm{m})\end{array}$ & $\begin{array}{c}\text { Terminating } \\
\text { Diameter } \\
(\mu \mathrm{m})\end{array}$ & $\begin{array}{c}\text { Average } \\
\text { Diameter } \\
(\mu \mathrm{m})\end{array}$ & $\begin{array}{c}\text { Standard } \\
\text { Deviation } \\
(\mu \mathrm{m})\end{array}$ \\
\hline 1 & 5 & 74.52 & 62.77 & 120.6 & 85.96 & 24.96 \\
\hline 2 & 10 & 2.627 & 7.066 & 14.53 & 8.07 & 4.91 \\
\hline 3 & 15 & 51.11 & 24.95 & 58.09 & 44.72 & 14.26 \\
\hline 4 & 15 & 0.1676 & 8.948 & 21.04 & 10.05 & 8.56 \\
\hline 5 & 20 & 14.21 & 11.36 & 40.3 & 21.96 & 13.02 \\
\hline 6 & 20 & 26.18 & 8.718 & 7.968 & 14.29 & 8.41 \\
\hline 7 & 30 & 12.42 & 7.397 & 32.07 & 17.30 & 10.65 \\
\hline 8 & 30 & 2.825 & 6.508 & 18.18 & 9.17 & 6.55 \\
\hline 9 & 35 & 8.467 & 6.347 & 22.74 & 12.52 & 7.28 \\
\hline 10 & 35 & 12.11 & 7.961 & 29.52 & 16.53 & 9.34 \\
\hline 11 & 40 & 12.19 & 6.724 & 6.047 & 8.32 & 2.75 \\
\hline 12 & 40 & 10.14 & 7.518 & 42.32 & 19.99 & 15.82 \\
\hline Yield & $100 \%$ & & & & & \\
\hline
\end{tabular}




\begin{tabular}{|c|c|c|c|c|c|c|}
\hline Concentration & $23 \%$ & & & & & \\
\hline Feed rate & $450 \mathrm{~mm} / \mathrm{s}$ & & & & & \\
\hline Needle Gauge & 32 & & & & & \\
\hline $\begin{array}{c}\text { Robot /Valve } \\
\text { Dispense time }\end{array}$ & $0.5 \mathrm{~s} / 0.015 \mathrm{~s}$ & & & & & \\
\hline Fiber \# & $\begin{array}{c}\text { Length } \\
(\mathrm{mm})\end{array}$ & $\begin{array}{c}\text { Leading } \\
\text { Diameter } \\
(\mu \mathrm{m})\end{array}$ & $\begin{array}{c}\text { Middle } \\
\text { Diameter } \\
(\mu \mathrm{m})\end{array}$ & $\begin{array}{c}\text { Terminating } \\
\text { Diameter } \\
(\mu \mathrm{m})\end{array}$ & $\begin{array}{c}\text { Average } \\
\text { Diameter } \\
(\mu \mathrm{m})\end{array}$ & $\begin{array}{c}\text { Standard } \\
\text { Deviation } \\
(\mu \mathrm{m})\end{array}$ \\
\hline 1 & 5 & 0 & 0 & 0 & 0.00 & 0.00 \\
\hline 2 & 10 & 26.76 & 9.576 & 27.7 & 21.35 & 8.33 \\
\hline 3 & 15 & 23.51 & 6.443 & 20.68 & 16.88 & 7.47 \\
\hline 4 & 15 & 6.233 & 8.677 & 18.48 & 11.13 & 5.29 \\
\hline 5 & 20 & 6.507 & 5.857 & 25.5 & 12.62 & 9.11 \\
\hline 6 & 20 & 2.852 & 6.828 & 32.02 & 13.90 & 12.92 \\
\hline 7 & 30 & 4.305 & 3.805 & 18.3 & 8.80 & 6.72 \\
\hline 8 & 30 & 0 & 0 & 0 & 0.00 & 0.00 \\
\hline 9 & 35 & 10.77 & 5.276 & 24.08 & 13.38 & 7.89 \\
\hline 10 & 35 & 0 & 0 & 0 & 0.00 & 0.00 \\
\hline 11 & 40 & 0 & 0 & 0 & 0.00 & 0.00 \\
\hline 12 & 40 & 0 & 0 & 0 & 0.00 & 0.00 \\
\hline Yield & $58.33 \%$ & & & & & \\
\hline
\end{tabular}

\begin{tabular}{|c|c|c|c|c|c|c|}
\hline Concentration & $23 \%$ & & & & & \\
\hline Feed rate & $525 \mathrm{~mm} / \mathrm{s}$ & & & & & \\
\hline $\begin{array}{c}\text { Needle Gauge } \\
\text { Robot /Valve } \\
\text { Dispense time }\end{array}$ & $0.5 \mathrm{~s} / 0.015 \mathrm{~s}$ & & & & & \\
\hline Fiber \# & $\begin{array}{c}\text { Length } \\
(\mathrm{mm})\end{array}$ & $\begin{array}{c}\text { Leading } \\
\text { Diameter } \\
(\mu \mathrm{m})\end{array}$ & $\begin{array}{c}\text { Middle } \\
\text { Diameter } \\
(\mu \mathrm{m})\end{array}$ & $\begin{array}{c}\text { Terminating } \\
\text { Diameter } \\
(\mu \mathrm{m})\end{array}$ & $\begin{array}{c}\text { Average } \\
\text { Diameter } \\
(\mu \mathrm{m})\end{array}$ & $\begin{array}{c}\text { Standard } \\
\text { Deviation } \\
(\mu \mathrm{m})\end{array}$ \\
\hline 1 & 5 & 0 & 0 & 0 & 0.00 & 0.00 \\
\hline 2 & 10 & 10.68 & 5.754 & 22.85 & 13.09 & 7.19 \\
\hline 3 & 15 & 40.07 & 7.183 & 42.42 & 29.89 & 16.09 \\
\hline 4 & 15 & 17.52 & 11.35 & 41.9 & 23.59 & 13.19 \\
\hline 5 & 20 & 10.05 & 5.974 & 23.41 & 13.14 & 7.45 \\
\hline 6 & 20 & 4.186 & 5.491 & 25.85 & 11.84 & 9.92 \\
\hline 7 & 30 & 12.15 & 5.022 & 7.825 & 8.33 & 2.93 \\
\hline 8 & 30 & 0 & 0 & 0 & 0.00 & 0.00 \\
\hline 9 & 35 & 14.39 & 6.003 & 13.59 & 11.33 & 3.78 \\
\hline 10 & 35 & 8.396 & 9.213 & 14.67 & 10.76 & 2.79 \\
\hline 11 & 40 & 12.78 & 4.295 & 17.86 & 11.65 & 5.60 \\
\hline 12 & 40 & 0 & 0 & 0 & 0.00 & 0.00 \\
\hline Yield & $75 \%$ & & & & & \\
\hline Concentration & $23 \%$ & & & & & \\
\hline
\end{tabular}




\begin{tabular}{|c|c|c|c|c|c|c|}
\hline Feed rate & $600 \mathrm{~mm} / \mathrm{s}$ & & & & & \\
\hline Needle Gauge & 32 & & & & & \\
\hline $\begin{array}{l}\text { Robot /Valve } \\
\text { Dispense time }\end{array}$ & $0.5 \mathrm{~s} / 0.015 \mathrm{~s}$ & & & & & \\
\hline Fiber \# & $\begin{array}{c}\text { Length } \\
(\mathrm{mm})\end{array}$ & $\begin{array}{c}\text { Leading } \\
\text { Diameter } \\
(\mu \mathrm{m})\end{array}$ & $\begin{array}{c}\text { Middle } \\
\text { Diameter } \\
(\mu \mathrm{m})\end{array}$ & $\begin{array}{c}\text { Terminating } \\
\text { Diameter } \\
(\mu \mathrm{m})\end{array}$ & $\begin{array}{c}\text { Average } \\
\text { Diameter } \\
(\mu \mathrm{m})\end{array}$ & $\begin{array}{c}\text { Standard } \\
\text { Deviation } \\
(\mu \mathrm{m})\end{array}$ \\
\hline 1 & 5 & 23.43 & 11.84 & 12.21 & 15.83 & 5.38 \\
\hline 2 & 10 & 15.87 & 8.797 & 21.6 & 15.42 & 5.24 \\
\hline 3 & 15 & 23.3 & 8.549 & 7.67 & 13.17 & 7.17 \\
\hline 4 & 15 & 10.14 & 5.73 & 16.36 & 10.74 & 4.36 \\
\hline 5 & 20 & 76.62 & 3.44 & 25.61 & 35.22 & 30.64 \\
\hline 6 & 20 & 22.24 & 9.621 & 15.53 & 15.80 & 5.16 \\
\hline 7 & 30 & 8.769 & 3.791 & 31.51 & 14.69 & 12.07 \\
\hline 8 & 30 & 0 & 0 & 0 & 0.00 & 0.00 \\
\hline 9 & 35 & 6.324 & 5.235 & 12.5 & 8.02 & 3.20 \\
\hline 10 & 35 & 13.34 & 5.308 & 21.72 & 13.46 & 6.70 \\
\hline 11 & 40 & 15.9 & 5.831 & 31.69 & 17.81 & 10.64 \\
\hline 12 & 40 & 0 & 0 & 0 & 0.00 & 0.00 \\
\hline Yield & $83.3 \%$ & & & & & \\
\hline
\end{tabular}

\begin{tabular}{|c|c|c|c|c|c|c|}
\hline Concentration & $23 \%$ & & & & & \\
\hline Feed rate & $675 \mathrm{~mm} / \mathrm{s}$ & & & & & \\
\hline Needle Gauge & 32 & & & & & \\
\hline $\begin{array}{c}\text { Robot /Valve } \\
\text { Dispense time }\end{array}$ & $0.5 \mathrm{~s} / 0.015 \mathrm{~s}$ & & & & & \\
\hline Fiber \# & $\begin{array}{c}\text { Length } \\
(\mathrm{mm})\end{array}$ & $\begin{array}{c}\text { Leading } \\
\text { Diameter } \\
(\mu \mathrm{m})\end{array}$ & $\begin{array}{c}\text { Middle } \\
\text { Diameter } \\
(\mu \mathrm{m})\end{array}$ & $\begin{array}{c}\text { Terminating } \\
\text { Diameter } \\
(\mu \mathrm{m})\end{array}$ & $\begin{array}{c}\text { Average } \\
\text { Diameter } \\
(\mu \mathrm{m})\end{array}$ & $\begin{array}{c}\text { Standard } \\
\text { Deviation } \\
(\mu \mathrm{m})\end{array}$ \\
\hline 1 & 5 & 0 & 0 & 0 & 0.00 & 0.00 \\
\hline 2 & 10 & 0 & 0 & 0 & 0.00 & 0.00 \\
\hline 3 & 15 & 8.602 & 4.59 & 6.136 & 6.44 & 1.65 \\
\hline 4 & 15 & 7.045 & 6.062 & 22.92 & 12.01 & 7.73 \\
\hline 5 & 20 & 24.13 & 4.21 & 6.19 & 11.51 & 8.96 \\
\hline 6 & 20 & 28.26 & 7.472 & 10.52 & 15.42 & 9.17 \\
\hline 7 & 30 & 28.58 & 7.595 & 3.673 & 13.28 & 10.93 \\
\hline 8 & 30 & 24.68 & 4.834 & 2.481 & 10.67 & 9.96 \\
\hline 9 & 35 & 30.94 & 3.266 & 2.997 & 12.40 & 13.11 \\
\hline 10 & 35 & 20.74 & 4.155 & 1.564 & 8.82 & 8.50 \\
\hline 11 & 40 & 8.73 & 4.358 & 37.26 & 16.78 & 14.59 \\
\hline 12 & 40 & 0 & 0 & 0 & 0.00 & 0.00 \\
\hline Yield & $75 \%$ & & & & & \\
\hline
\end{tabular}




\begin{tabular}{|c|c|c|c|c|c|c|}
\hline Concentration & $23 \%$ & & & & & \\
\hline Feed rate & $750 \mathrm{~mm} / \mathrm{s}$ & & & & & \\
\hline Needle Gauge & 32 & & & & & \\
\hline $\begin{array}{c}\text { Robot /Valve } \\
\text { Dispense time }\end{array}$ & $0.5 \mathrm{~s} / 0.015 \mathrm{~s}$ & & & & & \\
\hline Fiber \# & $\begin{array}{c}\text { Length } \\
(\mathrm{mm})\end{array}$ & $\begin{array}{c}\text { Leading } \\
\text { Diameter } \\
(\mu \mathrm{m})\end{array}$ & $\begin{array}{c}\text { Middle } \\
\text { Diameter } \\
(\mu \mathrm{m})\end{array}$ & $\begin{array}{c}\text { Terminating } \\
\text { Diameter } \\
(\mu \mathrm{m})\end{array}$ & $\begin{array}{c}\text { Average } \\
\text { Diameter } \\
(\mu \mathrm{m})\end{array}$ & $\begin{array}{c}\text { Standard } \\
\text { Deviation } \\
(\mu \mathrm{m})\end{array}$ \\
\hline 1 & 5 & 0 & 0 & 0 & 0.00 & 0.00 \\
\hline 2 & 10 & 7.617 & 6.421 & 37.09 & 17.04 & 14.18 \\
\hline 3 & 15 & 10.31 & 7.388 & 21.24 & 12.98 & 5.96 \\
\hline 4 & 15 & 8.985 & 6.512 & 39.28 & 18.26 & 14.90 \\
\hline 5 & 20 & 3.395 & 5.194 & 46.17 & 18.25 & 19.75 \\
\hline 6 & 20 & 7.933 & 5.337 & 12.83 & 8.70 & 3.11 \\
\hline 7 & 30 & 18.68 & 5.217 & 9.991 & 11.30 & 5.57 \\
\hline 8 & 30 & 0 & 0 & 0 & 0.00 & 0.00 \\
\hline 9 & 35 & 7.34 & 5.249 & 17.55 & 10.05 & 5.37 \\
\hline 10 & 35 & 1.204 & 3.804 & 11.63 & 5.55 & 4.43 \\
\hline 11 & 40 & 5.67 & 2.911 & 13.07 & 7.22 & 4.29 \\
\hline 12 & 40 & 0 & 0 & 0 & 0.00 & 0.00 \\
\hline Yield & $75 \%$ & & & & & \\
\hline
\end{tabular}

\begin{tabular}{|c|c|c|c|c|c|c|}
\hline Concentration & $23 \%$ & & & & & \\
\hline Feed rate & $\begin{array}{c}750 \\
\mathrm{~mm} / \mathrm{s}\end{array}$ & & & & & \\
\hline Needle Gauge & 32 & & & & & \\
\hline $\begin{array}{c}\text { Robot /Valve } \\
\text { Dispense time }\end{array}$ & $1 \mathrm{~s} / 1 \mathrm{~s}$ & & & & & \\
\hline Fiber \# & $\begin{array}{c}\text { Length } \\
(\mathrm{mm})\end{array}$ & $\begin{array}{c}\text { Leading } \\
\text { Diameter } \\
(\mu \mathrm{m})\end{array}$ & $\begin{array}{c}\text { Middle } \\
\text { Diameter } \\
(\mu \mathrm{m})\end{array}$ & $\begin{array}{c}\text { Terminating } \\
\text { Diameter } \\
(\mu \mathrm{m})\end{array}$ & $\begin{array}{c}\text { Average } \\
\text { Diameter } \\
(\mu \mathrm{m})\end{array}$ & $\begin{array}{c}\text { Standard } \\
\text { Deviation } \\
(\mu \mathrm{m})\end{array}$ \\
\hline 1 & 5 & 2.809 & 1.601 & 1.173 & 1.86 & 0.69 \\
\hline 2 & 10 & 4.497 & 6.028 & 17.69 & 9.41 & 5.89 \\
\hline 3 & 15 & 3.415 & 6.18 & 26.25 & 11.95 & 10.18 \\
\hline 4 & 15 & 0.5017 & 4.704 & 15.5 & 6.90 & 6.32 \\
\hline 5 & 20 & 0.8315 & 8.355 & 10.41 & 6.53 & 4.12 \\
\hline 6 & 20 & 3.032 & 4.037 & 22.02 & 9.70 & 8.72 \\
\hline 7 & 30 & 3.426 & 3.771 & 19.93 & 9.04 & 7.70 \\
\hline 8 & 30 & 2.484 & 3.911 & 7.778 & 4.72 & 2.24 \\
\hline 9 & 35 & 9.269 & 3.944 & 5.404 & 6.21 & 2.25 \\
\hline 10 & 35 & 5.932 & 3.039 & 14.95 & 7.97 & 5.07 \\
\hline 11 & 40 & 6.787 & 3.246 & 15.03 & 8.35 & 4.94 \\
\hline 12 & 40 & 11.04 & 5.283 & 2.18 & 6.17 & 3.67 \\
\hline Yield & $100 \%$ & & & & & \\
\hline & & & & & & \\
\hline
\end{tabular}




\begin{tabular}{|c|c|c|c|c|c|c|}
\hline Concentration & $23 \%$ & & & & & \\
\hline Feed rate & $750 \mathrm{~mm} / \mathrm{s}$ & & & & & \\
\hline Needle Gauge & 32 & & & & & \\
\hline $\begin{array}{c}\text { Robot /Valve } \\
\text { Dispense time }\end{array}$ & $0.05 \mathrm{~s} / 0.05 \mathrm{~s}$ & & & & & \\
\hline Fiber \# & $\begin{array}{c}\text { Length } \\
(\mathrm{mm})\end{array}$ & $\begin{array}{c}\text { Leading } \\
\text { Diameter } \\
(\mu \mathrm{m})\end{array}$ & $\begin{array}{c}\text { Middle } \\
\text { Diameter } \\
(\mu \mathrm{m})\end{array}$ & $\begin{array}{c}\text { Terminating } \\
\text { Diameter } \\
(\mu \mathrm{m})\end{array}$ & $\begin{array}{c}\text { Average } \\
\text { Diameter } \\
(\mu \mathrm{m})\end{array}$ & $\begin{array}{c}\text { Standard } \\
\text { Deviation } \\
(\mu \mathrm{m})\end{array}$ \\
\hline 1 & 5 & 0 & 0 & 0 & 0.00 & 0.00 \\
\hline 2 & 10 & 39.17 & 16.59 & 39.49 & 31.75 & 10.72 \\
\hline 3 & 15 & 12.16 & 6.93 & 15.87 & 11.65 & 3.67 \\
\hline 4 & 15 & 0.3088 & 3.094 & 3.261 & 2.22 & 1.35 \\
\hline 5 & 20 & 0.5744 & 3.442 & 6.313 & 3.44 & 2.34 \\
\hline 6 & 20 & 0.1848 & 4.107 & 9.712 & 4.67 & 3.91 \\
\hline 7 & 30 & 2.022 & 2.624 & 4.259 & 2.97 & 0.95 \\
\hline 8 & 30 & 1.013 & 3.68 & 5.866 & 3.52 & 1.98 \\
\hline 9 & 35 & 3.554 & 3.836 & 8.261 & 5.22 & 2.16 \\
\hline 10 & 35 & 3.427 & 3.047 & 5.688 & 4.05 & 1.17 \\
\hline 11 & 40 & 3.386 & 3.471 & 6.972 & 4.61 & 1.67 \\
\hline 12 & 40 & 1.785 & 1.313 & 6.452 & 3.18 & 2.32 \\
\hline Yield & $91.7 \%$ & & & & & \\
\hline
\end{tabular}

\begin{tabular}{|c|c|c|c|c|c|c|}
\hline Concentration & $23 \%$ & & & & & \\
\hline Feed rate & $750 \mathrm{~mm} / \mathrm{s}$ & & & & & \\
\hline Needle Gauge & 32 & & & & & \\
\hline $\begin{array}{l}\text { Robot /Valve } \\
\text { Dispense time }\end{array}$ & $0.02 \mathrm{~s} / 0.02 \mathrm{~s}$ & & & & & \\
\hline Fiber \# & $\begin{array}{l}\text { Length } \\
(\mathrm{mm})\end{array}$ & $\begin{array}{c}\text { Leading } \\
\text { Diameter } \\
(\mu \mathrm{m})\end{array}$ & $\begin{array}{c}\text { Middle } \\
\text { Diameter } \\
(\mu \mathrm{m})\end{array}$ & $\begin{array}{c}\text { Terminatin } \\
\text { g Diameter } \\
(\mu \mathrm{m})\end{array}$ & $\begin{array}{c}\text { Average } \\
\text { Diameter } \\
(\mu \mathrm{m})\end{array}$ & $\begin{array}{c}\text { Standard } \\
\text { Deviation } \\
(\mu \mathrm{m})\end{array}$ \\
\hline 1 & 5 & 9.031 & 8.631 & 42.52 & 20.06 & 15.88 \\
\hline 2 & 10 & 18.41 & 6.378 & 22.62 & 15.80 & 6.88 \\
\hline 3 & 15 & 24.94 & 7.031 & 24.33 & 18.77 & 8.30 \\
\hline 4 & 15 & 6.87 & 3.827 & 13.67 & 8.12 & 4.11 \\
\hline 5 & 20 & 27.26 & 7.25 & 18.49 & 17.67 & 8.19 \\
\hline 6 & 20 & 7.09 & 5.73 & 24.09 & 12.30 & 8.35 \\
\hline 7 & 30 & 0 & 0 & 0 & 0.00 & 0.00 \\
\hline 8 & 30 & 13.71 & 2.442 & 27.13 & 14.43 & 10.09 \\
\hline 9 & 35 & 31.42 & 4.967 & 26.95 & 21.11 & 11.56 \\
\hline 10 & 35 & 0 & 0 & 0 & 0.00 & 0.00 \\
\hline 11 & 40 & 10.21 & 2.985 & 19.56 & 10.92 & 6.79 \\
\hline 12 & 40 & 0 & 0 & 0 & 0.00 & 0.00 \\
\hline Yield & $75 \%$ & & & & & \\
\hline
\end{tabular}




\begin{tabular}{|c|c|c|c|c|c|c|}
\hline Concentration & $23 \%$ & & & & & \\
\hline Feed rate & $750 \mathrm{~mm} / \mathrm{s}$ & & & & & \\
\hline Needle Gauge & 32 & & & & & \\
\hline $\begin{array}{l}\text { Robot /Valve } \\
\text { Dispense time }\end{array}$ & $0.02 \mathrm{~s} / 0.02 \mathrm{~s}$ & & & & & \\
\hline Fiber \# & $\begin{array}{c}\text { Length } \\
(\mathrm{mm})\end{array}$ & $\begin{array}{c}\text { Leading } \\
\text { Diameter } \\
(\mu \mathrm{m})\end{array}$ & $\begin{array}{c}\text { Middle } \\
\text { Diameter } \\
(\mu \mathrm{m})\end{array}$ & $\begin{array}{c}\text { Terminating } \\
\text { Diameter } \\
(\mu \mathrm{m})\end{array}$ & $\begin{array}{c}\text { Average } \\
\text { Diameter } \\
(\mu \mathrm{m})\end{array}$ & $\begin{array}{c}\text { Standard } \\
\text { Deviation } \\
(\mu \mathrm{m})\end{array}$ \\
\hline 1 & 5 & 9.031 & 8.631 & 42.52 & 20.06 & 15.88 \\
\hline 2 & 10 & 18.41 & 6.378 & 22.62 & 15.80 & 6.88 \\
\hline 3 & 15 & 24.94 & 7.031 & 24.33 & 18.77 & 8.30 \\
\hline 4 & 15 & 6.87 & 3.827 & 13.67 & 8.12 & 4.11 \\
\hline 5 & 20 & 27.26 & 7.25 & 18.49 & 17.67 & 8.19 \\
\hline 6 & 20 & 7.09 & 5.73 & 24.09 & 12.30 & 8.35 \\
\hline 7 & 30 & 0 & 0 & 0 & 0.00 & 0.00 \\
\hline 8 & 30 & 13.71 & 2.442 & 27.13 & 14.43 & 10.09 \\
\hline 9 & 35 & 31.42 & 4.967 & 26.95 & 21.11 & 11.56 \\
\hline 10 & 35 & 0 & 0 & 0 & 0.00 & 0.00 \\
\hline 11 & 40 & 10.21 & 2.985 & 19.56 & 10.92 & 6.79 \\
\hline 12 & 40 & 0 & 0 & 0 & 0.00 & 0.00 \\
\hline Yield & $75 \%$ & & & & & \\
\hline
\end{tabular}

\begin{tabular}{|c|c|c|c|c|c|c|}
\hline Concentration & $24 \%$ & & & & & \\
\hline Feed rate & $750 \mathrm{~mm} / \mathrm{s}$ & & & & & \\
\hline Needle Gauge & 32 & & & & & \\
\hline $\begin{array}{l}\text { Robot /Valve } \\
\text { Dispense time }\end{array}$ & $\begin{array}{c}0.02 s / 0.02 \\
s\end{array}$ & & & & & \\
\hline Fiber \# & $\begin{array}{c}\text { Length } \\
(\mathrm{mm})\end{array}$ & $\begin{array}{c}\text { Leading } \\
\text { Diameter } \\
(\mu \mathrm{m})\end{array}$ & $\begin{array}{c}\text { Middle } \\
\text { Diameter } \\
(\mu \mathrm{m})\end{array}$ & $\begin{array}{c}\text { Terminating } \\
\text { Diameter } \\
(\mu \mathrm{m})\end{array}$ & $\begin{array}{c}\text { Average } \\
\text { Diameter } \\
(\mu \mathrm{m})\end{array}$ & $\begin{array}{c}\text { Standard } \\
\text { Deviation } \\
(\mu \mathrm{m})\end{array}$ \\
\hline 1 & 5 & 0 & 0 & 0 & 0.00 & 0.00 \\
\hline 2 & 10 & 11.56 & 5.978 & 17.01 & 11.52 & 4.50 \\
\hline 3 & 15 & 1.866 & 2.887 & 1.948 & 2.23 & 0.46 \\
\hline 4 & 15 & 0.6228 & 2.871 & 7.062 & 3.52 & 2.67 \\
\hline 5 & 20 & 1.55 & 2.53 & 4.727 & 2.94 & 1.33 \\
\hline 6 & 20 & 1.947 & 3.557 & 5.873 & 3.79 & 1.61 \\
\hline 7 & 30 & 2.022 & 1.841 & 3.047 & 2.30 & 0.53 \\
\hline 8 & 30 & 2.624 & 1.882 & 3.138 & 2.55 & 0.52 \\
\hline 9 & 35 & 4.997 & 0.5707 & 3.913 & 3.16 & 1.88 \\
\hline 10 & 35 & 0 & 0 & 0 & 0.00 & 0.00 \\
\hline 11 & 40 & 0.4153 & 1.426 & 2.297 & 1.38 & 0.77 \\
\hline 12 & 40 & 4.409 & 4.098 & 2.869 & 3.79 & 0.66 \\
\hline Yield & $91.7 \%$ & & & & & \\
\hline Concentration & $25 \%$ & & & & & \\
\hline
\end{tabular}




\begin{tabular}{|c|c|c|c|c|c|c|}
\hline Feed rate & $750 \mathrm{~mm} / \mathrm{s}$ & & & & & \\
\hline Needle Gauge & 32 & & & & & \\
\hline $\begin{array}{l}\text { Robot / Valve } \\
\text { Dispense time }\end{array}$ & $\begin{array}{c}0.02 \mathrm{~s} / 0.02 \\
\mathrm{~s}\end{array}$ & & & & & \\
\hline Fiber \# & $\begin{array}{c}\text { Length } \\
(\mathrm{mm})\end{array}$ & $\begin{array}{c}\text { Leading } \\
\text { Diameter } \\
(\mu \mathrm{m})\end{array}$ & $\begin{array}{c}\text { Middle } \\
\text { Diameter } \\
(\mu \mathrm{m})\end{array}$ & $\begin{array}{c}\text { Terminating } \\
\text { Diameter } \\
(\mu \mathrm{m})\end{array}$ & $\begin{array}{c}\text { Average } \\
\text { Diameter } \\
(\mu \mathrm{m})\end{array}$ & $\begin{array}{c}\text { Standard } \\
\text { Deviation } \\
(\mu \mathrm{m})\end{array}$ \\
\hline 1 & 5 & 0 & 0 & 0 & 0.00 & 0.00 \\
\hline 2 & 10 & 19.02 & 4.842 & 12.54 & 12.13 & 5.80 \\
\hline 3 & 15 & 2.227 & 4.226 & 18.55 & 8.33 & 7.27 \\
\hline 4 & 15 & 1.757 & 4.98 & 12.09 & 6.28 & 4.32 \\
\hline 5 & 20 & 13.04 & 0.6798 & 0.8023 & 4.84 & 5.80 \\
\hline 6 & 20 & 3.271 & 0.7274 & 0.8225 & 1.61 & 1.18 \\
\hline 7 & 30 & 2.377 & 1.114 & 4.104 & 2.53 & 1.23 \\
\hline 8 & 30 & 14.33 & 3.415 & 3.571 & 7.11 & 5.11 \\
\hline 9 & 35 & 18.31 & 1.74 & 5.744 & 8.60 & 7.06 \\
\hline 10 & 35 & 0 & 0 & 0 & 0.00 & 0.00 \\
\hline 11 & 40 & 3.248 & 5.271 & 21.17 & 9.90 & 8.01 \\
\hline 12 & 40 & 1.951 & 5.694 & 9.122 & 5.59 & 2.93 \\
\hline Yield & $83.3 \%$ & & & & & \\
\hline
\end{tabular}

\begin{tabular}{|c|c|c|c|c|c|c|}
\hline Concentration & $26 \%$ & & & & & \\
\hline Feed rate & $750 \mathrm{~mm} / \mathrm{s}$ & & & & & \\
\hline Needle Gauge & 32 & & & & & \\
\hline $\begin{array}{c}\text { Robot /Valve } \\
\text { Dispense time }\end{array}$ & $0.02 \mathrm{~s} / 0.02 \mathrm{~s}$ & & & & & \\
\hline Fiber \# & $\begin{array}{c}\text { Length } \\
(\mathrm{mm})\end{array}$ & $\begin{array}{c}\text { Leading } \\
\text { Diameter } \\
(\mu \mathrm{m})\end{array}$ & $\begin{array}{c}\text { Middle } \\
\text { Diameter } \\
(\mu \mathrm{m})\end{array}$ & $\begin{array}{c}\text { Terminating } \\
\text { Diameter } \\
(\mu \mathrm{m})\end{array}$ & $\begin{array}{c}\text { Average } \\
\text { Diameter } \\
(\mu \mathrm{m})\end{array}$ & $\begin{array}{c}\text { Standard } \\
\text { Deviation } \\
(\mu \mathrm{m})\end{array}$ \\
\hline 1 & 5 & 0 & 0 & 0 & 0.00 & 0.00 \\
\hline 2 & 10 & 32.57 & 10.43 & 14.67 & 19.22 & 9.59 \\
\hline 3 & 15 & 30.96 & 4.634 & 16.39 & 17.33 & 10.77 \\
\hline 4 & 15 & 14.2 & 2.998 & 32.21 & 16.47 & 12.03 \\
\hline 5 & 20 & 12.84 & 7.984 & 15.99 & 12.27 & 3.29 \\
\hline 6 & 20 & 38.58 & 10.29 & 23.77 & 24.21 & 11.55 \\
\hline 7 & 30 & 0 & 0 & 0 & 0.00 & 0.00 \\
\hline 8 & 30 & 27.93 & 8.531 & 52.6 & 29.69 & 18.03 \\
\hline 9 & 35 & 0 & 0 & 0 & 0.00 & 0.00 \\
\hline 10 & 35 & 37.17 & 9.48 & 21.24 & 22.63 & 11.35 \\
\hline 11 & 40 & 28.93 & 15.42 & 18.51 & 20.95 & 5.78 \\
\hline 12 & 40 & 40.99 & 12.45 & 22.02 & 25.15 & 11.86 \\
\hline Yield & $75 \%$ & & & & & \\
\hline
\end{tabular}




\begin{tabular}{|c|c|c|c|c|c|c|}
\hline Concentration & $27 \%$ & & & & & \\
\hline Feed rate & $750 \mathrm{~mm} / \mathrm{s}$ & & & & & \\
\hline Needle Gauge & 32 & & & & & \\
\hline $\begin{array}{c}\text { Robot /Valve } \\
\text { Dispense time }\end{array}$ & $0.02 \mathrm{~s} / 0.02 \mathrm{~s}$ & & & & & \\
\hline Fiber \# & $\begin{array}{c}\text { Length } \\
(\mathrm{mm})\end{array}$ & $\begin{array}{c}\text { Leading } \\
\text { Diameter } \\
(\mu \mathrm{m})\end{array}$ & $\begin{array}{c}\text { Middle } \\
\text { Diameter } \\
(\mu \mathrm{m})\end{array}$ & $\begin{array}{c}\text { Terminating } \\
\text { Diameter } \\
(\mu \mathrm{m})\end{array}$ & $\begin{array}{c}\text { Average } \\
\text { Diameter } \\
(\mu \mathrm{m})\end{array}$ & $\begin{array}{c}\text { Standard } \\
\text { Deviation } \\
(\mu \mathrm{m})\end{array}$ \\
\hline 1 & 5 & 0 & 0 & 0 & 0.00 & 0.00 \\
\hline 2 & 10 & 18.23 & 7.299 & 9.278 & 11.60 & 4.76 \\
\hline 3 & 15 & 14.91 & 6.343 & 28.32 & 16.52 & 9.04 \\
\hline 4 & 15 & 45.58 & 7.786 & 41.16 & 31.51 & 16.87 \\
\hline 5 & 20 & 38.93 & 6.357 & 21.57 & 22.29 & 13.31 \\
\hline 6 & 20 & 33.94 & 7.901 & 23.19 & 21.68 & 10.68 \\
\hline 7 & 30 & 22.26 & 4.317 & 7.125 & 11.23 & 7.88 \\
\hline 8 & 30 & 43.75 & 3.838 & 27.15 & 24.91 & 16.37 \\
\hline 9 & 35 & 46.76 & 6.951 & 52.73 & 35.48 & 20.32 \\
\hline 10 & 35 & 4.911 & 3.521 & 27.46 & 11.96 & 10.97 \\
\hline 11 & 40 & 29.75 & 5.892 & 31.32 & 22.32 & 11.63 \\
\hline 12 & 40 & 40.93 & 5.157 & 48.99 & 31.69 & 19.05 \\
\hline Yield & $91.7 \%$ & & & & & \\
\hline & & & & & & \\
\hline
\end{tabular}




\begin{tabular}{|c|c|c|c|c|c|c|}
\hline Concentration & $23 \%$ & & & & & \\
\hline Feed rate & $\begin{array}{c}22.5 \\
\mathrm{~mm} / \mathrm{s}\end{array}$ & & & & & \\
\hline Needle Gauge & 25 & & & & & \\
\hline Dispense Time & $0.02 s$ & & & & & \\
\hline \multicolumn{7}{|l|}{ Short to Long } \\
\hline Fiber \# & $\begin{array}{l}\text { Length } \\
(\mathrm{mm})\end{array}$ & $\begin{array}{c}\text { Leading } \\
\text { Diameter } \\
(\mu \mathrm{m}) \\
\end{array}$ & $\begin{array}{c}\text { Middle } \\
\text { Diameter } \\
(\mu \mathrm{m})\end{array}$ & $\begin{array}{c}\text { Terminating } \\
\text { Diameter } \\
(\mu \mathrm{m})\end{array}$ & $\begin{array}{c}\text { Average } \\
\text { Diameter } \\
(\mu \mathrm{m})\end{array}$ & $\begin{array}{c}\text { Standard } \\
\text { Deviation } \\
(\mu \mathrm{m})\end{array}$ \\
\hline 1 & 22 & 0 & 0 & 0 & 0.00 & 0.00 \\
\hline 2 & 22 & 0 & 0 & 0 & 0.00 & 0.00 \\
\hline 3 & 24 & 0.4381 & 0.3969 & 0.491 & 0.44 & 0.04 \\
\hline 4 & 24 & 1.828 & 0.4734 & 0.3661 & 0.89 & 0.67 \\
\hline 5 & 26 & 1.056 & 0.3459 & 0.3897 & 0.60 & 0.32 \\
\hline 6 & 26 & 0.3744 & 0.3722 & 0.3027 & 0.35 & 0.03 \\
\hline 7 & 28 & 0.2992 & 0.388 & 0.3947 & 0.36 & 0.04 \\
\hline 8 & 28 & 0 & 0 & 0 & 0.00 & 0.00 \\
\hline 9 & 30 & 0.2791 & 0.4561 & 0.6034 & 0.45 & 0.13 \\
\hline 10 & 30 & 0.9771 & 0.4253 & 1.115 & 0.84 & 0.30 \\
\hline 11 & 32 & 0.5418 & 0.4986 & 1.604 & 0.88 & 0.51 \\
\hline 12 & 32 & 0 & 0 & 0 & 0.00 & 0.00 \\
\hline 13 & 34 & 0 & 0 & 0 & 0.00 & 0.00 \\
\hline 14 & 34 & 0 & 0 & 0 & 0.00 & 0.00 \\
\hline 15 & 36 & 0 & 0 & 0 & 0.00 & 0.00 \\
\hline 16 & 36 & 0 & 0 & 0 & 0.00 & 0.00 \\
\hline 17 & 38 & 0 & 0 & 0 & 0.00 & 0.00 \\
\hline 18 & 38 & 0 & 0 & 0 & 0.00 & 0.00 \\
\hline 19 & 40 & 0 & 0 & 0 & 0.00 & 0.00 \\
\hline 20 & 40 & 0 & 0 & 0 & 0.00 & 0.00 \\
\hline Yield & $40 \%$ & & & & & \\
\hline
\end{tabular}




\begin{tabular}{|c|c|c|c|c|c|c|}
\hline Concentration & $23 \%$ & & & & & \\
\hline Feed rate & $\begin{array}{c}15 \\
\mathrm{~mm} / \mathrm{s}\end{array}$ & & & & & \\
\hline Needle Gauge & 25 & & & & & \\
\hline Dispense Time & $0.02 \mathrm{~s}$ & & & & & \\
\hline \multicolumn{7}{|l|}{ Short to Long } \\
\hline Fiber \# & $\begin{array}{c}\text { Length } \\
(\mathrm{mm})\end{array}$ & $\begin{array}{c}\text { Leading } \\
\text { Diameter } \\
(\mu \mathrm{m})\end{array}$ & $\begin{array}{c}\text { Middle } \\
\text { Diameter } \\
(\mu \mathrm{m})\end{array}$ & $\begin{array}{c}\text { Terminating } \\
\text { Diameter } \\
(\mu \mathrm{m})\end{array}$ & $\begin{array}{c}\text { Average } \\
\text { Diameter } \\
(\mu \mathrm{m})\end{array}$ & $\begin{array}{c}\text { Standard } \\
\text { Deviation } \\
(\mu \mathrm{m})\end{array}$ \\
\hline 1 & 22 & 0.6598 & 0.3298 & 0.9383 & 0.64 & 0.25 \\
\hline 2 & 22 & 0.3441 & 0.2975 & 0.9542 & 0.53 & 0.30 \\
\hline 3 & 24 & 0.3663 & 0.56 & 1.183 & 0.70 & 0.35 \\
\hline 4 & 24 & 0.2135 & 0.3592 & 1.076 & 0.55 & 0.38 \\
\hline 5 & 26 & 0.3723 & 0.2835 & 1.185 & 0.61 & 0.41 \\
\hline 6 & 26 & 0 & 0 & 0 & 0.00 & 0.00 \\
\hline 7 & 28 & 0 & 0 & 0 & 0.00 & 0.00 \\
\hline 8 & 28 & 0 & 0 & 0 & 0.00 & 0.00 \\
\hline 9 & 30 & 0 & 0 & 0 & 0.00 & 0.00 \\
\hline 10 & 30 & 0 & 0 & 0 & 0.00 & 0.00 \\
\hline 11 & 32 & 0 & 0 & 0 & 0.00 & 0.00 \\
\hline 12 & 32 & 0 & 0 & 0 & 0.00 & 0.00 \\
\hline 13 & 34 & 0 & 0 & 0 & 0.00 & 0.00 \\
\hline 14 & 34 & 0 & 0 & 0 & 0.00 & 0.00 \\
\hline 15 & 36 & 0 & 0 & 0 & 0.00 & 0.00 \\
\hline 16 & 36 & 0 & 0 & 0 & 0.00 & 0.00 \\
\hline 17 & 38 & 0 & 0 & 0 & 0.00 & 0.00 \\
\hline 18 & 38 & 0 & 0 & 0 & 0.00 & 0.00 \\
\hline 19 & 40 & 0 & 0 & 0 & 0.00 & 0.00 \\
\hline 20 & 40 & 0 & 0 & 0 & 0.00 & 0.00 \\
\hline Yield & $25 \%$ & & & & & \\
\hline
\end{tabular}




\begin{tabular}{|c|c|c|c|c|c|c|}
\hline Concentration & $23 \%$ & & & & & \\
\hline Feed rate & $\begin{array}{c}22.5 \\
\mathrm{~mm} / \mathrm{s}\end{array}$ & & & & & \\
\hline Needle Gauge & 27 & & & & & \\
\hline Dispense Time & $0.02 \mathrm{~s}$ & & & & & \\
\hline \multicolumn{7}{|l|}{ Short to Long } \\
\hline Fiber \# & $\begin{array}{c}\text { Length } \\
(\mathrm{mm})\end{array}$ & $\begin{array}{c}\text { Leading } \\
\text { Diameter } \\
(\mu \mathrm{m})\end{array}$ & $\begin{array}{c}\text { Middle } \\
\text { Diameter } \\
(\mu \mathrm{m})\end{array}$ & $\begin{array}{c}\text { Terminating } \\
\text { Diameter } \\
(\mu \mathrm{m})\end{array}$ & $\begin{array}{c}\text { Average } \\
\text { Diameter } \\
(\mu \mathrm{m})\end{array}$ & $\begin{array}{c}\text { Standard } \\
\text { Deviation } \\
(\mu \mathrm{m})\end{array}$ \\
\hline 1 & 22 & 4.4 & 1.517 & 2.357 & 2.76 & 1.21 \\
\hline 2 & 22 & 0 & 0 & 0 & 0.00 & 0.00 \\
\hline 3 & 24 & 0 & 0 & 0 & 0.00 & 0.00 \\
\hline 4 & 24 & 0 & 0 & 0 & 0.00 & 0.00 \\
\hline 5 & 26 & 1.818 & 0.6097 & 0.6234 & 1.02 & 0.57 \\
\hline 6 & 26 & 1.524 & 0.5558 & 0.6295 & 0.90 & 0.44 \\
\hline 7 & 28 & 0.3563 & 0.7021 & 0.8213 & 0.63 & 0.20 \\
\hline 8 & 28 & 0.1948 & 0.1844 & 1.301 & 0.56 & 0.52 \\
\hline 9 & 30 & 0.4745 & 1.536 & 0.9328 & 0.98 & 0.43 \\
\hline 10 & 30 & 1.301 & 0.4925 & 0.740 & 0.84 & 0.34 \\
\hline 11 & 32 & 0 & 0 & 0 & 0.00 & 0.00 \\
\hline 12 & 32 & 2.388 & 0.7582 & 1.538 & 1.56 & 0.67 \\
\hline 13 & 34 & 0 & 0 & 0 & 0.00 & 0.00 \\
\hline 14 & 34 & 0.7081 & 0.1891 & 1.673 & 0.86 & 0.61 \\
\hline 15 & 36 & 0 & 0 & 0 & 0.00 & 0.00 \\
\hline 16 & 36 & 1.2 & 0.8052 & 0.5117 & 0.84 & 0.28 \\
\hline 17 & 38 & 2.926 & 0.8488 & 1.475 & 1.75 & 0.87 \\
\hline 18 & 38 & 2.533 & 1.191 & 2.428 & 2.05 & 0.61 \\
\hline 19 & 40 & 0 & 0 & 0 & 0.00 & 0.00 \\
\hline 20 & 40 & 0 & 0 & 0 & 0.00 & 0.00 \\
\hline Yield & $60 \%$ & & & & & \\
\hline
\end{tabular}




\begin{tabular}{|c|c|c|c|c|c|c|}
\hline Concentration & $23 \%$ & & & & & \\
\hline Feed rate & $\begin{array}{c}15 \\
\mathrm{~mm} / \mathrm{s}\end{array}$ & & & & & \\
\hline Needle Gauge & 27 & & & & & \\
\hline Dispense Time & $0.02 \mathrm{~s}$ & & & & & \\
\hline \multicolumn{7}{|l|}{ Short to Long } \\
\hline Fiber \# & $\begin{array}{c}\text { Length } \\
(\mathrm{mm})\end{array}$ & $\begin{array}{c}\text { Leading } \\
\text { Diameter } \\
(\mu \mathrm{m})\end{array}$ & $\begin{array}{c}\text { Middle } \\
\text { Diameter } \\
(\mu \mathrm{m})\end{array}$ & $\begin{array}{c}\text { Terminating } \\
\text { Diameter } \\
(\mu \mathrm{m})\end{array}$ & $\begin{array}{c}\text { Average } \\
\text { Diameter } \\
(\mu \mathrm{m})\end{array}$ & $\begin{array}{c}\text { Standard } \\
\text { Deviation } \\
(\mu \mathrm{m})\end{array}$ \\
\hline 1 & 22 & 1.449 & 1.070 & 1.308 & 1.28 & 0.16 \\
\hline 2 & 22 & 0.8419 & 0.7611 & 1.66 & 1.09 & 0.41 \\
\hline 3 & 24 & 0.8411 & 0.9628 & 0.758 & 0.85 & 0.08 \\
\hline 4 & 24 & 0.7707 & 0.6254 & 1.328 & 0.91 & 0.30 \\
\hline 5 & 26 & 1.443 & 0.7575 & 0.9569 & 1.05 & 0.29 \\
\hline 6 & 26 & 0.2671 & 1.667 & 0.7055 & 0.88 & 0.58 \\
\hline 7 & 28 & 0.5961 & 0.5199 & 0.9348 & 0.68 & 0.18 \\
\hline 8 & 28 & 1.847 & 1.180 & 1.492 & 1.51 & 0.27 \\
\hline 9 & 30 & 0.9494 & 0.951 & 1.195 & 1.03 & 0.12 \\
\hline 10 & 30 & 1.497 & 1.170 & 1.250 & 1.31 & 0.14 \\
\hline 11 & 32 & 1.665 & 1.004 & 1.236 & 1.30 & 0.27 \\
\hline 12 & 32 & 3.536 & 1.237 & 2.730 & 2.50 & 0.95 \\
\hline 13 & 34 & 0 & 0 & 0 & 0.00 & 0.00 \\
\hline 14 & 34 & 0 & 0 & 0 & 0.00 & 0.00 \\
\hline 15 & 36 & 0 & 0 & 0 & 0.00 & 0.00 \\
\hline 16 & 36 & 2.431 & 0.9452 & 2.115 & 1.83 & 0.64 \\
\hline 17 & 38 & 2.506 & 2.1 & 1.114 & 1.91 & 0.58 \\
\hline 18 & 38 & 0 & 0 & 0 & 0.00 & 0.00 \\
\hline 19 & 40 & 0 & 0 & 0 & 0.00 & 0.00 \\
\hline 20 & 40 & 1.474 & 1.342 & 2.476 & 1.76 & 0.51 \\
\hline Yield & $75 \%$ & & & & & \\
\hline
\end{tabular}




\begin{tabular}{|c|c|c|c|c|c|c|}
\hline Concentration & $23 \%$ & & & & & \\
\hline Feed rate & $\begin{array}{c}22.5 \\
\mathrm{~mm} / \mathrm{s}\end{array}$ & & & & & \\
\hline Needle Gauge & 30 & & & & & \\
\hline Dispense Time & $0.02 \mathrm{~s}$ & & & & & \\
\hline \multicolumn{7}{|l|}{ Short to Long } \\
\hline Fiber \# & $\begin{array}{c}\text { Length } \\
(\mathrm{mm})\end{array}$ & $\begin{array}{c}\text { Leading } \\
\text { Diameter } \\
(\mu \mathrm{m})\end{array}$ & $\begin{array}{c}\text { Middle } \\
\text { Diameter } \\
(\mu \mathrm{m})\end{array}$ & $\begin{array}{c}\text { Terminating } \\
\text { Diameter } \\
(\mu \mathrm{m})\end{array}$ & $\begin{array}{c}\text { Average } \\
\text { Diameter } \\
(\mu \mathrm{m})\end{array}$ & $\begin{array}{c}\text { Standard } \\
\text { Deviation } \\
(\mu \mathrm{m})\end{array}$ \\
\hline 1 & 22 & 0 & 0 & 0 & 0.00 & 0.00 \\
\hline 2 & 22 & 1.055 & 2.891 & 2.834 & 2.26 & 0.85 \\
\hline 3 & 24 & 0 & 0 & 0 & 0.00 & 0.00 \\
\hline 4 & 24 & 1.943 & 2.651 & 2.193 & 2.26 & 0.29 \\
\hline 5 & 26 & 1.929 & 1.996 & 0.7749 & 1.57 & 0.56 \\
\hline 6 & 26 & 0 & 0 & 0 & 0.00 & 0.00 \\
\hline 7 & 28 & 0.9949 & 1.543 & 2.313 & 1.62 & 0.54 \\
\hline 8 & 28 & 0 & 0 & 0 & 0.00 & 0.00 \\
\hline 9 & 30 & 1.379 & 0.962 & 3.066 & 1.80 & 0.91 \\
\hline 10 & 30 & 0 & 0 & 0 & 0.00 & 0.00 \\
\hline 11 & 32 & 1.681 & 2.342 & 2.811 & 2.28 & 0.46 \\
\hline 12 & 32 & 1.764 & 2.222 & 4.146 & 2.71 & 1.03 \\
\hline 13 & 34 & 0 & 0 & 0 & 0.00 & 0.00 \\
\hline 14 & 34 & 0 & 0 & 0 & 0.00 & 0.00 \\
\hline 15 & 36 & 0 & 0 & 0 & 0.00 & 0.00 \\
\hline 16 & 36 & 0 & 0 & 0 & 0.00 & 0.00 \\
\hline 17 & 38 & 0 & 0 & 0 & 0.00 & 0.00 \\
\hline 18 & 38 & 0 & 0 & 0 & 0.00 & 0.00 \\
\hline 19 & 40 & 0 & 0 & 0 & 0.00 & 0.00 \\
\hline 20 & 40 & 0 & 0 & 0 & 0.00 & 0.00 \\
\hline Yield & $35 \%$ & & & & & \\
\hline
\end{tabular}




\begin{tabular}{|c|c|c|c|c|c|c|}
\hline Concentration & $23 \%$ & & & & & \\
\hline Feed rate & $\begin{array}{c}15 \\
\mathrm{~mm} / \mathrm{s}\end{array}$ & & & & & \\
\hline Needle Gauge & 30 & & & & & \\
\hline Dispense Time & $0.02 \mathrm{~s}$ & & & & & \\
\hline \multicolumn{7}{|l|}{ Short to Long } \\
\hline Fiber \# & $\begin{array}{c}\text { Length } \\
(\mathrm{mm})\end{array}$ & $\begin{array}{c}\text { Leading } \\
\text { Diameter } \\
(\mu \mathrm{m})\end{array}$ & $\begin{array}{c}\text { Middle } \\
\text { Diameter } \\
(\mu \mathrm{m})\end{array}$ & $\begin{array}{c}\text { Terminating } \\
\text { Diameter } \\
(\mu \mathrm{m})\end{array}$ & $\begin{array}{c}\text { Average } \\
\text { Diameter } \\
(\mu \mathrm{m})\end{array}$ & $\begin{array}{c}\text { Standard } \\
\text { Deviation } \\
(\mu \mathrm{m})\end{array}$ \\
\hline 1 & 22 & 0 & 0 & 0 & 0.00 & 0.00 \\
\hline 2 & 22 & 2.811 & 2.495 & 2.828 & 2.71 & 0.15 \\
\hline 3 & 24 & 2.934 & 3.101 & 2.287 & 2.77 & 0.35 \\
\hline 4 & 24 & 3.041 & 2.707 & 3.514 & 3.09 & 0.33 \\
\hline 5 & 26 & 3.275 & 2.534 & 3.336 & 3.05 & 0.36 \\
\hline 6 & 26 & 3.740 & 2.167 & 3.062 & 2.99 & 0.64 \\
\hline 7 & 28 & 3.227 & 2.785 & 2.912 & 2.97 & 0.19 \\
\hline 8 & 28 & 3.373 & 1.749 & 2.996 & 2.71 & 0.69 \\
\hline 9 & 30 & 2.787 & 2.574 & 2.557 & 2.64 & 0.10 \\
\hline 10 & 30 & 0 & 0 & 0 & 0.00 & 0.00 \\
\hline 11 & 32 & 0 & 0 & 0 & 0.00 & 0.00 \\
\hline 12 & 32 & 0 & 0 & 0 & 0.00 & 0.00 \\
\hline 13 & 34 & 0 & 0 & 0 & 0.00 & 0.00 \\
\hline 14 & 34 & 0 & 0 & 0 & 0.00 & 0.00 \\
\hline 15 & 36 & 0 & 0 & 0 & 0.00 & 0.00 \\
\hline 16 & 36 & 0 & 0 & 0 & 0.00 & 0.00 \\
\hline 17 & 38 & 0 & 0 & 0 & 0.00 & 0.00 \\
\hline 18 & 38 & 0 & 0 & 0 & 0.00 & 0.00 \\
\hline 19 & 40 & 0 & 0 & 0 & 0.00 & 0.00 \\
\hline 20 & 40 & 0 & 0 & 0 & 0.00 & 0.00 \\
\hline Yield & $45 \%$ & & & & & \\
\hline
\end{tabular}




\begin{tabular}{|c|c|c|c|c|c|c|}
\hline Concentration & $23 \%$ & & & & & \\
\hline Feed rate & $\begin{array}{c}22.5 \\
\mathrm{~mm} / \mathrm{s}\end{array}$ & & & & & \\
\hline Needle Gauge & 32 & & & & & \\
\hline Dispense Time & $0.02 \mathrm{~s}$ & & & & & \\
\hline \multicolumn{7}{|l|}{ Short to Long } \\
\hline Fiber \# & $\begin{array}{c}\text { Length } \\
(\mathrm{mm})\end{array}$ & $\begin{array}{c}\text { Leading } \\
\text { Diameter } \\
(\mu \mathrm{m})\end{array}$ & $\begin{array}{c}\text { Middle } \\
\text { Diameter } \\
(\mu \mathrm{m})\end{array}$ & $\begin{array}{c}\text { Terminating } \\
\text { Diameter } \\
(\mu \mathrm{m})\end{array}$ & $\begin{array}{c}\text { Average } \\
\text { Diameter } \\
(\mu \mathrm{m})\end{array}$ & $\begin{array}{c}\text { Standard } \\
\text { Deviation } \\
(\mu \mathrm{m})\end{array}$ \\
\hline 1 & 22 & 0 & 0 & 0 & 0.00 & 0.00 \\
\hline 2 & 22 & 0 & 0 & 0 & 0.00 & 0.00 \\
\hline 3 & 24 & 0 & 0 & 0 & 0.00 & 0.00 \\
\hline 4 & 24 & 0 & 0 & 0 & 0.00 & 0.00 \\
\hline 5 & 26 & 2.395 & 1.248 & 1.578 & 1.74 & 0.48 \\
\hline 6 & 26 & 0 & 0 & 0 & 0.00 & 0.00 \\
\hline 7 & 28 & 0 & 0 & 0 & 0.00 & 0.00 \\
\hline 8 & 28 & 0 & 0 & 0 & 0.00 & 0.00 \\
\hline 9 & 30 & 1.079 & 1.377 & 1.769 & 1.41 & 0.28 \\
\hline 10 & 30 & 0 & 0 & 0 & 0.00 & 0.00 \\
\hline 11 & 32 & 0 & 0 & 0 & 0.00 & 0.00 \\
\hline 12 & 32 & 0.599 & 1.518 & 1.653 & 1.26 & 0.47 \\
\hline 13 & 34 & 0 & 0 & 0 & 0.00 & 0.00 \\
\hline 14 & 34 & 0 & 0 & 0 & 0.00 & 0.00 \\
\hline 15 & 36 & 0 & 0 & 0 & 0.00 & 0.00 \\
\hline 16 & 36 & 0 & 0 & 0 & 0.00 & 0.00 \\
\hline 17 & 38 & 2.205 & 1.671 & 1.696 & 1.86 & 0.25 \\
\hline 18 & 38 & 0 & 0 & 0 & 0.00 & 0.00 \\
\hline 19 & 40 & 0 & 0 & 0 & 0.00 & 0.00 \\
\hline 20 & 40 & 0 & 0 & 0 & 0.00 & 0.00 \\
\hline Yield & $20 \%$ & & & & & \\
\hline
\end{tabular}




\begin{tabular}{|c|c|c|c|c|c|c|}
\hline Concentration & $23 \%$ & & & & & \\
\hline Feed rate & $\begin{array}{c}15 \\
\mathrm{~mm} / \mathrm{s}\end{array}$ & & & & & \\
\hline Needle Gauge & 32 & & & & & \\
\hline Dispense Time & $0.02 \mathrm{~s}$ & & & & & \\
\hline \multicolumn{7}{|l|}{ Short to Long } \\
\hline Fiber \# & $\begin{array}{c}\text { Length } \\
(\mathrm{mm})\end{array}$ & $\begin{array}{c}\text { Leading } \\
\text { Diameter } \\
(\mu \mathrm{m})\end{array}$ & $\begin{array}{c}\text { Middle } \\
\text { Diameter } \\
(\mu \mathrm{m})\end{array}$ & $\begin{array}{c}\text { Terminating } \\
\text { Diameter } \\
(\mu \mathrm{m})\end{array}$ & $\begin{array}{c}\text { Average } \\
\text { Diameter } \\
(\mu \mathrm{m})\end{array}$ & $\begin{array}{c}\text { Standard } \\
\text { Deviation } \\
(\mu \mathrm{m})\end{array}$ \\
\hline 1 & 22 & 0 & 0 & 0 & 0.00 & 0.00 \\
\hline 2 & 22 & 3.162 & 2.544 & 3.473 & 3.06 & 0.39 \\
\hline 3 & 24 & 3.822 & 1.606 & 2.439 & 2.62 & 0.91 \\
\hline 4 & 24 & 0 & 0 & 0 & 0.00 & 0.00 \\
\hline 5 & 26 & 0 & 0 & 0 & 0.00 & 0.00 \\
\hline 6 & 26 & 0 & 0 & 0 & 0.00 & 0.00 \\
\hline 7 & 28 & 0 & 0 & 0 & 0.00 & 0.00 \\
\hline 8 & 28 & 0 & 0 & 0 & 0.00 & 0.00 \\
\hline 9 & 30 & 0 & 0 & 0 & 0.00 & 0.00 \\
\hline 10 & 30 & 0 & 0 & 0 & 0.00 & 0.00 \\
\hline 11 & 32 & 0 & 0 & 0 & 0.00 & 0.00 \\
\hline 12 & 32 & 0 & 0 & 0 & 0.00 & 0.00 \\
\hline 13 & 34 & 0 & 0 & 0 & 0.00 & 0.00 \\
\hline 14 & 34 & 0 & 0 & 0 & 0.00 & 0.00 \\
\hline 15 & 36 & 0 & 0 & 0 & 0.00 & 0.00 \\
\hline 16 & 36 & 0 & 0 & 0 & 0.00 & 0.00 \\
\hline 17 & 38 & 0 & 0 & 0 & 0.00 & 0.00 \\
\hline 18 & 38 & 0 & 0 & 0 & 0.00 & 0.00 \\
\hline 19 & 40 & 0 & 0 & 0 & 0.00 & 0.00 \\
\hline 20 & 40 & 0 & 0 & 0 & 0.00 & 0.00 \\
\hline Yield & $10 \%$ & & & & & \\
\hline
\end{tabular}




\begin{tabular}{|c|c|c|c|c|c|c|}
\hline Concentration & $22 \%$ & & & & & \\
\hline Feed rate & $\begin{array}{c}22.5 \\
\mathrm{~mm} / \mathrm{s}\end{array}$ & & & & & \\
\hline Needle Gauge & 25 & & & & & \\
\hline Dispense Time & $0.02 \mathrm{~s}$ & & & & & \\
\hline \multicolumn{7}{|l|}{ Short to Long } \\
\hline Fiber \# & $\begin{array}{c}\text { Length } \\
(\mathrm{mm})\end{array}$ & $\begin{array}{c}\text { Leading } \\
\text { Diameter } \\
(\mu \mathrm{m})\end{array}$ & $\begin{array}{c}\text { Middle } \\
\text { Diameter } \\
(\mu \mathrm{m})\end{array}$ & $\begin{array}{c}\text { Terminating } \\
\text { Diameter } \\
(\mu \mathrm{m})\end{array}$ & $\begin{array}{c}\text { Average } \\
\text { Diameter } \\
(\mu \mathrm{m})\end{array}$ & $\begin{array}{c}\text { Standard } \\
\text { Deviation } \\
(\mu \mathrm{m})\end{array}$ \\
\hline 1 & 22 & 0 & 0 & 0 & 0.00 & 0.00 \\
\hline 2 & 22 & 2.321 & 1.655 & 3.445 & 2.47 & 0.74 \\
\hline 3 & 24 & 1.074 & 1.261 & 3.631 & 1.99 & 1.16 \\
\hline 4 & 24 & 0.4472 & 2.149 & 2.474 & 1.69 & 0.89 \\
\hline 5 & 26 & 0.6364 & 1.979 & 0.3086 & 0.97 & 0.72 \\
\hline 6 & 26 & 1.757 & 1.893 & 3.543 & 2.40 & 0.81 \\
\hline 7 & 28 & 0.5184 & 2.648 & 3.424 & 2.20 & 1.23 \\
\hline 8 & 28 & 1.590 & 2.088 & 3.703 & 2.46 & 0.90 \\
\hline 9 & 30 & 0.7996 & 0.6476 & 1.058 & 0.84 & 0.17 \\
\hline 10 & 30 & 0.8081 & 0.6086 & 0.8354 & 0.75 & 0.10 \\
\hline 11 & 32 & 0.4479 & 0.5684 & 1.083 & 0.70 & 0.28 \\
\hline 12 & 32 & 0.9318 & 0.3641 & 0.9729 & 0.76 & 0.28 \\
\hline 13 & 34 & 0 & 0 & 0 & 0.00 & 0.00 \\
\hline 14 & 34 & 0 & 0 & 0 & 0.00 & 0.00 \\
\hline 15 & 36 & 0 & 0 & 0 & 0.00 & 0.00 \\
\hline 16 & 36 & 0 & 0 & 0 & 0.00 & 0.00 \\
\hline 17 & 38 & 0 & 0 & 0 & 0.00 & 0.00 \\
\hline 18 & 38 & 0 & 0 & 0 & 0.00 & 0.00 \\
\hline 19 & 40 & 0 & 0 & 0 & 0.00 & 0.00 \\
\hline 20 & 40 & 0 & 0 & 0 & 0.00 & 0.00 \\
\hline Yield & $55 \%$ & & & & & \\
\hline
\end{tabular}




\begin{tabular}{|c|c|c|c|c|c|c|}
\hline Concentration & $22 \%$ & & & & & \\
\hline Feed rate & $\begin{array}{c}15 \\
\mathrm{~mm} / \mathrm{s}\end{array}$ & & & & & \\
\hline Needle Gauge & 25 & & & & & \\
\hline Dispense Time & $0.02 \mathrm{~s}$ & & & & & \\
\hline \multicolumn{7}{|l|}{ Short to Long } \\
\hline Fiber \# & $\begin{array}{c}\text { Length } \\
(\mathrm{mm})\end{array}$ & $\begin{array}{c}\text { Leading } \\
\text { Diameter } \\
(\mu \mathrm{m})\end{array}$ & $\begin{array}{c}\text { Middle } \\
\text { Diameter } \\
(\mu \mathrm{m})\end{array}$ & $\begin{array}{c}\text { Terminating } \\
\text { Diameter } \\
(\mu \mathrm{m})\end{array}$ & $\begin{array}{c}\text { Average } \\
\text { Diameter } \\
(\mu \mathrm{m})\end{array}$ & $\begin{array}{c}\text { Standard } \\
\text { Deviation } \\
(\mu \mathrm{m})\end{array}$ \\
\hline 1 & 22 & 0 & 0 & 0 & 0.00 & 0.00 \\
\hline 2 & 22 & 0.6379 & 1.494 & 1.719 & 1.28 & 0.47 \\
\hline 3 & 24 & 0.6726 & 0.6925 & 0.9669 & 0.78 & 0.13 \\
\hline 4 & 24 & 1.271 & 1.718 & 1.951 & 1.65 & 0.28 \\
\hline 5 & 26 & 1.690 & 1.071 & 1.787 & 1.52 & 0.32 \\
\hline 6 & 26 & 1.115 & 1.730 & 1.428 & 1.42 & 0.25 \\
\hline 7 & 28 & 2.225 & 2.601 & 2.142 & 2.32 & 0.20 \\
\hline 8 & 28 & 0.389 & 1.122 & 2.349 & 1.29 & 0.81 \\
\hline 9 & 30 & 0 & 0 & 0 & 0.00 & 0.00 \\
\hline 10 & 30 & 0 & 0 & 0 & 0.00 & 0.00 \\
\hline 11 & 32 & 0 & 0 & 0 & 0.00 & 0.00 \\
\hline 12 & 32 & 0 & 0 & 0 & 0.00 & 0.00 \\
\hline 13 & 34 & 0 & 0 & 0 & 0.00 & 0.00 \\
\hline 14 & 34 & 1.353 & 1.9 & 1.137 & 1.46 & 0.32 \\
\hline 15 & 36 & 0 & 0 & 0 & 0.00 & 0.00 \\
\hline 16 & 36 & 0 & 0 & 0 & 0.00 & 0.00 \\
\hline 17 & 38 & 0 & 0 & 0 & 0.00 & 0.00 \\
\hline 18 & 38 & 0 & 0 & 0 & 0.00 & 0.00 \\
\hline 19 & 40 & 0 & 0 & 0 & 0.00 & 0.00 \\
\hline 20 & 40 & 0 & 0 & 0 & 0.00 & 0.00 \\
\hline Yield & $40 \%$ & & & & & \\
\hline
\end{tabular}




\begin{tabular}{|c|c|c|c|c|c|c|}
\hline Concentration & $22 \%$ & & & & & \\
\hline Feed rate & $\begin{array}{c}22.5 \\
\mathrm{~mm} / \mathrm{s}\end{array}$ & & & & & \\
\hline Needle Gauge & 27 & & & & & \\
\hline Dispense Time & $0.02 \mathrm{~s}$ & & & & & \\
\hline \multicolumn{7}{|l|}{ Short to Long } \\
\hline Fiber \# & $\begin{array}{c}\text { Length } \\
(\mathrm{mm})\end{array}$ & $\begin{array}{c}\text { Leading } \\
\text { Diameter } \\
(\mu \mathrm{m})\end{array}$ & $\begin{array}{c}\text { Middle } \\
\text { Diameter } \\
(\mu \mathrm{m})\end{array}$ & $\begin{array}{c}\text { Terminating } \\
\text { Diameter } \\
(\mu \mathrm{m})\end{array}$ & $\begin{array}{c}\text { Average } \\
\text { Diameter } \\
(\mu \mathrm{m})\end{array}$ & $\begin{array}{c}\text { Standard } \\
\text { Deviation } \\
(\mu \mathrm{m})\end{array}$ \\
\hline 1 & 22 & 0 & 0 & 0 & 0.00 & 0.00 \\
\hline 2 & 22 & 0.988 & 2.317 & 1.196 & 1.50 & 0.58 \\
\hline 3 & 24 & 0.9235 & 1.698 & 2.125 & 1.58 & 0.50 \\
\hline 4 & 24 & 1.087 & 1.791 & 1.881 & 1.59 & 0.35 \\
\hline 5 & 26 & 0.9255 & 3.856 & 1.434 & 2.07 & 1.28 \\
\hline 6 & 26 & 0.7756 & 1.440 & 1.471 & 1.23 & 0.32 \\
\hline 7 & 28 & 1.120 & 1.428 & 1.033 & 1.19 & 0.17 \\
\hline 8 & 28 & 1.149 & 3.621 & 0.8619 & 1.88 & 1.24 \\
\hline 9 & 30 & 1.882 & 4.617 & 1.267 & 2.59 & 1.46 \\
\hline 10 & 30 & 1.247 & 3.858 & 0.931 & 2.01 & 1.31 \\
\hline 11 & 32 & 1.512 & 1.316 & 0.8848 & 1.24 & 0.26 \\
\hline 12 & 32 & 1.155 & 3.635 & 0.9239 & 1.90 & 1.23 \\
\hline 13 & 34 & 0.9646 & 1.555 & 1.349 & 1.29 & 0.24 \\
\hline 14 & 34 & 0.9649 & 1.461 & 1.570 & 1.33 & 0.26 \\
\hline 15 & 36 & 0.8571 & 1.152 & 1.243 & 1.08 & 0.16 \\
\hline 16 & 36 & 0.8767 & 3.873 & 0.6741 & 1.81 & 1.46 \\
\hline 17 & 38 & 0.7946 & 1.452 & 1.228 & 1.16 & 0.27 \\
\hline 18 & 38 & 1.183 & 1.313 & 2.164 & 1.55 & 0.44 \\
\hline 19 & 40 & 1.676 & 2.393 & 0.9895 & 1.69 & 0.57 \\
\hline 20 & 40 & 0 & 0 & 0 & 0.00 & 0.00 \\
\hline Yield & $90 \%$ & & & & & \\
\hline
\end{tabular}




\begin{tabular}{|c|c|c|c|c|c|c|}
\hline Concentration & $22 \%$ & & & & & \\
\hline Feed rate & $\begin{array}{c}15 \\
\mathrm{~mm} / \mathrm{s}\end{array}$ & & & & & \\
\hline Needle Gauge & 27 & & & & & \\
\hline Dispense Time & $0.02 \mathrm{~s}$ & & & & & \\
\hline \multicolumn{7}{|l|}{ Short to Long } \\
\hline Fiber \# & $\begin{array}{c}\text { Length } \\
(\mathrm{mm})\end{array}$ & $\begin{array}{c}\text { Leading } \\
\text { Diameter } \\
(\mu \mathrm{m})\end{array}$ & $\begin{array}{c}\text { Middle } \\
\text { Diameter } \\
(\mu \mathrm{m})\end{array}$ & $\begin{array}{c}\text { Terminating } \\
\text { Diameter } \\
(\mu \mathrm{m})\end{array}$ & $\begin{array}{c}\text { Average } \\
\text { Diameter } \\
(\mu \mathrm{m})\end{array}$ & $\begin{array}{c}\text { Standard } \\
\text { Deviation } \\
(\mu \mathrm{m})\end{array}$ \\
\hline 1 & 22 & 0 & 0 & 0 & 0.00 & 0.00 \\
\hline 2 & 22 & 1.197 & 4.823 & 1.989 & 2.67 & 1.56 \\
\hline 3 & 24 & 3.295 & 1.355 & 0.5373 & 1.73 & 1.16 \\
\hline 4 & 24 & 0 & 0 & 0 & 0.00 & 0.00 \\
\hline 5 & 26 & 0 & 0 & 0 & 0.00 & 0.00 \\
\hline 6 & 26 & 0.5227 & 1.017 & 3.7 & 1.75 & 1.40 \\
\hline 7 & 28 & 0.5582 & 0.5543 & 2.792 & 1.30 & 1.05 \\
\hline 8 & 28 & 1.301 & 1.098 & 0.5457 & 0.98 & 0.32 \\
\hline 9 & 30 & 0 & 0 & 0 & 0.00 & 0.00 \\
\hline 10 & 30 & 0 & 0 & 0 & 0.00 & 0.00 \\
\hline 11 & 32 & 0 & 0 & 0 & 0.00 & 0.00 \\
\hline 12 & 32 & 0 & 0 & 0 & 0.00 & 0.00 \\
\hline 13 & 34 & 0 & 0 & 0 & 0.00 & 0.00 \\
\hline 14 & 34 & 0 & 0 & 0 & 0.00 & 0.00 \\
\hline 15 & 36 & 0 & 0 & 0 & 0.00 & 0.00 \\
\hline 16 & 36 & 0 & 0 & 0 & 0.00 & 0.00 \\
\hline 17 & 38 & 0 & 0 & 0 & 0.00 & 0.00 \\
\hline 18 & 38 & 0 & 0 & 0 & 0.00 & 0.00 \\
\hline 19 & 40 & 0 & 0 & 0 & 0.00 & 0.00 \\
\hline 20 & 40 & 0 & 0 & 0 & 0.00 & 0.00 \\
\hline Yield & $25 \%$ & & & & & \\
\hline
\end{tabular}




\begin{tabular}{|c|c|c|c|c|c|c|}
\hline Concentration & $22 \%$ & & & & & \\
\hline Feed rate & $\begin{array}{c}22.5 \\
\mathrm{~mm} / \mathrm{s}\end{array}$ & & & & & \\
\hline Needle Gauge & 30 & & & & & \\
\hline Dispense Time & $0.02 \mathrm{~s}$ & & & & & \\
\hline \multicolumn{7}{|l|}{ Short to Long } \\
\hline Fiber \# & $\begin{array}{c}\text { Length } \\
(\mathrm{mm})\end{array}$ & $\begin{array}{c}\text { Leading } \\
\text { Diameter } \\
(\mu \mathrm{m})\end{array}$ & $\begin{array}{c}\text { Middle } \\
\text { Diameter } \\
(\mu \mathrm{m})\end{array}$ & $\begin{array}{c}\text { Terminating } \\
\text { Diameter } \\
(\mu \mathrm{m})\end{array}$ & $\begin{array}{c}\text { Average } \\
\text { Diameter } \\
(\mu \mathrm{m})\end{array}$ & $\begin{array}{c}\text { Standard } \\
\text { Deviation } \\
(\mu \mathrm{m})\end{array}$ \\
\hline 1 & 22 & 0 & 0 & 0 & 0.00 & 0.00 \\
\hline 2 & 22 & 4.529 & 0.9043 & 8.916 & 4.78 & 3.28 \\
\hline 3 & 24 & 5.168 & 5.054 & 8.563 & 6.26 & 1.63 \\
\hline 4 & 24 & 8.894 & 7.774 & 4.627 & 7.10 & 1.81 \\
\hline 5 & 26 & 4.040 & 7.251 & 5.229 & 5.51 & 1.33 \\
\hline 6 & 26 & 5.474 & 4.897 & 8.323 & 6.23 & 1.50 \\
\hline 7 & 28 & 3.240 & 3.112 & 2.501 & 2.95 & 0.32 \\
\hline 8 & 28 & 1.649 & 3.520 & 4.531 & 3.23 & 1.19 \\
\hline 9 & 30 & 2.724 & 2.442 & 2.345 & 2.50 & 0.16 \\
\hline 10 & 30 & 0 & 0 & 0 & 0.00 & 0.00 \\
\hline 11 & 32 & 0 & 0 & 0 & 0.00 & 0.00 \\
\hline 12 & 32 & 0 & 0 & 0 & 0.00 & 0.00 \\
\hline 13 & 34 & 0 & 0 & 0 & 0.00 & 0.00 \\
\hline 14 & 34 & 3.642 & 2.783 & 3.388 & 3.27 & 0.36 \\
\hline 15 & 36 & 0 & 0 & 0 & 0.00 & 0.00 \\
\hline 16 & 36 & 0 & 0 & 0 & 0.00 & 0.00 \\
\hline 17 & 38 & 3.701 & 4.396 & 4.423 & 4.17 & 0.33 \\
\hline 18 & 38 & 0 & 0 & 0 & 0.00 & 0.00 \\
\hline 19 & 40 & 0 & 0 & 0 & 0.00 & 0.00 \\
\hline 20 & 40 & 0 & 0 & 0 & 0.00 & 0.00 \\
\hline Yield & $50 \%$ & & & & & \\
\hline
\end{tabular}




\begin{tabular}{|c|c|c|c|c|c|c|}
\hline Concentration & $22 \%$ & & & & & \\
\hline Feed rate & $\begin{array}{c}15 \\
\mathrm{~mm} / \mathrm{s}\end{array}$ & & & & & \\
\hline Needle Gauge & 30 & & & & & \\
\hline Dispense Time & $0.02 \mathrm{~s}$ & & & & & \\
\hline \multicolumn{7}{|l|}{ Short to Long } \\
\hline Fiber \# & $\begin{array}{c}\text { Length } \\
(\mathrm{mm})\end{array}$ & $\begin{array}{c}\text { Leading } \\
\text { Diameter } \\
(\mu \mathrm{m})\end{array}$ & $\begin{array}{c}\text { Middle } \\
\text { Diameter } \\
(\mu \mathrm{m})\end{array}$ & $\begin{array}{c}\text { Terminating } \\
\text { Diameter } \\
(\mu \mathrm{m})\end{array}$ & $\begin{array}{c}\text { Average } \\
\text { Diameter } \\
(\mu \mathrm{m})\end{array}$ & $\begin{array}{c}\text { Standard } \\
\text { Deviation } \\
(\mu \mathrm{m})\end{array}$ \\
\hline 1 & 22 & 0 & 0 & 0 & 0.00 & 0.00 \\
\hline 2 & 22 & 4.763 & 8.021 & 7.611 & 6.80 & 1.45 \\
\hline 3 & 24 & 0 & 0 & 0 & 0.00 & 0.00 \\
\hline 4 & 24 & 0 & 0 & 0 & 0.00 & 0.00 \\
\hline 5 & 26 & 0 & 0 & 0 & 0.00 & 0.00 \\
\hline 6 & 26 & 0 & 0 & 0 & 0.00 & 0.00 \\
\hline 7 & 28 & 8.016 & 5.772 & 10.46 & 8.08 & 1.91 \\
\hline 8 & 28 & 0 & 0 & 0 & 0.00 & 0.00 \\
\hline 9 & 30 & 0 & 0 & 0 & 0.00 & 0.00 \\
\hline 10 & 30 & 0 & 0 & 0 & 0.00 & 0.00 \\
\hline 11 & 32 & 0 & 0 & 0 & 0.00 & 0.00 \\
\hline 12 & 32 & 0 & 0 & 0 & 0.00 & 0.00 \\
\hline 13 & 34 & 0 & 0 & 0 & 0.00 & 0.00 \\
\hline 14 & 34 & 0 & 0 & 0 & 0.00 & 0.00 \\
\hline 15 & 36 & 0 & 0 & 0 & 0.00 & 0.00 \\
\hline 16 & 36 & 0 & 0 & 0 & 0.00 & 0.00 \\
\hline 17 & 38 & 0 & 0 & 0 & 0.00 & 0.00 \\
\hline 18 & 38 & 0 & 0 & 0 & 0.00 & 0.00 \\
\hline 19 & 40 & 0 & 0 & 0 & 0.00 & 0.00 \\
\hline 20 & 40 & 0 & 0 & 0 & 0.00 & 0.00 \\
\hline Yield & $10 \%$ & & & & & \\
\hline
\end{tabular}




\begin{tabular}{|c|c|c|c|c|c|c|}
\hline Concentration & $22 \%$ & & & & & \\
\hline Feed rate & $\begin{array}{c}22.5 \\
\mathrm{~mm} / \mathrm{s}\end{array}$ & & & & & \\
\hline Needle Gauge & 32 & & & & & \\
\hline Dispense Time & $0.02 \mathrm{~s}$ & & & & & \\
\hline \multicolumn{7}{|l|}{ Short to Long } \\
\hline Fiber \# & $\begin{array}{c}\text { Length } \\
(\mathrm{mm})\end{array}$ & $\begin{array}{c}\text { Leading } \\
\text { Diameter } \\
(\mu \mathrm{m})\end{array}$ & $\begin{array}{c}\text { Middle } \\
\text { Diameter } \\
(\mu \mathrm{m})\end{array}$ & $\begin{array}{c}\text { Terminating } \\
\text { Diameter } \\
(\mu \mathrm{m})\end{array}$ & $\begin{array}{c}\text { Average } \\
\text { Diameter } \\
(\mu \mathrm{m})\end{array}$ & $\begin{array}{c}\text { Standard } \\
\text { Deviation } \\
(\mu \mathrm{m})\end{array}$ \\
\hline 1 & 22 & 0 & 0 & 0 & & \\
\hline 2 & 22 & 0 & 0 & 0 & & \\
\hline 3 & 24 & 0 & 0 & 0 & & \\
\hline 4 & 24 & 0 & 0 & 0 & & \\
\hline 5 & 26 & 0 & 0 & 0 & & \\
\hline 6 & 26 & 0 & 0 & 0 & & \\
\hline 7 & 28 & 0 & 0 & 0 & & \\
\hline 8 & 28 & 0 & 0 & 0 & & \\
\hline 9 & 30 & 0 & 0 & 0 & & \\
\hline 10 & 30 & 0 & 0 & 0 & & \\
\hline 11 & 32 & 0 & 0 & 0 & & \\
\hline 12 & 32 & 0 & 0 & 0 & & \\
\hline 13 & 34 & 0 & 0 & 0 & & \\
\hline 14 & 34 & 0 & 0 & 0 & & \\
\hline 15 & 36 & 0 & 0 & 0 & & \\
\hline 16 & 36 & 0 & 0 & 0 & & \\
\hline 17 & 38 & 0 & 0 & 0 & & \\
\hline 18 & 38 & 0 & 0 & 0 & & \\
\hline 19 & 40 & 0 & 0 & 0 & & \\
\hline 20 & 40 & 0 & 0 & 0 & & \\
\hline Yield & $0 \%$ & & & & & \\
\hline
\end{tabular}




\begin{tabular}{|c|c|c|c|c|c|c|}
\hline Concentration & $22 \%$ & & & & & \\
\hline Feed rate & $\begin{array}{c}15 \\
\mathrm{~mm} / \mathrm{s}\end{array}$ & & & & & \\
\hline Needle Gauge & 32 & & & & & \\
\hline Dispense Time & $0.02 \mathrm{~s}$ & & & & & \\
\hline \multicolumn{7}{|l|}{ Short to Long } \\
\hline Fiber \# & $\begin{array}{c}\text { Length } \\
(\mathrm{mm})\end{array}$ & $\begin{array}{l}\text { Leading } \\
\text { Diameter } \\
(\mu \mathrm{m})\end{array}$ & $\begin{array}{c}\text { Middle } \\
\text { Diameter } \\
(\mu \mathrm{m})\end{array}$ & $\begin{array}{l}\text { Terminating } \\
\text { Diameter } \\
(\mu \mathrm{m})\end{array}$ & $\begin{array}{c}\text { Average } \\
\text { Diameter } \\
(\mu \mathrm{m})\end{array}$ & $\begin{array}{c}\text { Standard } \\
\text { Deviation } \\
(\mu \mathrm{m})\end{array}$ \\
\hline 1 & 22 & 0 & 0 & 0 & & \\
\hline 2 & 22 & 0 & 0 & 0 & & \\
\hline 3 & 24 & 0 & 0 & 0 & & \\
\hline 4 & 24 & 0 & 0 & 0 & & \\
\hline 5 & 26 & 0 & 0 & 0 & & \\
\hline 6 & 26 & 0 & 0 & 0 & & \\
\hline 7 & 28 & 0 & 0 & 0 & & \\
\hline 8 & 28 & 0 & 0 & 0 & & \\
\hline 9 & 30 & 0 & 0 & 0 & & \\
\hline 10 & 30 & 0 & 0 & 0 & & \\
\hline 11 & 32 & 0 & 0 & 0 & & \\
\hline 12 & 32 & 0 & 0 & 0 & & \\
\hline 13 & 34 & 0 & 0 & 0 & & \\
\hline 14 & 34 & 0 & 0 & 0 & & \\
\hline 15 & 36 & 0 & 0 & 0 & & \\
\hline 16 & 36 & 0 & 0 & 0 & & \\
\hline 17 & 38 & 0 & 0 & 0 & & \\
\hline 18 & 38 & 0 & 0 & 0 & & \\
\hline 19 & 40 & 0 & 0 & 0 & & \\
\hline 20 & 40 & 0 & 0 & 0 & & \\
\hline Yield & $0 \%$ & & & & & \\
\hline
\end{tabular}




\begin{tabular}{|c|c|c|c|c|c|c|}
\hline Concentration & $21 \%$ & & & & & \\
\hline Feed rate & $\begin{array}{c}22.5 \\
\mathrm{~mm} / \mathrm{s}\end{array}$ & & & & & \\
\hline Needle Gauge & 25 & & & & & \\
\hline Dispense Time & $0.02 \mathrm{~s}$ & & & & & \\
\hline \multicolumn{7}{|l|}{ Short to Long } \\
\hline Fiber \# & $\begin{array}{c}\text { Length } \\
(\mathrm{mm})\end{array}$ & $\begin{array}{l}\text { Leading } \\
\text { Diameter } \\
(\mu \mathrm{m})\end{array}$ & $\begin{array}{c}\text { Middle } \\
\text { Diameter } \\
(\mu \mathrm{m})\end{array}$ & $\begin{array}{l}\text { Terminating } \\
\text { Diameter } \\
(\mu \mathrm{m})\end{array}$ & $\begin{array}{c}\text { Average } \\
\text { Diameter } \\
(\mu \mathrm{m})\end{array}$ & $\begin{array}{c}\text { Standard } \\
\text { Deviation } \\
(\mu \mathrm{m})\end{array}$ \\
\hline 1 & 22 & 0 & 0 & 0 & & \\
\hline 2 & 22 & 0 & 0 & 0 & & \\
\hline 3 & 24 & 0 & 0 & 0 & & \\
\hline 4 & 24 & 0 & 0 & 0 & & \\
\hline 5 & 26 & 0 & 0 & 0 & & \\
\hline 6 & 26 & 0 & 0 & 0 & & \\
\hline 7 & 28 & 0 & 0 & 0 & & \\
\hline 8 & 28 & 0 & 0 & 0 & & \\
\hline 9 & 30 & 0 & 0 & 0 & & \\
\hline 10 & 30 & 0 & 0 & 0 & & \\
\hline 11 & 32 & 0 & 0 & 0 & & \\
\hline 12 & 32 & 0 & 0 & 0 & & \\
\hline 13 & 34 & 0 & 0 & 0 & & \\
\hline 14 & 34 & 0 & 0 & 0 & & \\
\hline 15 & 36 & 0 & 0 & 0 & & \\
\hline 16 & 36 & 0 & 0 & 0 & & \\
\hline 17 & 38 & 0 & 0 & 0 & & \\
\hline 18 & 38 & 0 & 0 & 0 & & \\
\hline 19 & 40 & 0 & 0 & 0 & & \\
\hline 20 & 40 & 0 & 0 & 0 & & \\
\hline Yield & $0 \%$ & & & & & \\
\hline
\end{tabular}




\begin{tabular}{|c|c|c|c|c|c|c|}
\hline Concentration & $21 \%$ & & & & & \\
\hline Feed rate & $\begin{array}{c}15 \\
\mathrm{~mm} / \mathrm{s}\end{array}$ & & & & & \\
\hline Needle Gauge & 25 & & & & & \\
\hline Dispense Time & $0.02 \mathrm{~s}$ & & & & & \\
\hline \multicolumn{7}{|l|}{ Short to Long } \\
\hline Fiber \# & $\begin{array}{c}\text { Length } \\
(\mathrm{mm})\end{array}$ & $\begin{array}{c}\text { Leading } \\
\text { Diameter } \\
(\mu \mathrm{m})\end{array}$ & $\begin{array}{c}\text { Middle } \\
\text { Diameter } \\
(\mu \mathrm{m})\end{array}$ & $\begin{array}{c}\text { Terminating } \\
\text { Diameter } \\
(\mu \mathrm{m})\end{array}$ & $\begin{array}{c}\text { Average } \\
\text { Diameter } \\
(\mu \mathrm{m})\end{array}$ & $\begin{array}{c}\text { Standard } \\
\text { Deviation } \\
(\mu \mathrm{m})\end{array}$ \\
\hline 1 & 22 & 0 & 0 & 0 & & \\
\hline 2 & 22 & 0 & 0 & 0 & & \\
\hline 3 & 24 & 0 & 0 & 0 & & \\
\hline 4 & 24 & 0 & 0 & 0 & & \\
\hline 5 & 26 & 0 & 0 & 0 & & \\
\hline 6 & 26 & 0 & 0 & 0 & & \\
\hline 7 & 28 & 0 & 0 & 0 & & \\
\hline 8 & 28 & 0 & 0 & 0 & & \\
\hline 9 & 30 & 0 & 0 & 0 & & \\
\hline 10 & 30 & 0 & 0 & 0 & & \\
\hline 11 & 32 & 0 & 0 & 0 & & \\
\hline 12 & 32 & 0 & 0 & 0 & & \\
\hline 13 & 34 & 0 & 0 & 0 & & \\
\hline 14 & 34 & 0 & 0 & 0 & & \\
\hline 15 & 36 & 0 & 0 & 0 & & \\
\hline 16 & 36 & 0 & 0 & 0 & & \\
\hline 17 & 38 & 0 & 0 & 0 & & \\
\hline 18 & 38 & 0 & 0 & 0 & & \\
\hline 19 & 40 & 0 & 0 & 0 & & \\
\hline 20 & 40 & 0 & 0 & 0 & & \\
\hline Yield & $0 \%$ & & & & & \\
\hline
\end{tabular}




\begin{tabular}{|c|c|c|c|c|c|c|}
\hline Concentration & $21 \%$ & & & & & \\
\hline Feed rate & $\begin{array}{c}22.5 \\
\mathrm{~mm} / \mathrm{s}\end{array}$ & & & & & \\
\hline Needle Gauge & 27 & & & & & \\
\hline Dispense Time & $0.02 \mathrm{~s}$ & & & & & \\
\hline \multicolumn{7}{|l|}{ Short to Long } \\
\hline Fiber \# & $\begin{array}{c}\text { Length } \\
(\mathrm{mm})\end{array}$ & $\begin{array}{l}\text { Leading } \\
\text { Diameter } \\
(\mu \mathrm{m})\end{array}$ & $\begin{array}{c}\text { Middle } \\
\text { Diameter } \\
(\mu \mathrm{m})\end{array}$ & $\begin{array}{l}\text { Terminating } \\
\text { Diameter } \\
(\mu \mathrm{m})\end{array}$ & $\begin{array}{c}\text { Average } \\
\text { Diameter } \\
(\mu \mathrm{m})\end{array}$ & $\begin{array}{c}\text { Standard } \\
\text { Deviation } \\
(\mu \mathrm{m})\end{array}$ \\
\hline 1 & 22 & 0 & 0 & 0 & & \\
\hline 2 & 22 & 0 & 0 & 0 & & \\
\hline 3 & 24 & 0 & 0 & 0 & & \\
\hline 4 & 24 & 0 & 0 & 0 & & \\
\hline 5 & 26 & 0 & 0 & 0 & & \\
\hline 6 & 26 & 0 & 0 & 0 & & \\
\hline 7 & 28 & 0 & 0 & 0 & & \\
\hline 8 & 28 & 0 & 0 & 0 & & \\
\hline 9 & 30 & 0 & 0 & 0 & & \\
\hline 10 & 30 & 0 & 0 & 0 & & \\
\hline 11 & 32 & 0 & 0 & 0 & & \\
\hline 12 & 32 & 0 & 0 & 0 & & \\
\hline 13 & 34 & 0 & 0 & 0 & & \\
\hline 14 & 34 & 0 & 0 & 0 & & \\
\hline 15 & 36 & 0 & 0 & 0 & & \\
\hline 16 & 36 & 0 & 0 & 0 & & \\
\hline 17 & 38 & 0 & 0 & 0 & & \\
\hline 18 & 38 & 0 & 0 & 0 & & \\
\hline 19 & 40 & 0 & 0 & 0 & & \\
\hline 20 & 40 & 0 & 0 & 0 & & \\
\hline Yield & $0 \%$ & & & & & \\
\hline
\end{tabular}




\begin{tabular}{|c|c|c|c|c|c|c|}
\hline Concentration & $21 \%$ & & & & & \\
\hline Feed rate & $\begin{array}{c}15 \\
\mathrm{~mm} / \mathrm{s}\end{array}$ & & & & & \\
\hline Needle Gauge & 27 & & & & & \\
\hline Dispense Time & $0.02 \mathrm{~s}$ & & & & & \\
\hline \multicolumn{7}{|l|}{ Short to Long } \\
\hline Fiber \# & $\begin{array}{c}\text { Length } \\
(\mathrm{mm})\end{array}$ & $\begin{array}{c}\text { Leading } \\
\text { Diameter } \\
(\mu \mathrm{m})\end{array}$ & $\begin{array}{c}\text { Middle } \\
\text { Diameter } \\
(\mu \mathrm{m})\end{array}$ & $\begin{array}{c}\text { Terminating } \\
\text { Diameter } \\
(\mu \mathrm{m})\end{array}$ & $\begin{array}{c}\text { Average } \\
\text { Diameter } \\
(\mu \mathrm{m})\end{array}$ & $\begin{array}{c}\text { Standard } \\
\text { Deviation } \\
(\mu \mathrm{m})\end{array}$ \\
\hline 1 & 22 & 0 & 0 & 0 & & \\
\hline 2 & 22 & 0 & 0 & 0 & & \\
\hline 3 & 24 & 0 & 0 & 0 & & \\
\hline 4 & 24 & 0 & 0 & 0 & & \\
\hline 5 & 26 & 0 & 0 & 0 & & \\
\hline 6 & 26 & 0 & 0 & 0 & & \\
\hline 7 & 28 & 0 & 0 & 0 & & \\
\hline 8 & 28 & 0 & 0 & 0 & & \\
\hline 9 & 30 & 0 & 0 & 0 & & \\
\hline 10 & 30 & 0 & 0 & 0 & & \\
\hline 11 & 32 & 0 & 0 & 0 & & \\
\hline 12 & 32 & 0 & 0 & 0 & & \\
\hline 13 & 34 & 0 & 0 & 0 & & \\
\hline 14 & 34 & 0 & 0 & 0 & & \\
\hline 15 & 36 & 0 & 0 & 0 & & \\
\hline 16 & 36 & 0 & 0 & 0 & & \\
\hline 17 & 38 & 0 & 0 & 0 & & \\
\hline 18 & 38 & 0 & 0 & 0 & & \\
\hline 19 & 40 & 0 & 0 & 0 & & \\
\hline 20 & 40 & 0 & 0 & 0 & & \\
\hline Yield & $0 \%$ & & & & & \\
\hline
\end{tabular}




\begin{tabular}{|c|c|c|c|c|c|c|}
\hline Concentration & $21 \%$ & & & & & \\
\hline Feed rate & $\begin{array}{c}22.5 \\
\mathrm{~mm} / \mathrm{s}\end{array}$ & & & & & \\
\hline Needle Gauge & 30 & & & & & \\
\hline Dispense Time & $0.02 \mathrm{~s}$ & & & & & \\
\hline \multicolumn{7}{|l|}{ Short to Long } \\
\hline Fiber \# & $\begin{array}{c}\text { Length } \\
(\mathrm{mm})\end{array}$ & $\begin{array}{c}\text { Leading } \\
\text { Diameter } \\
(\mu \mathrm{m})\end{array}$ & $\begin{array}{c}\text { Middle } \\
\text { Diameter } \\
(\mu \mathrm{m})\end{array}$ & $\begin{array}{c}\text { Terminating } \\
\text { Diameter } \\
(\mu \mathrm{m})\end{array}$ & $\begin{array}{c}\text { Average } \\
\text { Diameter } \\
(\mu \mathrm{m})\end{array}$ & $\begin{array}{c}\text { Standard } \\
\text { Deviation } \\
(\mu \mathrm{m})\end{array}$ \\
\hline 1 & 22 & 0 & 0 & 0 & & \\
\hline 2 & 22 & 0 & 0 & 0 & & \\
\hline 3 & 24 & 0 & 0 & 0 & & \\
\hline 4 & 24 & 0 & 0 & 0 & & \\
\hline 5 & 26 & 0 & 0 & 0 & & \\
\hline 6 & 26 & 0 & 0 & 0 & & \\
\hline 7 & 28 & 0 & 0 & 0 & & \\
\hline 8 & 28 & 0 & 0 & 0 & & \\
\hline 9 & 30 & 0 & 0 & 0 & & \\
\hline 10 & 30 & 0 & 0 & 0 & & \\
\hline 11 & 32 & 0 & 0 & 0 & & \\
\hline 12 & 32 & 0 & 0 & 0 & & \\
\hline 13 & 34 & 0 & 0 & 0 & & \\
\hline 14 & 34 & 0 & 0 & 0 & & \\
\hline 15 & 36 & 0 & 0 & 0 & & \\
\hline 16 & 36 & 0 & 0 & 0 & & \\
\hline 17 & 38 & 0 & 0 & 0 & & \\
\hline 18 & 38 & 0 & 0 & 0 & & \\
\hline 19 & 40 & 0 & 0 & 0 & & \\
\hline 20 & 40 & 0 & 0 & 0 & & \\
\hline Yield & $0 \%$ & & & & & \\
\hline
\end{tabular}




\begin{tabular}{|c|c|c|c|c|c|c|}
\hline Concentration & $21 \%$ & & & & & \\
\hline Feed rate & $\begin{array}{c}15 \\
\mathrm{~mm} / \mathrm{s}\end{array}$ & & & & & \\
\hline Needle Gauge & 30 & & & & & \\
\hline Dispense Time & $0.02 \mathrm{~s}$ & & & & & \\
\hline \multicolumn{7}{|l|}{ Short to Long } \\
\hline Fiber \# & $\begin{array}{c}\text { Length } \\
(\mathrm{mm})\end{array}$ & $\begin{array}{c}\text { Leading } \\
\text { Diameter } \\
(\mu \mathrm{m})\end{array}$ & $\begin{array}{c}\text { Middle } \\
\text { Diameter } \\
(\mu \mathrm{m})\end{array}$ & $\begin{array}{c}\text { Terminating } \\
\text { Diameter } \\
(\mu \mathrm{m})\end{array}$ & $\begin{array}{c}\text { Average } \\
\text { Diameter } \\
(\mu \mathrm{m})\end{array}$ & $\begin{array}{c}\text { Standard } \\
\text { Deviation } \\
(\mu \mathrm{m})\end{array}$ \\
\hline 1 & 22 & 0 & 0 & 0 & & \\
\hline 2 & 22 & 0 & 0 & 0 & & \\
\hline 3 & 24 & 0 & 0 & 0 & & \\
\hline 4 & 24 & 0 & 0 & 0 & & \\
\hline 5 & 26 & 0 & 0 & 0 & & \\
\hline 6 & 26 & 0 & 0 & 0 & & \\
\hline 7 & 28 & 0 & 0 & 0 & & \\
\hline 8 & 28 & 0 & 0 & 0 & & \\
\hline 9 & 30 & 0 & 0 & 0 & & \\
\hline 10 & 30 & 0 & 0 & 0 & & \\
\hline 11 & 32 & 0 & 0 & 0 & & \\
\hline 12 & 32 & 0 & 0 & 0 & & \\
\hline 13 & 34 & 0 & 0 & 0 & & \\
\hline 14 & 34 & 0 & 0 & 0 & & \\
\hline 15 & 36 & 0 & 0 & 0 & & \\
\hline 16 & 36 & 0 & 0 & 0 & & \\
\hline 17 & 38 & 0 & 0 & 0 & & \\
\hline 18 & 38 & 0 & 0 & 0 & & \\
\hline 19 & 40 & 0 & 0 & 0 & & \\
\hline 20 & 40 & 0 & 0 & 0 & & \\
\hline Yield & $0 \%$ & & & & & \\
\hline
\end{tabular}




\begin{tabular}{|c|c|c|c|c|c|c|}
\hline Concentration & $21 \%$ & & & & & \\
\hline Feed rate & $\begin{array}{c}22.5 \\
\mathrm{~mm} / \mathrm{s}\end{array}$ & & & & & \\
\hline Needle Gauge & 32 & & & & & \\
\hline Dispense Time & $0.02 \mathrm{~s}$ & & & & & \\
\hline \multicolumn{7}{|l|}{ Short to Long } \\
\hline Fiber \# & $\begin{array}{c}\text { Length } \\
(\mathrm{mm})\end{array}$ & $\begin{array}{c}\text { Leading } \\
\text { Diameter } \\
(\mu \mathrm{m})\end{array}$ & $\begin{array}{c}\text { Middle } \\
\text { Diameter } \\
(\mu \mathrm{m})\end{array}$ & $\begin{array}{c}\text { Terminating } \\
\text { Diameter } \\
(\mu \mathrm{m})\end{array}$ & $\begin{array}{c}\text { Average } \\
\text { Diameter } \\
(\mu \mathrm{m})\end{array}$ & $\begin{array}{c}\text { Standard } \\
\text { Deviation } \\
(\mu \mathrm{m})\end{array}$ \\
\hline 1 & 22 & 0 & 0 & 0 & & \\
\hline 2 & 22 & 0 & 0 & 0 & & \\
\hline 3 & 24 & 0 & 0 & 0 & & \\
\hline 4 & 24 & 0 & 0 & 0 & & \\
\hline 5 & 26 & 0 & 0 & 0 & & \\
\hline 6 & 26 & 0 & 0 & 0 & & \\
\hline 7 & 28 & 0 & 0 & 0 & & \\
\hline 8 & 28 & 0 & 0 & 0 & & \\
\hline 9 & 30 & 0 & 0 & 0 & & \\
\hline 10 & 30 & 0 & 0 & 0 & & \\
\hline 11 & 32 & 0 & 0 & 0 & & \\
\hline 12 & 32 & 0 & 0 & 0 & & \\
\hline 13 & 34 & 0 & 0 & 0 & & \\
\hline 14 & 34 & 0 & 0 & 0 & & \\
\hline 15 & 36 & 0 & 0 & 0 & & \\
\hline 16 & 36 & 0 & 0 & 0 & & \\
\hline 17 & 38 & 0 & 0 & 0 & & \\
\hline 18 & 38 & 0 & 0 & 0 & & \\
\hline 19 & 40 & 0 & 0 & 0 & & \\
\hline 20 & 40 & 0 & 0 & 0 & & \\
\hline Yield & $0 \%$ & & & & & \\
\hline
\end{tabular}




\begin{tabular}{|c|c|c|c|c|c|c|}
\hline Concentration & $21 \%$ & & & & & \\
\hline Feed rate & $\begin{array}{c}15 \\
\mathrm{~mm} / \mathrm{s}\end{array}$ & & & & & \\
\hline Needle Gauge & 32 & & & & & \\
\hline Dispense Time & $0.02 \mathrm{~s}$ & & & & & \\
\hline \multicolumn{7}{|l|}{ Short to Long } \\
\hline Fiber \# & $\begin{array}{c}\text { Length } \\
(\mathrm{mm})\end{array}$ & $\begin{array}{c}\text { Leading } \\
\text { Diameter } \\
(\mu \mathrm{m})\end{array}$ & $\begin{array}{c}\text { Middle } \\
\text { Diameter } \\
(\mu \mathrm{m})\end{array}$ & $\begin{array}{c}\text { Terminating } \\
\text { Diameter } \\
(\mu \mathrm{m})\end{array}$ & $\begin{array}{c}\text { Average } \\
\text { Diameter } \\
(\mu \mathrm{m})\end{array}$ & $\begin{array}{c}\text { Standard } \\
\text { Deviation } \\
(\mu \mathrm{m})\end{array}$ \\
\hline 1 & 22 & 0 & 0 & 0 & & \\
\hline 2 & 22 & 0 & 0 & 0 & & \\
\hline 3 & 24 & 0 & 0 & 0 & & \\
\hline 4 & 24 & 0 & 0 & 0 & & \\
\hline 5 & 26 & 0 & 0 & 0 & & \\
\hline 6 & 26 & 0 & 0 & 0 & & \\
\hline 7 & 28 & 0 & 0 & 0 & & \\
\hline 8 & 28 & 0 & 0 & 0 & & \\
\hline 9 & 30 & 0 & 0 & 0 & & \\
\hline 10 & 30 & 0 & 0 & 0 & & \\
\hline 11 & 32 & 0 & 0 & 0 & & \\
\hline 12 & 32 & 0 & 0 & 0 & & \\
\hline 13 & 34 & 0 & 0 & 0 & & \\
\hline 14 & 34 & 0 & 0 & 0 & & \\
\hline 15 & 36 & 0 & 0 & 0 & & \\
\hline 16 & 36 & 0 & 0 & 0 & & \\
\hline 17 & 38 & 0 & 0 & 0 & & \\
\hline 18 & 38 & 0 & 0 & 0 & & \\
\hline 19 & 40 & 0 & 0 & 0 & & \\
\hline 20 & 40 & 0 & 0 & 0 & & \\
\hline Yield & $0 \%$ & & & & & \\
\hline
\end{tabular}




\section{APPENDIX D}

\section{PMMA Fiber Characterization Data}

The following data is the fibers' diameter measured using SEM at different surrounding temperature ranging from $70^{\circ} \mathrm{F}$ to $100^{\circ} \mathrm{F}$. In these tables, each fiber was measured three times: two were around $200 \mu \mathrm{m}$ away from the initiating and terminating droplet, one was at the middle location of the fiber to make sure the fiber was uniform. The experimental controlling factors including temperature, polymer solutions concentration, dispense time, feed rates, and need gauge were given before each data set. In the data set, zero means the fiber was broken. And yields were calculated at the end of each data set. 


\begin{tabular}{|c|c|c|c|c|c|c|}
\hline Concentration & $23 \%$ & & & & & \\
\hline Temperature & $70^{\circ} \mathrm{F}$ & & & & & \\
\hline Feed rate & $\begin{array}{c}22.5 \\
\mathrm{~mm} / \mathrm{s}\end{array}$ & & & & & \\
\hline Needle Gauge & 25 & & & & & \\
\hline Dispense Time & $0.02 \mathrm{~s}$ & & & & & \\
\hline \multicolumn{7}{|l|}{ Short to Long } \\
\hline Fiber \# & $\begin{array}{c}\text { Length } \\
(\mathrm{mm})\end{array}$ & $\begin{array}{c}\text { Leading } \\
\text { Diameter } \\
(\mu \mathrm{m})\end{array}$ & $\begin{array}{c}\text { Middle } \\
\text { Diameter } \\
(\mu \mathrm{m})\end{array}$ & $\begin{array}{c}\text { Terminating } \\
\text { Diameter } \\
(\mu \mathrm{m})\end{array}$ & $\begin{array}{c}\text { Average } \\
\text { Diameter } \\
(\mu \mathrm{m})\end{array}$ & $\begin{array}{c}\text { Standard } \\
\text { Deviation } \\
(\mu \mathrm{m})\end{array}$ \\
\hline 1 & 22 & 0 & 0 & 0 & 0.00 & 0.00 \\
\hline 2 & 22 & 0 & 0 & 0 & 0.00 & 0.00 \\
\hline 3 & 24 & 0.4381 & 0.3969 & 0.491 & 0.44 & 0.04 \\
\hline 4 & 24 & 1.828 & 0.4734 & 0.3661 & 0.89 & 0.67 \\
\hline 5 & 26 & 1.056 & 0.3459 & 0.3897 & 0.60 & 0.32 \\
\hline 6 & 26 & 0.3744 & 0.3722 & 0.3027 & 0.35 & 0.03 \\
\hline 7 & 28 & 0.2992 & 0.388 & 0.3947 & 0.36 & 0.04 \\
\hline 8 & 28 & 0 & 0 & 0 & 0.00 & 0.00 \\
\hline 9 & 30 & 0.2791 & 0.4561 & 0.6034 & 0.45 & 0.13 \\
\hline 10 & 30 & 0.9771 & 0.4253 & 1.115 & 0.84 & 0.30 \\
\hline 11 & 32 & 0.5418 & 0.4986 & 1.604 & 0.88 & 0.51 \\
\hline 12 & 32 & 0 & 0 & 0 & 0.00 & 0.00 \\
\hline 13 & 34 & 0 & 0 & 0 & 0.00 & 0.00 \\
\hline 14 & 34 & 0 & 0 & 0 & 0.00 & 0.00 \\
\hline 15 & 36 & 0 & 0 & 0 & 0.00 & 0.00 \\
\hline 16 & 36 & 0 & 0 & 0 & 0.00 & 0.00 \\
\hline 17 & 38 & 0 & 0 & 0 & 0.00 & 0.00 \\
\hline 18 & 38 & 0 & 0 & 0 & 0.00 & 0.00 \\
\hline 19 & 40 & 0 & 0 & 0 & 0.00 & 0.00 \\
\hline 20 & 40 & 0 & 0 & 0 & 0.00 & 0.00 \\
\hline Yield & $40 \%$ & & & & & \\
\hline
\end{tabular}




\begin{tabular}{|c|c|c|c|c|c|c|}
\hline Concentration & $23 \%$ & & & & & \\
\hline Temperature & $70^{\circ} \mathrm{F}$ & & & & & \\
\hline Feed rate & $\begin{array}{c}15 \\
\mathrm{~mm} / \mathrm{s}\end{array}$ & & & & & \\
\hline Needle Gauge & 25 & & & & & \\
\hline Dispense Time & $0.02 \mathrm{~s}$ & & & & & \\
\hline \multicolumn{7}{|l|}{ Short to Long } \\
\hline Fiber \# & $\begin{array}{c}\text { Length } \\
(\mathrm{mm})\end{array}$ & $\begin{array}{c}\text { Leading } \\
\text { Diameter } \\
(\mu \mathrm{m})\end{array}$ & $\begin{array}{c}\text { Middle } \\
\text { Diameter } \\
(\mu \mathrm{m})\end{array}$ & $\begin{array}{c}\text { Terminating } \\
\text { Diameter } \\
(\mu \mathrm{m})\end{array}$ & $\begin{array}{c}\text { Average } \\
\text { Diameter } \\
(\mu \mathrm{m})\end{array}$ & $\begin{array}{c}\text { Standard } \\
\text { Deviation } \\
(\mu \mathrm{m})\end{array}$ \\
\hline 1 & 22 & 0.6598 & 0.3298 & 0.9383 & 0.64 & 0.25 \\
\hline 2 & 22 & 0.3441 & 0.2975 & 0.9542 & 0.53 & 0.30 \\
\hline 3 & 24 & 0.3663 & 0.56 & 1.183 & 0.70 & 0.35 \\
\hline 4 & 24 & 0.2135 & 0.3592 & 1.076 & 0.55 & 0.38 \\
\hline 5 & 26 & 0.3723 & 0.2835 & 1.185 & 0.61 & 0.41 \\
\hline 6 & 26 & 0 & 0 & 0 & 0.00 & 0.00 \\
\hline 7 & 28 & 0 & 0 & 0 & 0.00 & 0.00 \\
\hline 8 & 28 & 0 & 0 & 0 & 0.00 & 0.00 \\
\hline 9 & 30 & 0 & 0 & 0 & 0.00 & 0.00 \\
\hline 10 & 30 & 0 & 0 & 0 & 0.00 & 0.00 \\
\hline 11 & 32 & 0 & 0 & 0 & 0.00 & 0.00 \\
\hline 12 & 32 & 0 & 0 & 0 & 0.00 & 0.00 \\
\hline 13 & 34 & 0 & 0 & 0 & 0.00 & 0.00 \\
\hline 14 & 34 & 0 & 0 & 0 & 0.00 & 0.00 \\
\hline 15 & 36 & 0 & 0 & 0 & 0.00 & 0.00 \\
\hline 16 & 36 & 0 & 0 & 0 & 0.00 & 0.00 \\
\hline 17 & 38 & 0 & 0 & 0 & 0.00 & 0.00 \\
\hline 18 & 38 & 0 & 0 & 0 & 0.00 & 0.00 \\
\hline 19 & 40 & 0 & 0 & 0 & 0.00 & 0.00 \\
\hline 20 & 40 & 0 & 0 & 0 & 0.00 & 0.00 \\
\hline Yield & $25 \%$ & & & & & \\
\hline
\end{tabular}




\begin{tabular}{|c|c|c|c|c|c|c|}
\hline Concentration & $23 \%$ & & & & & \\
\hline Temperature & $70^{\circ} \mathrm{F}$ & & & & & \\
\hline Feed rate & $\begin{array}{c}22.5 \\
\mathrm{~mm} / \mathrm{s}\end{array}$ & & & & & \\
\hline Needle Gauge & 27 & & & & & \\
\hline Dispense Time & $0.02 \mathrm{~s}$ & & & & & \\
\hline \multicolumn{7}{|l|}{ Short to Long } \\
\hline Fiber \# & $\begin{array}{c}\text { Length } \\
(\mathrm{mm})\end{array}$ & $\begin{array}{c}\text { Leading } \\
\text { Diameter } \\
(\mu \mathrm{m})\end{array}$ & $\begin{array}{c}\text { Middle } \\
\text { Diameter } \\
(\mu \mathrm{m})\end{array}$ & $\begin{array}{c}\text { Terminating } \\
\text { Diameter } \\
(\mu \mathrm{m})\end{array}$ & $\begin{array}{c}\text { Average } \\
\text { Diameter } \\
(\mu \mathrm{m})\end{array}$ & $\begin{array}{c}\text { Standard } \\
\text { Deviation } \\
(\mu \mathrm{m})\end{array}$ \\
\hline 1 & 22 & 4.4 & 1.517 & 2.357 & 2.76 & 1.21 \\
\hline 2 & 22 & 0 & 0 & 0 & 0.00 & 0.00 \\
\hline 3 & 24 & 0 & 0 & 0 & 0.00 & 0.00 \\
\hline 4 & 24 & 0 & 0 & 0 & 0.00 & 0.00 \\
\hline 5 & 26 & 1.818 & 0.6097 & 0.6234 & 1.02 & 0.57 \\
\hline 6 & 26 & 1.524 & 0.5558 & 0.6295 & 0.90 & 0.44 \\
\hline 7 & 28 & 0.3563 & 0.7021 & 0.8213 & 0.63 & 0.20 \\
\hline 8 & 28 & 0.1948 & 0.1844 & 1.301 & 0.56 & 0.52 \\
\hline 9 & 30 & 0.4745 & 1.536 & 0.9328 & 0.98 & 0.43 \\
\hline 10 & 30 & 1.301 & 0.4925 & 0.740 & 0.84 & 0.34 \\
\hline 11 & 32 & 0 & 0 & 0 & 0.00 & 0.00 \\
\hline 12 & 32 & 2.388 & 0.7582 & 1.538 & 1.56 & 0.67 \\
\hline 13 & 34 & 0 & 0 & 0 & 0.00 & 0.00 \\
\hline 14 & 34 & 0.7081 & 0.1891 & 1.673 & 0.86 & 0.61 \\
\hline 15 & 36 & 0 & 0 & 0 & 0.00 & 0.00 \\
\hline 16 & 36 & 1.2 & 0.8052 & 0.5117 & 0.84 & 0.28 \\
\hline 17 & 38 & 2.926 & 0.8488 & 1.475 & 1.75 & 0.87 \\
\hline 18 & 38 & 2.533 & 1.191 & 2.428 & 2.05 & 0.61 \\
\hline 19 & 40 & 0 & 0 & 0 & 0.00 & 0.00 \\
\hline 20 & 40 & 0 & 0 & 0 & 0.00 & 0.00 \\
\hline Yield & $60 \%$ & & & & & \\
\hline
\end{tabular}




\begin{tabular}{|c|c|c|c|c|c|c|}
\hline Concentration & $23 \%$ & & & & & \\
\hline Temperature & $70^{\circ} \mathrm{F}$ & & & & & \\
\hline Feed rate & $\begin{array}{c}15 \\
\mathrm{~mm} / \mathrm{s}\end{array}$ & & & & & \\
\hline Needle Gauge & 27 & & & & & \\
\hline Dispense Time & $0.02 \mathrm{~s}$ & & & & & \\
\hline \multicolumn{7}{|l|}{ Short to Long } \\
\hline Fiber \# & $\begin{array}{c}\text { Length } \\
(\mathrm{mm})\end{array}$ & $\begin{array}{c}\text { Leading } \\
\text { Diameter } \\
(\mu \mathrm{m})\end{array}$ & $\begin{array}{c}\text { Middle } \\
\text { Diameter } \\
(\mu \mathrm{m})\end{array}$ & $\begin{array}{c}\text { Terminating } \\
\text { Diameter } \\
(\mu \mathrm{m})\end{array}$ & $\begin{array}{c}\text { Average } \\
\text { Diameter } \\
(\mu \mathrm{m})\end{array}$ & $\begin{array}{c}\text { Standard } \\
\text { Deviation } \\
(\mu \mathrm{m})\end{array}$ \\
\hline 1 & 22 & 1.449 & 1.070 & 1.308 & 1.28 & 0.16 \\
\hline 2 & 22 & 0.8419 & 0.7611 & 1.66 & 1.09 & 0.41 \\
\hline 3 & 24 & 0.8411 & 0.9628 & 0.758 & 0.85 & 0.08 \\
\hline 4 & 24 & 0.7707 & 0.6254 & 1.328 & 0.91 & 0.30 \\
\hline 5 & 26 & 1.443 & 0.7575 & 0.9569 & 1.05 & 0.29 \\
\hline 6 & 26 & 0.2671 & 1.667 & 0.7055 & 0.88 & 0.58 \\
\hline 7 & 28 & 0.5961 & 0.5199 & 0.9348 & 0.68 & 0.18 \\
\hline 8 & 28 & 1.847 & 1.180 & 1.492 & 1.51 & 0.27 \\
\hline 9 & 30 & 0.9494 & 0.951 & 1.195 & 1.03 & 0.12 \\
\hline 10 & 30 & 1.497 & 1.170 & 1.250 & 1.31 & 0.14 \\
\hline 11 & 32 & 1.665 & 1.004 & 1.236 & 1.30 & 0.27 \\
\hline 12 & 32 & 3.536 & 1.237 & 2.730 & 2.50 & 0.95 \\
\hline 13 & 34 & 0 & 0 & 0 & 0.00 & 0.00 \\
\hline 14 & 34 & 0 & 0 & 0 & 0.00 & 0.00 \\
\hline 15 & 36 & 0 & 0 & 0 & 0.00 & 0.00 \\
\hline 16 & 36 & 2.431 & 0.9452 & 2.115 & 1.83 & 0.64 \\
\hline 17 & 38 & 2.506 & 2.1 & 1.114 & 1.91 & 0.58 \\
\hline 18 & 38 & 0 & 0 & 0 & 0.00 & 0.00 \\
\hline 19 & 40 & 0 & 0 & 0 & 0.00 & 0.00 \\
\hline 20 & 40 & 1.474 & 1.342 & 2.476 & 1.76 & 0.51 \\
\hline Yield & $75 \%$ & & & & & \\
\hline
\end{tabular}




\begin{tabular}{|c|c|c|c|c|c|c|}
\hline Concentration & $23 \%$ & & & & & \\
\hline Temperature & $80^{\circ} \mathrm{F}$ & & & & & \\
\hline Feed rate & $\begin{array}{c}22.5 \\
\mathrm{~mm} / \mathrm{s}\end{array}$ & & & & & \\
\hline Needle Gauge & 25 & & & & & \\
\hline Dispense Time & $0.02 \mathrm{~s}$ & & & & & \\
\hline \multicolumn{7}{|l|}{ Short to Long } \\
\hline Fiber \# & $\begin{array}{c}\text { Length } \\
(\mathrm{mm})\end{array}$ & $\begin{array}{c}\text { Leading } \\
\text { Diameter } \\
(\mu \mathrm{m})\end{array}$ & $\begin{array}{c}\text { Middle } \\
\text { Diameter } \\
(\mu \mathrm{m})\end{array}$ & $\begin{array}{c}\text { Terminating } \\
\text { Diameter } \\
(\mu \mathrm{m})\end{array}$ & $\begin{array}{c}\text { Average } \\
\text { Diameter } \\
(\mu \mathrm{m})\end{array}$ & $\begin{array}{c}\text { Standard } \\
\text { Deviation } \\
(\mu \mathrm{m})\end{array}$ \\
\hline 1 & 22 & 0 & 0 & 0 & 0 & 0 \\
\hline 2 & 22 & 2.45 & 4.765 & 9.26 & 5.49 & 2.83 \\
\hline 3 & 24 & 3.227 & 2.842 & 5.169 & 3.75 & 1.02 \\
\hline 4 & 24 & 2.447 & 5.408 & 12.62 & 6.83 & 4.27 \\
\hline 5 & 26 & 2.557 & 3.732 & 21.37 & 9.22 & 8.60 \\
\hline 6 & 26 & 2.619 & 3.371 & 4.124 & 3.37 & 0.61 \\
\hline 7 & 28 & 3.102 & 3.751 & 9.957 & 5.60 & 3.09 \\
\hline 8 & 28 & 2.073 & 5.416 & 1.807 & 3.10 & 1.64 \\
\hline 9 & 30 & 1.985 & 6.125 & 5.175 & 4.43 & 1.77 \\
\hline 10 & 30 & 0 & 0 & 0 & 0 & 0 \\
\hline 11 & 32 & 0 & 0 & 0 & 0 & 0 \\
\hline 12 & 32 & 4.504 & 2.121 & 19.31 & 8.65 & 7.60 \\
\hline 13 & 34 & 3.786 & 2.809 & 18.71 & 8.44 & 7.28 \\
\hline 14 & 34 & 0 & 0 & 0 & 0 & 0 \\
\hline 15 & 36 & 0 & 0 & 0 & 0 & 0 \\
\hline 16 & 36 & 0 & 0 & 0 & 0 & 0 \\
\hline 17 & 38 & 0 & 0 & 0 & 0 & 0 \\
\hline 18 & 38 & 0 & 0 & 0 & 0 & 0 \\
\hline 19 & 40 & 0 & 0 & 0 & 0 & 0 \\
\hline 20 & 40 & 0 & 0 & 0 & 0 & 0 \\
\hline Yield & $50 \%$ & & & & & \\
\hline
\end{tabular}




\begin{tabular}{|c|c|c|c|c|c|c|}
\hline Concentration & $23 \%$ & & & & & \\
\hline Temperature & $80^{\circ} \mathrm{F}$ & & & & & \\
\hline Feed rate & $\begin{array}{c}15 \\
\mathrm{~mm} / \mathrm{s}\end{array}$ & & & & & \\
\hline Needle Gauge & 25 & & & & & \\
\hline Dispense Time & $0.02 \mathrm{~s}$ & & & & & \\
\hline \multicolumn{7}{|l|}{ Short to Long } \\
\hline Fiber \# & $\begin{array}{c}\text { Length } \\
(\mathrm{mm})\end{array}$ & $\begin{array}{c}\text { Leading } \\
\text { Diameter } \\
(\mu \mathrm{m})\end{array}$ & $\begin{array}{c}\text { Middle } \\
\text { Diameter } \\
(\mu \mathrm{m})\end{array}$ & $\begin{array}{c}\text { Terminating } \\
\text { Diameter } \\
(\mu \mathrm{m})\end{array}$ & $\begin{array}{c}\text { Average } \\
\text { Diameter } \\
(\mu \mathrm{m})\end{array}$ & $\begin{array}{c}\text { Standard } \\
\text { Deviation } \\
(\mu \mathrm{m})\end{array}$ \\
\hline 1 & 22 & 0 & 0 & 0 & 0 & 0 \\
\hline 2 & 22 & 0 & 0 & 0 & 0 & 0 \\
\hline 3 & 24 & 9.917 & 6.905 & 2.955 & 6.59 & 2.85 \\
\hline 4 & 24 & 10.05 & 13.86 & 6.818 & 10.24 & 2.88 \\
\hline 5 & 26 & 0 & 0 & 0 & 0 & 0 \\
\hline 6 & 26 & 0 & 0 & 0 & 0 & 0 \\
\hline 7 & 28 & 1.839 & 2.421 & 7.327 & 3.86 & 2.46 \\
\hline 8 & 28 & 0 & 0 & 0 & 0 & 0 \\
\hline 9 & 30 & 7.899 & 6.998 & 8.275 & 7.72 & 0.54 \\
\hline 10 & 30 & 0 & 0 & 0 & 0 & 0 \\
\hline 11 & 32 & 0 & 0 & 0 & 0 & 0 \\
\hline 12 & 32 & 0 & 0 & 0 & 0 & 0 \\
\hline 13 & 34 & 0 & 0 & 0 & 0 & 0 \\
\hline 14 & 34 & 0 & 0 & 0 & 0 & 0 \\
\hline 15 & 36 & 0 & 0 & 0 & 0 & 0 \\
\hline 16 & 36 & 0 & 0 & 0 & 0 & 0 \\
\hline 17 & 38 & 0 & 0 & 0 & 0 & 0 \\
\hline 18 & 38 & 0 & 0 & 0 & 0 & 0 \\
\hline 19 & 40 & 0 & 0 & 0 & 0 & 0 \\
\hline 20 & 40 & 0 & 0 & 0 & 0 & 0 \\
\hline Yield & $20 \%$ & & & & & \\
\hline
\end{tabular}




\begin{tabular}{|c|c|c|c|c|c|c|}
\hline Concentration & $23 \%$ & & & & & \\
\hline Temperature & $80^{\circ} \mathrm{F}$ & & & & & \\
\hline Feed rate & $\begin{array}{c}22.5 \\
\mathrm{~mm} / \mathrm{s}\end{array}$ & & & & & \\
\hline Needle Gauge & 27 & & & & & \\
\hline Dispense Time & $0.02 \mathrm{~s}$ & & & & & \\
\hline \multicolumn{7}{|l|}{ Short to Long } \\
\hline Fiber \# & $\begin{array}{c}\text { Length } \\
(\mathrm{mm})\end{array}$ & $\begin{array}{l}\text { Leading } \\
\text { Diameter } \\
(\mu \mathrm{m})\end{array}$ & $\begin{array}{c}\text { Middle } \\
\text { Diameter } \\
(\mu \mathrm{m})\end{array}$ & $\begin{array}{c}\text { Terminating } \\
\text { Diameter } \\
(\mu \mathrm{m})\end{array}$ & $\begin{array}{c}\text { Average } \\
\text { Diameter } \\
(\mu \mathrm{m})\end{array}$ & $\begin{array}{c}\text { Standard } \\
\text { Deviation } \\
(\mu \mathrm{m})\end{array}$ \\
\hline 1 & 22 & 0 & 0 & 0 & 0 & 0 \\
\hline 2 & 22 & 0 & 0 & 0 & 0 & 0 \\
\hline 3 & 24 & 0 & 0 & 0 & 0 & 0 \\
\hline 4 & 24 & 2.866 & 6.920 & 13.53 & 7.77 & 4.40 \\
\hline 5 & 26 & 9.105 & 8.15 & 14.1 & 10.45 & 2.61 \\
\hline 6 & 26 & 3.045 & 8.151 & 3.027 & 4.74 & 2.41 \\
\hline 7 & 28 & 8.837 & 6.635 & 3.190 & 6.22 & 2.32 \\
\hline 8 & 28 & 6.565 & 5.563 & 5.765 & 5.96 & 0.43 \\
\hline 9 & 30 & 4.120 & 5.195 & 10.68 & 6.67 & 2.87 \\
\hline 10 & 30 & 7.609 & 16.75 & 5.528 & 9.96 & 4.87 \\
\hline 11 & 32 & 4.695 & 13.13 & 6.777 & 8.20 & 3.59 \\
\hline 12 & 32 & 4.442 & 5.105 & 5.289 & 4.95 & 0.36 \\
\hline 13 & 34 & 12.66 & 7.982 & 15.71 & 12.12 & 3.18 \\
\hline 14 & 34 & 6.023 & 9.438 & 7.491 & 7.65 & 1.40 \\
\hline 15 & 36 & 0 & 0 & 0 & 0 & 0 \\
\hline 16 & 36 & 0 & 0 & 0 & 0 & 0 \\
\hline 17 & 38 & 0 & 0 & 0 & 0 & 0 \\
\hline 18 & 38 & 0 & 0 & 0 & 0 & 0 \\
\hline 19 & 40 & 6.906 & 6.986 & 5.008 & 6.30 & 0.91 \\
\hline 20 & 40 & 0 & 0 & 0 & 0 & 0 \\
\hline Yield & $60 \%$ & & & & & \\
\hline
\end{tabular}




\begin{tabular}{|c|c|c|c|c|c|c|}
\hline Concentration & $23 \%$ & & & & & \\
\hline Temperature & $80^{\circ} \mathrm{F}$ & & & & & \\
\hline Feed rate & $\begin{array}{c}15 \\
\mathrm{~mm} / \mathrm{s}\end{array}$ & & & & & \\
\hline Needle Gauge & 27 & & & & & \\
\hline Dispense Time & $0.02 \mathrm{~s}$ & & & & & \\
\hline \multicolumn{7}{|l|}{ Short to Long } \\
\hline Fiber \# & $\begin{array}{c}\text { Length } \\
(\mathrm{mm})\end{array}$ & $\begin{array}{c}\text { Leading } \\
\text { Diameter } \\
(\mu \mathrm{m})\end{array}$ & $\begin{array}{c}\text { Middle } \\
\text { Diameter } \\
(\mu \mathrm{m})\end{array}$ & $\begin{array}{c}\text { Terminating } \\
\text { Diameter } \\
(\mu \mathrm{m})\end{array}$ & $\begin{array}{c}\text { Average } \\
\text { Diameter } \\
(\mu \mathrm{m})\end{array}$ & $\begin{array}{c}\text { Standard } \\
\text { Deviation } \\
(\mu \mathrm{m})\end{array}$ \\
\hline 1 & 22 & 0 & 0 & 0 & 0 & 0 \\
\hline 2 & 22 & 6.789 & 13.24 & 2.287 & 7.44 & 4.50 \\
\hline 3 & 24 & 9.725 & 21.18 & 26.08 & 19.00 & 6.85 \\
\hline 4 & 24 & 9.846 & 9.333 & 13.19 & 10.79 & 1.71 \\
\hline 5 & 26 & 12.55 & 10.67 & 9.694 & 10.97 & 1.19 \\
\hline 6 & 26 & 6.032 & 15.69 & 6.795 & 9.51 & 4.38 \\
\hline 7 & 28 & 10.02 & 10.24 & 14.27 & 11.51 & 1.95 \\
\hline 8 & 28 & 0 & 0 & 0 & 0 & 0 \\
\hline 9 & 30 & 0 & 0 & 0 & 0 & 0 \\
\hline 10 & 30 & 0 & 0 & 0 & 0 & 0 \\
\hline 11 & 32 & 0 & 0 & 0 & 0 & 0 \\
\hline 12 & 32 & 0 & 0 & 0 & 0 & 0 \\
\hline 13 & 34 & 0 & 0 & 0 & 0 & 0 \\
\hline 14 & 34 & 0 & 0 & 0 & 0 & 0 \\
\hline 15 & 36 & 0 & 0 & 0 & 0 & 0 \\
\hline 16 & 36 & 0 & 0 & 0 & 0 & 0 \\
\hline 17 & 38 & 0 & 0 & 0 & 0 & 0 \\
\hline 18 & 38 & 0 & 0 & 0 & 0 & 0 \\
\hline 19 & 40 & 0 & 0 & 0 & 0 & 0 \\
\hline 20 & 40 & 0 & 0 & 0 & 0 & 0 \\
\hline Yield & $30 \%$ & & & & & \\
\hline
\end{tabular}




\begin{tabular}{|c|c|c|c|c|c|c|}
\hline Concentration & $23 \%$ & & & & & \\
\hline Temperature & $90^{\circ} \mathrm{F}$ & & & & & \\
\hline Feed rate & $\begin{array}{c}22.5 \\
\mathrm{~mm} / \mathrm{s}\end{array}$ & & & & & \\
\hline Needle Gauge & 25 & & & & & \\
\hline Dispense Time & $0.02 \mathrm{~s}$ & & & & & \\
\hline \multicolumn{7}{|l|}{ Short to Long } \\
\hline Fiber \# & $\begin{array}{c}\text { Length } \\
(\mathrm{mm})\end{array}$ & $\begin{array}{c}\text { Leading } \\
\text { Diameter } \\
(\mu \mathrm{m})\end{array}$ & $\begin{array}{c}\text { Middle } \\
\text { Diameter } \\
(\mu \mathrm{m})\end{array}$ & $\begin{array}{c}\text { Terminating } \\
\text { Diameter } \\
(\mu \mathrm{m})\end{array}$ & $\begin{array}{c}\text { Average } \\
\text { Diameter } \\
(\mu \mathrm{m})\end{array}$ & $\begin{array}{c}\text { Standard } \\
\text { Deviation } \\
(\mu \mathrm{m})\end{array}$ \\
\hline 1 & 22 & 0 & 0 & 0 & 0 & 0 \\
\hline 2 & 22 & 9.248 & 4.469 & 5.980 & 6.57 & 1.99 \\
\hline 3 & 24 & 2.419 & 2.848 & 13.24 & 6.17 & 5.00 \\
\hline 4 & 24 & 3.335 & 7.574 & 7.132 & 6.01 & 1.90 \\
\hline 5 & 26 & 6.165 & 10.56 & 4.983 & 7.24 & 2.40 \\
\hline 6 & 26 & 1.918 & 3.886 & 5.190 & 3.66 & 1.34 \\
\hline 7 & 28 & 4.205 & 6.750 & 2.756 & 4.57 & 1.65 \\
\hline 8 & 28 & 1.847 & 4.493 & 5.199 & 3.85 & 1.44 \\
\hline 9 & 30 & 6.829 & 10.60 & 4.5 & 7.31 & 2.51 \\
\hline 10 & 30 & 7.150 & 6.094 & 6.355 & 6.53 & 0.45 \\
\hline 11 & 32 & 2.892 & 2.898 & 6.562 & 4.12 & 1.73 \\
\hline 12 & 32 & 9.211 & 5.804 & 5.050 & 6.69 & 1.81 \\
\hline 13 & 34 & 16.67 & 12.61 & 15.7 & 14.99 & 1.73 \\
\hline 14 & 34 & 3.583 & 4.239 & 12.35 & 6.72 & 3.99 \\
\hline 15 & 36 & 8.066 & 5.615 & 6.760 & 6.81 & 1.00 \\
\hline 16 & 36 & 8.424 & 3.783 & 3.248 & 5.15 & 2.32 \\
\hline 17 & 38 & 0 & 0 & 0 & 0 & 0 \\
\hline 18 & 38 & 3.557 & 2.271 & 2.792 & 2.87 & 0.53 \\
\hline 19 & 40 & 1.985 & 3.101 & 5.742 & 3.61 & 1.58 \\
\hline 20 & 40 & 3.822 & 3.127 & 7.808 & 4.92 & 2.06 \\
\hline Yield & $90 \%$ & & & & & \\
\hline
\end{tabular}




\begin{tabular}{|c|c|c|c|c|c|c|}
\hline Concentration & $23 \%$ & & & & & \\
\hline Temperature & $90^{\circ} \mathrm{F}$ & & & & & \\
\hline Feed rate & $\begin{array}{c}15 \\
\mathrm{~mm} / \mathrm{s}\end{array}$ & & & & & \\
\hline Needle Gauge & 25 & & & & & \\
\hline Dispense Time & $0.02 \mathrm{~s}$ & & & & & \\
\hline \multicolumn{7}{|l|}{ Short to Long } \\
\hline Fiber \# & $\begin{array}{c}\text { Length } \\
(\mathrm{mm})\end{array}$ & $\begin{array}{c}\text { Leading } \\
\text { Diameter } \\
(\mu \mathrm{m})\end{array}$ & $\begin{array}{c}\text { Middle } \\
\text { Diameter } \\
(\mu \mathrm{m})\end{array}$ & $\begin{array}{c}\text { Terminating } \\
\text { Diameter } \\
(\mu \mathrm{m})\end{array}$ & $\begin{array}{c}\text { Average } \\
\text { Diameter } \\
(\mu \mathrm{m})\end{array}$ & $\begin{array}{c}\text { Standard } \\
\text { Deviation } \\
(\mu \mathrm{m})\end{array}$ \\
\hline 1 & 22 & 0 & 0 & 0 & 0 & 0 \\
\hline 2 & 22 & 0 & 0 & 0 & 0 & 0 \\
\hline 3 & 24 & 6.328 & 14.13 & 8.892 & 9.78 & 3.25 \\
\hline 4 & 24 & 4.613 & 20.67 & 15.24 & 13.51 & 6.67 \\
\hline 5 & 26 & 4.869 & 3.309 & 7.964 & 5.38 & 1.93 \\
\hline 6 & 26 & 2.859 & 3.647 & 6.844 & 4.45 & 1.72 \\
\hline 7 & 28 & 2.207 & 2.171 & 3.005 & 2.46 & 0.38 \\
\hline 8 & 28 & 2.166 & 5.124 & 7.489 & 4.93 & 2.18 \\
\hline 9 & 30 & 1.525 & 2.367 & 3.442 & 2.44 & 0.78 \\
\hline 10 & 30 & 2.867 & 1.707 & 11.07 & 5.21 & 4.17 \\
\hline 11 & 32 & 0 & 0 & 0 & 0 & 0 \\
\hline 12 & 32 & 0 & 0 & 0 & 0 & 0 \\
\hline 13 & 34 & 0 & 0 & 0 & 0 & 0 \\
\hline 14 & 34 & 0 & 0 & 0 & 0 & 0 \\
\hline 15 & 36 & 0 & 0 & 0 & 0 & 0 \\
\hline 16 & 36 & 8.895 & 4.377 & 5.508 & 6.26 & 1.92 \\
\hline 17 & 38 & 6.710 & 5.929 & 3.477 & 5.37 & 1.38 \\
\hline 18 & 38 & 4.402 & 2.214 & 1.576 & 2.73 & 1.21 \\
\hline 19 & 40 & 0 & 0 & 0 & 0 & 0 \\
\hline 20 & 40 & 0 & 0 & 0 & 0 & 0 \\
\hline Yield & $55 \%$ & & & & & \\
\hline
\end{tabular}




\begin{tabular}{|c|c|c|c|c|c|c|}
\hline Concentration & $23 \%$ & & & & & \\
\hline Temperature & $90^{\circ} \mathrm{F}$ & & & & & \\
\hline Feed rate & $\begin{array}{c}22.5 \\
\mathrm{~mm} / \mathrm{s}\end{array}$ & & & & & \\
\hline Needle Gauge & 27 & & & & & \\
\hline Dispense Time & $0.02 \mathrm{~s}$ & & & & & \\
\hline \multicolumn{7}{|l|}{ Short to Long } \\
\hline Fiber \# & $\begin{array}{c}\text { Length } \\
(\mathrm{mm})\end{array}$ & $\begin{array}{c}\text { Leading } \\
\text { Diameter } \\
(\mu \mathrm{m})\end{array}$ & $\begin{array}{c}\text { Middle } \\
\text { Diameter } \\
(\mu \mathrm{m})\end{array}$ & $\begin{array}{c}\text { Terminating } \\
\text { Diameter } \\
(\mu \mathrm{m})\end{array}$ & $\begin{array}{c}\text { Average } \\
\text { Diameter } \\
(\mu \mathrm{m})\end{array}$ & $\begin{array}{c}\text { Standard } \\
\text { Deviation } \\
(\mu \mathrm{m})\end{array}$ \\
\hline 1 & 22 & 0 & 0 & 0 & 0 & 0 \\
\hline 2 & 22 & 0 & 0 & 0 & 0 & 0 \\
\hline 3 & 24 & 16.27 & 10.55 & 6.826 & 11.22 & 3.88 \\
\hline 4 & 24 & 0 & 0 & 0 & 0 & 0 \\
\hline 5 & 26 & 0 & 0 & 0 & 0 & 0 \\
\hline 6 & 26 & 0 & 0 & 0 & 0 & 0 \\
\hline 7 & 28 & 0 & 0 & 0 & 0 & 0 \\
\hline 8 & 28 & 0 & 0 & 0 & 0 & 0 \\
\hline 9 & 30 & 0 & 0 & 0 & 0 & 0 \\
\hline 10 & 30 & 0 & 0 & 0 & 0 & 0 \\
\hline 11 & 32 & 0 & 0 & 0 & 0 & 0 \\
\hline 12 & 32 & 0 & 0 & 0 & 0 & 0 \\
\hline 13 & 34 & 0 & 0 & 0 & 0 & 0 \\
\hline 14 & 34 & 10.13 & 26.30 & 20.29 & 18.91 & 6.67 \\
\hline 15 & 36 & 14.24 & 11.21 & 9.059 & 11.50 & 2.13 \\
\hline 16 & 36 & 0 & 0 & 0 & 0 & 0 \\
\hline 17 & 38 & 0 & 0 & 0 & 0 & 0 \\
\hline 18 & 38 & 0 & 0 & 0 & 0 & 0 \\
\hline 19 & 40 & 0 & 0 & 0 & 0 & 0 \\
\hline 20 & 40 & 0 & 0 & 0 & 0 & 0 \\
\hline Yield & $15 \%$ & & & & & \\
\hline
\end{tabular}




\begin{tabular}{|c|c|c|c|c|c|c|}
\hline Concentration & $23 \%$ & & & & & \\
\hline Temperature & $90^{\circ} \mathrm{F}$ & & & & & \\
\hline Feed rate & $\begin{array}{c}15 \\
\mathrm{~mm} / \mathrm{s}\end{array}$ & & & & & \\
\hline Needle Gauge & 27 & & & & & \\
\hline Dispense Time & $0.02 \mathrm{~s}$ & & & & & \\
\hline \multicolumn{7}{|l|}{ Short to Long } \\
\hline Fiber \# & $\begin{array}{c}\text { Length } \\
(\mathrm{mm})\end{array}$ & $\begin{array}{c}\text { Leading } \\
\text { Diameter } \\
(\mu \mathrm{m})\end{array}$ & $\begin{array}{c}\text { Middle } \\
\text { Diameter } \\
(\mu \mathrm{m})\end{array}$ & $\begin{array}{c}\text { Terminating } \\
\text { Diameter } \\
(\mu \mathrm{m})\end{array}$ & $\begin{array}{c}\text { Average } \\
\text { Diameter } \\
(\mu \mathrm{m})\end{array}$ & $\begin{array}{c}\text { Standard } \\
\text { Deviation } \\
(\mu \mathrm{m})\end{array}$ \\
\hline 1 & 22 & 0 & 0 & 0 & 0 & 0 \\
\hline 2 & 22 & 0 & 0 & 0 & 0 & 0 \\
\hline 3 & 24 & 0 & 0 & 0 & 0 & 0 \\
\hline 4 & 24 & 12.76 & 21.35 & 13.10 & 15.74 & 3.97 \\
\hline 5 & 26 & 11.26 & 13.83 & 8.859 & 11.32 & 2.03 \\
\hline 6 & 26 & 0 & 0 & 0 & 0 & 0 \\
\hline 7 & 28 & 0 & 0 & 0 & 0 & 0 \\
\hline 8 & 28 & 0 & 0 & 0 & 0 & 0 \\
\hline 9 & 30 & 0 & 0 & 0 & 0 & 0 \\
\hline 10 & 30 & 0 & 0 & 0 & 0 & 0 \\
\hline 11 & 32 & 0 & 0 & 0 & 0 & 0 \\
\hline 12 & 32 & 0 & 0 & 0 & 0 & 0 \\
\hline 13 & 34 & 0 & 0 & 0 & 0 & 0 \\
\hline 14 & 34 & 0 & 0 & 0 & 0 & 0 \\
\hline 15 & 36 & 0 & 0 & 0 & 0 & 0 \\
\hline 16 & 36 & 11.01 & 6.179 & 6.712 & 7.97 & 2.16 \\
\hline 17 & 38 & 12.43 & 14.87 & 9.307 & 12.20 & 2.28 \\
\hline 18 & 38 & 0 & 0 & 0 & 0 & 0 \\
\hline 19 & 40 & 0 & 0 & 0 & 0 & 0 \\
\hline 20 & 40 & 15.03 & 19.60 & 7.651 & 14.09 & 4.92 \\
\hline Yield & $25 \%$ & & & & & \\
\hline
\end{tabular}




\begin{tabular}{|c|c|c|c|c|c|c|}
\hline Concentration & $23 \%$ & & & & & \\
\hline Temperature & $100^{\circ} \mathrm{F}$ & & & & & \\
\hline Feed rate & $\begin{array}{c}22.5 \\
\mathrm{~mm} / \mathrm{s}\end{array}$ & & & & & \\
\hline Needle Gauge & 25 & & & & & \\
\hline Dispense Time & $0.02 \mathrm{~s}$ & & & & & \\
\hline \multicolumn{7}{|l|}{ Short to Long } \\
\hline Fiber \# & $\begin{array}{c}\text { Length } \\
(\mathrm{mm})\end{array}$ & $\begin{array}{c}\text { Leading } \\
\text { Diameter } \\
(\mu \mathrm{m})\end{array}$ & $\begin{array}{c}\text { Middle } \\
\text { Diameter } \\
(\mu \mathrm{m})\end{array}$ & $\begin{array}{c}\text { Terminating } \\
\text { Diameter } \\
(\mu \mathrm{m})\end{array}$ & $\begin{array}{c}\text { Average } \\
\text { Diameter } \\
(\mu \mathrm{m})\end{array}$ & $\begin{array}{c}\text { Standard } \\
\text { Deviation } \\
(\mu \mathrm{m})\end{array}$ \\
\hline 1 & 22 & 0 & 0 & 0 & 0 & 0 \\
\hline 2 & 22 & 0 & 0 & 0 & 0 & 0 \\
\hline 3 & 24 & 3.094 & 1.942 & 2.055 & 2.36 & 0.52 \\
\hline 4 & 24 & 9.776 & 9.879 & 14.78 & 11.48 & 2.34 \\
\hline 5 & 26 & 3.265 & 4.977 & 2.318 & 3.52 & 1.10 \\
\hline 6 & 26 & 6.493 & 3.613 & 4.482 & 4.86 & 1.21 \\
\hline 7 & 28 & 0 & 0 & 0 & 0 & 0 \\
\hline 8 & 28 & 0 & 0 & 0 & 0 & 0 \\
\hline 9 & 30 & 0 & 0 & 0 & 0 & 0 \\
\hline 10 & 30 & 3.595 & 1.506 & 1.786 & 2.30 & 0.93 \\
\hline 11 & 32 & 0 & 0 & 0 & 0 & 0 \\
\hline 12 & 32 & 0 & 0 & 0 & 0 & 0 \\
\hline 13 & 34 & 4.304 & 5.718 & 4.478 & 4.83 & 0.63 \\
\hline 14 & 34 & 8.592 & 2.247 & 5.120 & 5.32 & 2.59 \\
\hline 15 & 36 & 3.242 & 6.880 & 5.112 & 5.08 & 1.49 \\
\hline 16 & 36 & 0 & 0 & 0 & 0 & 0 \\
\hline 17 & 38 & 0 & 0 & 0 & 0 & 0 \\
\hline 18 & 38 & 2.030 & 3.295 & 5.639 & 3.65 & 1.50 \\
\hline 19 & 40 & 0 & 0 & 0 & 0 & 0 \\
\hline 20 & 40 & 0 & 0 & 0 & 0 & 0 \\
\hline Yield & $45 \%$ & & & & & \\
\hline
\end{tabular}




\begin{tabular}{|c|c|c|c|c|c|c|}
\hline Concentration & $23 \%$ & & & & & \\
\hline Temperature & $100^{\circ} \mathrm{F}$ & & & & & \\
\hline Feed rate & $\begin{array}{c}15 \\
\mathrm{~mm} / \mathrm{s}\end{array}$ & & & & & \\
\hline Needle Gauge & 25 & & & & & \\
\hline Dispense Time & $0.02 \mathrm{~s}$ & & & & & \\
\hline \multicolumn{7}{|l|}{ Short to Long } \\
\hline Fiber \# & $\begin{array}{c}\text { Length } \\
(\mathrm{mm})\end{array}$ & $\begin{array}{c}\text { Leading } \\
\text { Diameter } \\
(\mu \mathrm{m})\end{array}$ & $\begin{array}{c}\text { Middle } \\
\text { Diameter } \\
(\mu \mathrm{m})\end{array}$ & $\begin{array}{c}\text { Terminating } \\
\text { Diameter } \\
(\mu \mathrm{m})\end{array}$ & $\begin{array}{c}\text { Average } \\
\text { Diameter } \\
(\mu \mathrm{m})\end{array}$ & $\begin{array}{c}\text { Standard } \\
\text { Deviation } \\
(\mu \mathrm{m})\end{array}$ \\
\hline 1 & 22 & 0 & 0 & 0 & 0 & 0 \\
\hline 2 & 22 & 0 & 0 & 0 & 0 & 0 \\
\hline 3 & 24 & 4.272 & 5.287 & 10.03 & 6.53 & 2.51 \\
\hline 4 & 24 & 6.417 & 8.828 & 10.27 & 8.51 & 1.59 \\
\hline 5 & 26 & 2.425 & 7.031 & 4.256 & 4.57 & 1.89 \\
\hline 6 & 26 & 5.400 & 4.418 & 10.06 & 6.63 & 2.46 \\
\hline 7 & 28 & 0 & 0 & 0 & 0 & 0 \\
\hline 8 & 28 & 0 & 0 & 0 & 0 & 0 \\
\hline 9 & 30 & 0 & 0 & 0 & 0 & 0 \\
\hline 10 & 30 & 0 & 0 & 0 & 0 & 0 \\
\hline 11 & 32 & 0 & 0 & 0 & 0 & 0 \\
\hline 12 & 32 & 0 & 0 & 0 & 0 & 0 \\
\hline 13 & 34 & 0 & 0 & 0 & 0 & 0 \\
\hline 14 & 34 & 0 & 0 & 0 & 0 & 0 \\
\hline 15 & 36 & 0 & 0 & 0 & 0 & 0 \\
\hline 16 & 36 & 0 & 0 & 0 & 0 & 0 \\
\hline 17 & 38 & 0 & 0 & 0 & 0 & 0 \\
\hline 18 & 38 & 0 & 0 & 0 & 0 & 0 \\
\hline 19 & 40 & 0 & 0 & 0 & 0 & 0 \\
\hline 20 & 40 & 0 & 0 & 0 & 0 & 0 \\
\hline Yield & $20 \%$ & & & & & \\
\hline
\end{tabular}




\begin{tabular}{|c|c|c|c|c|c|c|}
\hline Concentration & $22 \%$ & & & & & \\
\hline Temperature & $70^{\circ} \mathrm{F}$ & & & & & \\
\hline Feed rate & $\begin{array}{c}22.5 \\
\mathrm{~mm} / \mathrm{s}\end{array}$ & & & & & \\
\hline Needle Gauge & 25 & & & & & \\
\hline Dispense Time & $0.02 \mathrm{~s}$ & & & & & \\
\hline \multicolumn{7}{|l|}{ Short to Long } \\
\hline Fiber \# & $\begin{array}{c}\text { Length } \\
(\mathrm{mm})\end{array}$ & $\begin{array}{c}\text { Leading } \\
\text { Diameter } \\
(\mu \mathrm{m})\end{array}$ & $\begin{array}{c}\text { Middle } \\
\text { Diameter } \\
(\mu \mathrm{m})\end{array}$ & $\begin{array}{c}\text { Terminating } \\
\text { Diameter } \\
(\mu \mathrm{m})\end{array}$ & $\begin{array}{c}\text { Average } \\
\text { Diameter } \\
(\mu \mathrm{m})\end{array}$ & $\begin{array}{c}\text { Standard } \\
\text { Deviation } \\
(\mu \mathrm{m})\end{array}$ \\
\hline 1 & 22 & 0 & 0 & 0 & 0.00 & 0.00 \\
\hline 2 & 22 & 2.321 & 1.655 & 3.445 & 2.47 & 0.74 \\
\hline 3 & 24 & 1.074 & 1.261 & 3.631 & 1.99 & 1.16 \\
\hline 4 & 24 & 0.4472 & 2.149 & 2.474 & 1.69 & 0.89 \\
\hline 5 & 26 & 0.6364 & 1.979 & 0.3086 & 0.97 & 0.72 \\
\hline 6 & 26 & 1.757 & 1.893 & 3.543 & 2.40 & 0.81 \\
\hline 7 & 28 & 0.5184 & 2.648 & 3.424 & 2.20 & 1.23 \\
\hline 8 & 28 & 1.590 & 2.088 & 3.703 & 2.46 & 0.90 \\
\hline 9 & 30 & 0.7996 & 0.6476 & 1.058 & 0.84 & 0.17 \\
\hline 10 & 30 & 0.8081 & 0.6086 & 0.8354 & 0.75 & 0.10 \\
\hline 11 & 32 & 0.4479 & 0.5684 & 1.083 & 0.70 & 0.28 \\
\hline 12 & 32 & 0.9318 & 0.3641 & 0.9729 & 0.76 & 0.28 \\
\hline 13 & 34 & 0 & 0 & 0 & 0.00 & 0.00 \\
\hline 14 & 34 & 0 & 0 & 0 & 0.00 & 0.00 \\
\hline 15 & 36 & 0 & 0 & 0 & 0.00 & 0.00 \\
\hline 16 & 36 & 0 & 0 & 0 & 0.00 & 0.00 \\
\hline 17 & 38 & 0 & 0 & 0 & 0.00 & 0.00 \\
\hline 18 & 38 & 0 & 0 & 0 & 0.00 & 0.00 \\
\hline 19 & 40 & 0 & 0 & 0 & 0.00 & 0.00 \\
\hline 20 & 40 & 0 & 0 & 0 & 0.00 & 0.00 \\
\hline Yield & $55 \%$ & & & & & \\
\hline
\end{tabular}




\begin{tabular}{|c|c|c|c|c|c|c|}
\hline Concentration & $22 \%$ & & & & & \\
\hline Temperature & $70^{\circ} \mathrm{F}$ & & & & & \\
\hline Feed rate & $\begin{array}{c}15 \\
\mathrm{~mm} / \mathrm{s}\end{array}$ & & & & & \\
\hline Needle Gauge & 25 & & & & & \\
\hline Dispense Time & $0.02 \mathrm{~s}$ & & & & & \\
\hline \multicolumn{7}{|l|}{ Short to Long } \\
\hline Fiber \# & $\begin{array}{c}\text { Length } \\
(\mathrm{mm})\end{array}$ & $\begin{array}{c}\text { Leading } \\
\text { Diameter } \\
(\mu \mathrm{m})\end{array}$ & $\begin{array}{c}\text { Middle } \\
\text { Diameter } \\
(\mu \mathrm{m})\end{array}$ & $\begin{array}{c}\text { Terminating } \\
\text { Diameter } \\
(\mu \mathrm{m})\end{array}$ & $\begin{array}{c}\text { Average } \\
\text { Diameter } \\
(\mu \mathrm{m})\end{array}$ & $\begin{array}{c}\text { Standard } \\
\text { Deviation } \\
(\mu \mathrm{m})\end{array}$ \\
\hline 1 & 22 & 0 & 0 & 0 & 0.00 & 0.00 \\
\hline 2 & 22 & 0.6379 & 1.494 & 1.719 & 1.28 & 0.47 \\
\hline 3 & 24 & 0.6726 & 0.6925 & 0.9669 & 0.78 & 0.13 \\
\hline 4 & 24 & 1.271 & 1.718 & 1.951 & 1.65 & 0.28 \\
\hline 5 & 26 & 1.690 & 1.071 & 1.787 & 1.52 & 0.32 \\
\hline 6 & 26 & 1.115 & 1.730 & 1.428 & 1.42 & 0.25 \\
\hline 7 & 28 & 2.225 & 2.601 & 2.142 & 2.32 & 0.20 \\
\hline 8 & 28 & 0.389 & 1.122 & 2.349 & 1.29 & 0.81 \\
\hline 9 & 30 & 0 & 0 & 0 & 0.00 & 0.00 \\
\hline 10 & 30 & 0 & 0 & 0 & 0.00 & 0.00 \\
\hline 11 & 32 & 0 & 0 & 0 & 0.00 & 0.00 \\
\hline 12 & 32 & 0 & 0 & 0 & 0.00 & 0.00 \\
\hline 13 & 34 & 0 & 0 & 0 & 0.00 & 0.00 \\
\hline 14 & 34 & 1.353 & 1.9 & 1.137 & 1.46 & 0.32 \\
\hline 15 & 36 & 0 & 0 & 0 & 0.00 & 0.00 \\
\hline 16 & 36 & 0 & 0 & 0 & 0.00 & 0.00 \\
\hline 17 & 38 & 0 & 0 & 0 & 0.00 & 0.00 \\
\hline 18 & 38 & 0 & 0 & 0 & 0.00 & 0.00 \\
\hline 19 & 40 & 0 & 0 & 0 & 0.00 & 0.00 \\
\hline 20 & 40 & 0 & 0 & 0 & 0.00 & 0.00 \\
\hline Yield & $40 \%$ & & & & & \\
\hline
\end{tabular}




\begin{tabular}{|c|c|c|c|c|c|c|}
\hline Concentration & $22 \%$ & & & & & \\
\hline Temperature & $90^{\circ} \mathrm{F}$ & & & & & \\
\hline Feed rate & $\begin{array}{c}22.5 \\
\mathrm{~mm} / \mathrm{s}\end{array}$ & & & & & \\
\hline Needle Gauge & 25 & & & & & \\
\hline Dispense Time & $0.02 \mathrm{~s}$ & & & & & \\
\hline \multicolumn{7}{|l|}{ Short to Long } \\
\hline Fiber \# & $\begin{array}{c}\text { Length } \\
(\mathrm{mm})\end{array}$ & $\begin{array}{c}\text { Leading } \\
\text { Diameter } \\
(\mu \mathrm{m})\end{array}$ & $\begin{array}{c}\text { Middle } \\
\text { Diameter } \\
(\mu \mathrm{m})\end{array}$ & $\begin{array}{c}\text { Terminating } \\
\text { Diameter } \\
(\mu \mathrm{m})\end{array}$ & $\begin{array}{c}\text { Average } \\
\text { Diameter } \\
(\mu \mathrm{m})\end{array}$ & $\begin{array}{c}\text { Standard } \\
\text { Deviation } \\
(\mu \mathrm{m})\end{array}$ \\
\hline 1 & 22 & 0 & 0 & 0 & 0 & 0 \\
\hline 2 & 22 & 5.912 & 8.910 & 6.185 & 7.00 & 1.35 \\
\hline 3 & 24 & 2.970 & 6.226 & 11.49 & 6.90 & 3.51 \\
\hline 4 & 24 & 10.03 & 16.75 & 6.406 & 11.06 & 4.29 \\
\hline 5 & 26 & 3.669 & 4.333 & 6.893 & 4.97 & 1.39 \\
\hline 6 & 26 & 5.190 & 7.021 & 6.854 & 6.36 & 0.83 \\
\hline 7 & 28 & 6.504 & 5.038 & 5.360 & 5.63 & 0.63 \\
\hline 8 & 28 & 2.199 & 12.64 & 4.067 & 6.30 & 4.55 \\
\hline 9 & 30 & 2.992 & 7.479 & 3.943 & 4.80 & 1.93 \\
\hline 10 & 30 & 3.616 & 2.937 & 3.505 & 3.35 & 0.30 \\
\hline 11 & 32 & 9.059 & 2.950 & 3.078 & 5.03 & 2.85 \\
\hline 12 & 32 & 9.829 & 5.953 & 5.905 & 7.23 & 1.84 \\
\hline 13 & 34 & 6.540 & 3.540 & 3.547 & 4.54 & 1.41 \\
\hline 14 & 34 & 0 & 0 & 0 & 0.00 & 0.00 \\
\hline 15 & 36 & 0 & 0 & 0 & 0.00 & 0.00 \\
\hline 16 & 36 & 8.508 & 6.493 & 6.958 & 7.32 & 0.86 \\
\hline 17 & 38 & 0 & 0 & 0 & 0 & 0 \\
\hline 18 & 38 & 0 & 0 & 0 & 0 & 0 \\
\hline 19 & 40 & 0 & 0 & 0 & 0 & 0 \\
\hline 20 & 40 & 0 & 0 & 0 & 0 & 0 \\
\hline Yield & $65 \%$ & & & & & \\
\hline
\end{tabular}




\begin{tabular}{|c|c|c|c|c|c|c|}
\hline Concentration & $22 \%$ & & & & & \\
\hline Temperature & $90^{\circ} \mathrm{F}$ & & & & & \\
\hline Feed rate & $\begin{array}{c}15 \\
\mathrm{~mm} / \mathrm{s}\end{array}$ & & & & & \\
\hline Needle Gauge & 25 & & & & & \\
\hline Dispense Time & $0.02 \mathrm{~s}$ & & & & & \\
\hline \multicolumn{7}{|l|}{ Short to Long } \\
\hline Fiber \# & $\begin{array}{c}\text { Length } \\
(\mathrm{mm})\end{array}$ & $\begin{array}{c}\text { Leading } \\
\text { Diameter } \\
(\mu \mathrm{m})\end{array}$ & $\begin{array}{c}\text { Middle } \\
\text { Diameter } \\
(\mu \mathrm{m})\end{array}$ & $\begin{array}{c}\text { Terminating } \\
\text { Diameter } \\
(\mu \mathrm{m})\end{array}$ & $\begin{array}{c}\text { Average } \\
\text { Diameter } \\
(\mu \mathrm{m})\end{array}$ & $\begin{array}{c}\text { Standard } \\
\text { Deviation } \\
(\mu \mathrm{m})\end{array}$ \\
\hline 1 & 22 & 0 & 0 & 0 & 0 & 0 \\
\hline 2 & 22 & 5.773 & 2.428 & 4.795 & 4.33 & 1.40 \\
\hline 3 & 24 & 0 & 0 & 0 & 0 & 0 \\
\hline 4 & 24 & 4.014 & 4.944 & 3.087 & 4.02 & 0.76 \\
\hline 5 & 26 & 2.892 & 7.265 & 6.877 & 5.68 & 1.98 \\
\hline 6 & 26 & 0 & 0 & 0 & 0 & 0 \\
\hline 7 & 28 & 0 & 0 & 0 & 0 & 0 \\
\hline 8 & 28 & 0 & 0 & 0 & 0 & 0 \\
\hline 9 & 30 & 0 & 0 & 0 & 0 & 0 \\
\hline 10 & 30 & 0 & 0 & 0 & 0 & 0 \\
\hline 11 & 32 & 0 & 0 & 0 & 0 & 0 \\
\hline 12 & 32 & 0 & 0 & 0 & 0 & 0 \\
\hline 13 & 34 & 0 & 0 & 0 & 0 & 0 \\
\hline 14 & 34 & 0 & 0 & 0 & 0 & 0 \\
\hline 15 & 36 & 0 & 0 & 0 & 0 & 0 \\
\hline 16 & 36 & 0 & 0 & 0 & 0 & 0 \\
\hline 17 & 38 & 0 & 0 & 0 & 0 & 0 \\
\hline 18 & 38 & 0 & 0 & 0 & 0 & 0 \\
\hline 19 & 40 & 2.350 & 7.723 & 6.872 & 5.65 & 2.36 \\
\hline 20 & 40 & 8.655 & 8.440 & 7.847 & 8.31 & 0.34 \\
\hline Yield & $25 \%$ & & & & & \\
\hline
\end{tabular}




\begin{tabular}{|c|c|c|c|c|c|c|}
\hline Concentration & $22 \%$ & & & & & \\
\hline Temperature & $100^{\circ} \mathrm{F}$ & & & & & \\
\hline Feed rate & $\begin{array}{c}22.5 \\
\mathrm{~mm} / \mathrm{s}\end{array}$ & & & & & \\
\hline Needle Gauge & 25 & & & & & \\
\hline Dispense Time & $0.02 \mathrm{~s}$ & & & & & \\
\hline \multicolumn{7}{|l|}{ Short to Long } \\
\hline Fiber \# & $\begin{array}{c}\text { Length } \\
(\mathrm{mm})\end{array}$ & $\begin{array}{c}\text { Leading } \\
\text { Diameter } \\
(\mu \mathrm{m})\end{array}$ & $\begin{array}{c}\text { Middle } \\
\text { Diameter } \\
(\mu \mathrm{m})\end{array}$ & $\begin{array}{c}\text { Terminating } \\
\text { Diameter } \\
(\mu \mathrm{m})\end{array}$ & $\begin{array}{c}\text { Average } \\
\text { Diameter } \\
(\mu \mathrm{m})\end{array}$ & $\begin{array}{c}\text { Standard } \\
\text { Deviation } \\
(\mu \mathrm{m})\end{array}$ \\
\hline 1 & 22 & 0 & 0 & 0 & 0 & 0 \\
\hline 2 & 22 & 68.3 & 24.52 & 40.77 & 44.53 & 18.07 \\
\hline 3 & 24 & 6.009 & 2.895 & 14.94 & 7.95 & 5.10 \\
\hline 4 & 24 & 2.727 & 8.275 & 7.151 & 6.05 & 2.39 \\
\hline 5 & 26 & 3.516 & 4.669 & 4.280 & 4.16 & 0.48 \\
\hline 6 & 26 & 5.313 & 3.999 & 4.123 & 4.48 & 0.59 \\
\hline 7 & 28 & 0 & 0 & 0 & 0.00 & 0.00 \\
\hline 8 & 28 & 7.452 & 11.44 & 10.18 & 9.69 & 1.66 \\
\hline 9 & 30 & 9.975 & 9.034 & 10.01 & 9.67 & 0.45 \\
\hline 10 & 30 & 12.59 & 2.988 & 3.115 & 6.23 & 4.50 \\
\hline 11 & 32 & 8.401 & 11.73 & 13.85 & 11.33 & 2.24 \\
\hline 12 & 32 & 0 & 0 & 0 & 0.00 & 0.00 \\
\hline 13 & 34 & 0 & 0 & 0 & 0.00 & 0.00 \\
\hline 14 & 34 & 10.63 & 3.591 & 9.153 & 7.79 & 3.03 \\
\hline 15 & 36 & 5.115 & 8.095 & 32.07 & 15.09 & 12.07 \\
\hline 16 & 36 & 11.06 & 8.850 & 18.66 & 12.86 & 4.20 \\
\hline 17 & 38 & 0 & 0 & 0 & 0.00 & 0.00 \\
\hline 18 & 38 & 6.611 & 9.936 & 4.261 & 6.94 & 2.33 \\
\hline 19 & 40 & 0 & 0 & 0 & 0.00 & 0.00 \\
\hline 20 & 40 & 13.89 & 7.612 & 13.89 & 11.80 & 2.96 \\
\hline Yield & $70 \%$ & & & & & \\
\hline
\end{tabular}




\begin{tabular}{|c|c|c|c|c|c|c|}
\hline Concentration & $22 \%$ & & & & & \\
\hline Temperature & $100^{\circ} \mathrm{F}$ & & & & & \\
\hline Feed rate & $\begin{array}{c}15 \\
\mathrm{~mm} / \mathrm{s}\end{array}$ & & & & & \\
\hline Needle Gauge & 25 & & & & & \\
\hline Dispense Time & $0.02 \mathrm{~s}$ & & & & & \\
\hline \multicolumn{7}{|l|}{ Short to Long } \\
\hline Fiber \# & $\begin{array}{c}\text { Length } \\
(\mathrm{mm})\end{array}$ & $\begin{array}{c}\text { Leading } \\
\text { Diameter } \\
(\mu \mathrm{m})\end{array}$ & $\begin{array}{c}\text { Middle } \\
\text { Diameter } \\
(\mu \mathrm{m})\end{array}$ & $\begin{array}{c}\text { Terminating } \\
\text { Diameter } \\
(\mu \mathrm{m})\end{array}$ & $\begin{array}{c}\text { Average } \\
\text { Diameter } \\
(\mu \mathrm{m})\end{array}$ & $\begin{array}{c}\text { Standard } \\
\text { Deviation } \\
(\mu \mathrm{m})\end{array}$ \\
\hline 1 & 22 & 0 & 0 & 0 & 0 & 0 \\
\hline 2 & 22 & 0 & 0 & 0 & 0 & 0 \\
\hline 3 & 24 & 0 & 0 & 0 & 0 & 0 \\
\hline 4 & 24 & 7.541 & 12.10 & 21.50 & 13.71 & 5.81 \\
\hline 5 & 26 & 6.394 & 6.631 & 12.92 & 8.65 & 3.02 \\
\hline 6 & 26 & 8.817 & 17.78 & 13.98 & 13.53 & 3.67 \\
\hline 7 & 28 & 0 & 0 & 0 & 0 & 0 \\
\hline 8 & 28 & 9.328 & 11.52 & 24.68 & 15.18 & 6.78 \\
\hline 9 & 30 & 6.832 & 10.16 & 15.24 & 10.74 & 3.46 \\
\hline 10 & 30 & 5.985 & 11.01 & 14.49 & 10.50 & 3.49 \\
\hline 11 & 32 & 9.587 & 7.759 & 11.96 & 9.77 & 1.72 \\
\hline 12 & 32 & 0 & 0 & 0 & & \\
\hline 13 & 34 & 0 & 0 & 0 & 0 & 0 \\
\hline 14 & 34 & 0 & 0 & 0 & 0 & 0 \\
\hline 15 & 36 & 0 & 0 & 0 & 0 & 0 \\
\hline 16 & 36 & 0 & 0 & 0 & 0 & 0 \\
\hline 17 & 38 & 0 & 0 & 0 & 0 & 0 \\
\hline 18 & 38 & 0 & 0 & 0 & 0 & 0 \\
\hline 19 & 40 & 0 & 0 & 0 & 0 & 0 \\
\hline 20 & 40 & 0 & 0 & 0 & 0 & 0 \\
\hline Yield & $35 \%$ & & & & & \\
\hline
\end{tabular}




\begin{tabular}{|c|c|c|c|c|c|c|}
\hline Concentration & $22 \%$ & & & & & \\
\hline Temperature & $80^{\circ} \mathrm{F}$ & & & & & \\
\hline Feed rate & $\begin{array}{c}15 \\
\mathrm{~mm} / \mathrm{s}\end{array}$ & & & & & \\
\hline Needle Gauge & 25 & & & & & \\
\hline Dispense Time & $0.02 \mathrm{~s}$ & & & & & \\
\hline \multicolumn{7}{|l|}{ Short to Long } \\
\hline Fiber \# & $\begin{array}{c}\text { Length } \\
(\mathrm{mm})\end{array}$ & $\begin{array}{c}\text { Leading } \\
\text { Diameter } \\
(\mu \mathrm{m})\end{array}$ & $\begin{array}{c}\text { Middle } \\
\text { Diameter } \\
(\mu \mathrm{m})\end{array}$ & $\begin{array}{c}\text { Terminating } \\
\text { Diameter } \\
(\mu \mathrm{m})\end{array}$ & $\begin{array}{c}\text { Average } \\
\text { Diameter } \\
(\mu \mathrm{m})\end{array}$ & $\begin{array}{c}\text { Standard } \\
\text { Deviation } \\
(\mu \mathrm{m})\end{array}$ \\
\hline 1 & 22 & 0 & 0 & 0 & 0 & 0 \\
\hline 2 & 22 & 21.34 & 10.84 & 17.38 & 16.52 & 4.33 \\
\hline 3 & 24 & 18.51 & 9.221 & 5.778 & 11.17 & 5.38 \\
\hline 4 & 24 & 10.84 & 13.11 & 9.355 & 11.10 & 1.54 \\
\hline 5 & 26 & 0 & 0 & 0 & 0 & 0 \\
\hline 6 & 26 & 10.39 & 6.821 & 8.127 & 8.45 & 1.47 \\
\hline 7 & 28 & 7.566 & 12.52 & 10.30 & 10.13 & 2.03 \\
\hline 8 & 28 & 20.60 & 14.92 & 7.405 & 14.31 & 5.40 \\
\hline 9 & 30 & 6.817 & 7.662 & 9.465 & 7.98 & 1.10 \\
\hline 10 & 30 & 0 & 0 & 0 & 0 & 0 \\
\hline 11 & 32 & 0 & 0 & 0 & 0 & 0 \\
\hline 12 & 32 & 0 & 0 & 0 & 0 & 0 \\
\hline 13 & 34 & 0 & 0 & 0 & 0 & 0 \\
\hline 14 & 34 & 41.91 & 27.09 & 16.56 & 28.52 & 10.40 \\
\hline 15 & 36 & 6.703 & 5.169 & 11.99 & 7.95 & 2.92 \\
\hline 16 & 36 & 4.233 & 8.596 & 11.27 & 8.03 & 2.90 \\
\hline 17 & 38 & 12.70 & 12.26 & 9.477 & 11.48 & 1.43 \\
\hline 18 & 38 & 7.990 & 5.347 & 3.963 & 5.77 & 1.67 \\
\hline 19 & 40 & 0 & 0 & 0 & 0 & 0 \\
\hline 20 & 40 & 0 & 0 & 0 & 0 & 0 \\
\hline Yield & $60 \%$ & & & & & \\
\hline
\end{tabular}




\begin{tabular}{|c|c|c|c|c|c|c|}
\hline Concentration & $22 \%$ & & & & & \\
\hline Temperature & $80^{\circ} \mathrm{F}$ & & & & & \\
\hline Feed rate & $\begin{array}{c}22.5 \\
\mathrm{~mm} / \mathrm{s}\end{array}$ & & & & & \\
\hline Needle Gauge & 25 & & & & & \\
\hline Dispense Time & $0.02 \mathrm{~s}$ & & & & & \\
\hline \multicolumn{7}{|l|}{ Short to Long } \\
\hline Fiber \# & $\begin{array}{c}\text { Length } \\
(\mathrm{mm})\end{array}$ & $\begin{array}{c}\text { Leading } \\
\text { Diameter } \\
(\mu \mathrm{m})\end{array}$ & $\begin{array}{c}\text { Middle } \\
\text { Diameter } \\
(\mu \mathrm{m})\end{array}$ & $\begin{array}{c}\text { Terminating } \\
\text { Diameter } \\
(\mu \mathrm{m})\end{array}$ & $\begin{array}{c}\text { Average } \\
\text { Diameter } \\
(\mu \mathrm{m})\end{array}$ & $\begin{array}{c}\text { Standard } \\
\text { Deviation } \\
(\mu \mathrm{m})\end{array}$ \\
\hline 1 & 22 & 0 & 0 & 0 & 0 & 0 \\
\hline 2 & 22 & 6.528 & 7.590 & 15.67 & 9.93 & 4.08 \\
\hline 3 & 24 & 0 & 0 & 0 & 0 & 0 \\
\hline 4 & 24 & 9.696 & 17.58 & 8.717 & 12.00 & 3.97 \\
\hline 5 & 26 & 5.737 & 12.10 & 12.39 & 10.08 & 3.07 \\
\hline 6 & 26 & 6.840 & 5.512 & 7.483 & 6.61 & 0.82 \\
\hline 7 & 28 & 6.834 & 4.565 & 3.863 & 5.09 & 1.27 \\
\hline 8 & 28 & 0 & 0 & 0 & 0 & 0 \\
\hline 9 & 30 & 7.577 & 10.72 & 5.222 & 7.84 & 2.25 \\
\hline 10 & 30 & 0 & 0 & 0 & 0 & 0 \\
\hline 11 & 32 & 5.634 & 2.420 & 9.248 & 5.77 & 2.79 \\
\hline 12 & 32 & 0 & 0 & 0 & 0 & 0 \\
\hline 13 & 34 & 0 & 0 & 0 & 0 & 0 \\
\hline 14 & 34 & 0 & 0 & 0 & 0 & 0 \\
\hline 15 & 36 & 0 & 0 & 0 & 0 & 0 \\
\hline 16 & 36 & 0 & 0 & 0 & 0 & 0 \\
\hline 17 & 38 & 0 & 0 & 0 & 0 & 0 \\
\hline 18 & 38 & 0 & 0 & 0 & 0 & 0 \\
\hline 19 & 40 & 0 & 0 & 0 & 0 & 0 \\
\hline 20 & 40 & 0 & 0 & 0 & 0 & 0 \\
\hline Yield & $35 \%$ & & & & & \\
\hline
\end{tabular}




\begin{tabular}{|c|c|c|c|c|c|c|}
\hline Concentration & $21 \%$ & & & & & \\
\hline Temperature & $100^{\circ} \mathrm{F}$ & & & & & \\
\hline Feed rate & $\begin{array}{c}22.5 \\
\mathrm{~mm} / \mathrm{s}\end{array}$ & & & & & \\
\hline Needle Gauge & 25 & & & & & \\
\hline Dispense Time & $0.02 \mathrm{~s}$ & & & & & \\
\hline \multicolumn{7}{|l|}{ Short to Long } \\
\hline Fiber \# & $\begin{array}{c}\text { Length } \\
(\mathrm{mm})\end{array}$ & $\begin{array}{c}\text { Leading } \\
\text { Diameter } \\
(\mu \mathrm{m})\end{array}$ & $\begin{array}{c}\text { Middle } \\
\text { Diameter } \\
(\mu \mathrm{m})\end{array}$ & $\begin{array}{c}\text { Terminating } \\
\text { Diameter } \\
(\mu \mathrm{m})\end{array}$ & $\begin{array}{c}\text { Average } \\
\text { Diameter } \\
(\mu \mathrm{m})\end{array}$ & $\begin{array}{c}\text { Standard } \\
\text { Deviation } \\
(\mu \mathrm{m})\end{array}$ \\
\hline 1 & 22 & 0 & 0 & 0 & 0 & 0 \\
\hline 2 & 22 & 2.469 & 2.666 & 2.897 & 2.68 & 0.17 \\
\hline 3 & 24 & 2.851 & 6.176 & 4.282 & 4.44 & 1.36 \\
\hline 4 & 24 & 2.312 & 2.437 & 3.317 & 2.69 & 0.45 \\
\hline 5 & 26 & 0 & 0 & 0 & 0 & 0 \\
\hline 6 & 26 & 0 & 0 & 0 & 0 & 0 \\
\hline 7 & 28 & 0 & 0 & 0 & 0 & 0 \\
\hline 8 & 28 & 0 & 0 & 0 & 0 & 0 \\
\hline 9 & 30 & 0 & 0 & 0 & 0 & 0 \\
\hline 10 & 30 & 0 & 0 & 0 & 0 & 0 \\
\hline 11 & 32 & 0 & 0 & 0 & 0 & 0 \\
\hline 12 & 32 & 0 & 0 & 0 & 0 & 0 \\
\hline 13 & 34 & 0 & 0 & 0 & 0 & 0 \\
\hline 14 & 34 & 0 & 0 & 0 & 0 & 0 \\
\hline 15 & 36 & 0 & 0 & 0 & 0 & 0 \\
\hline 16 & 36 & 0 & 0 & 0 & 0 & 0 \\
\hline 17 & 38 & 0 & 0 & 0 & 0 & 0 \\
\hline 18 & 38 & 0 & 0 & 0 & 0 & 0 \\
\hline 19 & 40 & 0 & 0 & 0 & 0 & 0 \\
\hline 20 & 40 & 0 & 0 & 0 & 0 & 0 \\
\hline Yield & $15 \%$ & & & & & \\
\hline
\end{tabular}




\begin{tabular}{|c|c|c|c|c|c|c|}
\hline Concentration & $21 \%$ & & & & & \\
\hline Temperature & $100^{\circ} \mathrm{F}$ & & & & & \\
\hline Feed rate & $\begin{array}{c}15 \\
\mathrm{~mm} / \mathrm{s}\end{array}$ & & & & & \\
\hline Needle Gauge & 25 & & & & & \\
\hline Dispense Time & $0.02 \mathrm{~s}$ & & & & & \\
\hline \multicolumn{7}{|l|}{ Short to Long } \\
\hline Fiber \# & $\begin{array}{c}\text { Length } \\
(\mathrm{mm})\end{array}$ & $\begin{array}{c}\text { Leading } \\
\text { Diameter } \\
(\mu \mathrm{m})\end{array}$ & $\begin{array}{c}\text { Middle } \\
\text { Diameter } \\
(\mu \mathrm{m})\end{array}$ & $\begin{array}{c}\text { Terminating } \\
\text { Diameter } \\
(\mu \mathrm{m})\end{array}$ & $\begin{array}{c}\text { Average } \\
\text { Diameter } \\
(\mu \mathrm{m})\end{array}$ & $\begin{array}{c}\text { Standard } \\
\text { Deviation } \\
(\mu \mathrm{m})\end{array}$ \\
\hline 1 & 22 & 0 & 0 & 0 & 0 & 0 \\
\hline 2 & 22 & 0 & 0 & 0 & 0 & 0 \\
\hline 3 & 24 & 0 & 0 & 0 & 0 & 0 \\
\hline 4 & 24 & 0 & 0 & 0 & 0 & 0 \\
\hline 5 & 26 & 0 & 0 & 0 & 0 & 0 \\
\hline 6 & 26 & 5.503 & 5.066 & 0.9633 & 3.84 & 2.04 \\
\hline 7 & 28 & 6.274 & 1.097 & 2.540 & 3.30 & 2.18 \\
\hline 8 & 28 & 1.720 & 3.928 & 0.9895 & 2.21 & 1.25 \\
\hline 9 & 30 & 0 & 0 & 0 & 0 & 0 \\
\hline 10 & 30 & 0 & 0 & 0 & 0 & 0 \\
\hline 11 & 32 & 0 & 0 & 0 & 0 & 0 \\
\hline 12 & 32 & 0 & 0 & 0 & 0 & 0 \\
\hline 13 & 34 & 0 & 0 & 0 & 0 & 0 \\
\hline 14 & 34 & 0 & 0 & 0 & 0 & 0 \\
\hline 15 & 36 & 0 & 0 & 0 & 0 & 0 \\
\hline 16 & 36 & 0 & 0 & 0 & 0 & 0 \\
\hline 17 & 38 & 0 & 0 & 0 & 0 & 0 \\
\hline 18 & 38 & 0 & 0 & 0 & 0 & 0 \\
\hline 19 & 40 & 0 & 0 & 0 & 0 & 0 \\
\hline 20 & 40 & 0 & 0 & 0 & 0 & 0 \\
\hline Yield & $15 \%$ & & & & & \\
\hline
\end{tabular}




\begin{tabular}{|c|c|c|c|c|c|c|}
\hline Concentration & $21 \%$ & & & & & \\
\hline Temperature & $90^{\circ} \mathrm{F}$ & & & & & \\
\hline Feed rate & $\begin{array}{c}15 \\
\mathrm{~mm} / \mathrm{s}\end{array}$ & & & & & \\
\hline Needle Gauge & 25 & & & & & \\
\hline Dispense Time & $0.02 \mathrm{~s}$ & & & & & \\
\hline \multicolumn{7}{|l|}{ Short to Long } \\
\hline Fiber \# & $\begin{array}{c}\text { Length } \\
(\mathrm{mm})\end{array}$ & $\begin{array}{c}\text { Leading } \\
\text { Diameter } \\
(\mu \mathrm{m})\end{array}$ & $\begin{array}{c}\text { Middle } \\
\text { Diameter } \\
(\mu \mathrm{m})\end{array}$ & $\begin{array}{c}\text { Terminating } \\
\text { Diameter } \\
(\mu \mathrm{m})\end{array}$ & $\begin{array}{c}\text { Average } \\
\text { Diameter } \\
(\mu \mathrm{m})\end{array}$ & $\begin{array}{c}\text { Standard } \\
\text { Deviation } \\
(\mu \mathrm{m})\end{array}$ \\
\hline 1 & 22 & 0 & 0 & 0 & 0 & 0 \\
\hline 2 & 22 & 1.214 & 2.526 & 3.041 & 2.26 & 0.77 \\
\hline 3 & 24 & 2.948 & 5.769 & 2.735 & 3.82 & 1.38 \\
\hline 4 & 24 & 0 & 0 & 0 & 0.00 & 0.00 \\
\hline 5 & 26 & 3.732 & 7.453 & 5.418 & 5.53 & 1.52 \\
\hline 6 & 26 & 0 & 0 & 0 & 0 & 0 \\
\hline 7 & 28 & 0 & 0 & 0 & 0 & 0 \\
\hline 8 & 28 & 1.293 & 5.434 & 7.408 & 4.71 & 2.55 \\
\hline 9 & 30 & 5.066 & 2.381 & 5.442 & 4.30 & 1.36 \\
\hline 10 & 30 & 2.244 & 6.657 & 7.366 & 5.42 & 2.27 \\
\hline 11 & 32 & 1.078 & 4.264 & 3.650 & 3.00 & 1.38 \\
\hline 12 & 32 & 0 & 0 & 0 & 0 & 0 \\
\hline 13 & 34 & 0.8269 & 5.087 & 6.008 & 3.97 & 2.26 \\
\hline 14 & 34 & 0 & 0 & 0 & 0 & 0 \\
\hline 15 & 36 & 0 & 0 & 0 & 0 & 0 \\
\hline 16 & 36 & 0 & 0 & 0 & 0 & 0 \\
\hline 17 & 38 & 0 & 0 & 0 & 0 & 0 \\
\hline 18 & 38 & 1.569 & 2.751 & 7.266 & 3.86 & 2.45 \\
\hline 19 & 40 & 0 & 0 & 0 & 0 & 0 \\
\hline 20 & 40 & 0 & 0 & 0 & 0 & 0 \\
\hline Yield & $45 \%$ & & & & & \\
\hline
\end{tabular}




\begin{tabular}{|c|c|c|c|c|c|c|}
\hline Concentration & $21 \%$ & & & & & \\
\hline Temperature & $90^{\circ} \mathrm{F}$ & & & & & \\
\hline Feed rate & $\begin{array}{c}22.5 \\
\mathrm{~mm} / \mathrm{s}\end{array}$ & & & & & \\
\hline Needle Gauge & 25 & & & & & \\
\hline Dispense Time & $0.02 \mathrm{~s}$ & & & & & \\
\hline \multicolumn{7}{|l|}{ Short to Long } \\
\hline Fiber \# & $\begin{array}{c}\text { Length } \\
(\mathrm{mm})\end{array}$ & $\begin{array}{c}\text { Leading } \\
\text { Diameter } \\
(\mu \mathrm{m})\end{array}$ & $\begin{array}{c}\text { Middle } \\
\text { Diameter } \\
(\mu \mathrm{m})\end{array}$ & $\begin{array}{c}\text { Terminating } \\
\text { Diameter } \\
(\mu \mathrm{m})\end{array}$ & $\begin{array}{c}\text { Average } \\
\text { Diameter } \\
(\mu \mathrm{m})\end{array}$ & $\begin{array}{c}\text { Standard } \\
\text { Deviation } \\
(\mu \mathrm{m})\end{array}$ \\
\hline 1 & 22 & 0 & 0 & 0 & 0 & 0 \\
\hline 2 & 22 & 0 & 0 & 0 & 0 & 0 \\
\hline 3 & 24 & 1.589 & 0.8731 & 2.130 & 1.53 & 0.51 \\
\hline 4 & 24 & 2.328 & 3.989 & 1.831 & 2.72 & 0.92 \\
\hline 5 & 26 & 6.574 & 2.142 & 5.216 & 4.64 & 1.85 \\
\hline 6 & 26 & 2.318 & 5.077 & 6.878 & 4.76 & 1.88 \\
\hline 7 & 28 & 0 & 0 & 0 & 0 & 0 \\
\hline 8 & 28 & 0 & 0 & 0 & 0 & 0 \\
\hline 9 & 30 & 0 & 0 & 0 & 0 & 0 \\
\hline 10 & 30 & 0 & 0 & 0 & 0 & 0 \\
\hline 11 & 32 & 0 & 0 & 0 & 0 & 0 \\
\hline 12 & 32 & 0 & 0 & 0 & 0 & 0 \\
\hline 13 & 34 & 0 & 0 & 0 & 0 & 0 \\
\hline 14 & 34 & 0 & 0 & 0 & 0 & 0 \\
\hline 15 & 36 & 0 & 0 & 0 & 0 & 0 \\
\hline 16 & 36 & 0 & 0 & 0 & 0 & 0 \\
\hline 17 & 38 & 0 & 0 & 0 & 0 & 0 \\
\hline 18 & 38 & 0 & 0 & 0 & 0 & 0 \\
\hline 19 & 40 & 0 & 0 & 0 & 0 & 0 \\
\hline 20 & 40 & 0 & 0 & 0 & 0 & 0 \\
\hline Yield & $20 \%$ & & & & & \\
\hline
\end{tabular}




\begin{tabular}{|c|c|c|c|c|c|c|}
\hline Concentration & $21 \%$ & & & & & \\
\hline Temperature & $80^{\circ} \mathrm{F}$ & & & & & \\
\hline Feed rate & $\begin{array}{c}22.5 \\
\mathrm{~mm} / \mathrm{s}\end{array}$ & & & & & \\
\hline Needle Gauge & 25 & & & & & \\
\hline Dispense Time & $0.02 \mathrm{~s}$ & & & & & \\
\hline \multicolumn{7}{|l|}{ Short to Long } \\
\hline Fiber \# & $\begin{array}{c}\text { Length } \\
(\mathrm{mm})\end{array}$ & $\begin{array}{c}\text { Leading } \\
\text { Diameter } \\
(\mu \mathrm{m})\end{array}$ & $\begin{array}{c}\text { Middle } \\
\text { Diameter } \\
(\mu \mathrm{m})\end{array}$ & $\begin{array}{c}\text { Terminating } \\
\text { Diameter } \\
(\mu \mathrm{m})\end{array}$ & $\begin{array}{c}\text { Average } \\
\text { Diameter } \\
(\mu \mathrm{m})\end{array}$ & $\begin{array}{c}\text { Standard } \\
\text { Deviation } \\
(\mu \mathrm{m})\end{array}$ \\
\hline 1 & 22 & 0 & 0 & 0 & 0 & 0 \\
\hline 2 & 22 & 0 & 0 & 0 & 0 & 0 \\
\hline 3 & 24 & 0 & 0 & 0 & 0 & 0 \\
\hline 4 & 24 & 1.472 & 11.31 & 3.113 & 5.30 & 4.30 \\
\hline 5 & 26 & 0 & 0 & 0 & 0 & 0 \\
\hline 6 & 26 & 0 & 0 & 0 & 0 & 0 \\
\hline 7 & 28 & 0 & 0 & 0 & 0 & 0 \\
\hline 8 & 28 & 0 & 0 & 0 & 0 & 0 \\
\hline 9 & 30 & 0 & 0 & 0 & 0 & 0 \\
\hline 10 & 30 & 0 & 0 & 0 & 0 & 0 \\
\hline 11 & 32 & 0 & 0 & 0 & 0 & 0 \\
\hline 12 & 32 & 0 & 0 & 0 & 0 & 0 \\
\hline 13 & 34 & 0 & 0 & 0 & 0 & 0 \\
\hline 14 & 34 & 0 & 0 & 0 & 0 & 0 \\
\hline 15 & 36 & 0 & 0 & 0 & 0 & 0 \\
\hline 16 & 36 & 0 & 0 & 0 & 0 & 0 \\
\hline 17 & 38 & 0 & 0 & 0 & 0 & 0 \\
\hline 18 & 38 & 0 & 0 & 0 & 0 & 0 \\
\hline 19 & 40 & 0 & 0 & 0 & 0 & 0 \\
\hline 20 & 40 & 0 & 0 & 0 & 0 & 0 \\
\hline Yield & $5 \%$ & & & & & \\
\hline
\end{tabular}




\begin{tabular}{|c|c|c|c|c|c|c|}
\hline Concentration & $21 \%$ & & & & & \\
\hline Temperature & $80^{\circ} \mathrm{F}$ & & & & & \\
\hline Feed rate & $\begin{array}{c}15 \\
\mathrm{~mm} / \mathrm{s}\end{array}$ & & & & & \\
\hline Needle Gauge & 25 & & & & & \\
\hline Dispense Time & $0.02 \mathrm{~s}$ & & & & & \\
\hline \multicolumn{7}{|l|}{ Short to Long } \\
\hline Fiber \# & $\begin{array}{c}\text { Length } \\
(\mathrm{mm})\end{array}$ & $\begin{array}{c}\text { Leading } \\
\text { Diameter } \\
(\mu \mathrm{m})\end{array}$ & $\begin{array}{c}\text { Middle } \\
\text { Diameter } \\
(\mu \mathrm{m})\end{array}$ & $\begin{array}{c}\text { Terminating } \\
\text { Diameter } \\
(\mu \mathrm{m})\end{array}$ & $\begin{array}{c}\text { Average } \\
\text { Diameter } \\
(\mu \mathrm{m})\end{array}$ & $\begin{array}{c}\text { Standard } \\
\text { Deviation } \\
(\mu \mathrm{m})\end{array}$ \\
\hline 1 & 22 & 0 & 0 & 0 & 0 & 0 \\
\hline 2 & 22 & 0 & 0 & 0 & 0 & 0 \\
\hline 3 & 24 & 0 & 0 & 0 & 0 & 0 \\
\hline 4 & 24 & 4.770 & 8.681 & 10.87 & 8.11 & 2.52 \\
\hline 5 & 26 & 0 & 0 & 0 & 0 & 0 \\
\hline 6 & 26 & 0 & 0 & 0 & 0 & 0 \\
\hline 7 & 28 & 0 & 0 & 0 & 0 & 0 \\
\hline 8 & 28 & 0 & 0 & 0 & 0 & 0 \\
\hline 9 & 30 & 0 & 0 & 0 & 0 & 0 \\
\hline 10 & 30 & 0 & 0 & 0 & 0 & 0 \\
\hline 11 & 32 & 0 & 0 & 0 & 0 & 0 \\
\hline 12 & 32 & 0 & 0 & 0 & 0 & 0 \\
\hline 13 & 34 & 0 & 0 & 0 & 0 & 0 \\
\hline 14 & 34 & 0 & 0 & 0 & 0 & 0 \\
\hline 15 & 36 & 0 & 0 & 0 & 0 & 0 \\
\hline 16 & 36 & 0 & 0 & 0 & 0 & 0 \\
\hline 17 & 38 & 0 & 0 & 0 & 0 & 0 \\
\hline 18 & 38 & 0 & 0 & 0 & 0 & 0 \\
\hline 19 & 40 & 0 & 0 & 0 & 0 & 0 \\
\hline 20 & 40 & 0 & 0 & 0 & 0 & 0 \\
\hline Yield & $5 \%$ & & & & & \\
\hline
\end{tabular}




\begin{tabular}{|c|c|c|c|c|c|c|}
\hline Concentration & $20 \%$ & & & & & \\
\hline Temperature & $100^{\circ} \mathrm{F}$ & & & & & \\
\hline Feed rate & $\begin{array}{c}15 \\
\mathrm{~mm} / \mathrm{s}\end{array}$ & & & & & \\
\hline Needle Gauge & 25 & & & & & \\
\hline Dispense Time & $0.02 \mathrm{~s}$ & & & & & \\
\hline \multicolumn{7}{|l|}{ Short to Long } \\
\hline Fiber \# & $\begin{array}{c}\text { Length } \\
(\mathrm{mm})\end{array}$ & $\begin{array}{c}\text { Leading } \\
\text { Diameter } \\
(\mu \mathrm{m})\end{array}$ & $\begin{array}{c}\text { Middle } \\
\text { Diameter } \\
(\mu \mathrm{m})\end{array}$ & $\begin{array}{c}\text { Terminating } \\
\text { Diameter } \\
(\mu \mathrm{m})\end{array}$ & $\begin{array}{c}\text { Average } \\
\text { Diameter } \\
(\mu \mathrm{m})\end{array}$ & $\begin{array}{c}\text { Standard } \\
\text { Deviation } \\
(\mu \mathrm{m})\end{array}$ \\
\hline 1 & 22 & 1.080 & 0.9951 & 6.918 & 3.00 & 2.77 \\
\hline 2 & 22 & 0 & 0 & 0 & 0 & 0 \\
\hline 3 & 24 & 0 & 0 & 0 & 0 & 0 \\
\hline 4 & 24 & 0 & 0 & 0 & 0 & 0 \\
\hline 5 & 26 & 0 & 0 & 0 & 0 & 0 \\
\hline 6 & 26 & 2.059 & 3.346 & 6.779 & 4.06 & 1.99 \\
\hline 7 & 28 & 0 & 0 & 0 & 0 & 0 \\
\hline 8 & 28 & 0 & 0 & 0 & 0 & 0 \\
\hline 9 & 30 & 0 & 0 & 0 & 0 & 0 \\
\hline 10 & 30 & 0 & 0 & 0 & 0 & 0 \\
\hline 11 & 32 & 0 & 0 & 0 & 0 & 0 \\
\hline 12 & 32 & 0 & 0 & 0 & 0 & 0 \\
\hline 13 & 34 & 0 & 0 & 0 & 0 & 0 \\
\hline 14 & 34 & 1.072 & 3.092 & 8.894 & 4.35 & 3.32 \\
\hline 15 & 36 & 0 & 0 & 0 & 0 & 0 \\
\hline 16 & 36 & 0 & 0 & 0 & 0 & 0 \\
\hline 17 & 38 & 0 & 0 & 0 & 0 & 0 \\
\hline 18 & 38 & 0 & 0 & 0 & 0 & 0 \\
\hline 19 & 40 & 0 & 0 & 0 & 0 & 0 \\
\hline 20 & 40 & 0 & 0 & 0 & 0 & 0 \\
\hline Yield & $15 \%$ & & & & & \\
\hline
\end{tabular}




\begin{tabular}{|c|c|c|c|c|c|c|}
\hline Concentration & $20 \%$ & & & & & \\
\hline Temperature & $100^{\circ} \mathrm{F}$ & & & & & \\
\hline Feed rate & $\begin{array}{c}22.5 \\
\mathrm{~mm} / \mathrm{s}\end{array}$ & & & & & \\
\hline Needle Gauge & 25 & & & & & \\
\hline Dispense Time & $0.02 \mathrm{~s}$ & & & & & \\
\hline \multicolumn{7}{|l|}{ Short to Long } \\
\hline Fiber \# & $\begin{array}{c}\text { Length } \\
(\mathrm{mm})\end{array}$ & $\begin{array}{c}\text { Leading } \\
\text { Diameter } \\
(\mu \mathrm{m})\end{array}$ & $\begin{array}{c}\text { Middle } \\
\text { Diameter } \\
(\mu \mathrm{m})\end{array}$ & $\begin{array}{c}\text { Terminating } \\
\text { Diameter } \\
(\mu \mathrm{m})\end{array}$ & $\begin{array}{c}\text { Average } \\
\text { Diameter } \\
(\mu \mathrm{m})\end{array}$ & $\begin{array}{c}\text { Standard } \\
\text { Deviation } \\
(\mu \mathrm{m})\end{array}$ \\
\hline 1 & 22 & 0 & 0 & 0 & 0 & 0 \\
\hline 2 & 22 & 13.2 & 2.893 & 4.635 & 6.91 & 4.50 \\
\hline 3 & 24 & 0 & 0 & 0 & 0 & 0 \\
\hline 4 & 24 & 0 & 0 & 0 & 0 & 0 \\
\hline 5 & 26 & 0 & 0 & 0 & 0 & 0 \\
\hline 6 & 26 & 11.12 & 15.77 & 5.432 & 10.77 & 4.23 \\
\hline 7 & 28 & 7.734 & 5.610 & 7.279 & 6.87 & 0.91 \\
\hline 8 & 28 & 2.862 & 6.345 & 4.229 & 4.48 & 1.43 \\
\hline 9 & 30 & 7.799 & 8.126 & 6.738 & 7.55 & 0.59 \\
\hline 10 & 30 & 0 & 0 & 0 & 0 & 0 \\
\hline 11 & 32 & 7.902 & 9.140 & 5.213 & 7.42 & 1.64 \\
\hline 12 & 32 & 2.415 & 4.219 & 1.041 & 2.56 & 1.30 \\
\hline 13 & 34 & 2.109 & 3.359 & 4.627 & 3.37 & 1.03 \\
\hline 14 & 34 & 2.072 & 3.862 & 3.327 & 3.09 & 0.75 \\
\hline 15 & 36 & 1.939 & 2.639 & 4.025 & 2.87 & 0.87 \\
\hline 16 & 36 & 2.440 & 4.081 & 4.551 & 3.69 & 0.90 \\
\hline 17 & 38 & 1.779 & 9.713 & 0.9229 & 4.14 & 3.96 \\
\hline 18 & 38 & 3.535 & 0.8758 & 4.605 & 3.01 & 1.57 \\
\hline 19 & 40 & 2.068 & 3.048 & 4.853 & 3.32 & 1.15 \\
\hline 20 & 40 & 0 & 0 & 0 & 0 & 0 \\
\hline Yield & $70 \%$ & & & & & \\
\hline
\end{tabular}




\begin{tabular}{|c|c|c|c|c|c|c|}
\hline Concentration & $20 \%$ & & & & & \\
\hline Temperature & $90^{\circ} \mathrm{F}$ & & & & & \\
\hline Feed rate & $\begin{array}{c}15 \\
\mathrm{~mm} / \mathrm{s}\end{array}$ & & & & & \\
\hline Needle Gauge & 25 & & & & & \\
\hline Dispense Time & $0.02 \mathrm{~s}$ & & & & & \\
\hline \multicolumn{7}{|l|}{ Short to Long } \\
\hline Fiber \# & $\begin{array}{c}\text { Length } \\
(\mathrm{mm})\end{array}$ & $\begin{array}{c}\text { Leading } \\
\text { Diameter } \\
(\mu \mathrm{m})\end{array}$ & $\begin{array}{c}\text { Middle } \\
\text { Diameter } \\
(\mu \mathrm{m})\end{array}$ & $\begin{array}{c}\text { Terminating } \\
\text { Diameter } \\
(\mu \mathrm{m})\end{array}$ & $\begin{array}{c}\text { Average } \\
\text { Diameter } \\
(\mu \mathrm{m})\end{array}$ & $\begin{array}{c}\text { Standard } \\
\text { Deviation } \\
(\mu \mathrm{m})\end{array}$ \\
\hline 1 & 22 & 0 & 0 & 0 & 0 & 0 \\
\hline 2 & 22 & 1.705 & 1.218 & 1.214 & 1.38 & 0.23 \\
\hline 3 & 24 & 1.411 & 1.283 & 1.104 & 1.27 & 0.13 \\
\hline 4 & 24 & 1.013 & 1.838 & 2.623 & 1.82 & 0.66 \\
\hline 5 & 26 & 0 & 0 & 0 & 0 & 0 \\
\hline 6 & 26 & 2.248 & 3.088 & 0.4799 & 1.94 & 1.09 \\
\hline 7 & 28 & 1.040 & 3.480 & 3.082 & 2.53 & 1.07 \\
\hline 8 & 28 & 0.6788 & 0.9814 & 3.872 & 1.84 & 1.44 \\
\hline 9 & 30 & 0 & 0 & 0 & 0 & 0 \\
\hline 10 & 30 & 0 & 0 & 0 & 0 & 0 \\
\hline 11 & 32 & 0 & 0 & 0 & 0 & 0 \\
\hline 12 & 32 & 0 & 0 & 0 & 0 & 0 \\
\hline 13 & 34 & 0 & 0 & 0 & 0 & 0 \\
\hline 14 & 34 & 0 & 0 & 0 & 0 & 0 \\
\hline 15 & 36 & 0 & 0 & 0 & 0 & 0 \\
\hline 16 & 36 & 0 & 0 & 0 & 0 & 0 \\
\hline 17 & 38 & 0 & 0 & 0 & 0 & 0 \\
\hline 18 & 38 & 0 & 0 & 0 & 0 & 0 \\
\hline 19 & 40 & 0 & 0 & 0 & 0 & 0 \\
\hline 20 & 40 & 0 & 0 & 0 & 0 & 0 \\
\hline Yield & $30 \%$ & & & & & \\
\hline
\end{tabular}




\begin{tabular}{|c|c|c|c|c|c|c|}
\hline Concentration & $20 \%$ & & & & & \\
\hline Temperature & $90^{\circ} \mathrm{F}$ & & & & & \\
\hline Feed rate & $\begin{array}{c}22.5 \\
\mathrm{~mm} / \mathrm{s}\end{array}$ & & & & & \\
\hline Needle Gauge & 25 & & & & & \\
\hline Dispense Time & $0.02 \mathrm{~s}$ & & & & & \\
\hline \multicolumn{7}{|l|}{ Short to Long } \\
\hline Fiber \# & $\begin{array}{c}\text { Length } \\
(\mathrm{mm})\end{array}$ & $\begin{array}{c}\text { Leading } \\
\text { Diameter } \\
(\mu \mathrm{m})\end{array}$ & $\begin{array}{c}\text { Middle } \\
\text { Diameter } \\
(\mu \mathrm{m})\end{array}$ & $\begin{array}{c}\text { Terminating } \\
\text { Diameter } \\
(\mu \mathrm{m})\end{array}$ & $\begin{array}{c}\text { Average } \\
\text { Diameter } \\
(\mu \mathrm{m})\end{array}$ & $\begin{array}{c}\text { Standard } \\
\text { Deviation } \\
(\mu \mathrm{m})\end{array}$ \\
\hline 1 & 22 & 5.284 & 3.150 & 4.004 & 4.15 & 0.88 \\
\hline 2 & 22 & 0 & 0 & 0 & 0 & 0 \\
\hline 3 & 24 & 0 & 0 & 0 & 0 & 0 \\
\hline 4 & 24 & 0 & 0 & 0 & 0 & 0 \\
\hline 5 & 26 & 0 & 0 & 0 & 0 & 0 \\
\hline 6 & 26 & 1.975 & 1.499 & 4.563 & 2.68 & 1.35 \\
\hline 7 & 28 & 0 & 0 & 0 & 0 & 0 \\
\hline 8 & 28 & 0 & 0 & 0 & 0 & 0 \\
\hline 9 & 30 & 0 & 0 & 0 & 0 & 0 \\
\hline 10 & 30 & 0 & 0 & 0 & 0 & 0 \\
\hline 11 & 32 & 0 & 0 & 0 & 0 & 0 \\
\hline 12 & 32 & 0 & 0 & 0 & 0 & 0 \\
\hline 13 & 34 & 0 & 0 & 0 & 0 & 0 \\
\hline 14 & 34 & 0 & 0 & 0 & 0 & 0 \\
\hline 15 & 36 & 1.960 & 4.854 & 1.343 & 2.72 & 1.53 \\
\hline 16 & 36 & 0 & 0 & 0 & 0 & 0 \\
\hline 17 & 38 & 1.680 & 4.328 & 3.476 & 3.16 & 1.10 \\
\hline 18 & 38 & 0 & 0 & 0 & 0 & 0 \\
\hline 19 & 40 & 0 & 0 & 0 & 0 & 0 \\
\hline 20 & 40 & 0 & 0 & 0 & 0 & 0 \\
\hline Yield & $20 \%$ & & & & & \\
\hline
\end{tabular}




\begin{tabular}{|c|c|c|c|c|c|c|}
\hline Concentration & $20 \%$ & & & & & \\
\hline Temperature & $80^{\circ} \mathrm{F}$ & & & & & \\
\hline Feed rate & $\begin{array}{c}22.5 \\
\mathrm{~mm} / \mathrm{s}\end{array}$ & & & & & \\
\hline Needle Gauge & 25 & & & & & \\
\hline Dispense Time & $0.02 \mathrm{~s}$ & & & & & \\
\hline \multicolumn{7}{|l|}{ Short to Long } \\
\hline Fiber \# & $\begin{array}{c}\text { Length } \\
(\mathrm{mm})\end{array}$ & $\begin{array}{c}\text { Leading } \\
\text { Diameter } \\
(\mu \mathrm{m})\end{array}$ & $\begin{array}{c}\text { Middle } \\
\text { Diameter } \\
(\mu \mathrm{m})\end{array}$ & $\begin{array}{c}\text { Terminating } \\
\text { Diameter } \\
(\mu \mathrm{m})\end{array}$ & $\begin{array}{c}\text { Average } \\
\text { Diameter } \\
(\mu \mathrm{m})\end{array}$ & $\begin{array}{c}\text { Standard } \\
\text { Deviation } \\
(\mu \mathrm{m})\end{array}$ \\
\hline 1 & 22 & 0 & 0 & 0 & 0 & 0 \\
\hline 2 & 22 & 0 & 0 & 0 & 0 & 0 \\
\hline 3 & 24 & 0 & 0 & 0 & 0 & 0 \\
\hline 4 & 24 & 0 & 0 & 0 & 0 & 0 \\
\hline 5 & 26 & 0 & 0 & 0 & 0 & 0 \\
\hline 6 & 26 & 0 & 0 & 0 & 0 & 0 \\
\hline 7 & 28 & 0 & 0 & 0 & 0 & 0 \\
\hline 8 & 28 & 0 & 0 & 0 & 0 & 0 \\
\hline 9 & 30 & 0 & 0 & 0 & 0 & 0 \\
\hline 10 & 30 & 0 & 0 & 0 & 0 & 0 \\
\hline 11 & 32 & 0 & 0 & 0 & 0 & 0 \\
\hline 12 & 32 & 0 & 0 & 0 & 0 & 0 \\
\hline 13 & 34 & 0 & 0 & 0 & 0 & 0 \\
\hline 14 & 34 & 0 & 0 & 0 & 0 & 0 \\
\hline 15 & 36 & 2.924 & 4.458 & 6.372 & 4.58 & 1.41 \\
\hline 16 & 36 & 0 & 0 & 0 & 0 & 0 \\
\hline 17 & 38 & 0 & 0 & 0 & 0 & 0 \\
\hline 18 & 38 & 0 & 0 & 0 & 0 & 0 \\
\hline 19 & 40 & 0 & 0 & 0 & 0 & 0 \\
\hline 20 & 40 & 0 & 0 & 0 & 0 & 0 \\
\hline Yield & $5 \%$ & & & & & \\
\hline
\end{tabular}




\begin{tabular}{|c|c|c|c|c|c|c|}
\hline Concentration & $20 \%$ & & & & & \\
\hline Temperature & $80^{\circ} \mathrm{F}$ & & & & & \\
\hline Feed rate & $\begin{array}{c}15 \\
\mathrm{~mm} / \mathrm{s}\end{array}$ & & & & & \\
\hline Needle Gauge & 25 & & & & & \\
\hline Dispense Time & $0.02 \mathrm{~s}$ & & & & & \\
\hline \multicolumn{7}{|l|}{ Short to Long } \\
\hline Fiber \# & $\begin{array}{c}\text { Length } \\
(\mathrm{mm})\end{array}$ & $\begin{array}{c}\text { Leading } \\
\text { Diameter } \\
(\mu \mathrm{m})\end{array}$ & $\begin{array}{c}\text { Middle } \\
\text { Diameter } \\
(\mu \mathrm{m})\end{array}$ & $\begin{array}{c}\text { Terminating } \\
\text { Diameter } \\
(\mu \mathrm{m})\end{array}$ & $\begin{array}{c}\text { Average } \\
\text { Diameter } \\
(\mu \mathrm{m})\end{array}$ & $\begin{array}{c}\text { Standard } \\
\text { Deviation } \\
(\mu \mathrm{m})\end{array}$ \\
\hline 1 & 22 & 5.108 & 7.445 & 10.06 & 7.54 & 2.02 \\
\hline 2 & 22 & 0 & 0 & 0 & 0 & 0 \\
\hline 3 & 24 & 0 & 0 & 0 & 0 & 0 \\
\hline 4 & 24 & 0 & 0 & 0 & 0 & 0 \\
\hline 5 & 26 & 0 & 0 & 0 & 0 & 0 \\
\hline 6 & 26 & 0 & 0 & 0 & 0 & 0 \\
\hline 7 & 28 & 0 & 0 & 0 & 0 & 0 \\
\hline 8 & 28 & 0 & 0 & 0 & 0 & 0 \\
\hline 9 & 30 & 0 & 0 & 0 & 0 & 0 \\
\hline 10 & 30 & 0 & 0 & 0 & 0 & 0 \\
\hline 11 & 32 & 0 & 0 & 0 & 0 & 0 \\
\hline 12 & 32 & 0 & 0 & 0 & 0 & 0 \\
\hline 13 & 34 & 0 & 0 & 0 & 0 & 0 \\
\hline 14 & 34 & 0 & 0 & 0 & 0 & 0 \\
\hline 15 & 36 & 0 & 0 & 0 & 0 & 0 \\
\hline 16 & 36 & 0 & 0 & 0 & 0 & 0 \\
\hline 17 & 38 & 0 & 0 & 0 & 0 & 0 \\
\hline 18 & 38 & 0 & 0 & 0 & 0 & 0 \\
\hline 19 & 40 & 0 & 0 & 0 & 0 & 0 \\
\hline 20 & 40 & 0 & 0 & 0 & 0 & 0 \\
\hline Yield & $5 \%$ & & & & & \\
\hline
\end{tabular}




\begin{tabular}{|c|c|c|c|c|c|c|}
\hline Concentration & $19 \%$ & & & & & \\
\hline Temperature & $100^{\circ} \mathrm{F}$ & & & & & \\
\hline Feed rate & $\begin{array}{c}22.5 \\
\mathrm{~mm} / \mathrm{s}\end{array}$ & & & & & \\
\hline Needle Gauge & 25 & & & & & \\
\hline Dispense Time & $0.02 \mathrm{~s}$ & & & & & \\
\hline \multicolumn{7}{|l|}{ Short to Long } \\
\hline Fiber \# & $\begin{array}{c}\text { Length } \\
(\mathrm{mm})\end{array}$ & $\begin{array}{c}\text { Leading } \\
\text { Diameter } \\
(\mu \mathrm{m})\end{array}$ & $\begin{array}{c}\text { Middle } \\
\text { Diameter } \\
(\mu \mathrm{m})\end{array}$ & $\begin{array}{c}\text { Terminating } \\
\text { Diameter } \\
(\mu \mathrm{m})\end{array}$ & $\begin{array}{c}\text { Average } \\
\text { Diameter } \\
(\mu \mathrm{m})\end{array}$ & $\begin{array}{c}\text { Standard } \\
\text { Deviation } \\
(\mu \mathrm{m})\end{array}$ \\
\hline 1 & 22 & 0 & 0 & 0 & 0 & 0 \\
\hline 2 & 22 & 4.886 & 5.025 & 5.186 & 5.03 & 0.12 \\
\hline 3 & 24 & 0 & 0 & 0 & 0 & 0 \\
\hline 4 & 24 & 0 & 0 & 0 & 0 & 0 \\
\hline 5 & 26 & 0 & 0 & 0 & 0 & 0 \\
\hline 6 & 26 & 0 & 0 & 0 & 0 & 0 \\
\hline 7 & 28 & 0 & 0 & 0 & 0 & 0 \\
\hline 8 & 28 & 0 & 0 & 0 & 0 & 0 \\
\hline 9 & 30 & 0.6745 & 0.6870 & 5.848 & 2.40 & 2.44 \\
\hline 10 & 30 & 0.565 & 1.680 & 2.886 & 1.71 & 0.95 \\
\hline 11 & 32 & 0.5978 & 3.046 & 5.473 & 3.04 & 1.99 \\
\hline 12 & 32 & 0 & 0 & 0 & 0 & 0 \\
\hline 13 & 34 & 2.684 & 1.313 & 7.024 & 3.67 & 2.43 \\
\hline 14 & 34 & 0 & 0 & 0 & 0 & 0 \\
\hline 15 & 36 & 0 & 0 & 0 & 0 & 0 \\
\hline 16 & 36 & 0 & 0 & 0 & 0 & 0 \\
\hline 17 & 38 & 0.8534 & 1.675 & 4.624 & 2.38 & 1.62 \\
\hline 18 & 38 & 0 & 0 & 0 & 0 & 0 \\
\hline 19 & 40 & 0 & 0 & 0 & 0 & 0 \\
\hline 20 & 40 & 0 & 0 & 0 & 0 & 0 \\
\hline Yield & $30 \%$ & & & & & \\
\hline
\end{tabular}




\begin{tabular}{|c|c|c|c|c|c|c|}
\hline Concentration & $19 \%$ & & & & & \\
\hline Temperature & $100^{\circ} \mathrm{F}$ & & & & & \\
\hline Feed rate & $\begin{array}{c}22.5 \\
\mathrm{~mm} / \mathrm{s}\end{array}$ & & & & & \\
\hline Needle Gauge & 25 & & & & & \\
\hline Dispense Time & $0.02 \mathrm{~s}$ & & & & & \\
\hline \multicolumn{7}{|l|}{ Short to Long } \\
\hline Fiber \# & $\begin{array}{c}\text { Length } \\
(\mathrm{mm})\end{array}$ & $\begin{array}{c}\text { Leading } \\
\text { Diameter } \\
(\mu \mathrm{m})\end{array}$ & $\begin{array}{c}\text { Middle } \\
\text { Diameter } \\
(\mu \mathrm{m})\end{array}$ & $\begin{array}{c}\text { Terminating } \\
\text { Diameter } \\
(\mu \mathrm{m})\end{array}$ & $\begin{array}{c}\text { Average } \\
\text { Diameter } \\
(\mu \mathrm{m})\end{array}$ & $\begin{array}{c}\text { Standard } \\
\text { Deviation } \\
(\mu \mathrm{m})\end{array}$ \\
\hline 1 & 22 & 0.4684 & 0.3812 & 1.327 & 0.73 & 0.43 \\
\hline 2 & 22 & 1.025 & 2.471 & 1.447 & 1.65 & 0.61 \\
\hline 3 & 24 & 0.829 & 0.969 & 1.5 & 1.10 & 0.29 \\
\hline 4 & 24 & 0 & 0 & 0 & 0 & 0 \\
\hline 5 & 26 & 0 & 0 & 0 & 0 & 0 \\
\hline 6 & 26 & 0 & 0 & 0 & 0 & 0 \\
\hline 7 & 28 & 0.9804 & 1.712 & 0.957 & 1.22 & 0.35 \\
\hline 8 & 28 & 0 & 0 & 0 & 0 & 0 \\
\hline 9 & 30 & 0.7105 & 0.2094 & 1.646 & 0.86 & 0.60 \\
\hline 10 & 30 & 0 & 0 & 0 & 0 & 0 \\
\hline 11 & 32 & 0.5746 & 0.2701 & 1.057 & 0.63 & 0.32 \\
\hline 12 & 32 & 0 & 0 & 0 & 0 & 0 \\
\hline 13 & 34 & 0 & 0 & 0 & 0 & 0 \\
\hline 14 & 34 & 0 & 0 & 0 & 0 & 0 \\
\hline 15 & 36 & 0 & 0 & 0 & 0 & 0 \\
\hline 16 & 36 & 0 & 0 & 0 & 0 & 0 \\
\hline 17 & 38 & 0 & 0 & 0 & 0 & 0 \\
\hline 18 & 38 & 0 & 0 & 0 & 0 & 0 \\
\hline 19 & 40 & 0 & 0 & 0 & 0 & 0 \\
\hline 20 & 40 & 0 & 0 & 0 & 0 & 0 \\
\hline Yield & $30 \%$ & & & & & \\
\hline
\end{tabular}




\begin{tabular}{|c|c|c|c|c|c|c|}
\hline Concentration & $19 \%$ & & & & & \\
\hline Temperature & $90^{\circ} \mathrm{F}$ & & & & & \\
\hline Feed rate & $\begin{array}{c}22.5 \\
\mathrm{~mm} / \mathrm{s}\end{array}$ & & & & & \\
\hline Needle Gauge & 25 & & & & & \\
\hline Dispense Time & $0.02 \mathrm{~s}$ & & & & & \\
\hline \multicolumn{7}{|l|}{ Short to Long } \\
\hline Fiber \# & $\begin{array}{c}\text { Length } \\
(\mathrm{mm})\end{array}$ & $\begin{array}{c}\text { Leading } \\
\text { Diameter } \\
(\mu \mathrm{m})\end{array}$ & $\begin{array}{c}\text { Middle } \\
\text { Diameter } \\
(\mu \mathrm{m})\end{array}$ & $\begin{array}{c}\text { Terminating } \\
\text { Diameter } \\
(\mu \mathrm{m})\end{array}$ & $\begin{array}{c}\text { Average } \\
\text { Diameter } \\
(\mu \mathrm{m})\end{array}$ & $\begin{array}{c}\text { Standard } \\
\text { Deviation } \\
(\mu \mathrm{m})\end{array}$ \\
\hline 1 & 22 & 0 & 0 & 0 & 0 & 0 \\
\hline 2 & 22 & 1.156 & 3.335 & 5.345 & 3.28 & 1.71 \\
\hline 3 & 24 & 1.312 & 0.8637 & 1.438 & 1.20 & 0.25 \\
\hline 4 & 24 & 0.5857 & 4.032 & 4.147 & 2.92 & 1.65 \\
\hline 5 & 26 & 0.9973 & 2.876 & 2.417 & 2.10 & 0.80 \\
\hline 6 & 26 & 0.4719 & 2.219 & 3.001 & 1.90 & 1.06 \\
\hline 7 & 28 & 0.5296 & 0.5039 & 2.633 & 1.22 & 1.00 \\
\hline 8 & 28 & 0 & 0 & 0 & 0 & 0 \\
\hline 9 & 30 & 0 & 0 & 0 & 0 & 0 \\
\hline 10 & 30 & 0 & 0 & 0 & 0 & 0 \\
\hline 11 & 32 & 0 & 0 & 0 & 0 & 0 \\
\hline 12 & 32 & 0 & 0 & 0 & 0 & 0 \\
\hline 13 & 34 & 0 & 0 & 0 & 0 & 0 \\
\hline 14 & 34 & 0 & 0 & 0 & 0 & 0 \\
\hline 15 & 36 & 0 & 0 & 0 & 0 & 0 \\
\hline 16 & 36 & 0 & 0 & 0 & 0 & 0 \\
\hline 17 & 38 & 0 & 0 & 0 & 0 & 0 \\
\hline 18 & 38 & 0 & 0 & 0 & 0 & 0 \\
\hline 19 & 40 & 0 & 0 & 0 & 0 & 0 \\
\hline 20 & 40 & 0 & 0 & 0 & 0 & 0 \\
\hline Yield & $30 \%$ & & & & & \\
\hline
\end{tabular}




\begin{tabular}{|c|c|c|c|c|c|c|}
\hline Concentration & $19 \%$ & & & & & \\
\hline Temperature & $90^{\circ} \mathrm{F}$ & & & & & \\
\hline Feed rate & $\begin{array}{c}22.5 \\
\mathrm{~mm} / \mathrm{s}\end{array}$ & & & & & \\
\hline Needle Gauge & 25 & & & & & \\
\hline Dispense Time & $0.02 \mathrm{~s}$ & & & & & \\
\hline \multicolumn{7}{|l|}{ Short to Long } \\
\hline Fiber \# & $\begin{array}{c}\text { Length } \\
(\mathrm{mm})\end{array}$ & $\begin{array}{c}\text { Leading } \\
\text { Diameter } \\
(\mu \mathrm{m})\end{array}$ & $\begin{array}{c}\text { Middle } \\
\text { Diameter } \\
(\mu \mathrm{m})\end{array}$ & $\begin{array}{c}\text { Terminating } \\
\text { Diameter } \\
(\mu \mathrm{m})\end{array}$ & $\begin{array}{c}\text { Average } \\
\text { Diameter } \\
(\mu \mathrm{m})\end{array}$ & $\begin{array}{c}\text { Standard } \\
\text { Deviation } \\
(\mu \mathrm{m})\end{array}$ \\
\hline 1 & 22 & 0 & 0 & 0 & 0 & 0 \\
\hline 2 & 22 & 0 & 0 & 0 & 0 & 0 \\
\hline 3 & 24 & 2.338 & 5.561 & 3.328 & 3.74 & 1.35 \\
\hline 4 & 24 & 2.413 & 3.486 & 4.428 & 3.44 & 0.82 \\
\hline 5 & 26 & 0 & 0 & 0 & 0 & 0 \\
\hline 6 & 26 & 0 & 0 & 0 & 0 & 0 \\
\hline 7 & 28 & 0 & 0 & 0 & 0 & 0 \\
\hline 8 & 28 & 0 & 0 & 0 & 0 & 0 \\
\hline 9 & 30 & 0 & 0 & 0 & 0 & 0 \\
\hline 10 & 30 & 0 & 0 & 0 & 0 & 0 \\
\hline 11 & 32 & 0 & 0 & 0 & 0 & 0 \\
\hline 12 & 32 & 0 & 0 & 0 & 0 & 0 \\
\hline 13 & 34 & 0 & 0 & 0 & 0 & 0 \\
\hline 14 & 34 & 0 & 0 & 0 & 0 & 0 \\
\hline 15 & 36 & 0 & 0 & 0 & 0 & 0 \\
\hline 16 & 36 & 0 & 0 & 0 & 0 & 0 \\
\hline 17 & 38 & 0 & 0 & 0 & 0 & 0 \\
\hline 18 & 38 & 0 & 0 & 0 & 0 & 0 \\
\hline 19 & 40 & 0 & 0 & 0 & 0 & 0 \\
\hline 20 & 40 & 0 & 0 & 0 & 0 & 0 \\
\hline Yield & $10 \%$ & & & & & \\
\hline
\end{tabular}




\begin{tabular}{|c|c|c|c|c|c|c|}
\hline Concentration & $19 \%$ & & & & & \\
\hline Temperature & $80^{\circ} \mathrm{F}$ & & & & & \\
\hline Feed rate & $\begin{array}{c}22.5 \\
\mathrm{~mm} / \mathrm{s}\end{array}$ & & & & & \\
\hline Needle Gauge & 25 & & & & & \\
\hline Dispense Time & $0.02 \mathrm{~s}$ & & & & & \\
\hline \multicolumn{7}{|l|}{ Short to Long } \\
\hline Fiber \# & $\begin{array}{c}\text { Length } \\
(\mathrm{mm})\end{array}$ & $\begin{array}{c}\text { Leading } \\
\text { Diameter } \\
(\mu \mathrm{m})\end{array}$ & $\begin{array}{c}\text { Middle } \\
\text { Diameter } \\
(\mu \mathrm{m})\end{array}$ & $\begin{array}{c}\text { Terminating } \\
\text { Diameter } \\
(\mu \mathrm{m})\end{array}$ & $\begin{array}{c}\text { Average } \\
\text { Diameter } \\
(\mu \mathrm{m})\end{array}$ & $\begin{array}{c}\text { Standard } \\
\text { Deviation } \\
(\mu \mathrm{m})\end{array}$ \\
\hline 1 & 22 & 4.607 & 3.939 & 1.310 & 3.29 & 1.42 \\
\hline 2 & 22 & 0.6925 & 1.132 & 2.281 & 1.37 & 0.67 \\
\hline 3 & 24 & 1.695 & 0.7924 & 0.6715 & 1.05 & 0.46 \\
\hline 4 & 24 & 0.645 & 1.618 & 1.432 & 1.23 & 0.42 \\
\hline 5 & 26 & 0.7468 & 3.571 & 3.812 & 2.71 & 1.39 \\
\hline 6 & 26 & 0 & 0 & 0 & 0 & 0 \\
\hline 7 & 28 & 0 & 0 & 0 & 0 & 0 \\
\hline 8 & 28 & 0 & 0 & 0 & 0 & 0 \\
\hline 9 & 30 & 0 & 0 & 0 & 0 & 0 \\
\hline 10 & 30 & 1.826 & 2.838 & 3.968 & 2.88 & 0.87 \\
\hline 11 & 32 & 0 & 0 & 0 & 0 & 0 \\
\hline 12 & 32 & 1.018 & 1.047 & 2.710 & 1.59 & 0.79 \\
\hline 13 & 34 & 0.9646 & 2.264 & 2.670 & 1.97 & 0.73 \\
\hline 14 & 34 & 0 & 0 & 0 & 0 & 0 \\
\hline 15 & 36 & 0 & 0 & 0 & 0 & 0 \\
\hline 16 & 36 & 0 & 0 & 0 & 0 & 0 \\
\hline 17 & 38 & 0 & 0 & 0 & 0 & 0 \\
\hline 18 & 38 & 0 & 0 & 0 & 0 & 0 \\
\hline 19 & 40 & 0 & 0 & 0 & 0 & 0 \\
\hline 20 & 40 & 0 & 0 & 0 & 0 & 0 \\
\hline Yield & $40 \%$ & & & & & \\
\hline
\end{tabular}




\begin{tabular}{|c|c|c|c|c|c|c|}
\hline Concentration & $19 \%$ & & & & & \\
\hline Temperature & $80^{\circ} \mathrm{F}$ & & & & & \\
\hline Feed rate & $\begin{array}{c}22.5 \\
\mathrm{~mm} / \mathrm{s}\end{array}$ & & & & & \\
\hline Needle Gauge & 25 & & & & & \\
\hline Dispense Time & $0.02 \mathrm{~s}$ & & & & & \\
\hline \multicolumn{7}{|l|}{ Short to Long } \\
\hline Fiber \# & $\begin{array}{c}\text { Length } \\
(\mathrm{mm})\end{array}$ & $\begin{array}{c}\text { Leading } \\
\text { Diameter } \\
(\mu \mathrm{m})\end{array}$ & $\begin{array}{c}\text { Middle } \\
\text { Diameter } \\
(\mu \mathrm{m})\end{array}$ & $\begin{array}{c}\text { Terminating } \\
\text { Diameter } \\
(\mu \mathrm{m})\end{array}$ & $\begin{array}{c}\text { Average } \\
\text { Diameter } \\
(\mu \mathrm{m})\end{array}$ & $\begin{array}{c}\text { Standard } \\
\text { Deviation } \\
(\mu \mathrm{m})\end{array}$ \\
\hline 1 & 22 & 1.264 & 1.813 & 1.097 & 1.39 & 0.31 \\
\hline 2 & 22 & 2.019 & 1.169 & 1.463 & 1.55 & 0.35 \\
\hline 3 & 24 & 0.9796 & 0.6638 & 0.5786 & 0.74 & 0.17 \\
\hline 4 & 24 & 0.747 & 1.875 & 3.122 & 1.91 & 0.97 \\
\hline 5 & 26 & 0.7588 & 2.102 & 5.427 & 2.76 & 1.96 \\
\hline 6 & 26 & 0 & 0 & 0 & 0 & 0 \\
\hline 7 & 28 & 0 & 0 & 0 & 0 & 0 \\
\hline 8 & 28 & 0 & 0 & 0 & 0 & 0 \\
\hline 9 & 30 & 0 & 0 & 0 & 0 & 0 \\
\hline 10 & 30 & 0 & 0 & 0 & 0 & 0 \\
\hline 11 & 32 & 0 & 0 & 0 & 0 & 0 \\
\hline 12 & 32 & 0 & 0 & 0 & 0 & 0 \\
\hline 13 & 34 & 0 & 0 & 0 & 0 & 0 \\
\hline 14 & 34 & 0 & 0 & 0 & 0 & 0 \\
\hline 15 & 36 & 0.7512 & 0.5069 & 0.7982 & 0.69 & 0.13 \\
\hline 16 & 36 & 2.415 & 2.669 & 0.6248 & 1.90 & 0.91 \\
\hline 17 & 38 & 0 & 0 & 0 & 0 & 0 \\
\hline 18 & 38 & 0.473 & 1.523 & 2.949 & 1.65 & 1.01 \\
\hline 19 & 40 & 0 & 0 & 0 & 0 & 0 \\
\hline 20 & 40 & 0 & 0 & 0 & 0 & 0 \\
\hline Yield & $40 \%$ & & & & & \\
\hline
\end{tabular}




\begin{tabular}{|c|c|c|c|c|c|c|}
\hline Concentration & $19 \%$ & & & & & \\
\hline Temperature & $70^{\circ} \mathrm{F}$ & & & & & \\
\hline Feed rate & $\begin{array}{c}22.5 \\
\mathrm{~mm} / \mathrm{s}\end{array}$ & & & & & \\
\hline Needle Gauge & 25 & & & & & \\
\hline Dispense Time & $0.02 \mathrm{~s}$ & & & & & \\
\hline \multicolumn{7}{|l|}{ Short to Long } \\
\hline Fiber \# & $\begin{array}{c}\text { Length } \\
(\mathrm{mm})\end{array}$ & $\begin{array}{c}\text { Leading } \\
\text { Diameter } \\
(\mu \mathrm{m})\end{array}$ & $\begin{array}{c}\text { Middle } \\
\text { Diameter } \\
(\mu \mathrm{m})\end{array}$ & $\begin{array}{c}\text { Terminating } \\
\text { Diameter } \\
(\mu \mathrm{m})\end{array}$ & $\begin{array}{c}\text { Average } \\
\text { Diameter } \\
(\mu \mathrm{m})\end{array}$ & $\begin{array}{c}\text { Standard } \\
\text { Deviation } \\
(\mu \mathrm{m})\end{array}$ \\
\hline 1 & 22 & 0.4671 & 0.3418 & 0.2913 & 0.37 & 0.07 \\
\hline 2 & 22 & 0.2732 & 0.1421 & 0.4814 & 0.30 & 0.14 \\
\hline 3 & 24 & 0.3817 & 0.2823 & 0.1398 & 0.27 & 0.10 \\
\hline 4 & 24 & 0.6898 & 0.3646 & 0.4316 & 0.50 & 0.14 \\
\hline 5 & 26 & 0.7328 & 0.3395 & 0.4495 & 0.51 & 0.17 \\
\hline 6 & 26 & 0.4627 & 0.3774 & 0.4035 & 0.41 & 0.04 \\
\hline 7 & 28 & 0.1533 & 0.5539 & 0.6408 & 0.45 & 0.21 \\
\hline 8 & 28 & 0.2064 & 0.4978 & 0.5045 & 0.40 & 0.14 \\
\hline 9 & 30 & 0 & 0 & 0 & 0 & 0 \\
\hline 10 & 30 & 0 & 0 & 0 & 0 & 0 \\
\hline 11 & 32 & 0 & 0 & 0 & 0 & 0 \\
\hline 12 & 32 & 0 & 0 & 0 & 0 & 0 \\
\hline 13 & 34 & 0 & 0 & 0 & 0 & 0 \\
\hline 14 & 34 & 0 & 0 & 0 & 0 & 0 \\
\hline 15 & 36 & 0 & 0 & 0 & 0 & 0 \\
\hline 16 & 36 & 0 & 0 & 0 & 0 & 0 \\
\hline 17 & 38 & 0 & 0 & 0 & 0 & 0 \\
\hline 18 & 38 & 0 & 0 & 0 & 0 & 0 \\
\hline 19 & 40 & 0 & 0 & 0 & 0 & 0 \\
\hline 20 & 40 & 0 & 0 & 0 & 0 & 0 \\
\hline Yield & $40 \%$ & & & & & \\
\hline
\end{tabular}




\begin{tabular}{|c|c|c|c|c|c|c|}
\hline Concentration & $19 \%$ & & & & & \\
\hline Temperature & $70^{\circ} \mathrm{F}$ & & & & & \\
\hline Feed rate & $\begin{array}{c}22.5 \\
\mathrm{~mm} / \mathrm{s}\end{array}$ & & & & & \\
\hline Needle Gauge & 25 & & & & & \\
\hline Dispense Time & $0.02 \mathrm{~s}$ & & & & & \\
\hline \multicolumn{7}{|l|}{ Short to Long } \\
\hline Fiber \# & $\begin{array}{c}\text { Length } \\
(\mathrm{mm})\end{array}$ & $\begin{array}{c}\text { Leading } \\
\text { Diameter } \\
(\mu \mathrm{m})\end{array}$ & $\begin{array}{c}\text { Middle } \\
\text { Diameter } \\
(\mu \mathrm{m})\end{array}$ & $\begin{array}{c}\text { Terminating } \\
\text { Diameter } \\
(\mu \mathrm{m})\end{array}$ & $\begin{array}{c}\text { Average } \\
\text { Diameter } \\
(\mu \mathrm{m})\end{array}$ & $\begin{array}{c}\text { Standard } \\
\text { Deviation } \\
(\mu \mathrm{m})\end{array}$ \\
\hline 1 & 22 & 1.909 & 0.3343 & 0.3405 & 0.86 & 0.74 \\
\hline 2 & 22 & 0.3886 & 0.2448 & 0.1786 & 0.27 & 0.09 \\
\hline 3 & 24 & 0.3601 & 0.2466 & 0.144 & 0.25 & 0.09 \\
\hline 4 & 24 & 0.4614 & 0.2175 & 0.2694 & 0.32 & 0.10 \\
\hline 5 & 26 & 0.4709 & 0.407 & 0.317 & 0.40 & 0.06 \\
\hline 6 & 26 & 0.6705 & 0.446 & 0.3338 & 0.48 & 0.14 \\
\hline 7 & 28 & 0 & 0 & 0 & 0 & 0 \\
\hline 8 & 28 & 0 & 0 & 0 & 0 & 0 \\
\hline 9 & 30 & 0 & 0 & 0 & 0 & 0 \\
\hline 10 & 30 & 0 & 0 & 0 & 0 & 0 \\
\hline 11 & 32 & 0 & 0 & 0 & 0 & 0 \\
\hline 12 & 32 & 0 & 0 & 0 & 0 & 0 \\
\hline 13 & 34 & 0 & 0 & 0 & 0 & 0 \\
\hline 14 & 34 & 0 & 0 & 0 & 0 & 0 \\
\hline 15 & 36 & 0 & 0 & 0 & 0 & 0 \\
\hline 16 & 36 & 0 & 0 & 0 & 0 & 0 \\
\hline 17 & 38 & 0 & 0 & 0 & 0 & 0 \\
\hline 18 & 38 & 0 & 0 & 0 & 0 & 0 \\
\hline 19 & 40 & 0 & 0 & 0 & 0 & 0 \\
\hline 20 & 40 & 0 & 0 & 0 & 0 & 0 \\
\hline Yield & $30 \%$ & & & & & \\
\hline
\end{tabular}




\title{
CURRICULUM VITA
}

\author{
NAME: Hanwen Yuan \\ Speed School of Engineering \\ University of Louisville \\ Louisville, KY 40292 \\ 502-852-0539 \\ hanwen.yuan@1ouisville.edu
}

OBJECTIVE To obtain a micro/nano fabrication process engineer position that utilizes my research and development, design, communication and project management skills.

SUMMARY Ph.D. degree in mechanical engineering and master degree in control theory and engineering with experience in research and design concentrating in:

- 5 years of Micro/Nano fabrication (photolithography, wet/dry/metal etching, replica molding, alignment and bonding, mask design)

- 2 years of Equipment maintenance, parameter monitoring (March RIE, SYS DRIE, 3-axis Robotic system, Viscometer, Micro-Milling Machine)

- 2 years of thin film solar cell design and fab (RCA, oxidation, BOE etching, sputtering, annealing)

- 5 years of Micro/Nano fibers creation (DOE, Minitab)

- 3 years of process simulation (SolidWorks, Matlab, LabVIEW, Multisim)

- 7 years of statistical data analysis (SQL server, Java, Python)

- 5 years of metrology and process analysis tools (SEM, AFM, Raman, Confocal Microscope, Zygo Optical Interferometer, Surface Profilometer, XPS, TGA, Viscometer)

- 2 years of Management software development to improve functionality of time scheduling, client data collection, equipment usage (Java, Python, SSIS, SSRS)

EXPERIENCE The University of Louisville (UofL), KY

2011 to 2017

\section{Graduate Fellowship}

- Designed \& Fabricated drug delivery micro scale spray nozzles

- Designed \& Fabricated high efficient, cheap thin film silicon solar cells

- Created complex and suspended 3-D micro fiber structures

- Characterized and optimized the fiber writing process parameters to create repeatable, controllable, oriented, and suspended sub-micro/nano scale fibers 
- Designed \& Developed nanoscale fluidic devices to create multiple channels of varying sizes embedded in a single fluidic platform

- Machined parts to build high precision alignment and bonding device

- Designed electric circuit to generate high voltage pulse using relay, source meter, waveform function generator and oscilloscope

- Laser trapped cells to desired locations in micro/nano scale 3$\mathrm{D}$ devices, and captured PI dyes travelling through nanochannels

- Designed \& assembled LabVIEW data acquisition system based on NI USB-6009 to analyze multichannel signals

- Designed the layout of devices or parts using SolidWorks

Shenzhen Onetouch Technology Co., Ltd., China, 2009 to 2011 Software Developer \& Data Analysist

- Designed \& Developed GSM Network Optimization application website and analysis tools with SQL, Java, and Python

- Maintained mobile system database, effectively communicated with clients to improve their work efficiency including equipment scheduling, work flow, equipment usage, maintenance log

- Developed software requirements to improve the communication efficiency between clients and team leaders of database design and code design to accelerate the development progress

- Analyzed clients' data and generated professional summary reports to assist clients in optimizing their work flow

The South China University of Technology (SCUT), China

Graduate Research Assistantship

- Designed \& Simulated an adaptive neural network algorithm to control autonomous underwater vehicles' (AUVs) diving motion under complex environment by approximating their dynamics

- Designed \& Simulated an adaptive neural network algorithm to resolve the inherent tradeoffs among ride quality, handling performance, and suspension travel of the active car suspension systems

- Applied deterministic learning and rapid dynamical pattern recognition method to determine the occasion of malfunctions of rotating stall and surge for aeroengine, then to restrain and delay further bad effect

EDUCATION The University of Louisville, KY. G.P.A.: 3.97

2011-2017

Ph.D. in Mechanical Engineering 
The South China University of Technology, China. G.P.A.: 3.25

M.S. in Control Theory and Engineering

Jianghan University (JU), China. Major Ranking: Top 20\%

$2002-2006$

B.S. in Measurement and Control Technology and Instrumentation

COMPUTER

SKILLS

HONORS

PUBLICATIONS
- Modeling/Simulation: Multisim, Labview

- Design: L-edit, Silvaco Athena, SolidWorks, Photoshop, MS office

- Data Analysis: Matlab, MathCAD, Maple, Igor, Minitab, Image J

- Language: C, JAVA, Python, SQL

- Graduate Fellowship, UofL 2013-2017

- University Graduate Fellowship, UofL 2011 - 2013

- First Class Scholarship for Outstanding Students, SCUT

- Hua Wei Scholarship, SCUT $2007-2008$

- Third Class Scholarship, JU 2004-2005

- Merit student (good in study, attitude and health), JU

$2004-2005$

- Hanwen Yuan, Yuelong Liu, Cong Wang, "Nonlinear Adaptive Neural Network Controller Design for Half-Car Active Suspension Systems", 5th International Symposium on Neural Networks, September 2008.

- Hanwen Yuan, Cong Wang, "Adaptive NN Controller Design for an Autonomous Underwater Vehicle", 27th Chinese Control Conference, July 2008.

- Cong Wang, Tao Peng, Tianrui Chen, and Hanwen Yuan, "Identification and rapid detection of rotating stall via deterministic learning", 29th Chinese Control Conference, July 2010 .

- Hanwen Yuan, Scott Cambron, and Robert Keynton, "Prescribed 3-D Direct Writing of Suspended Micron/Submicron Scale Fiber Structures via a Robotic Dispensing System," Journal of Visualized Experiments, p. 52834, 2015.

- Hanwen Yuan, Scott Cambron, Mark Crain, and Robert Keynton, "Fabrication of a Micro/Nanofluidic Platform via 3Axis Robotic Dispensing System," Proceedings of the ASME 2016 International Manufacturing Science and Engineering Conference, July, 2016.

- Hanwen Yuan, Scott Cambron, Mark Crain, and Robert Keynton, "Fabrication of a Micro/Nanofluidic Platform via 3- 
Axis Robotic Dispensing System," Journal of Micro and NanoManufacturing, 4(4), 041005, 2016.

- Hanwen Yuan, Scott Cambron, and Robert Keynton, "Precisely drawing repeatable and controllable sub-micron scale PMMA fibers via robotic dispensing system," to be submitted, 2017.

- Hanwen Yuan, Scott Cambron, and Robert Keynton, "Optimization of PMMA fibers fabrication via robotic dispensing system," to be submitted, 2017. 